\title{
Concussion Among Military Service Academy Members: Identifying Risk Factors, Recovery Trajectories, And The Role Of Mental Health
}

\author{
by \\ Kathryn L. O’Connor \\ A dissertation submitted in partial fulfillment \\ of the requirements for the degree of \\ Doctor of Philosophy \\ (Kinesiology) \\ in The University of Michigan \\ 2018
}

Doctoral Committee:

Associate Professor Steven P. Broglio, Chair

Dr. Darren Campbell, United States Air Force Academy

Professor Xuming He

Professor Thomas W. McAllister, Indiana University

Professor Michael McCrea, Medical College of Wisconsin

Assistant Professor Sean K. Meehan

Dr. Steven J. Svoboda, United States Military Academy 


\title{
Kathryn L. O’Connor
}

\author{
kloconn@umich.edu
}

ORCID iD: 0000-0002-5494-2357

(C) Kathryn L. O’Connor 2018 


\section{ACKNOWLEDGEMENTS}

Dr. McAllister asked me a couple years ago "did you ever think your PhD experience would be anything like this?". Never could I have expected to have such a remarkable, fulfilling, adventurous, and developmental $\mathrm{PhD}$ experience. My experience is due to all the amazing individuals referenced below. However, my thanks cannot be adequately expressed in this brief section.

Steve, thank you for your guidance, support, and the opportunity to join your lab. You taught me to seek out and challenge my weaknesses, pushing me, but never beyond my limits. I have learned more in the past few years than I ever thought possible. Although I do not think I can thank you enough, I can forgive you for offering me up to a lion that one time...

To my dissertation committee, Xuming, Sean, Steve, Darren, Mike, and Tom, it takes a village to raise a PhD student and I had an amazing village behind me. Each of you have provided me with enduring support, unique perspectives, and insight. This dissertation would not have been possible without your help.

Additionally, I would like to thank the research staff at each of the Service Academies. Without their tireless efforts I would not have been able to complete these projects. And to the cadets, a special thank you for volunteering to participate in this study.

In my brief summer on North Campus and attempting to remember high school physics, JT and James, you provided me with a unique and challenging summer internship. I also learned an enumerable amount regarding grantmanship during our early breakfast meetings.

To the NeuroTrauma lab, Doug, Richelle, Andrew, Margaret, Jennylee, and Tessa thank you for your constant support throughout my dissertation. You each have been a sounding board and have made it a joy to come into lab each day. Our failed championship dodgeball run, nerf wars, and whirlyball are just few of many highlights. A special thank you to Ashley, who does all the "behind the scenes" work, but also has become a great friend. Thank you for always listening and advice to "make good choices".

Charlene, thank you for always checking in and helping make my experience at Michigan so wonderful. No matter how busy you got you always made time for us students. I am sure other students, like myself, can't ever thank you enough.

To my friends, both here and afar, thank you for your support and helping me procrastinate when needed. Thank you for always letting me talk science and thank you especially for not appearing to be bored. 
Doug, thank you for your constant support, motivation, understanding, and thoughtfulness. Also, you sometimes have some pretty good jokes. You above all else have made my time at Michigan memorable. Now I am looking forward to making many new and exciting memories together.

To my family, thank you for being my foundation. Mom and Dad, thank you for providing me with every opportunity I could have ever dreamed of and more. But most importantly, thank you for your example. From you, I learned the benefit of hard work, inquisitiveness, motivation, and character. 


\section{TABLE OF CONTENTS}

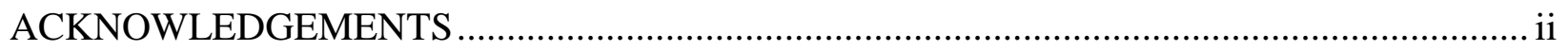

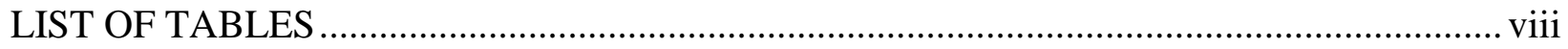

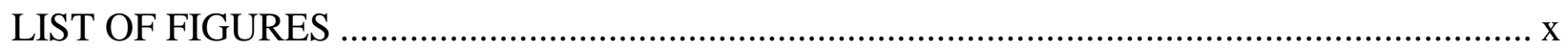

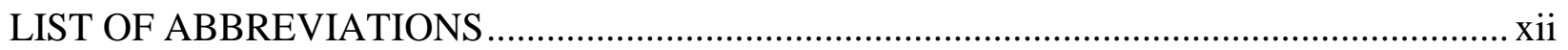

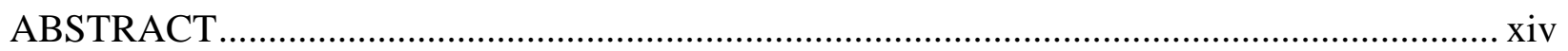

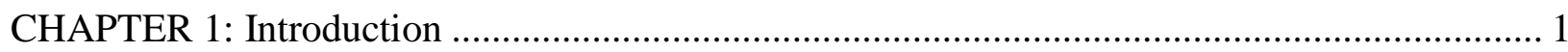

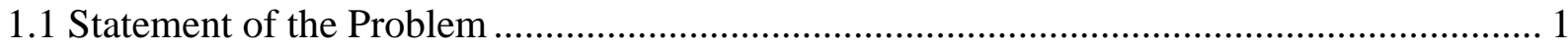

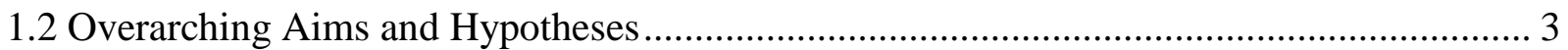

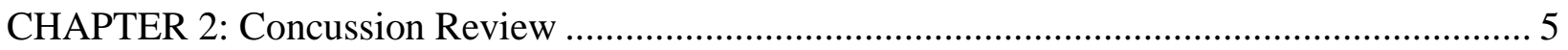

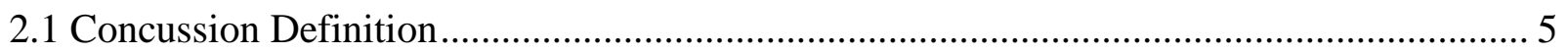

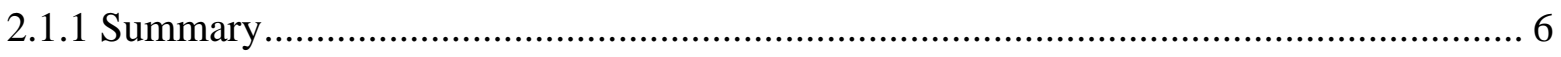

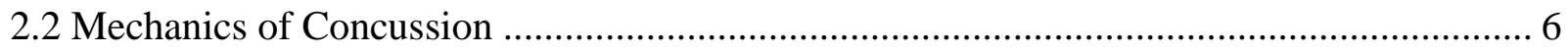

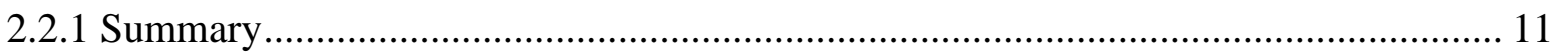

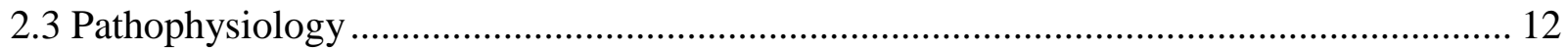

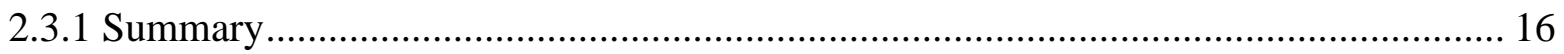

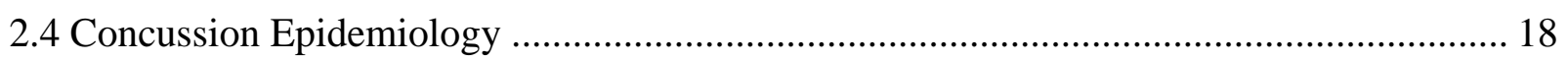

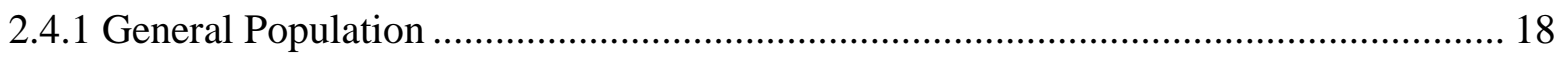

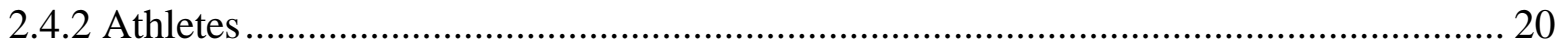

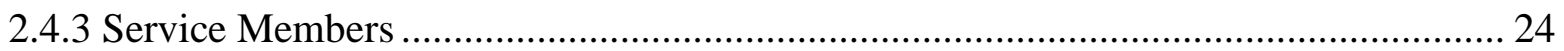

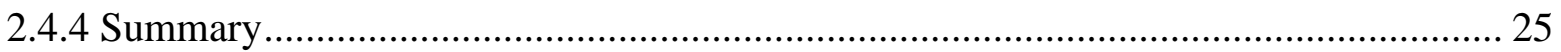

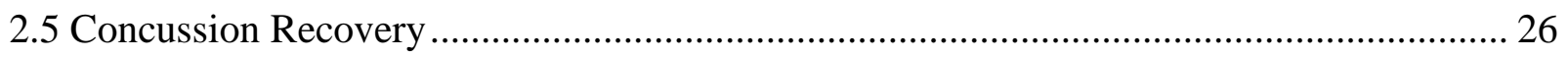

2.5.1 Factors Associated with Concussion Symptom Duration ........................................... 27

2.5.2 Factors Associated with Total Time Loss after Concussion ........................................ 31

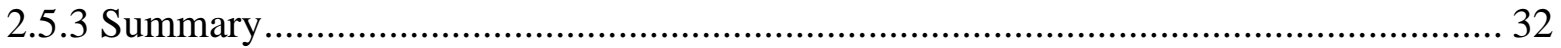

2.6 The Association between Concussion and Mental Health .................................................. 33

2.6.1 Psychological Symptoms Associate with Injury Risk ................................................. 33

2.6.2 Concussion's Impact on Subsequent Mental Health .................................................... 35 


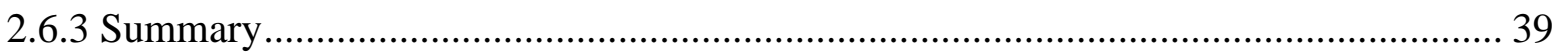

2.7 United States Service Academies and Cadets ……………….............................................. 40

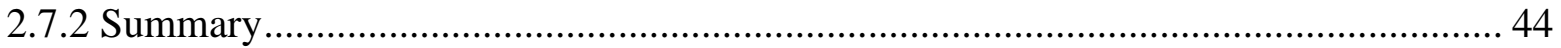

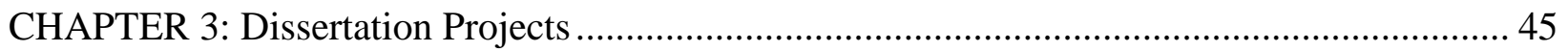

Chapter 3.1 Concussion Assessment, Research and Education Consortium ............................ 45

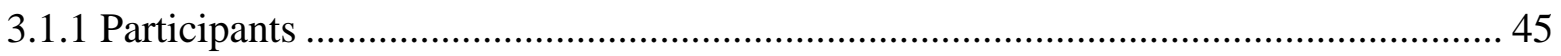

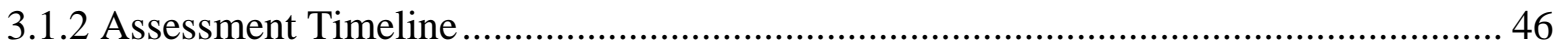

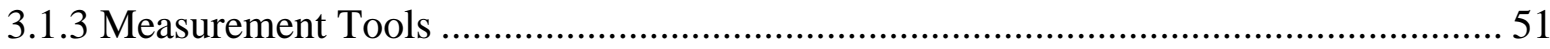

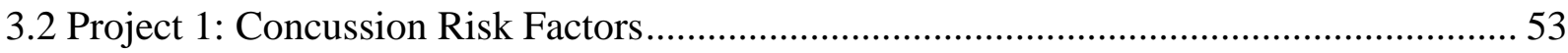

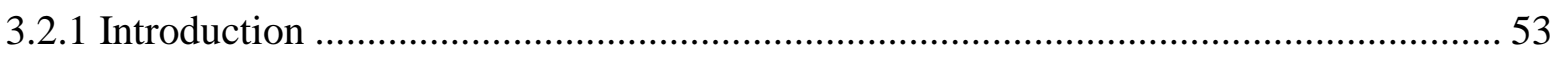

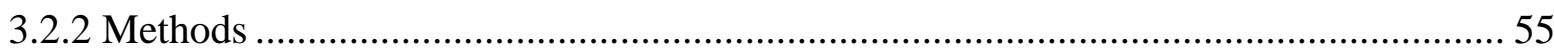

3.2.2.1 Study Design ............................................................................................... 55

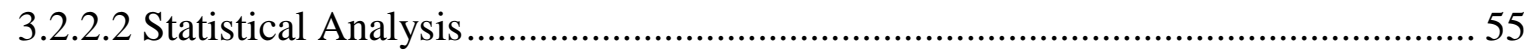

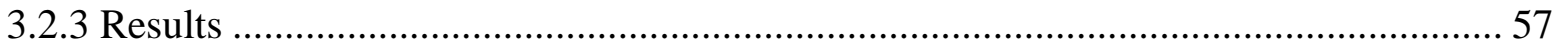

3.2.3.1 Cadet Characteristics ............................................................................... 57

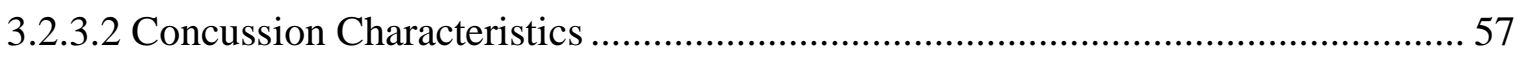

3.2.3.3 Concussion Risk............................................................................................... 58

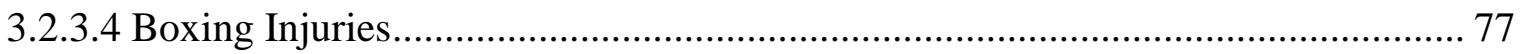

3.2.3.5 Concussion Rates …………………………….......................................... 78

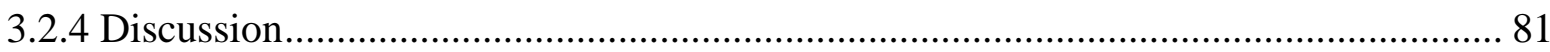

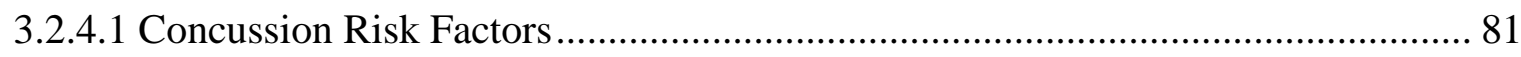

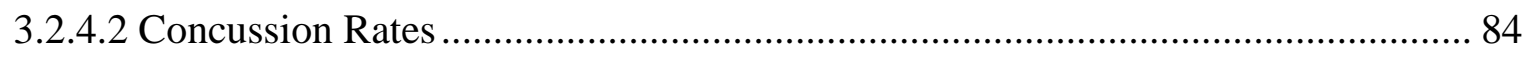

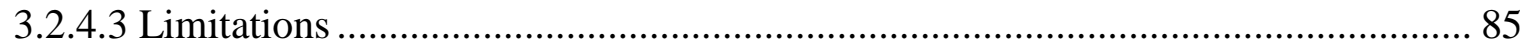

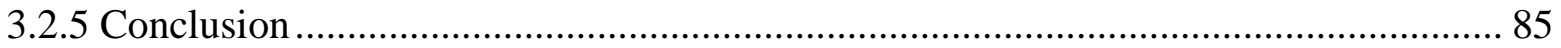

Chapter 3.3 Project 2: Concussion Recovery Trajectories...................................................... 85

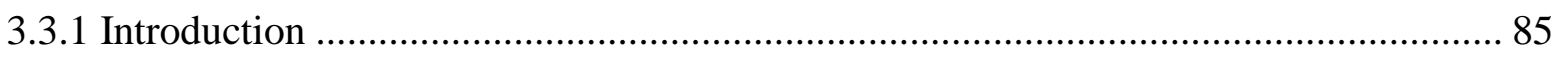

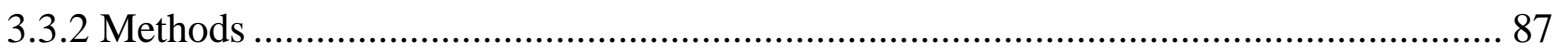

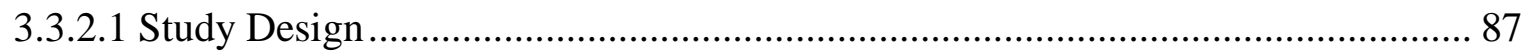

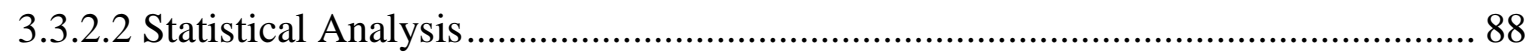

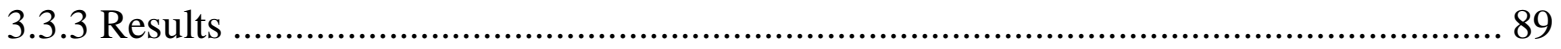

3.3.3.1 Sample Characteristics................................................................................. 89

3.3.3.2 Predictors of Symptom Duration ......................................................................... 91 
3.3.3.3 Predictors of Return to Play Protocol Duration .................................................... 97

3.3.3.4 Mediation of Acute Injury Symptoms by Symptom Resolution Duration .......... 102

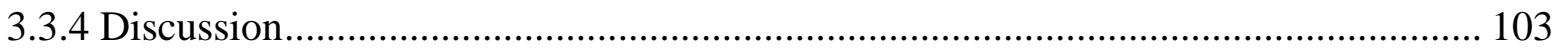

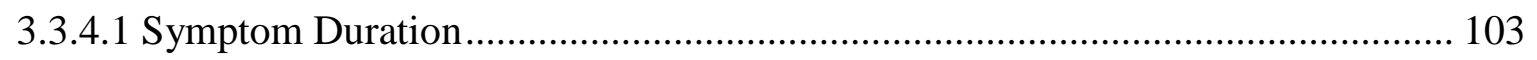

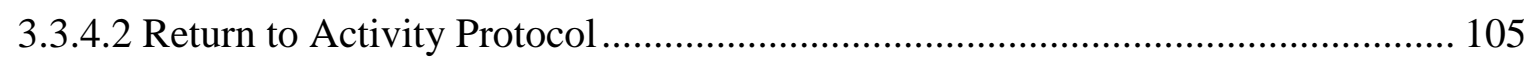

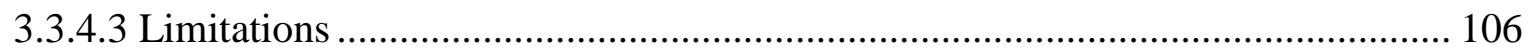

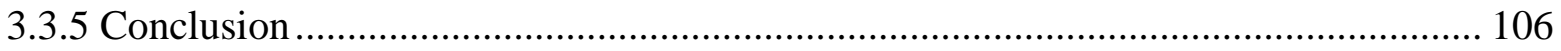

3.4 Project 3: Symptom Predictors of Normal and Abnormal Recovery ................................ 107

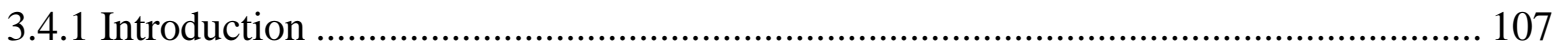

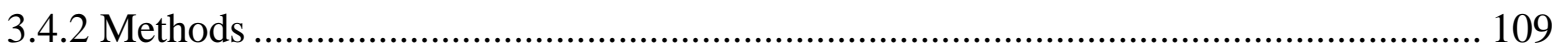

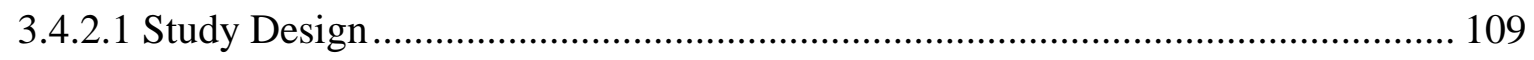

3.4.2.2 Statistical Methods ......................................................................................... 110

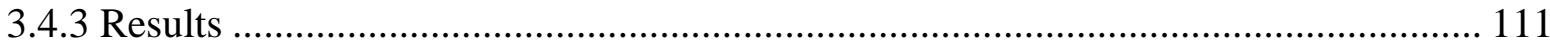

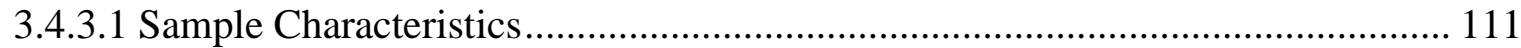

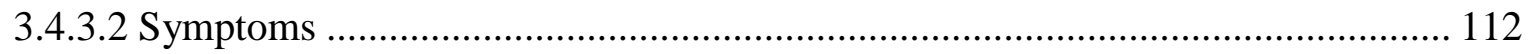

3.4.3.3 Normal versus Abnormal Recovery............................................................. 114

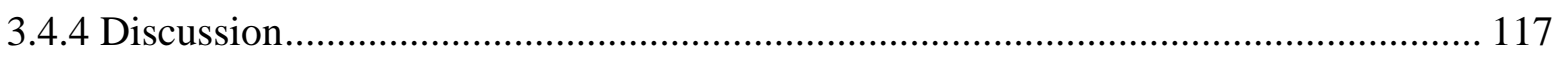

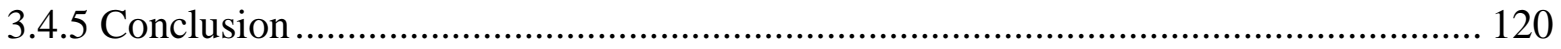

3.5 Project 4: Assessing the bidirectional relationship between psychological symptoms and

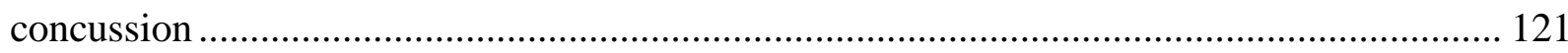

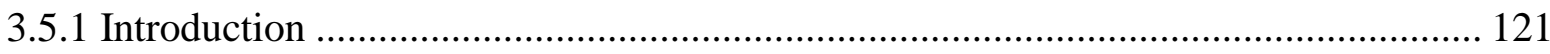

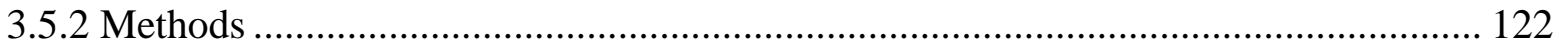

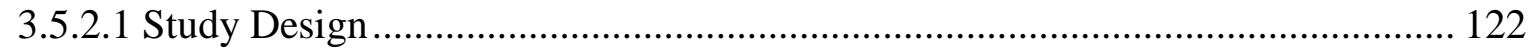

3.5.2.2 Statistical Analysis...................................................................................... 123

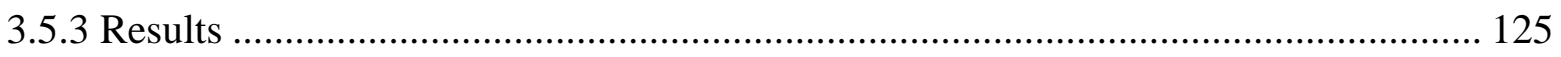

3.5.3.1 Sample Characteristics........................................................................... 125

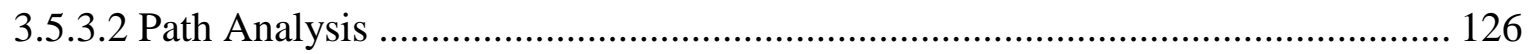

3.5.3.3 Mediation Models ....................................................................................... 129

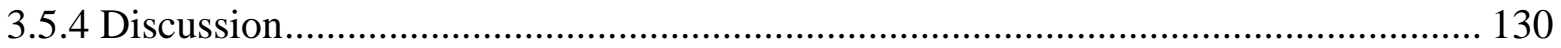

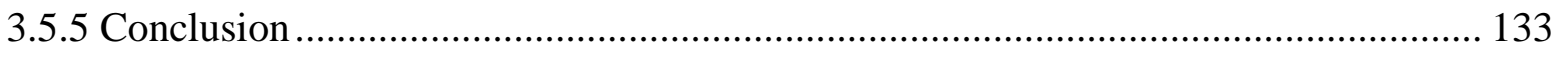

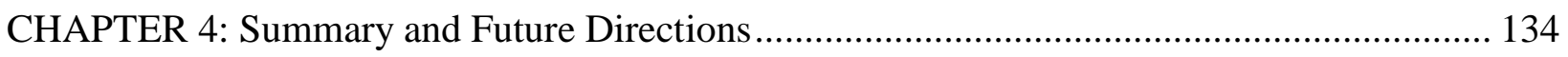

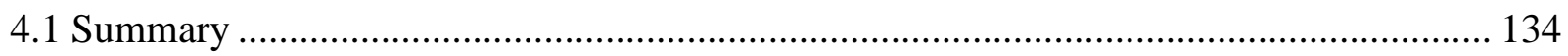

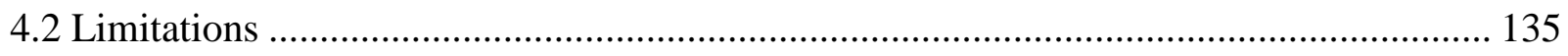




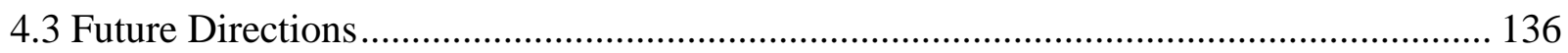

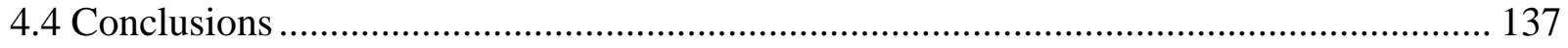

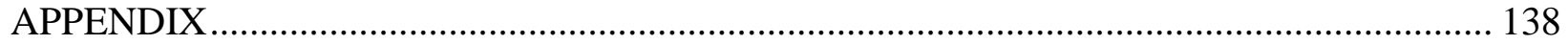

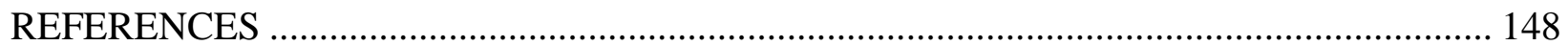




\section{LIST OF TABLES}

Table 1.1.1 Causal Criteria and Limitations in Concussion/mTBI Research ................................. 1

Table 2.3.1. Relationship Between TBI Pathophysiology and Clinical Symptoms ...................... 15

Table 3.1.1. Service Academy Athletic Activities by Competition Level..................................... 46

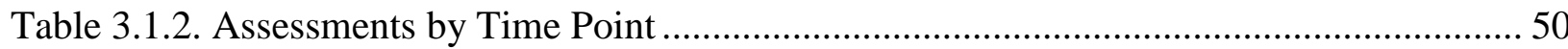

Table 3.2.1a. Univariate Associations with Any Concussion.......................................................... 59

Table 3.2.1b. Univariate Associations with Any Sport-Related Concussion ........................................... 61

Table 3.2.1c. Univariate Association with Any Training-Related Concussion............................... 63

Table 3.2.1d. Univariate Association with Free Time-Related Concussions .................................. 65

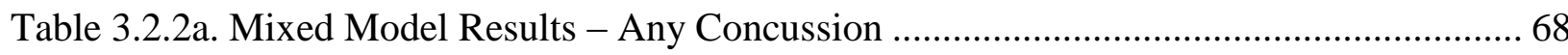

Table 3.2.2b. Mixed Model Results - Sport-Related Concussion ................................................. 70

Table 3.2.2c. Mixed Model Results - Academy Training-Related Concussion............................ 72

Table 3.2.2d. Mixed Model Results - Free Time-Related Concussion ......................................... 73

Table 3.2.2e. Mixed Model Results - Any Concussion among Varsity Athlete Cadets ............... 74

Table 3.2.2f. Mixed Model Results - Any Sport Concussion among Varsity Athletes ................ 76

Table 3.2.3. Concussion rate by sex and sport level status........................................................... 80

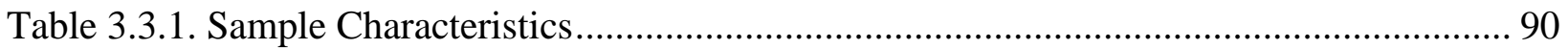

Table 3.3.2. Kaplan Meier Results for Symptom Duration. Median, 95\% Confidence Interval,

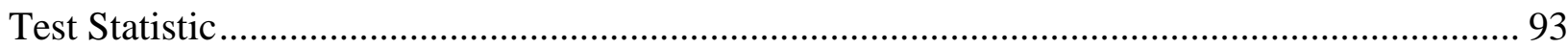

Table 3.3.3. Hazard Ratio and 95\% Confidence Interval - Multivariate Models of Days until

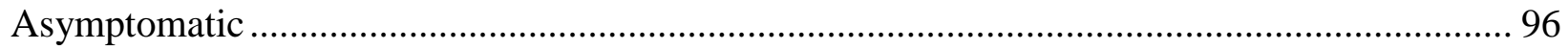

Table 3.3.4. Kaplan Meier Results for RTA Protocol Duration. Median, 95\% Confidence

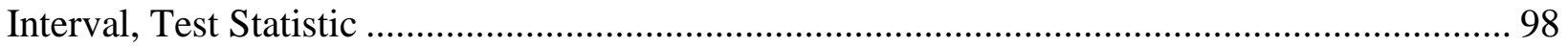

Table 3.3.5. Hazard Ratio and 95\% Confidence Interval - Multivariate Models of RTA Protocol

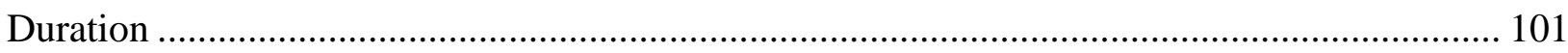

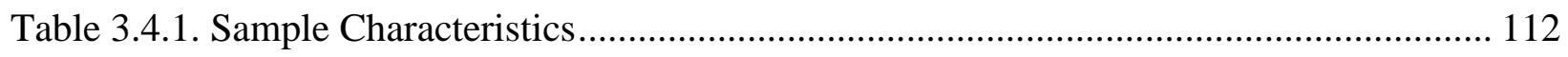

Table 3.4.2. Median and Interquartile Ranges of Symptom Severity at Baseline, within 6 Hours and 24-48 Hours Post-Concussion...................................................................................... 113

Table 3.5.1. Descriptive Statistics by Incident Concussion....................................................... 126 
Table 3.5.2. Path Analysis Model Fit Statistics .............................................................. 126

Table 3.5.3. Path Analysis Unstandardized Parameter Estimates and Standard Errors (SE) ..... 128

Table 3.5.4. Parameter Estimates and Standard Errors (SE) from Iacobucci Mediation Method

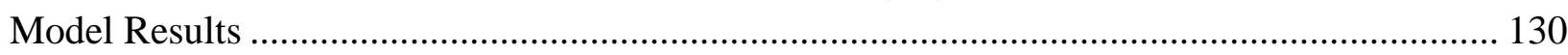




\section{LIST OF FIGURES}

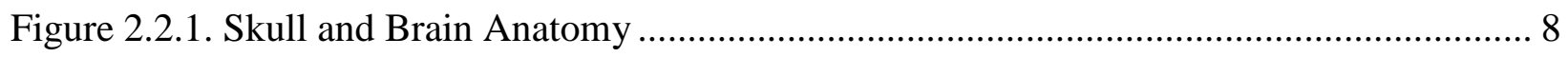

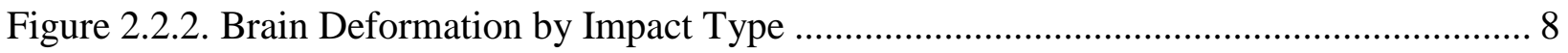

Figure 2.2.3. Angular versus Linear Acceleration ............................................................ 9

Figure 2.2.4. Linear and Angular Average Concussive Magnitudes ....................................... 11

Figure 2.1.1. Summary of Pathophysiology after Traumatic Brain Injury ............................... 17

Figure 2.4.1. Concussion Prevalence by Age and Year........................................................ 19

Figure 2.4.2. Collegiate Concussion and ACL Injury Rates over Time .................................. 21

Table 2.4.1. Concussion Rates (per 10,000 AE) and 95\% Confidence Intervals by Sport and Year

Figure 2.5.1. Components of Concussion Recovery.......................................................... 27

Figure 2.5.2. Comparison of Baseline and Post-Concussion Symptom Prevalence.................... 30

Figure 2.5.3. Summary of Recovery-Related Factors......................................................... 33

Figure 2.6.1. Depression Diagnosis by Concussion History ................................................. 39

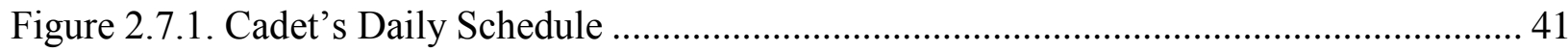

Figure 2.7.2. Academic Performance across Cadets and Non-Cadets..................................... 42

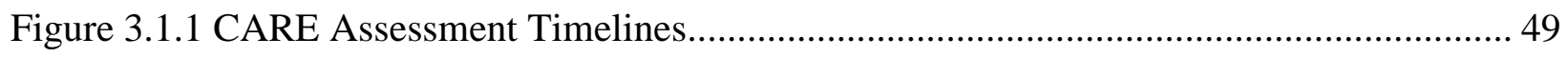

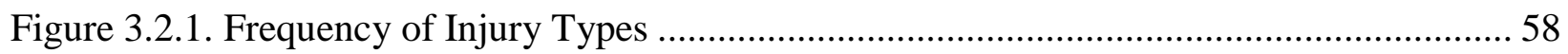

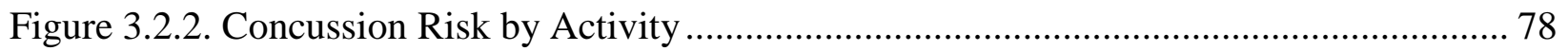

Figure 3.3.1. Frequency of Concussion Types ............................................................. 91

Figure 3.3.2. Kaplan Meier Plots of Days until Asymptomatic among All Concussions. Survival

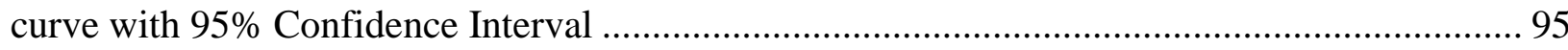

Figure 3.3.4. Mediation Models of RTA Protocol Duration................................................ 102

Figure 3.4.1. SCAT Symptom Prevalence within 6 and 24-48 Hours Post-Concussion........... 114

Figure 3.4.2. CART Results Categorizing Normal and Abnormal Recovery Using 24-48 Hour

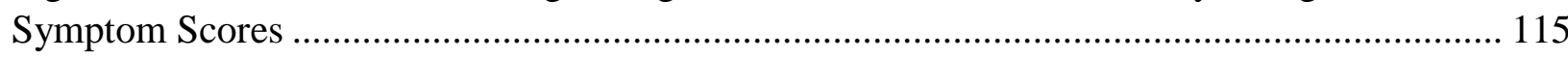

Figure 3.4.3. CART Results Categorizing Normal and Abnormal Recovery Using 24-48 Hour

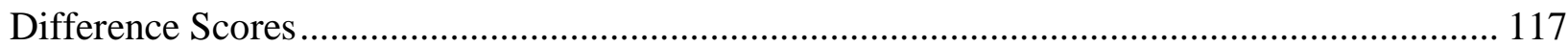

Figure 3.5.1. Hypothesized Model of Concussion and Psychological Symptoms ................... 124 
Figure 3.5.2. Path Analysis Results in Standardized Parameter Estimates 


\section{LIST OF ABBREVIATIONS}

$\begin{array}{ll}\text { Abbreviation } & \text { Definition } \\ \text { AA } & \text { Angular Acceleration } \\ \text { ACL } & \text { Anterior Cruciate Ligament } \\ \text { ACT } & \text { American College Testing } \\ \text { ADD } & \text { Attention Deficit Disorder } \\ \text { ADHD } & \text { Attention Deficit Hyperactivity Disorder } \\ \text { AE } & \text { Athlete Exposure } \\ \text { AFT } & \text { Aerobic Fitness Test } \\ \text { ARC } & \text { Advanced Research Core } \\ \text { ATP } & \text { Adenosine triphosphate } \\ \text { BESS } & \text { Balance Error Scoring System } \\ \text { BSI-18 } & \text { Brief Symptom Inventory - 18 } \\ \text { CARE } & \text { Concussion, Assessment, Research and Education } \\ \text { CART } & \text { Classification and Regression Tree } \\ \text { CFI } & \text { comparative fit index } \\ \text { CI } & \text { Confidence Interval } \\ \text { CMR } & \text { Cerebral Metabolic Rate } \\ \text { CSC } & \text { Clinical Science Center } \\ \text { DoD } & \text { Department of Defense } \\ \text { DTI } & \text { diffusion tensor imaging } \\ \text { DVBIC } & \text { Defense and Veterans Brain Injury Center } \\ \text { ED } & \text { Emergency Department } \\ \text { FA } & \text { fractional anisotropy } \\ \text { GPA } & \text { Grade Point Average } \\ \text { HR } & \text { Hazard Ratio } \\ \text { HRPO } & \text { Human Research Protections Office } \\ \text { ICD } & \text { International Classification of Disease } \\ \text { IQR } & \text { Inter-quartile range } \\ \text { IRB } & \text { Institutional Review Board } \\ \text { K+ } & \text { Potassium } \\ \text { LA } & \text { Linear Acceleration } \\ \text { LOC } & \text { Loss of Consciousness } \\ & \end{array}$




\begin{tabular}{ll} 
LR & Log Rank \\
M & Mean \\
Mdn & Median \\
mTBI & Mild Traumatic Brain Injury \\
$\mathrm{n}$ & Subset Sample Size \\
N & Total Study Sample Size \\
Na & Sodium \\
NAA & N-Acytlapspartate \\
NCAA & National Collegiate Athletic Association \\
NFL & National Football League \\
OEF & Operation Enduring Freedom \\
OIF & Operation Iraqi Freedom \\
OND & Operation New Dawn \\
OR & Odds Ratio \\
PFE & Personal Fitness Exam \\
PFE & Physical Fitness Exam \\
PFT & Physical Fitness Test \\
Pr & Probability \\
PTA & Post-Traumatic Amnesia \\
RGA & Retrograde Amnesia \\
RMSEA & root mean square error of approximation \\
RR & Relative Risk or Rate Ratio \\
RTA & Return to Activity \\
RTP & Return to Play \\
SAC & Standardized Assessment of Concussion \\
SCAT & Standardized Concussion Assessment Tool \\
SD & Standard Deviation \\
SE & Standard Error \\
SEM & Standard Error of the Mean \\
SEM & Structural Equation Modeling \\
SFWP & Symptom Free Waiting Period \\
SRC & Sport Related Concussion \\
SRMR & standardized root mean square residual \\
TBI & Traumatic Brain Injury \\
USAFA & United States Air Force Academy \\
USCGA & United States Coast Guard Academy \\
USMA & United States Military Academy \\
USNA & United States Naval Academy \\
Wxn & Wilcoxon \\
& \\
\hline
\end{tabular}




\begin{abstract}
Background

Concussion, or mild traumatic brain injury, affects 42 million individuals worldwide each year. Most research to date has focused concussion risk, recovery, and outcomes across groups. Furthermore, most studies have been composed of males, varsity athletes, evaluated a single concussion type (e.g. sport versus non-sport related), or had a cross-sectional design.
\end{abstract}

\title{
Objective
}

The objective of the current study is to leverage a prospective multisite prospective study of concussion to identify individual characteristics associated with concussion risk and recovery. Additionally, the relationship between concussion and psychological symptoms will be elucidated to determine whether concussion influences subsequent psychological symptoms or if psychological symptoms also influence concussion risk.

\section{Methods}

Cadets enrolled in the Concussion Assessment, Research and Education Consortium were utilized in this series of dissertation projects. Linear mixed models were implemented to identify demographic and individual factors associated with concussion risk. Next, survival analyses identified factors related to symptom and return to activity protocol durations. Further exploring the relationship between symptom and recovery, Classification and Regression Tree analysis was used to classify normal and abnormal post-concussion symptom durations based on individual acute concussion symptoms. Finally, path analysis evaluated the relationship between concussion and psychological symptoms. 


\section{Results}

Individual risk factors for concussion in included female sex, varsity athlete status, freshmen, having a previous concussion, headaches, ADD/ADHD, and greater BSI-18 somatization symptoms. Each of these factors were associated with a 1.02-2.73 increased concussion risk. Similar risk factors were observed for concussions caused by sport or academy training-related activities. Free time-related concussions were only associated with female sex, freshmen, and previous concussion. These results indicate that females, freshmen, and those with a previous concussion are at greatest risk for an incident concussion of any type.

Separate factors were associated with symptom duration and RTA protocol duration. Thus, symptom duration and RTA protocol durations are likely related but separate entities within total time loss after a concussion. Specifically, the acute number of symptoms had the most consistent and largest effect on recovery durations. Examining individual symptoms, the symptom difference scores between baseline and 24-48 hours post-concussion best predicted normal versus abnormal recovery. Specific symptoms classifying normal versus abnormal recovery were balance problems, dizziness, trouble falling asleep, nausea, and neck pain.

Finally, the relationship between psychological symptoms and concussion was examined. Results demonstrated that baseline somatization had the strongest association with subsequent concussion and future somatization symptoms. Moreover, an incident concussion was associated with lower depression, anxiety, and somatization symptoms, albeit the size of these effects were small.

\section{Conclusion}

Overall, results indicate that there is a constellation of individual and individual factors associated with concussion risk and recovery. In particular, specific individual symptoms predict normal and abnormal recovery. The individual symptoms may represent the most informative symptoms related to concussion recovery and also points of clinical intervention. Additionally, there is a bidirectional relationship between concussion and psychological symptoms which encourages future studies to account of baseline psychological symptoms or mental health. 


\section{CHAPTER 1: Introduction}

\subsection{Statement of the Problem}

Concussion, or mild traumatic brain injury (mTBI), is a growing concern for athletes and the military. Concussion rates among athletes have increased over the last 20 years (Hootman, Agel, \& Dick, 2007; Zuckerman et al., 2015) and has become the signature injury of recent military conflicts (Tanielian et al., 2008). Moreover, while concussion injuries have increased with the occurrence of Operation Enduring Freedom (OEF), Operation Iraqi Freedom (OIF), and Operation New Dawn (OND), the majority of concussions occur within the continental United States(Cameron et al., 2012). Thus, the majority concussion injuries among military service members have similar mechanisms to civilian injuries.

Not only is the occurrence of concussion increasing, but there is growing evidence of negative cognitive and psychological effects after a concussion. Studies of varsity collegiate football players show that most players reach clinical symptom resolution within 7-10 days of injury (McCrea et al., 2005; McCrea et al., 2003). Similar results were found in studies of collegiate athletes in a variety of sports (Broglio, Macciocchi, \& Ferrara, 2007; Zuckerman et al., 2012). Each study was conducted on varsity collegiate athletes, and the majority of samples were male. Two studies at a single service academy studied recovery time after boxing-related concussion (Bleiberg et al., 2004; Warden et al., 2001). Both studies on cadets were all male and look at recovery time up to fourteen days post-injury 
(Bleiberg et al., 2004; Warden et al., 2001). Consequently, there is a dearth of information related to concussion recovery among non-collegiate varsity athletes, females, and cadets.

While many individuals recover from a concussion within 7-10 days, there is increased risk for mental health difficulties later in life. Brain areas susceptible to concussion (Aare $\&$ Kleiven, 2007) and structural and functional changes post-concussion (Asken, DeKosky, Clugston, Jaffee, \& Bauer, 2017; Johnson et al., 2012; Meier, Bellgowan, Bergamino, Ling, \& Mayer, 2016) overlap with neural substrates of mood and anxiety disorders (Liao et al., 2013; Whalley, Rugg, Smith, Dolan, \& Brewin, 2009). Consequently, concussion may make the brain more susceptible to mood and anxiety disorders. Studies of retired professional football players observed dose-response relationships between concussion history and current or previous depression diagnosis (Guskiewicz, Marshall, et al., 2007; Kerr, Marshall, Harding, \& Guskiewicz, 2012). Furthermore, studies of young adults (Vynorius, Paquin, \& Seichepine, 2016) and post-deployment service members (Stein et al., 2015) have also found increased depression symptoms (Vynorius et al., 2016) and likelihood for mood (Stein et al., 2015) and anxiety disorders (Stein et al., 2015) after a concussion. While there appears to be an association between concussion and mental health, previous study design limit the ability to assess the causal role of concussion on subsequent mental health.

Within epidemiology, there are proposed criteria to establish causality. Causation is the concept that a certain exposure is necessary and sufficient to induce a subsequent outcome (Parascandola \& Weed, 2001). To establish whether an exposure results in a certain outcome, Hill outlines certain relationship criteria to be evaluated (Table 1.1.1)(Rothman, 2012).

Table 1.1.1 Causal Criteria and Limitations in Concussion Research

\begin{tabular}{|l|l|l|}
\hline Criteria & Definition & $\begin{array}{l}\text { Limitations in Concussion/mTBI } \\
\text { Research }\end{array}$ \\
\hline Strength & $\begin{array}{l}\text { The degree of } \\
\text { association between } \\
\text { exposure and outcome }\end{array}$ & $\begin{array}{l}\text { Concussion history is often self-reported } \\
\text { with variability in accuracy(Kerr et al., } \\
\text { 2015) }\end{array}$ \\
\hline Consistency & $\begin{array}{l}\text { The repeatability of the } \\
\text { association and } \\
\text { generalizability }\end{array}$ & $\begin{array}{l}\text { Mixed results in imaging(Shenton et al., } \\
\text { 2012) and outcomes(Manley et al., } \\
\text { 2017) post-concussion/mTBI }\end{array}$ \\
& $\begin{array}{l}\text { The exposure precedes } \\
\text { the outcome }\end{array}$ & $\begin{array}{l}\text { Many studies are cross-sectional } \\
\text { assessing concussion/mTBI and } \\
\text { outcome at same time(Carroll, Cassidy, } \\
\text { Holm, Kraus, \& Coronado, 2004) }\end{array}$ \\
\hline
\end{tabular}




\begin{tabular}{|l|l|l|}
\hline Biological Gradient & $\begin{array}{l}\text { A dose-response } \\
\text { relationship }\end{array}$ & $\begin{array}{l}\text { Preliminary studies have shown } \\
\text { increased likelihood of depression with } \\
\text { increasing number of } \\
\text { concussions(Guskiewicz, Marshall, et } \\
\text { al., 2007; Kerr et al., 2012) }\end{array}$ \\
\hline Plausibility & $\begin{array}{l}\text { There are biological } \\
\text { underpinnings of } \\
\text { association between } \\
\text { exposure and outcome } \\
\text { concussion/mTBI(Aare \& Kleiven, } \\
\text { 2007) (Asken, DeKosky, et al., 2017; } \\
\text { Johnson et al., 2012; Meier et al., 2016) } \\
\text { overlap with areas implicated in mood } \\
\text { and anxiety disorders(Liao et al., 2013; } \\
\text { Whalley et al., 2009) }\end{array}$ \\
& $\begin{array}{l}\text { The manipulation of } \\
\text { induce concussion/mTBI in humans }\end{array}$ \\
\hline Experimental Evidence \\
exposure changes
\end{tabular}

Concussions are relatively rare events. In football studies of head impacts, concussions are associated with a small proportion of head impacts (Guskiewicz \& Mihalik, 2011). In a study of high school football impacts, 33 concussions occurred in over 150,000 recorded head impacts (Broglio, Lapointe, O'Connor, \& McCrea, 2017). Additionally, while certain sports and activities are associated with increased concussion risk (Zuckerman et al., 2015), injuries can occur in any sport and activity (Hainline, 2016). A rare but widespread incidence makes prospective studies difficult. Consequently, many studies of concussion have been case-control or cross-sectional. In these study designs, it is difficult to establish that exposure (i.e. concussion) precipitated the outcome of interest (e.g. depression).

Another major limitation to establishing causality within concussion research is sample size. Sample sizes in concussion studies have typically been small and may lead to misleading conclusions (Carroll et al., 2004). Moreover, small sample sizes limit the ability to control for confounders and assess the strength and consistency of the relationship between concussion and outcome. There are many possible individual and environmental confounders of concussion and outcome (Abrahams, Fie, Patricios, Posthumus, \& September, 2014; Iverson et al., 2017). For example, in linear and logistic regression a simple rule for the number of covariates to include in the model is 10-20 cases per covariate. In studies where there is a small sample size, it is difficult to control for possible confounders.

Concussion research is advancing in order to address the issues outlined above. However, there are key knowledge gaps relating to who, where, when and how concussions occur. These 
questions have particularly remained unaddressed in non-varsity athletes and females. Finally, the relationship between concussion and mental health has not been fully evaluated. Study design limitations have prevented the description of the evolution and direction of the relationship between concussion and mental health. Additionally, it is unclear whether there is a specific role of concussion on mental health or whether other individual and environmental factors confound the relationship.

The proposed series of projects aims to address these knowledge gaps. The study will be a prospective study of concussion among service academy cadets. All cadets, not just varsity athletes will be eligible, expanding concussion research to females and non-varsity athletes. Furthermore, all concussions, not just sport-related injuries, will be captured and followed-up. With 800-4,000 cadets at each academy, our sample size will be large to control for potential confounders. Also, cadets are devoid of many medical comorbidities or hypothesized concussion modifiers. The proposed projects will examine the who, where, when, and how concussions occur and recover. A specific focus will be the relationship between concussion and mental health. The prospective design will enable our team to describe the direction of the relationship and whether concussion is a cause of subsequent mental health.

\subsection{Overarching Aims and Hypotheses}

The purpose of these series of projects is to identify concussion risk factors outside of male varsity collegiate athletes. Secondly, the prospective study design enables the research team to evaluate the causal role of concussion on mental health. These projects will fill critical knowledge gaps and provide more generalizable information due to the inclusion of non-varsity athletes and females.

Aim 1: Identity at-risk groups and activities that are associated with increased likelihood of concussion

Hypothesis 1: Varsity sport cadets will have highest concussion likelihood compared to club and intramural cadets.

Hypothesis 2: Sex will be significantly associated with concussion likelihood, with males having an increased likelihood of concussion. However, among sexcomparable sports (e.g. basketball) females will have a greater concussion likelihood.

Aim 2: Identify injury and individual characteristics associated with recovery duration. 
Hypothesis 1: Acute injury characteristics (e.g. LOC, amnesia, delayed reporting) will be associated with longer recovery trajectories.

Hypothesis 2: Females and non-varsity cadets will have longer recovery durations.

Aim 3: Identify individual symptoms associated with normal and abnormal recovery. Hypothesis 1: Symptoms not typically reported at baseline (e.g. dizziness) will provide the greatest information and be associated with abnormal recovery

Aim 4: Elucidate the relationship between concussion and mental health Hypothesis 1: Anxiety and somatization baseline symptoms will be associated with greater incident concussion risk.

Hypothesis 2: Incident concussion will be associated with increased depression, anxiety, and somatization symptom scores at the following annual evaluation. Hypothesis 3: Concussion will mediate the relationship between symptom scores (anxiety, and somatization) at annual evaluations 


\section{CHAPTER 2: Concussion Review}

\subsection{Concussion Definition}

Traumatic brain injury (TBI) is an injury to the brain resulting from a direct head impact or indirect forces applied to the body (Decuypere \& Klimo, 2012). TBI is a spectrum of injury ranging from mild to severe (Centers for Disease Control and Prevention, 2015). Mild TBI (mTBI) is the most common form of brain injury (Centers for Disease Control and Prevention, 2015) and commonly results from falls, motor vehicle accidents, and sport/recreation activities (Bazarian et al., 2005). For those mTBIs occurring during sport/recreation, the term “concussion" is commonly used (Harmon et al., 2013).

In 2001, the first International Conference on Concussion in Sport was held to design an agreement document on concussion, including a singular definition of concussion. Since 2001, there have been five International Conference on Concussion in Sport conferences. Beginning in 2001 concussion was defined as (Aubry et al., 2002):

“... a complex pathophysiological process affecting the brain, induced by traumatic biomechanical forces."

Also outlined by the concussion in sport group were five injury constructs that may or may not be present. These constructs included an impulsive force mechanism, rapid and transient neurological impairment, neuropathological but not structural injury, and may not involve loss of consciousness (Aubry et al., 2002). Since 2001, the original consensus definition has remained relatively consistent, with few minor updates. Most notable are the updates from the most recent 
2016 consensus meeting. During this meeting, concussion was identified to be the same as mTBI. Previously, concussion was thought to be separate from mTBI, or a less severe subset of mTBI (Harmon et al., 2013; McCrory, 2001; McCrory, Meeuwisse, Aubry, Cantu, Dvořák, Echemendia, Engebretsen, Johnston, Kutcher, Raftery, Sills, et al., 2013).

Additionally, the 2016 consensus group added the stipulation that the symptoms observed after an impact must not be explained by anything else (e.g. dehydration) (McCrory et al., 2017). Despite subtle differences in each definition, each of the recent definitions includes the following consistent attributes: a change in brain function, after direct/indirect force to brain, may involve loss of consciousness, and may include neurological or cognitive dysfunction and normal neuroimaging.

\subsubsection{Summary}

For this study, a concussion is defined using a consensus definition produced by a U.S. Department of Defense funded evidence-based guidelines group and endorsed by the NCAA Concussion Management Guidelines 2014 (Carney et al., 2014). This definition is based on a systematic review of the literature (Carney et al., 2014) and agreed upon by the governing bodies (DoD and NCAA) which oversee the study's population. This consensus definition defines concussion as a change in brain function following a force to the head, which may be accompanied by temporary loss of consciousness, but is identified in awake individuals with measures of neurologic and cognitive dysfunction.

\subsection{Mechanics of Concussion}

Concussion results from a direct or indirect blow to the head resulting in transient neurological impairment and neuropathological symptoms (McCrory et al., 2017). Due to underreporting (LaRoche, Nelson, Connelly, Walter, \& McCrea, 2016; McCrea, Hammeke,

Olsen, Leo, \& Guskiewicz, 2004), transient symptoms (Harmon et al., 2013), delayed symptom onset (Duhaime et al., 2012), and few concussions occurring with overt signs such as loss of consciousness (McCrory et al., 2017), concussions are often difficult to detect and diagnose (Broglio \& Guskiewicz, 2014; Kelly \& Rosenberg, 1997). Clinical tools have been developed to assess symptoms, balance, eye movement, pupil reactivity, electrophysiology, and cognition (Kutcher et al., 2013). While these tools provide clinicians with a clinical representation of concussion they are not diagnostic and typically require the athlete to report the suspected injury. To overcome these limitations and relieve the athlete of reporting responsibility, head impact 
biomechanics have been investigated to determine the kinematic signature of a concussion (Guskiewicz \& Mihalik, 2011; O'Connor, Rowson, Duma, \& Broglio, 2017).

Acceleration represents the rate of change in velocity and in this review resultant linear accelerations (LA) and resultant angular accelerations (AA) of the head will be reported. Resultant LA is the vector sum magnitude of the three-dimensional linear accelerations of the skull resulting from an impact. LA is measured in gravitational units $(\mathrm{g})$, which is equal to the acceleration due to gravity $\left(\sim 9.81 \mathrm{~m} / \mathrm{s}^{2}\right)$. Similarly, AA is the vector sum of the threedimensional angular accelerations of the skull resulting from an impact and is measured in units of $\mathrm{rad} / \mathrm{s}^{2}$. Linear and angular acceleration are tightly correlated to one another (Pellman, Viano, Tucker, Casson, \& Committee on Mild Traumatic Brain Injury, 2003; Rowson et al., 2012). The relative magnitude of LA and AA is dependent on how far away the impact is from the center of gravity of the head. Vectors further away from the center of gravity create greater AA relative to LA. Alternatively, impacts in line with a head's center of gravity (e.g. radial) translate to more LA (Stemper \& Pintar, 2014). Since few impacts are perfectly aligned with the head's center of gravity, most impacts are composed of both linear and rotational components(Kleiven, 2013). Thus, in most instances, lowering LA magnitude lowers AA.

Each head impact is a combination of LA and AA. The human brain sits within the skull surrounded by meninges and encompassed by cerebral spinal fluid (Figure 2.2.1). The brain moves independently of the skull but is tethered at its base by the brainstem, blood vessels, and membranes (Feng et al., 2010). MRI studies of in vivo linear (Feng et al., 2010) and angular impacts (Sabet, Christoforou, Zatlin, Genin, \& Bayly, 2008) demonstrate brain deformation during mild impacts. The brain is suspended within the skull; when the linear impact is applied to the forehead, the brain rotates at the attachment point at the base of the skull (Feng et al., 2010) (Figure 2.2.2a). The deformation effects are most notable on the superior cortical surface, basal frontal regions, and occipital cortex. During an angular impact, the cortex rotates around the brainstem and base of the brain. The resulting torque is transmitted into brain shear deformation (Figure 2.2.2b). Results replicated findings from imaging of a viscoelastic gel that served as a brain surrogate (Figure 2.2.2c). Another study used finite element modeling to reconstruct 24 American football impacts that resulted in concussion (Zhang, Yang, \& King, 2004). Initially, the shear stresses were observed in the cortex, they then moved to the midbrain by the time the impact reached peak magnitude (Zhang et al., 2004). Thus, small magnitude 
impacts may have the generated shear forces on the cortical surface, but higher magnitude impacts, those associated with concussion, cause both cortical and subcortical deformation.

Figure 2.2.1. Skull and Brain Anatomy

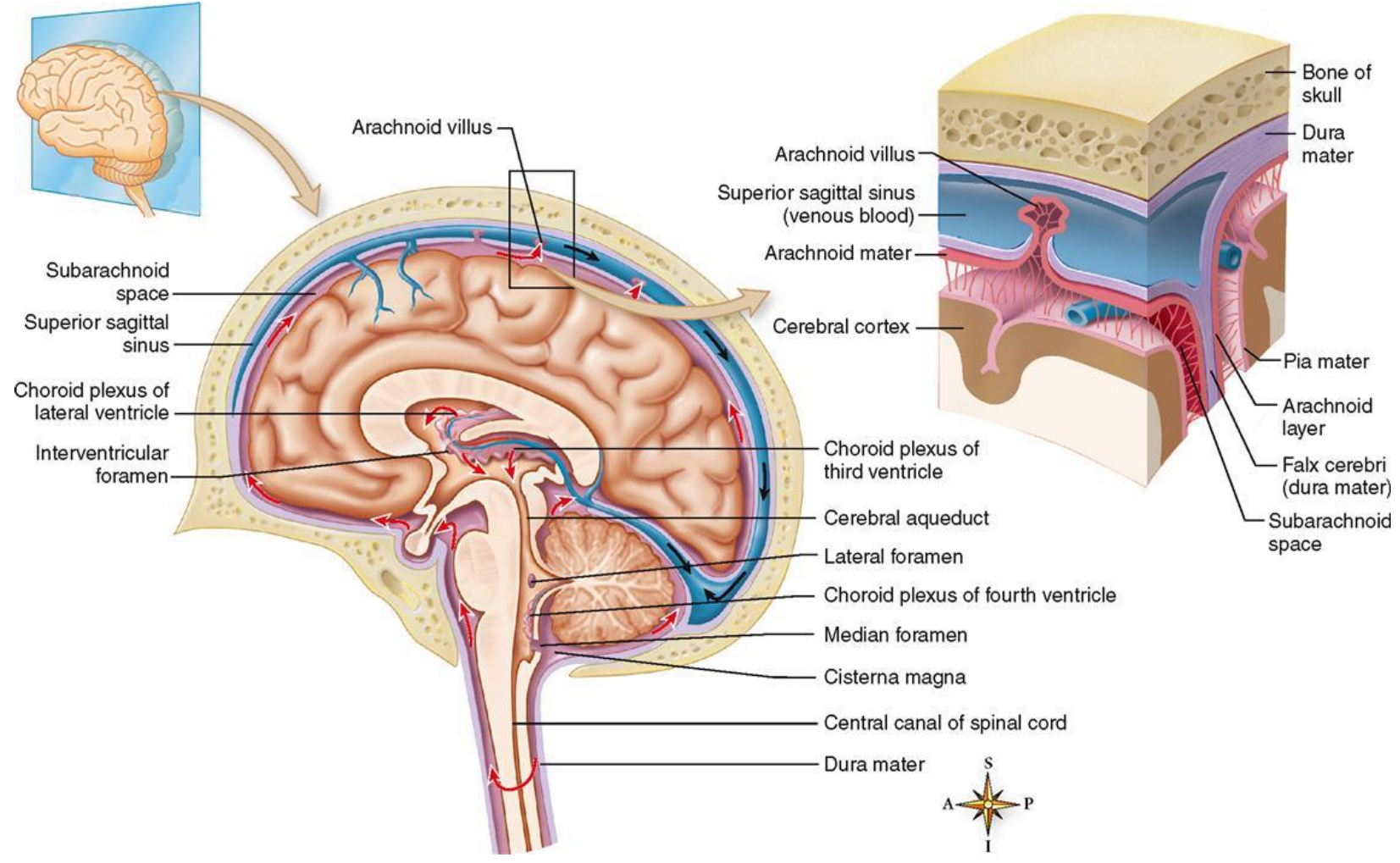

Figure 2.2.2. Brain Deformation by Impact Type
A. Linear Acceleration (Feng 2010)
B. Angular Acceleration (Sabet 2008)
C. Angular Acceleration - Brain Surrogate

(Bayly 2007)
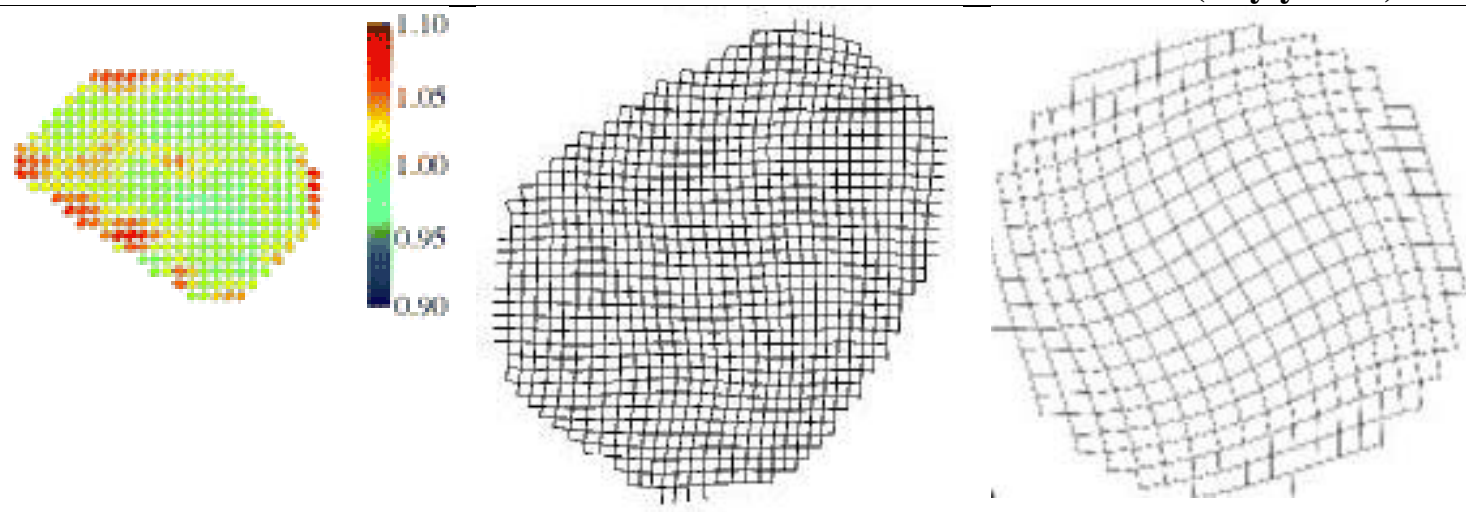
The degree of brain movement relative to the skull is dependent on the brain size, shape, and impact direction (Zhao, Ruan, \& Ji, 2015). Larger brains are associated with increased pressure gradients (Zhao et al., 2015), increasing injury risk. Inertia, the concept that an object in motion will stay in motion, is dependent on that object's mass. The greater the brain mass, the greater inertia, and movement within the skull. Thus, for the same impact magnitude, a larger brain would sustain more damage (Stemper \& Pintar, 2014). The importance of brain size is notable as it may explain some of the individual variability observed in concussion biomechanics (Guskiewicz \& Mihalik, 2011).

Consistently, angular acceleration is associated with brain deformation associated with concussion while linear acceleration is associated with skull fracture (Kleiven, 2013) (Figure 2.2.3). The brain has high water content, making it relatively incompressible (Libertiaux, Pascon, $\&$ Cescotto, 2011). Thus, a linear force has little effect on brain deformation. Modeling of a blunt head impact demonstrated that linear acceleration only contributed $10 \%$ to the total strain while angular acceleration composed $90 \%$ of the brain tissue's strain (Zhang, Yoganandan, Pintar, \& Gennarelli, 2006). Angular acceleration creates diffuse axonal injury due to widespread brain tissue strain (Stemper \& Pintar, 2014).

Figure 2.2.3. Angular versus Linear Acceleration

Impact direction Kinematics

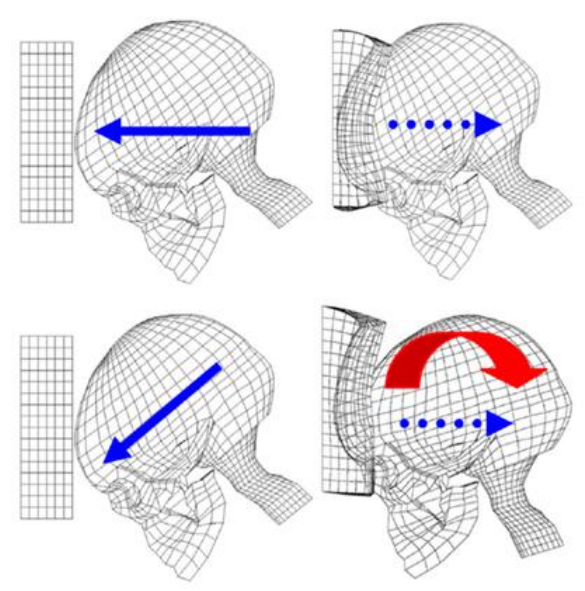

Skull stress

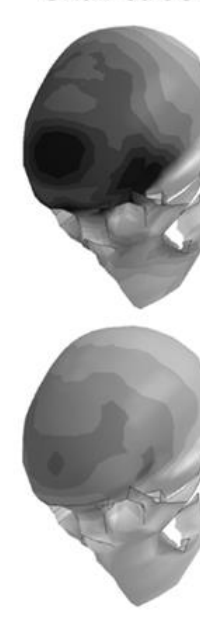

Brain strain

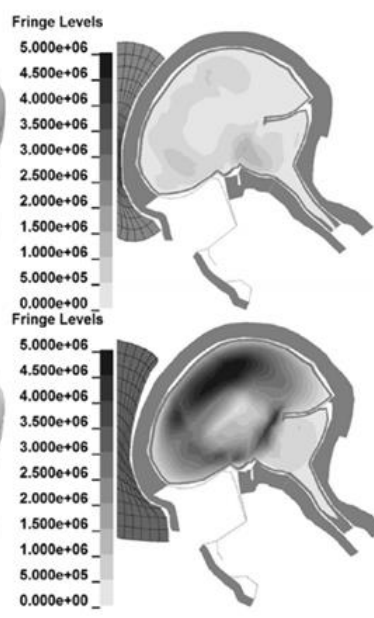

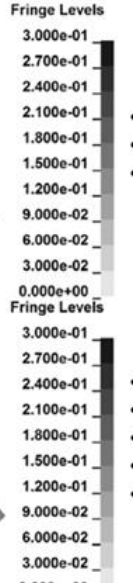

Injury types

- Skull fracture

- Contusion (secondary)

- Epidural Hematoma

From Klieven et al.(Kleiven, 2013)

Determining the mechanics of brain injury is not a recent area of investigation. In 1943, Holbourn et al. (Holbourn, 1943) theorized on the mechanics of head injuries, focusing on rotational forces. Subsequent primate work suggested that while AA may produce diffuse and 
focal injury, LA produces only focal injury (Ommaya, 1985). Since then, LA and AA have been posited to influence concussive injury (Guskiewicz \& Mihalik, 2011; Post \& Hoshizaki, 2015; Rowson, Brolinson, Goforth, Dietter, \& Duma, 2009) with LA and AA thought to cause brain injury through respective pressure gradients and shearing stress (King, Yang, Zhang, \& Hardy, 2003; Kleiven, 2013). While there is debate regarding whether LA, AA, or combined LA and AA influence concussion risk, neither occur in isolation (Kleiven, 2013).

Modeling studies generate hypothetical injury thresholds. In vivo measurement of head impacts using accelerometers within the helmet or device affixed to the head provide real-time and real-world impact measures (O'Connor et al., 2017). Normative median linear acceleration magnitudes in collegiate football fall between 15-19g and angular values 872-1017 rads/ $/ \mathrm{s}^{2}$. Comparatively, collegiate ice hockey players have lower median linear magnitudes (15.0-15.7 g) but higher angular magnitudes (1211-1630 rads/s²) (Wilcox, Beckwith, et al., 2014). While female collegiate soccer players appear to have greater median linear (31.2 g) and angular (6429 $\mathrm{rads} / \mathrm{s}^{2}$ ) magnitudes (McCuen et al., 2016) than football and ice hockey players, different measurement devices and error rates may account for the differences in magnitudes. Comparison across studies using different head impact measurement devices should be made with care (O'Connor et al., 2017).

A review of concussive head impact data for collegiate athletes yielded 138 concussive events collected across collegiate football, and collegiate male and female ice hockey (O'Connor et al., 2017). Football concussive impacts are associated with linear magnitudes ranging from 69.7-145g (Beckwith et al., 2013; Duhaime et al., 2012; Funk, Rowson, Daniel, \& Duma, 2011; Guskiewicz, Mihalik, et al., 2007); while within ice hockey, linear acceleration ranges from 30.7 to $31.7 \mathrm{~g}$ in an all-male sample $(\mathrm{n}=2)$ and 30.4 to $52.2 \mathrm{~g}$ in an all-female sample $(\mathrm{n}=4)$ (Brainard et al., 2012; Wilcox, Beckwith, et al., 2014; Wilcox et al., 2015; Wilcox, Machan, et al., 2014). Rotational values averaged for male ice hockey players ranged from 1307 to $5419 \mathrm{rad} / \mathrm{s}^{2}$ and averaged $4030 \mathrm{rad} / \mathrm{s}^{2}$ for females (Brainard et al., 2012; Wilcox, Beckwith, et al., 2014; Wilcox et al., 2015; Wilcox, Machan, et al., 2014). A summary of average concussive magnitudes across sport and age are presented in Figure 2.2.4. Regarding concussive magnitudes, older athletes sustain greater magnitude impacts. Collegiate athletes sustain concussions associated with larger linear and angular accelerations, compared to high school or youth athletes (Figure 5). The 
increased magnitudes may indicate higher tolerances of older athletes or the greater accelerations experienced due to increased size, speed, and skill of players at older age groups.

Figure 2.2.4. Linear and Angular Average Concussive Magnitudes

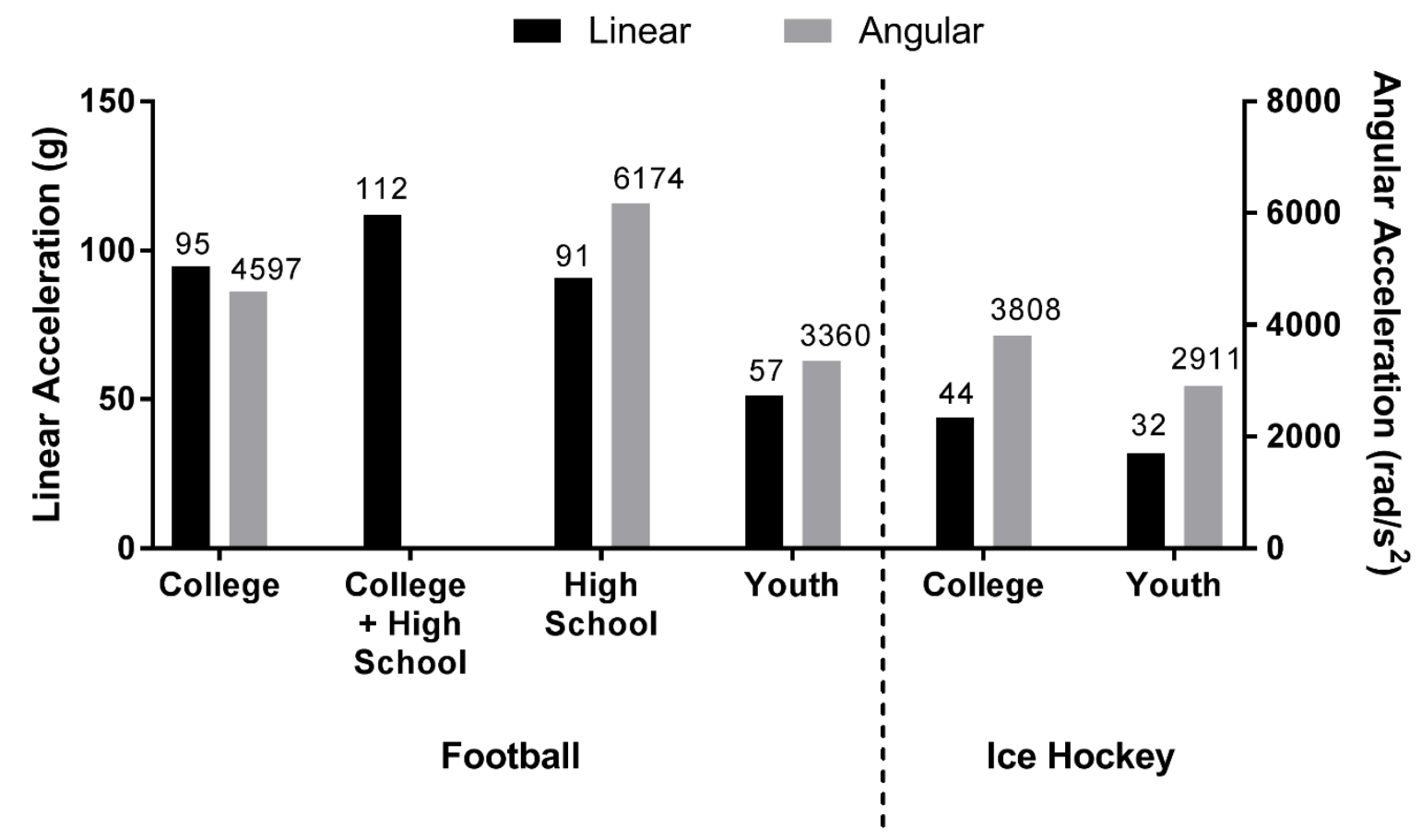

Individual concussive impact variables were extracted from multiple studies and the means are presented here. The number of concussive cases for each variable are as follows: Football: College linear $(n=82)$, college angular $(n=105)$, high school + college linear $(n=45)$, high school linear $(n=25)$, high school angular $(n=24)$, youth linear $(n=6)$, youth angular $(n=4)$. Ice Hockey: College linear $(n=6)$, college angular $(n=6)$, youth linear $(n=1)$, youth angular $(n=1)$.

Note: Adapted from O'Connor 2017 (O'Connor et al., 2017)

\subsubsection{Summary}

While no injury threshold exists, research has found criteria that increase risk for concussion. Impact magnitude, location, and impact density have been used to demonstrate increased concussion risk. Future research to expand to non-helmeted sports and sex-comparable sports to elucidate whether one sex is more biomechanically susceptible to injury. Additionally, individual characteristics, such as brain size should be taken in to account to help explain interindividual variability. Similarly to sideline tests, head impact biomechanics are not diagnostic. 
However, since greater magnitude impacts are more commonly associated with concussion, activities with greater head impact frequency and magnitude may have greater concussion risk.

\subsection{Pathophysiology}

The mechanical forces transmitted to the brain cause physiological perturbations that manifest as clinical symptoms post-concussion (Giza \& Hovda, 2014). The mechanical perturbation of the neuron membrane depolarizes the membrane and is thought to force open ion channels (Katayama, Becker, Tamura, \& Hovda, 1990; Vagnozzi et al., 1999). Open ion channels enabling the efflux of $\mathrm{K}+$, influx of calcium (Ca2+), and glutamate release (Katayama et al., 1990; Vagnozzi et al., 1999).

In response to the ion imbalance the neuron attempts to regain homeostasis (Kawamata, Katayama, Hovda, Yoshino, \& Becker, 1992). The neuron increases glucose utilization up to $181 \%$ (Kawamata et al., 1992) in order to fuel sodium/potassium pumps to regain the neurons resting membrane potential. Energy (ATP) must be used in order to pump potassium back into the cell via the sodium potassium pump to regain the proper sodium/potassium concentrations (Farkas, Lifshitz, \& Povlishock, 2006). This energy demanding process rapidly depletes energy stores, despite an initial surge in glucose (Yoshino, Hovda, Kawamata, Katayama, \& Becker, 1991). The initial surge in glucose arrives with the increase in cerebral blood flow. However, after minutes cerebral blood flow decreases to a below normal level.

Glucose oxidation, or the breakdown of glucose into energy, is impaired due to damaged mitochondria. The mitochondria are less efficient in processing glucose due to shunting of excess calcium that entered the neuron (Verweij et al., 1997; Xiong, Gu, Peterson, Muizelaar, \& Lee, 1997) and the degeneration of the mitochondrial membrane (Vagnozzi et al., 1999). The impaired mitochondria attempt to compensate by performing less efficient anaerobic glucose metabolism (Kawamata, Katayama, Hovda, Yoshino, \& Becker, 1995). Following concussion, animal models show increased levels of lactate at 5 minutes post-injury and reach significant increase at 20 minutes post-injury (Meyer, Kondo, Nomura, Sakamoto, \& Teraura, 1970).

$\mathrm{N}$-Acytlapspartate (NAA) is a metabolite specific to the nervous system that is involved in myelination of neurons and is associated with neuroenergetics (Moffett, Ross, Arun, Madhavarao, \& Namboodiri, 2007). The robust and neuron-specific prevalence of NAA in the nervous system has made it a hypothesized TBI marker (Moffett et al., 2007). Reduced NAA levels are associated with neuronal loss (Signoretti et al., 2001) and impaired mitochondrial 
function (Signoretti et al., 2001; Vagnozzi et al., 2007). Animal models of TBI demonstrated that NAA and ATP levels decrease post-injury even in absence of gross structural damage (Signoretti et al., 2001). Thus in the case of concussion, the return of NAA to normal levels indicates the return of energy homeostasis within the brain (Vagnozzi et al., 2010) and is hypothesized to be a marker of true brain recovery (Vagnozzi et al., 2010).

Human studies, using MRS, have shown acute and chronic reductions in NAA (Gardner, Iverson, \& Stanwell, 2014). Within four days of injury, NAA was significantly decreased within the dorsolateral prefrontal cortex and primary motor cortex (Henry, Tremblay, Boulanger, Ellemberg, \& Lassonde, 2010). While some have shown NAA levels return to the levels of nonconcussed around day 30 (Floris et al., 2008; Vagnozzi et al., 2010), Henry and colleagues (Henry et al., 2011) showed that NAA levels remain decreased up to 6 months post injury. Metabolic dysfunction was also shown to persist and be exaggerated if a participant sustained a second concussion within the first two weeks of the first concussion (Floris et al., 2008; Vagnozzi et al., 2010).

Prolonged hypometabolism, chronic energy decoupling, would result in greater oxidative stress and apoptosis. Consequently, not only would neuronal function be perturbed but there would also be structural impairment. In support of this hypothesis, Henry (Henry et al., 2011) found changes in the white matter integrity of the brain. Henry (Henry et al., 2011) observed decreased fractional ansitropy and increased mean diffusivity in the corpus callosum, hippocampus, and cerebellum.

In addition to affecting metabolism in neuron cell body, stretching and sheering forces also affect the axon. These forces alter axon membrane permeability, causing an influx of calcium and deformation of neurofilaments and microtubules (Giza \& Hovda, 2014; Prins, Greco, Alexander, \& Giza, 2013). The damage to the neurofilaments and microtubules impairs intracellular transport and organelles. Impaired cellular transport causes items accumulate at the site of injury, creating a cellular level traffic jam. This accumulation causes swelling of the axon and in more severe injuries contributes to cell death (Barkhoudarian, Hovda, \& Giza, 2016; Giza \& Hovda, 2014). In an animal model using fluid percussion injury, damage was shown across cortical and subcortical axons, but not to the soma or myelin sheaths (Spain et al., 2010). This damage was apparent as soon as 4 hours post-injury and persisted through 6 weeks post injury (Spain et al., 2010). The localization of injury to the axon and not the soma may indicate that 
axons are more susceptible to concussive stretching forces. Thus, lower impact forces may still elicit neuropathology due to axonal damage.

Advanced imaging techniques including diffusion tensor imaging (DTI) and tractography have enabled researchers to assess axonal integrity in humans in vivo. These methods assess the diffusion of water within the axon to determine integrity. If one considers the axon as a cylinder, water is better able to diffuse along the length on the cylinder and is limited by the width of the cylinder. Axons that are damaged allow water to diffuse in more directions, reducing the amount of linear diffusion, or fractional anisotropy (FA). FA ranges from 0 to 1 , with lower values representing freer diffusion and higher values representing diffusion restricted to one direction. Acute imaging of concussion has shown reduced FA was observed in the internal capsule and corpus callosum, two main intra and inter-hemispheric white matter tracts (Arfanakis et al., 2002). Follow-up scans were obtained on two injured subjects, and imaging results at 30 days showed a recovery of white matter integrity indicating that white matter damage from a single concussion was not permanent (Arfanakis et al., 2002). In a larger study of 26 concussed and non-concussed athletes, found a difference acute and sub-acute white matter response (Churchill et al., 2017). FA levels increased with time from injury (Churchill et al., 2017). This study helps explain why previous studies may have found no differences in connectivity, or both increased and decreased FA during acute injury (Asken, DeKosky, et al., 2017). Thus, the brain response is time dependent and future research should strongly consider time from injury in their recruitment and/or analysis measures.

While the acute injury phase has the most heterogeneous results, chronic studies, one year or more removed from injury, have the most consistent findings (Asken, DeKosky, et al., 2017). In a systematic review of DTI findings in concussion, 8 of 12 civilian studies and 7 of 13 studies investigating military concussion found impaired white matter at least one year from injury/exposure (Asken, DeKosky, et al., 2017). A separate review and meta-analysis supported these findings, reporting that acute injury is defined by increase anisotropy followed by decreased anisotropy in chronic stages (Eierud et al., 2014).

While there is heterogeneity within the brain imaging literature, there appears to be growing evidence for white matter damage after concussion. The white matter damage results from the stretching and shearing forces and sometimes may results in cell death. Impaired axons or reduced number of axons is most commonly observed in the corpus callosum, internal 
capsule, and superior longitudinal fasciculus (Narayana, 2017). Frontal lobe areas are common areas of white matter impairment, and may represent an area vulnerable to concussion (Eierud et al., 2014). Many white matter changes seen post injury also correlated with symptoms and cognitive impairment (Narayana, 2017; Spain et al., 2010). With sufficiently impaired brain structure there will also be impaired function that elicits clinical symptoms experienced after concussion.

Perturbed ionic flux, cerebral blood flow, glucose metabolism, axonal injury all have clinical correlates (Giza \& Hovda, 2014). First, the timing of symptom onset varies. Some individuals may experience symptoms immediately after concussion, while others may develop symptoms over the following hours (McCrory, Meeuwisse, Aubry, Cantu, Dvořák, Echemendia, Engebretsen, Johnston, Kutcher, Raftery, Sills, et al., 2013). A possible mechanism for varying symptom onset may be the different timing of membrane permeability. Membrane permeability can occur in three distinct patterns 1) initial perturbations but reseal, 2) enduring perturbation, 3) delayed membrane damage (Farkas et al., 2006).

Beyond symptom onset, metabolic perturbations have also been associated with symptom severity. Acute metabolic changes, decreased NAA, observed within the first 4 days of injury correlated with symptom severity but not neurocognitive performance (Henry et al., 2010). White matter changes also have been associated with the severity of symptoms (Wilde et al., 2008) and reduced neuropsychological performance (Eierud et al., 2014). Giza and Hovda (Giza \& Hovda, 2014) propose clinical correlates for each TBI pathophysiology (Table 2.3.1).

Table 2.3.1. Relationship Between TBI Pathophysiology and Clinical Symptoms

\section{Pathophysiology Clinical Correlate}

Ionic flux Migraine headache, sensitivity to light and sound

Axonal Injury/Impaired Impaired cognition, slowed processing, slowed reaction Neurotransmission time Hypometabolism Vulnerability to second injury Adapted from Giza 2014 (Giza \& Hovda, 2014)

Individuals with a concussion may demonstrate impaired cognitive abilities post-injury. Neurocognitive deficits may not always be apparent due to compensatory mechanisms. While concussion patients may have the same behavioral performance as non-concussed patients, those with concussion require greater cognitive effort to complete the task (Jantzen, Anderson, Steinberg, \& Kelso, 2004). Among patients with a single concussion, experiencing post- 
concussive symptoms, there was reduced percent change in regional cerebral blood flow during a working memory task compared to healthy controls (Chen, Kareken, Fastenau, Trexler, \& Hutchins, 2003). Similarly, McAllister and colleagues (McAllister, Saykin, Flashman, \& Sparling, 1999) observed increased brain activation during difficult working memory tasks, but similar performance when compared to controls (McAllister et al., 1999). Mouse models add to the human work demonstrating impaired learning after concussion, without cell death, indicating that impaired structure was the cause of cognitive impairment (DeFord et al., 2002).

Figure 2.3.1 summarizes a neuron's response to concussion and the metabolic cascade that follows. Immediately after a force is applied to the brain a complex series of microstructural and neurometabolic events occurs. These events are diffuse, taking place throughout the brain, rather than localized to a singular injury site. Mechanical forces stretch neuronal membrane, making it more permeable, disrupting ion flow and neuronal signaling. To restore ion balance, the ATP-dependent sodium/potassium pump works at such a rate that it rapidly depletes energy stores. Unfortunately, the mitochondria are not able to keep up with the ion pump energy demands due to oxidative dysfunction and thus the brain enters a reduced cerebral metabolic state. This neurometabolic cascade presents clinically, by impaired cognition, reaction time, and symptoms (e.g. headache). Age, severity of injury, and interval between injuries have been shown to influence metabolic recovery. While recovery after a single concussion transient there is growing evidence that repeat injuries may result in a more persistent pathologies.

\subsubsection{Summary}

Concussion results from linear and angular forces transmitted to the brain that disrupt normal brain physiology. This pathophysiological response gives rise to the common symptoms experienced after a concussion. Thus, a concussion is the clinical manifestation of microstructural and functional changes in the brain after a traumatic impact. 
Figure 2.1.1. Summary of Pathophysiology after Traumatic Brain Injury

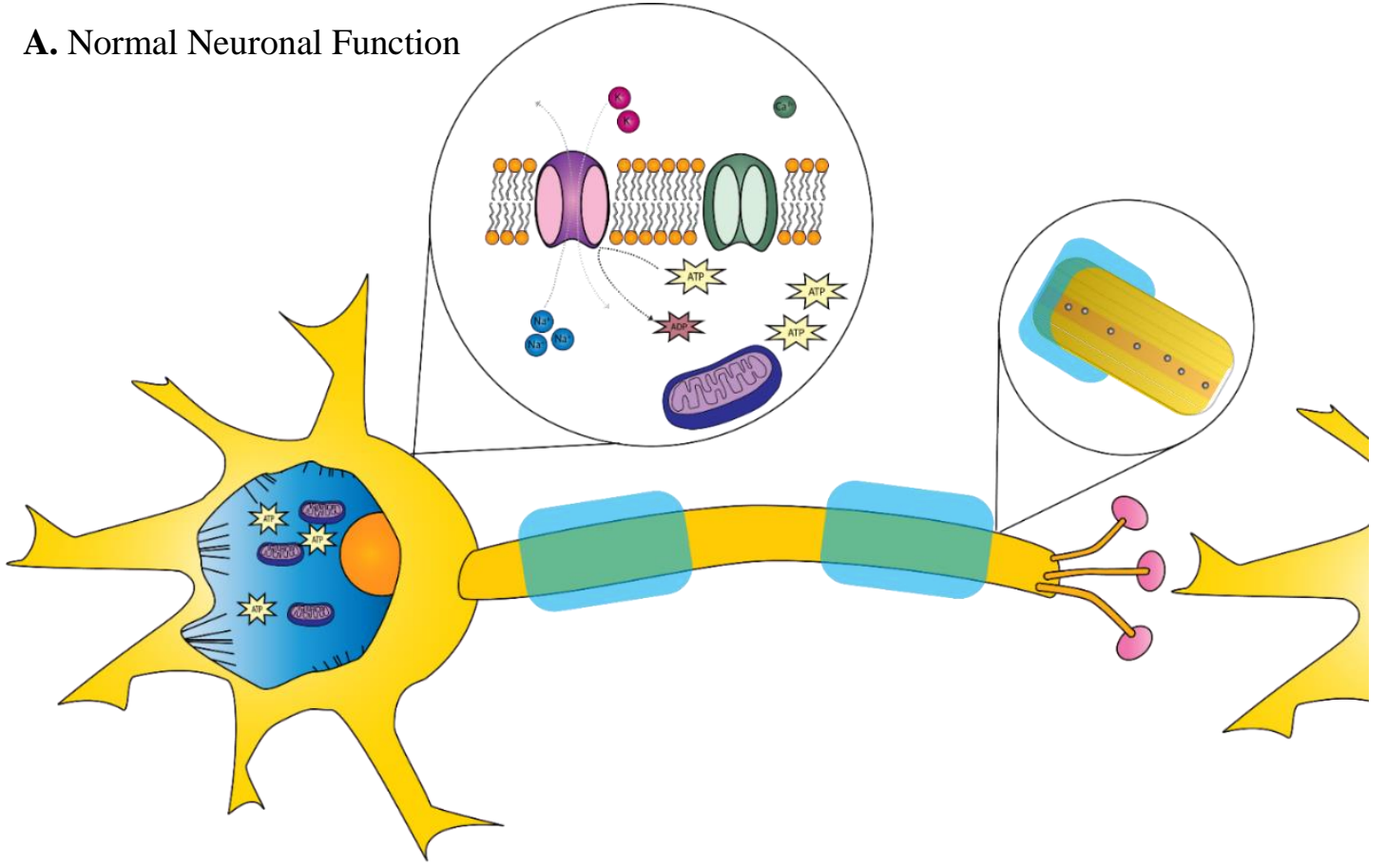

B. Post-Concussion/mTBI Neurometabolic Cascade

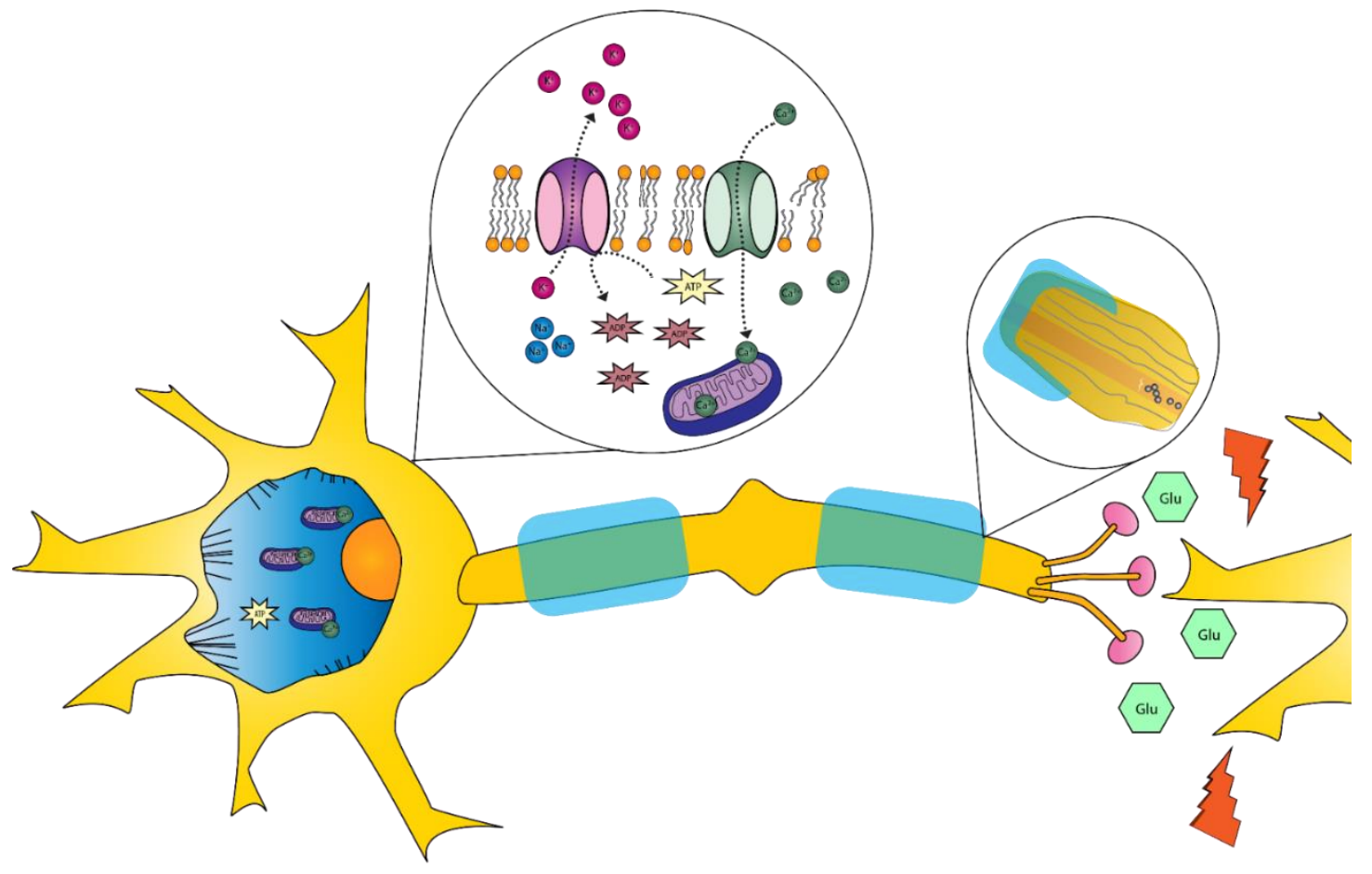




\subsection{Concussion Epidemiology}

\subsubsection{General Population}

Each year 1.6-3.8 million concussions occur from sports/recreation (Langlois, RutlandBrown, \& Wald, 2006). Of all TBI severities, concussion composes 70-90\% of injuries (Cassidy et al., 2004). The estimated concussion hospitalization rate in the United States ranges from 100300 per 100,000 individuals (Cassidy et al., 2004). A survey of United States adults in 1991 found that $25 \%$ of individuals who reported loss of consciousness did not seek medical care (Sosin, Sniezek, \& Thurman, 1996). Therefore, rates accounting for underreporting estimate 600 individuals per 100,000 sustain a concussion annually (Cassidy et al., 2004; Langlois et al., 2006). Most recently, a study of concussion presenting to the emergency department in 2012 observed 807.9 individuals with concussion per 100,000 (Cancelliere, Coronado, Taylor, \& Xu, 2017). This is a significant increase from the early 2000s, where the rate was 503.1-569.4 per 100,000 (Bazarian et al., 2005; Cancelliere et al., 2017). CDC reports of emergency department visits show that for all age groups concussion has increased since 2001 (Centers for Disease Control and Prevention, 2016c)(Figure 2.4.1). However, these are also likely underestimated since there is a poor diagnosis agreement across clinicians for concussion cases (Ryu, Feinstein, Colantonio, Streiner, \& Dawson, 2009). When an expert clinical reviewer examined 876 records for potential concussion, the expert reviewer agreed with the diagnosis in only 516 cases (Ryu et al., 2009). Of these cases, 124 the original physician did not diagnosis a concussion but the expert reviewer did (Ryu et al., 2009). Additionally, each of the above studies depended on hospitalization codes and records to identify concussion cases. Unfortunately, ICD-9 codes are correctly applied to concussion cases $46 \%$ of the time (Bazarian, Veazie, Mookerjee, \& Lerner, 2006). Another study found that $56 \%$ of concussion cases in the ED did not have their concussion documented in their medical record (Powell, Ferraro, Dikmen, Temkin, \& Bell, 2008). The high rate of underreporting and misdiagnosis likely leads to underestimates of the true concussion burden. 
Figure 2.4.1. Concussion Prevalence by Age and Year

\section{Mild TBI Rate Estimate by Age and Year}

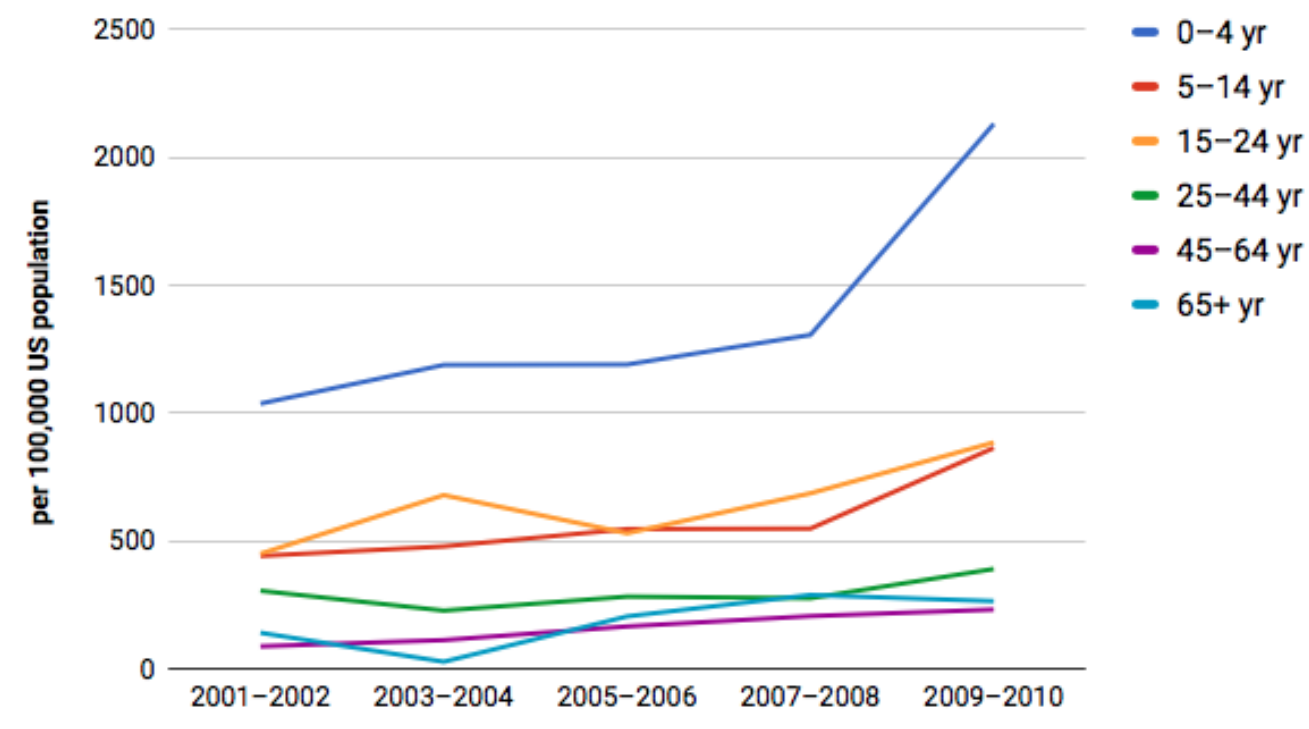

Note: Adapted from CDC 2016 (Centers for Disease Control and Prevention, 2016c)

Concussion occurs differentially across sex, age, socioeconomic groups, and race. Males are twice as likely to sustain a concussion (Bazarian et al., 2005; Cassidy et al., 2004; Voss, Connolly, Schwab, \& Scher, 2015). Mild TBI is most common among those under five years old (Bazarian et al., 2005; Voss et al., 2015). Those 5-14 and 15-24 years old have the next highest concussion rates (Bazarian et al., 2005; Centers for Disease Control and Prevention, 2016b). Among males, low socioeconomic status was associated with increased risk for concussion (Nordstrom, Edin, Lindstrom, \& Nordstrom, 2013). However, this study was limited to males conscripted for military service in Sweden, and it is unknown whether this increased risk persists across all sex and age groups. Finally, American Indian/Alaska Natives have the highest concussion rate but is not significantly different than white, African American, or Asian/Native Hawaiian/Pacific Islanders (Bazarian et al., 2005). Asian/Native Hawaiian/Pacific Islanders have significantly lower rates than white or African American cohorts. There was no significant difference between white and African American concussion rates (Bazarian et al., 2005).

Among the general population, the most common mechanisms of injury are falls and motor vehicle accidents (Bazarian et al., 2005; Cassidy et al., 2004). Falls accounted for 44\% of all concussions (Cancelliere et al., 2017). Motor vehicle accidents account for $25-37 \%$ of concussions (Annegers, Grabow, Kurland, \& Laws, 1980; Fife, 1987). However, it is important 
to consider age when evaluating mechanism. Among young children and the elderly, falls constitute over 70\% of TBIs (Bazarian et al., 2005; Centers for Disease Control and Prevention, 2016a). However, among 15-24 year-olds, motor vehicle accidents (21\%), assaults(22\%), and falls $(21 \%)$ equally contribute to TBI-related emergency department visits (Centers for Disease Control and Prevention, 2016a)

\subsubsection{Athletes}

By the end of a collegiate athletic career, $50 \%$ of athletes have sustained a concussion (Llewellyn, Burdette, Joyner, \& Buckley, 2014). Of these injuries, the majority are diagnosed (34\%), but a significant proportion are not diagnosed by the clinician (undiagnosed, 26\%), or not reported by the athlete (unreported, 11\%) (Llewellyn et al., 2014). The risk for concussion exists in all sports, regardless of contact level (Hainline, 2016) and while recent rule changes and education have attempted to make sports safer, concussion risk cannot be completely eliminated.

Between the 1988-1989 and 2014-2015 seasons, the concussion rate increased $247 \%$ at the collegiate level (Gessel, Fields, Collins, Dick, \& Comstock, 2007; Hootman et al., 2007; Kerr et al., 2017; Zuckerman et al., 2015) (Figure 2.4.2). Between 1988-1989 and 2004 the average annual increase was 7\% (Hootman et al., 2007). Compared to anterior cruciate ligament injuries, which increased $1.6 \%$ per year, concussions occur 1.5 to 2.5 times more often than ACL injuries (Hootman et al., 2007) (Figure 2.4.2). More recently, the concussion rate has plateaued from 5.49 per 10,000 AEs (Zuckerman et al., 2015) to 5.54 per 10,000 AEs (Kerr et al., 2017) across the same sports. While concussion diagnosis and reporting frequency may have increased over

the last twenty years, increased knowledge, education, reporting, and medical attention are likely causes for the increased concussion rate (Daneshvar, Nowinski, McKee, \& Cantu, 2011). Thus, while concussions may be occurring at a higher frequency than twenty years ago it is thought that increased knowledge, awareness, and reporting are the cause of the substantial increase. For example, concussion rates across three Division I football teams doubled after the NCAA implemented a concussion management program in 2010 (Kilcoyne et al., 2014). Rates increased from 0.54 per 1000 AEs to 1.16 per 1000 AEs in the 2010-2011 season (Kilcoyne et al., 2014). As part of these guidelines, all student-athletes were educated on the signs and symptoms of concussion and signed a statement that they will report all concussion signs and symptoms to the medical staff. Additional changes included baseline and post-injury cognitive assessments, any athlete diagnosed with a concussion will be removed from sport for a minimum of 1 day, and 
physician will determine return to play (Kilcoyne et al., 2014). Another study examining the role of legislation on high school and collegiate athlete concussion reporting found that while concussion rates in 2013 were comparable to 1999-2002, athletes were more likely to report their concussion (71\% versus 47\%) (LaRoche et al., 2016). These studies demonstrate that while concussion rates have increased the past decade, the underlying true concussion frequency has likely remained similar. New guidelines and legislation increase the capture rate, yielding a more accurate estimate of the true concussion rate.

Figure 2.4.2. Collegiate Concussion and ACL Injury Rates over Time 7

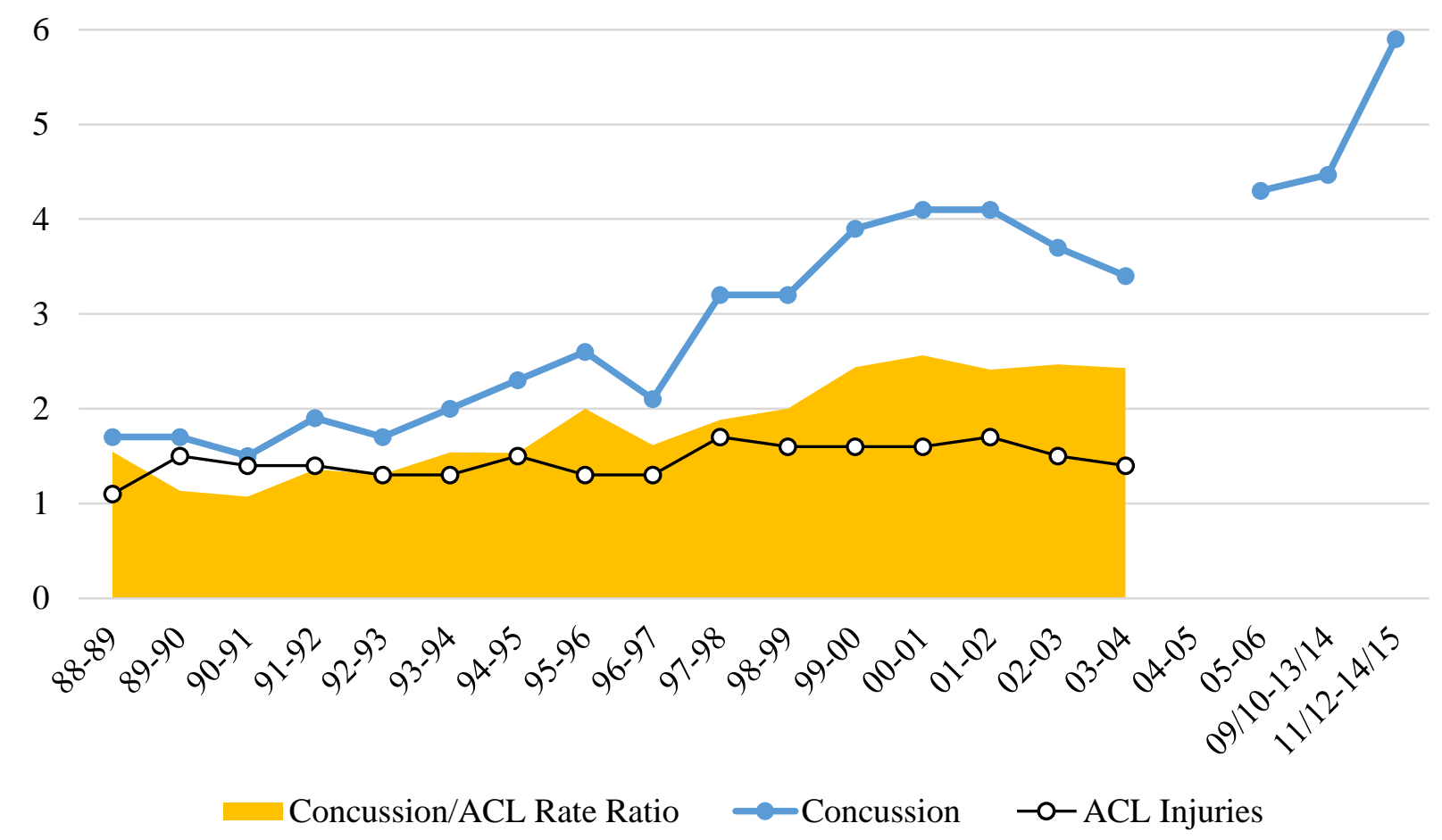

Note: Adapted from Hootman et. al. 2007 (Hootman et al., 2007); Zuckerman et. al. 2015 (Zuckerman et al., 2015); Kerr et. al. 2017 (Kerr et al., 2017)

Between 1988 and 2004, ice hockey (men's and women's), women's soccer, and football had the highest concussion rates all above 4 per 10,000 AEs (Hootman et al., 2007) (Table 2.4.1). Over time, wrestling, experienced the largest increase in concussion rate from 2.5 (Hootman et al., 2007) to a high of 10.92 per 10,000 AEs by 2014 (Zuckerman et al., 2015) . The latest estimates rank wrestling, women's ice hockey, football, men's ice hockey, and men's and women's soccer as the highest concussion rate sports (Kerr et al., 2017) (Table 2.4.1). However, 
non-contact sport athletes also experience concussions. Tennis, swim/dive, outdoor track, indoor track, and women's cross country all experience concussions (Zuckerman et al., 2015). The only sport that did not experience a sport-related concussion between 2009 and 2014 was men's cross country (Zuckerman et al., 2015).

Table 2.4.1. Concussion Rates (per 10,000 AE) and 95\% Confidence Intervals by Sport and Year

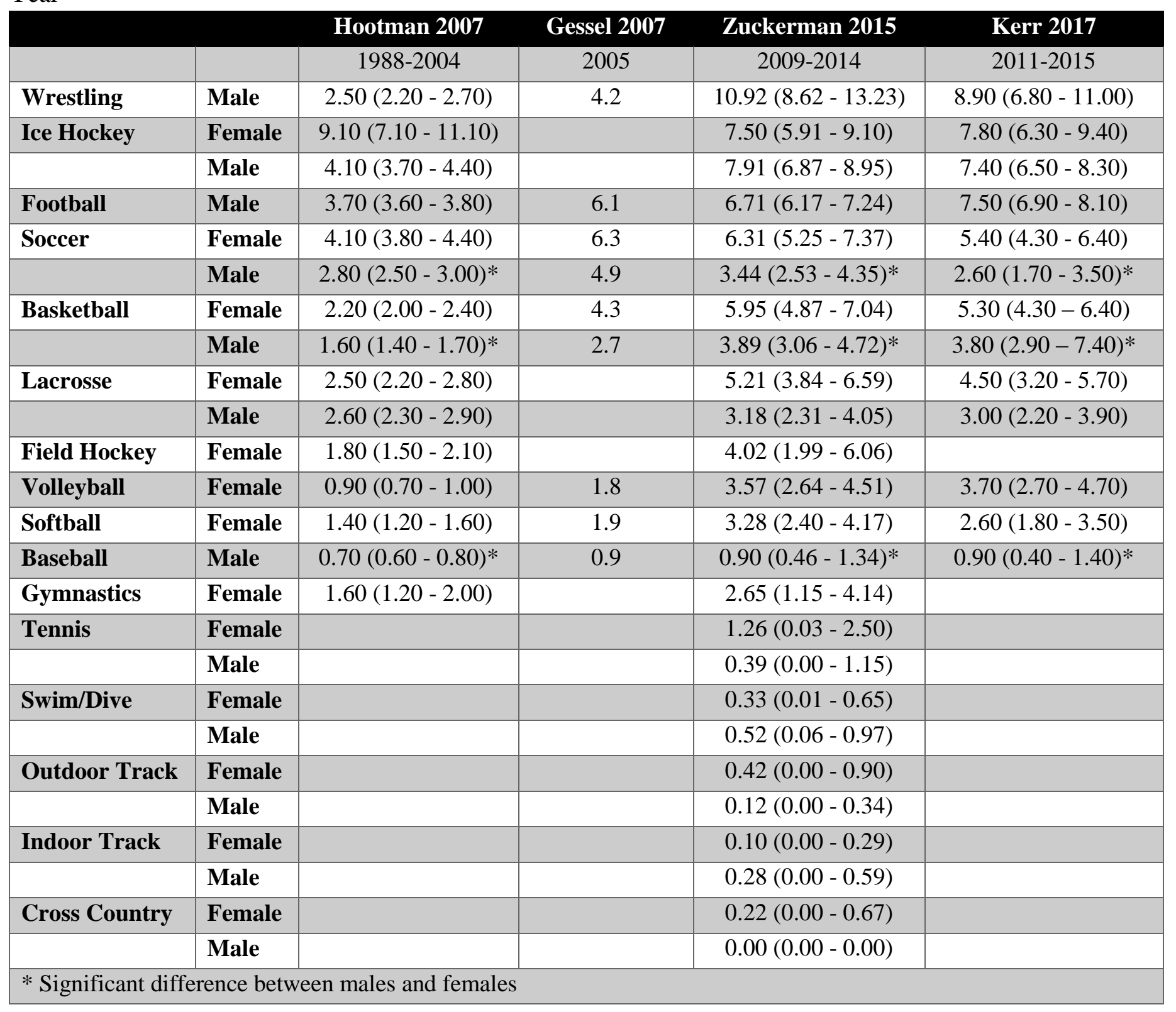


While the rates are higher for wrestling and ice hockey compared to football, the number of concussions for football are greater due to more participants. The average number of concussions for a football team per year is $5.63 \pm 5.36$, higher than wrestling $2.34 \pm 3.38$, women's ice hockey $1.69 \pm 1.90$, and men's ice hockey $2.05 \pm 2.11$ (Kerr et al., 2017). When examining team risk, the proportion of teams per season with at least one concussion, men's ice hockey poses the greatest risk with $92 \%$ of teams having a concussion per season. Football teams have a 79\% risk followed by women's soccer 54\% (Kerr et al., 2017). At the individual level, a wrestler has a $7.9 \%$ risk of sustaining a concussion in a given season, followed by women's ice hockey (7.2\%), men's ice hockey (6.9\%), and football (5.3\%) (Kerr et al., 2017).

Across sex-comparable sports, females report more concussions than males (Hootman et al., 2007; Kerr et al., 2017; Zuckerman et al., 2015). The most consistent sports with a significant sex effect were soccer $(R R=1.83-2.10)$, basketball $(R R=1.42-1.53)$, and baseball/softball $(R R$ = 3.01-3.65) (Kerr et al., 2017; Zuckerman et al., 2015). Within ice hockey and lacrosse (Kerr et al., 2017) there was no significant sex effect. Ice hockey and lacrosse, played by both sexes, has the greatest differences in rules between the male and female versions of the sport. Both men's ice hockey and lacrosse are a full-contact sport where body contact is legal. Comparatively, body checking is illegal in women's ice hockey. Despite major rule differences, both males and females sustain concussions at a similar rate in ice hockey and lacrosse. Most concussions in ice hockey are due to player-to-player contact for both men (73.2\%) and women (49.4\%) (Zuckerman et al., 2015). Thus, the incidental contact and typical gameplay rather than body checking are likely causes of high concussion rates in the men's and women's sports. While females have a higher rate of concussions than males, this difference is limited to soccer, basketball, and baseball/softball.

The higher concussion rate observed among females has been attributed to biological and psychological factors. Reduced neck strength relative to male peers has been hypothesized as a possible biomechanical mechanism for concussion risk. With less neck strength female athletes are less equipped to brace for an oncoming impact and are less able to reduce accelerations transmitted to the brain (Collins et al., 2014; Tierney et al., 2005). While the luteal phase of the menstrual cycle has been associated with longer recovery time after concussion (Bazarian, Blyth, Mookerjee, He, \& McDermott, 2010), there has been no link established between hormones, 
menstrual cycle and concussion risk. Further research is needed to better understand the possible biological drivers of concussion risk differences across males and females.

Beyond fundamental biological differences, females are also psychologically different than males. Even outside of concussion injuries, females report more symptoms and to a greater severity than males (Cameron 2017; CARE Meeting). After a concussion females report seven more symptoms than males (Covassin, Elbin, Bleecker, Lipchik, \& Kontos, 2013). The increased symptoms reporting may contribute to females being more honest or feeling less pressure to continue to play when injured compared to males (Dick, 2009).

\subsubsection{Service Members}

Since 2000, 297,478 service members have been diagnosed with a concussion (Defense and Veterans Brain Injury Center, 2017). The concussion rate significantly increased between 2000 and 2006 from 3.4 per 10,000 to 7.0 per 10,000 service members (Ivins \& Ivins, 2010). The dramatic increase was due to the military conflicts, Operation Enduring Freedom/Operation Iraqi Freedom (OEF/OIF). However, many estimates rely on ICD-9 codes which can misclassify concussion (Bazarian et al., 2006). Also, when a service member sustains multiple TBIs, the most severe TBI diagnosis is recorded (Defense and Veterans Brain Injury Center, 2017). Thus, current reports likely underestimate the current concussion burden among service members.

While increasing concussion rates during OEF/OIF, appear to indicate greater combatrelated injuries, $80 \%$ of concussions occurred outside of combat and within the continental United States (Cameron et al., 2012). Therefore, the majority of concussions may be sustained by similar mechanisms observed in civilians (Centers for Disease Control and Prevention, 2016a). Analyses of combat-related concussion reveal that those with concussion tended to be younger, more junior rank, and more likely to be male (Hoge et al., 2008). While males appear to be more likely to sustain a concussion during combat, females, if studied, composed less than $2 \%$ of each

of the study samples (Hoge et al., 2008; Wilk et al., 2010). The study by Cameron and colleagues had a sample that was $12 \%$ female. Similar findings were observed by Cameron and colleagues, whereby males had a $10 \%$ increased risk of sustaining a concussion compared to females, that younger, and more junior ranked service members were at greater risk (Cameron et al., 2012). Despite consistent sex findings, it is important to note that each study was completed before all combat jobs were opened to women in April 2015. 
Compared to active duty service members there has been relatively little research on concussion incidence and patterns among service academy cadets. Unlike their civilian counterparts, all cadets at the service academy must participate in a sport. The sport could be at the varsity level, intercollegiate club, or intramural level. Two studies investigated concussion rates within football and men's and women's rugby (Kilcoyne et al., 2014; Peck, Johnston, Owens, \& Cameron, 2013). In the 2010-2011 season, the concussion rate across three service academy football teams was 11.3 per 10,000 AEs (Kilcoyne et al., 2014). This rate is greater than the 6.71-7.50 per 10,000 AEs (Kerr et al., 2017; Zuckerman et al., 2015) rate reported by the NCAA ISP. The difference in rates could be that the Kilcoyne (Kilcoyne et al., 2014) study was limited to three Division I sites while the NCAA ISP study collects data from over 1000 NCAA institutions and across all three divisions.

Another study of rugby at a single service academy found that males $(0.33$ per 10,000 AEs) and females (0.44 per 10,000 AEs) sustain concussions at the same rate(Peck et al., 2013). This result is similar to the findings by Zuckerman (Zuckerman et al., 2015) and Kerr (Kerr et al., 2017), that contact sports like ice hockey and lacrosse do not demonstrate a significant effect of sex. Rugby rules are the same for men and women (Peck et al., 2013). Given the high level of contact for each sex, similar concussion rates are observed.

\subsubsection{Summary}

Previous epidemiological studies of concussion/concussion have focused on sport-related injuries or combat-related injuries. While these studies highlight populations and subpopulations at greatest risk for concussion they do not account for injuries occurring outside of sports or combat. Similarly, the above studies of hospitalization data may underestimate the number or sport or recreation-related injuries as many would be diagnosed and treated by the team's medical staff and thus never present to the hospital(Broglio et al., 2014). Additionally, there is little research on concussion incidence among service academy cadets. To understand the true incidence and effects of concussion among service academy cadets research must obtain data on all concussions regardless of mechanism. Since cadets may be at increased concussion risk due to sport participation and academy training, it will be important to parse out at-risk groups and injury patterns to improve cadet safety. 


\subsection{Concussion Recovery}

While concussion is a prevalent injury (Langlois et al., 2006), most individual have a progressive recovery without the need for medical intervention (McCrory et al., 2017). However, a subset of individuals have persistent symptoms after a concussion (McCrea et al., 2013; Nelson et al., 2018; Nelson et al., 2016). Characterizing typical recovery trajectories and factors related to recovery is critical as during recovery the brain is susceptible to secondary injury (Guskiewicz et al., 2003; Prins, Alexander, Giza, \& Hovda, 2013) and increasing concussions have been associated with cognitive impairment (Guskiewicz et al., 2005) and depression (Kerr et al., 2012) later in life.

After a concussion, there is an immediate symptomatic period where the individual may experience increased symptoms, and impaired cognition and postural stability (McCrea et al., 2003). Once symptoms resolve, a return to activity (RTA) protocol is initiated (McCrory et al., 2017) whereby exercise is introduced and increasing in exertion as long as symptoms do not reemerge. Thus, the total time loss from activity is the number of days between injury and when the RTA protocol is completed. Previous concussion recovery studies have either investigated the symptom duration (Guskiewicz et al., 2003; Makdissi et al., 2010; Marshall, Guskiewicz, Shankar, McCrea, \& Cantu, 2015; McCrea et al., 2013; McCrea et al., 2003; Nelson et al., 2018; Nelson et al., 2016; Teel, Marshall, Shankar, McCrea, \& Guskiewicz, 2017) or total time loss (Covassin, Moran, \& Elbin, 2016; Davis-Hayes et al., 2017; Kennedy et al., 2012; Lau, Lovell, Collins, \& Pardini, 2009; Lau, Kontos, Collins, Mucha, \& Lovell, 2011). However, symptom duration, RTA protocol duration, and total time loss are likely influenced by different factors. The pathophysiological response to concussion creates the cognitive, somatic, and emotional symptoms along with impaired cognition and postural stability (Giza \& Hovda, 2014). Thus, the symptom resolution period is likely influenced by underlying brain physiology. Currently, clinical measures of symptoms, cognition, and neurological function are used by a clinician to determine when an individual is recovered (Nelson, Janecek, \& McCrea, 2013). Consequently, a portion of symptom duration is also influenced by clinical management and decision making (Figure 2.5.1). Meanwhile, RTA guidelines (Doolan, Day, Maerlender, Goforth, \& Gunnar Brolinson, 2012) guiding clinical management likely have a stronger influence on RTA protocol duration than underlying brain physiology. To test these assumptions research should evaluate 
which individual, injury, and environmental characteristics influence each portion of total time loss.

Figure 2.5.1. Components of Concussion Recovery

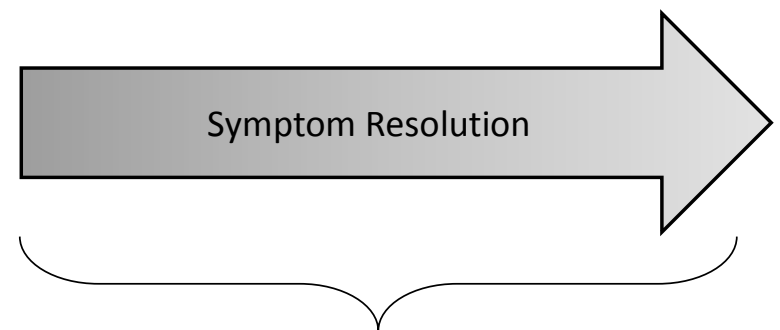

Duration until Asymptomatic

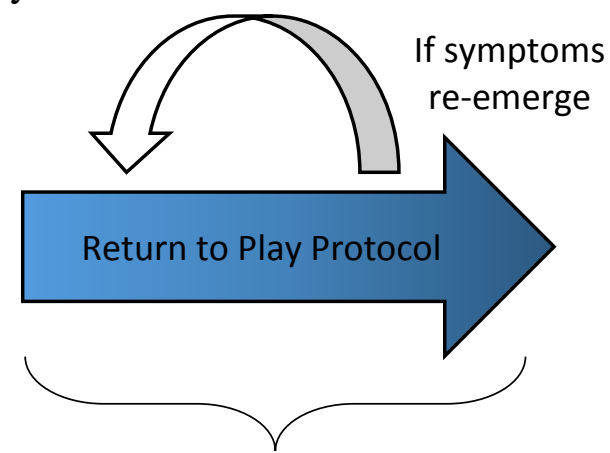

Return to Play Protocol Duration

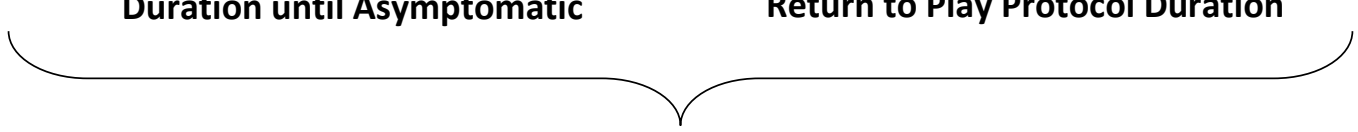

Return to

Play

Total Time Loss

\subsubsection{Factors Associated with Concussion Symptom Duration}

After a concussion there is an acute increase in symptom prevalence and severity (Chin et al., 2016; McCrea et al., 2003). One of the first studies to compare symptom scores overtime after a concussion compared concussed collegiate football players to match non-concussed peers (McCrea et al., 2003). Total symptom scores increased after a concussion but were similar to controls by post-injury day five (McCrea et al., 2003). However, since this study only examined male collegiate football players it was unclear whether symptom duration was typical among other athletes and female populations. More recently, Chin and colleagues (Chin et al., 2016) examined symptom scores of high school and collegiate athletes. While the sample was predominately male (80\%), athletes participated in a variety of sports. Compared to controls, symptom scores were significantly higher among concussed athletes until day eight post-injury (Chin et al., 2016). While symptom duration varied slightly across studies, the differences may be attributable to different study samples. Younger athletes (Williams, Puetz, Giza, \& Broglio, 2015) and females (Zuckerman et al., 2014) have been shown to have longer symptom duration. Thus, including high school athletes and females may be a more representative reflection of true symptom recovery. Overall, most athletes appear to recover within ten days. 
Pre-concussion individual characteristics and acute concussion characteristics are hypothesized to influence recovery duration (Iverson et al., 2017). Pre-injury characteristics consist of demographic and pre-morbid clinical risk factors. There is mixed evidence for the effect of female sex, prior concussions, psychological disorders, and headache on symptom duration (Iverson et al., 2017). Studies finding evidence that females took longer to recover more often included youth (Eisenberg, Andrea, Meehan, \& Mannix, 2013; Zuckerman et al., 2014) or findings were limited to specific sex-comparable sports (Wasserman, Kerr, Zuckerman, \& Covassin, 2016). Additionally, these studies used only univariate models to assess the relationship between sex and recovery. Studies that used multivariate models to assess for other factors related to recovery found that sex was not a significant predictor of symptom resolution time (Nelson et al., 2018; Nelson et al., 2016).

Similarly, studies assessing the previous concussion have found a positive association between previous concussion and symptom duration (Guskiewicz et al., 2003; Wasserman et al., 2016) while other investigations have not yielded significant results (McCrea et al., 2013; Teel et al., 2017). Since multivariate models of symptom duration did not find a significant effect of previous concussion (McCrea et al., 2013), we expect the role of previous concussion to have a limited impact on symptom duration when accounting for multiple individual and injury characteristics.

Post-injury characteristics, including loss of consciousness (LOC), post-traumatic amnesia (PTA), retrograde amnesia (RGA), greater symptom number or severity, and specific symptoms (e.g. headache, dizziness, balance) have also been identified as recovery factors (Iverson et al., 2017). Acute injury characteristics including LOC, PTA, and acute symptom burden have been associated with recovery duration. LOC and PTA have been associated with increased symptom duration (McCrea et al., 2013), but in more recent studies have found no effect (Nelson et al., 2016). Earlier concussion studies had larger proportions of athletes with observable concussion signs (e.g. LOC). Fewer concussions associated with observable signs, and thus smaller sample sizes, may be responsible for conflicting results.

Acute concussion symptoms have had a more consistent association with symptom duration (Iverson et al., 2017). Specifically, increased symptom severity(McCrea et al., 2013) or number (Nelson et al., 2016) increases the likelihood of prolonged symptom duration. However, both of these studies were comprised of an $80 \%$ male sample and only included sports-related 
concussions. Thus, it is unknown whether total symptom number or severity has the same influence on recovery among females and non-sport related concussions. Future studies should address the role of acute symptom burden among females and non-sport related concussions.

While greater acute symptom number and severity appears to prolong symptom duration (McCrea et al., 2013; Nelson et al., 2016), few studies have investigated which specific symptoms influence recovery. Concussion symptoms are non-specific and are also reported by individuals without a concussion (Asken, Snyder, et al., 2017; Chin et al., 2016). Headache, difficulty concentrating, drowsiness, trouble falling asleep, irritability, and difficulty remembering are the most commonly reported symptoms at baseline (Asken, Snyder, et al., 2017) (Figure 2.5.2A). Comparatively, dizziness, sensitivity to light, balance problems, visual disturbance, and disorientation are rarely endorsed at baseline, but their prevalence increases over 20\% post-concussion (Asken, Snyder, et al., 2017; Wasserman et al., 2016)(Figure 2.5.2). Consequently, we hypothesize that these less commonly reported baseline symptoms are more specific to a concussion and provide greater information on subsequent recovery. 
Figure 2.5.2. Comparison of Baseline and Post-Concussion Symptom Prevalence

A.

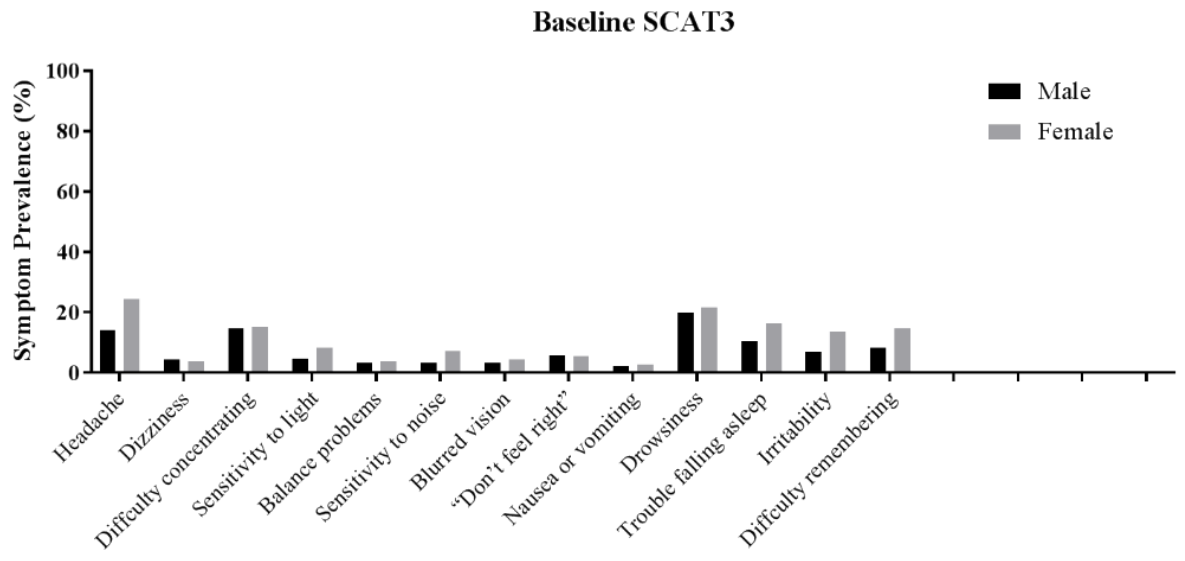

B.

Post-Concussion Symptoms

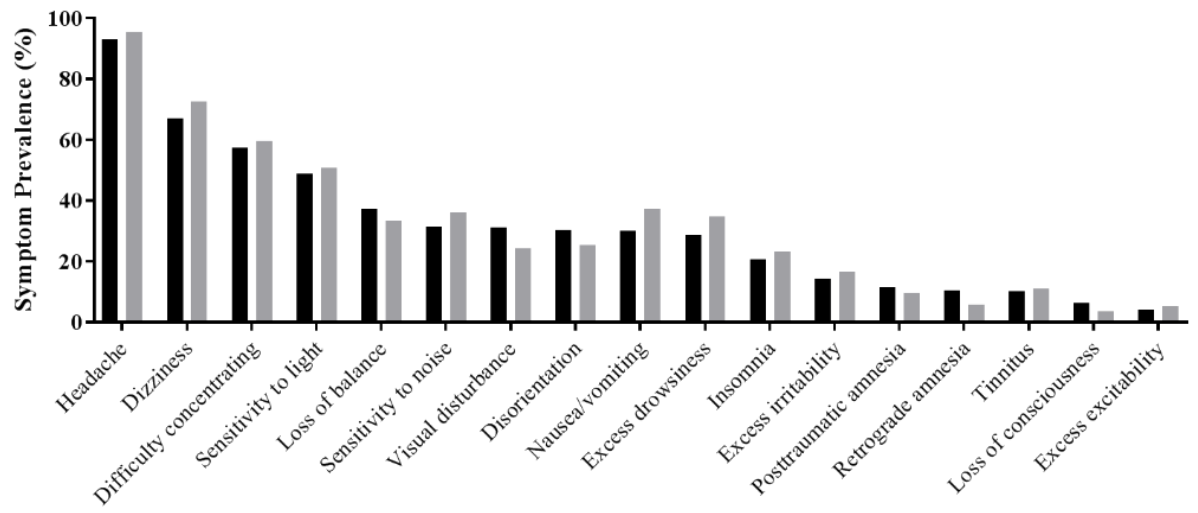

C.

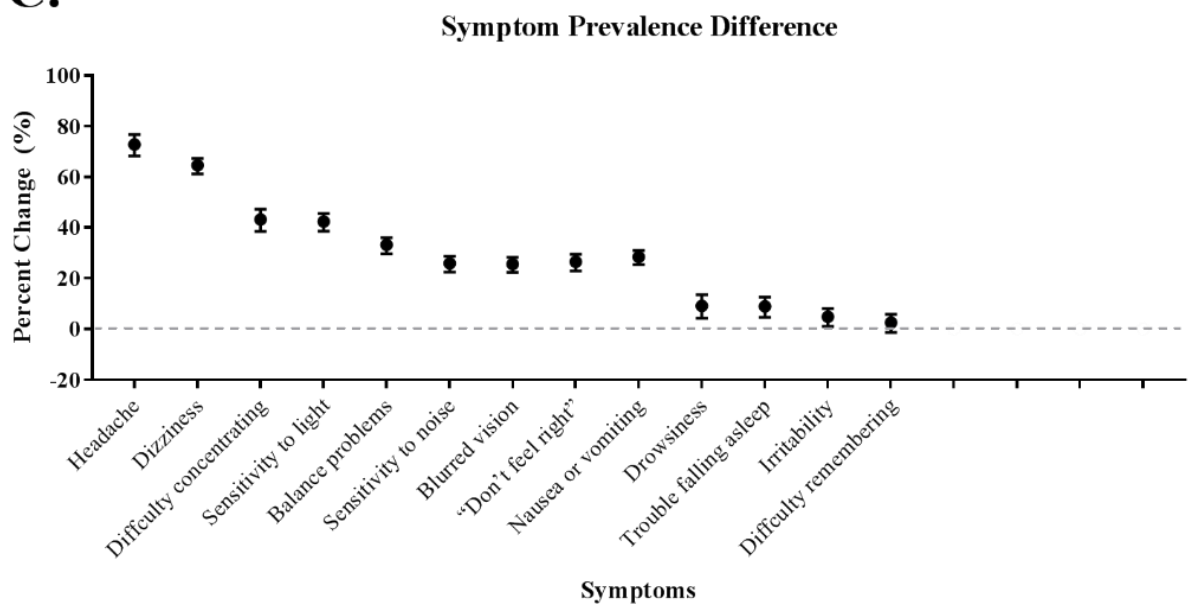

Figure 2.5.2A adapted from Asken et. al. 2016. Figure 2.5.2B adapted from Wasserman et. al. 2016.

Figure 2.5.2C compares percent change in symptoms from Asken et. al. 2016 and Wasserman et. al. 2016 


\subsubsection{Factors Associated with Total Time Loss after Concussion}

Similarly, the total number of symptoms is associated with longer total time loss (DavisHayes et al., 2017; Lau et al., 2011; Makdissi et al., 2010). Among service members, acute symptoms also predicts total time loss (Kennedy et al., 2012). An initial study of high school football athletes identified increased migraine, cognitive, and sleep symptom clusters to be associated with greater time loss (Lau et al., 2009). Further examination of the symptom clusters found that vomiting, dizziness, difficulty concentrating, and nausea symptoms had the largest effects between prolonged and normal time loss. In a follow-up study seeking to identify individual symptom predictors of total time loss, dizziness was the only significant symptom associated with total time loss (Lau et al., 2011). This multivariate analysis revealed that selfreported dizziness on the sideline was associated with six-fold increased odds for total time lost more than 21 days (Lau et al., 2011). While preliminary evidence exists for individual symptoms

predicting recovery duration, this study was conducted on male high school football players (Lau et al., 2011). Thus, it is unknown whether there is a similar effect of dizziness and more symptoms among other athletes, females, or non-sport related concussions.

One previous study found no effect of sex on total time loss within sex-comparable sports (Wasserman et al., 2016). Comparatively, an earlier study found a significant effect of sex, within soccer and basketball athletes, with females having greater total time loss than males (Covassin et al., 2016). However, this effect was limited to practice sessions only. There was no difference in mean total time loss between sexes when the concussion occurred during competition (Covassin et al., 2016). Consequently, it is possible that rather than a sex effect, there is an effect of athletic trainer presence and immediate reporting. If athletic trainers are not present at female practice sessions, it is possible concussions would go unreported or unrecognized. In a study of high school athletes, those without an athletic trainer at their school were five times more likely not to report a concussion because they did not know they had a concussion (Wallace, Covassin, Nogle, Gould, \& Kovan, 2017). If the concussion was unreported or unidentified during practice, the female athlete was unlikely to be immediately removed from practice. Immediate removal has been associated with shorter total time loss due to concussion (Asken et al., 2016). Consequently, there is likely other competing factors influencing total time loss, rather than sex. 
Immediate removal has been shown to be a predictor of total time loss among collegiate (Asken et al., 2016) and adolescent athletes (Elbin et al., 2016). Among youth athletes, there was an association between on-field signs or symptoms and whether the athlete was immediately removed (Elbin et al., 2016). However, the limited sample size and rarity of LOC or amnesia may have limited the researchers' ability to assess for these confounders. Among collegiate athletes, there was no association between immediate removal and sex, prior concussion, or event type (game versus practice) (Asken et al., 2016). While analyses controlled for acute symptom severity, the relationship between acute symptoms and immediate reporting was not assessed. Thus, it is unknown whether acute signs (e.g. LOC, amnesia) and symptoms are associated with immediate removal. We hypothesize that greater signs and symptoms would help clinicians more easily identify an athlete with a possible concussion and thus remove them from play. Consequently, immediate removal from play might be a surrogate measure of acute signs and symptoms.

Prior concussions have also been hypothesized to influence recovery (Iverson et al., 2017). Recurrent concussions have been shown to prolong recovery and exacerbate the injury response if the injuries occur close enough in time (Prins, Hales, Reger, Giza, \& Hovda, 2010; Vagnozzi et al., 2008). Among collegiate athletes, recurrent concussions occurring within the same season have been associated with greater total time loss (Wasserman et al., 2016). However, in a study controlling for other individual injury characteristics, no effect of previous concussion was observed (Asken et al., 2016). Similar to sex, previous concussion may have a univariate effect on total time loss. However, other factors have stronger predictive ability, and thus, prior concussion is unlikely to strongly influence total time loss when accounting for other individual and injury characteristics.

\subsubsection{Summary}

Recovery after a concussion is composed of two elements: symptom duration and return to activity protocol. While acute symptoms have the most consistent effect on symptom duration and total time loss, it is unknown whether acute symptoms also influence return to activity protocol duration. Moreover, most previous studies have examined univariate factors without controlling or examining for multivariate effects. A summary of the current factors related to recovery are described in Figure 2.5.3. Future research should investigate multiple factors effects 
on symptom and return to activity protocol durations. Also, elucidating individual symptoms associated with recovery may identify points of clinical intervention to improve recovery time.

Figure 2.5.3. Summary of Recovery-Related Factors

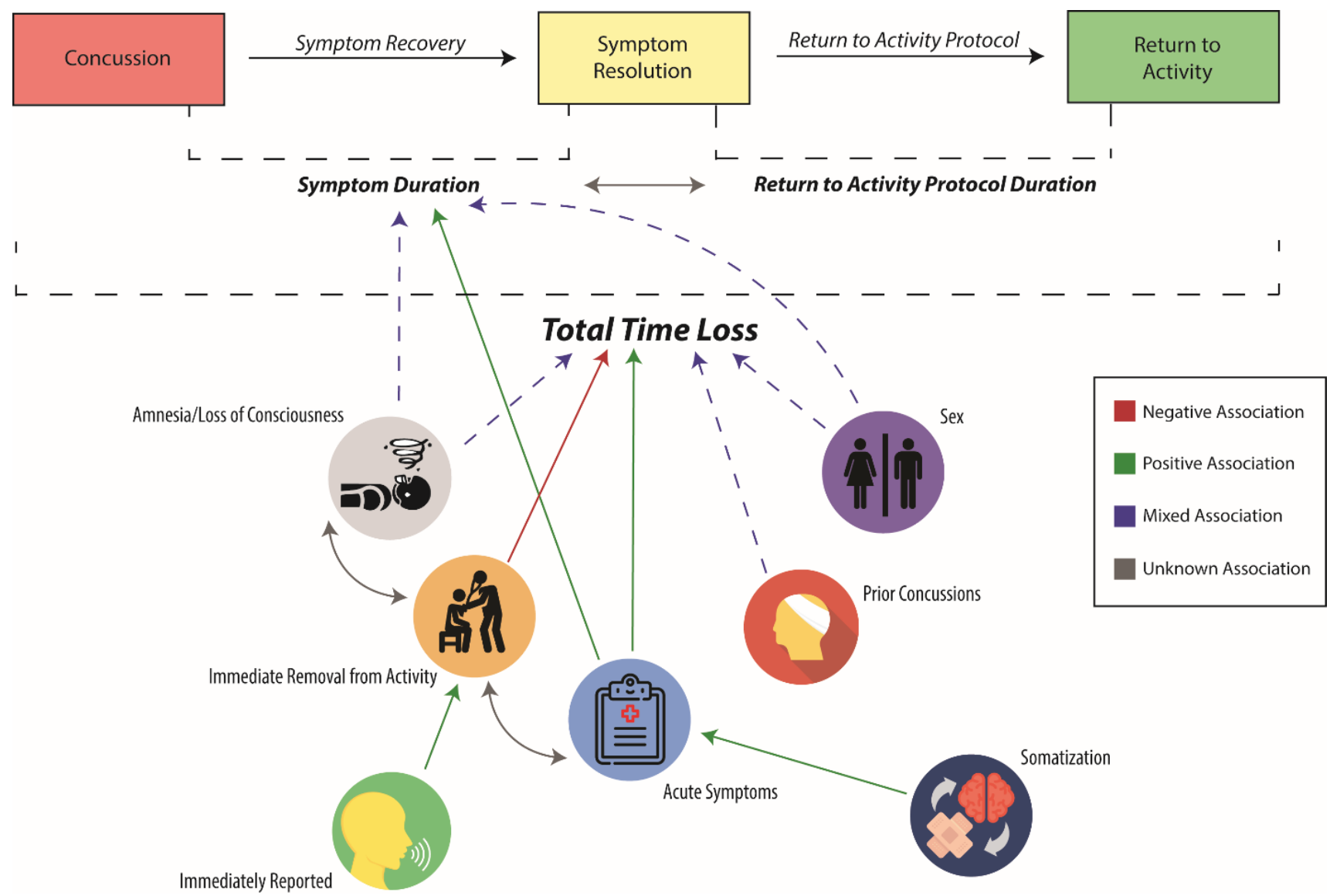

\subsection{The Association between Concussion and Mental Health}

2.6.1 Psychological Symptoms Associate with Injury Risk

Psychological symptoms have been associated with sports injury (Junge, 2000; Li, Moreland, Peek-Asa, \& Yang, 2017). Most recently, Li and colleagues (Li et al., 2017) investigated preseason anxiety and depression scores and injury risk within collegiate athletes. 
Injuries included musculoskeletal injuries and concussion with concussions accounted for $9 \%$ of the injuries collected ( $\mathrm{Li}$ et al., 2017). Athletes with anxiety symptoms experienced 2.3 times increased subsequent injury risk compared athletes without preseason anxiety symptoms ( $\mathrm{Li}$ et al., 2017). Depression symptoms alone were not associated with increased injury risk. However, males who experienced co-occurring anxiety and depression symptoms during preseason were more injury prone than male athletes without any symptoms. While not specific to concussion, anxiety and depression are associated with increased injury risk among collegiate athletes ( $\mathrm{Li}$ et al., 2017). While mood and anxiety disorders have been suggested as possible risk factors, no study had directly assessed whether preexisting disorders increase concussion risk.

While no study has investigated whether preexisting mood and anxiety disorders increase concussion risk, potential injury risk mechanisms are outlined below. Depression and anxiety each effect cognitive and motor control increasing the likelihood of an injurious situation. Additionally, brain vulnerabilities may specifically increase concussion likelihood.

Depression and anxiety each effect the executive function system. Patients with major depressive disorder perform significantly worse on working memory tasks (Doumas, Smolders, Brunfaut, Bouckaert, \& Krampe, 2012), have difficulty sustaining motivation (Marazziti, Consoli, Picchetti, Carlini, \& Faravelli, 2010), and are susceptible to fatigue (Marazziti et al., 2010) when compared to healthy controls. Moreover, executive function deficits persist even among remitted depressed patients (Nakano et al., 2008; Paelecke-Habermann, Pohl, \& Leplow, 2005). During periods of increased anxiety, an individual's focus is drawn to threat-related stimuli, limiting their concentration and information processing (Eysenck, Derakshan, Santos, \& Calvo, 2007). Drawing attention away from task-relevant stimuli impairs movement execution, resulting in impaired performance. For example, soccer players under stressful conditions have less accurate goal kicks (Wilson, Wood, \& Vine, 2009). Additionally, anxiety may cause individuals to focus their attention internally rather than externally. This internal focus is particularly problematic for expert performers as it impairs the automaticity of well-learned movement and thus decreases performance (Beilock \& Carr, 2001).

Depression and anxiety impairs a variety of executive functioning domains. Motor control, volition, fatigue, and attention impairment can increase injury risk by impairing processing, planning and motor execution (Yogev-Seligmann, Hausdorff, \& Giladi, 2008). Moreover, these mental health disorders appropriate attentional resources, limiting the resources 
available for postural control and gait attentional resources, limiting the resources available for postural control and gait (Yogev-Seligmann et al., 2008).

Impaired cognition resulting from depression or anxiety also has an impact on postural stability and gait. During a dual task of working memory and postural control, depressed patients demonstrated impaired postural control during the dual task compared to healthy controls (Doumas et al., 2012). Impaired postural control may put the individual at increased injury risk due to inability to control movement (Paterno et al., 2010), poor start position (Sosnoff, Broglio, Shin, \& Ferrara, 2011), or respond to dynamic perturbations in postural equilibrium (Jones, 2000). Postural stability has been associated with subsequent lower-body injury (Paterno et al., 2010; Witchalls, Blanch, Waddington, \& Adams, 2012) and hypothesized as a mechanism for musculoskeletal injury after concussion (Lynall, Mauntel, Padua, \& Mihalik, 2015). Postural and gait deficits may increase the risk for injury by increasing the likelihood of falls or impairing avoidance or bracing movement.

In addition to postural and gait deficits, reaction and motor speed can also be affected by mental health. Depressed patients demonstrate slowed reaction time (Marazziti et al., 2010) indicating impaired processing speed. Increased reaction time means an individual requires more time to process the stimuli and generate a motor pattern for response. Among athletes and service members who are active in high-velocity activities, increased reaction time can prevent the individual from making the appropriate and timely response, disposing them to injury(Wilkerson, 2012).

Cognitive and motor mechanisms associated with depression and anxiety not only predispose an individual to general injury risk, but also create a vulnerable brain state lowering the threshold for concussion (Kutcher \& Eckner, 2010). For example, global cerebral blood flow is decreased by $12 \%$ among depressed patients when compared to healthy controls (Sackeim et al., 1990). A similar pattern is observed among anxiety patients, with increasing anxiety cerebral blood flow decreased (Gur et al., 1987). As described in Chapter 2.1, decreased blood flow after an impact limits the brain's recovery response due to decreased glucose transport.

\subsubsection{Concussion's Impact on Subsequent Mental Health}

Student-athletes and cadets are at increased risk for injury due to the physical demands of sport and academy training. After any injury there is a psychological response (Putukian, 2016) to cope with the injury. Immediately after a concussion, there is an increase in depression and 
psychological distress compared to healthy controls (Mainwaring, Hutchison, Bisschop, Comper, \& Richards, 2010), that resolves within 14 days. While symptoms resolve immediately postconcussion, there is growing evidence for increased mental health problems later in life. Crosssectional studies of retired athletes (Guskiewicz, Marshall, et al., 2007; Kerr et al., 2012) and military service members (Stein et al., 2015) show increased risk for mood and anxiety disorders among those with a previous concussion. Underlying both acute and chronic outcomes are common neural substrates that are observed in imaging studies of concussion, mood, and anxiety disorders. However, the causal link between concussion and subsequent mental health changes has not been established due to temporality, classification, and generalizability issues.

Because any injury, not only concussion, is associated with increased depression and anxiety (Putukian, 2016), some studies have used musculoskeletal injured patients in addition to non-injured healthy controls. This additional control group helps differentiate the general injury response from a possible concussion specific response. Hutchinson and colleagues studied concussed varsity athletes, healthy physically active college students, and those with musculoskeletal injury. All subjects had a baseline assessment and were followed up for two weeks post-injury. Across the three groups, there were no significant differences observed (Hutchison, Mainwaring, Comper, Richards, \& Bisschop, 2009). The low sample size and significant difference in the proportion of males across groups may have limited the findings. Another study investigating concussed, anterior cruciate ligament (ACL), and healthy controls found that individuals with a concussion or ACL injury had increased depression symptoms post-injury when compared to controls (Mainwaring et al., 2010). ACL patients had a larger increase in depression symptoms from baseline than concussed athletes(Mainwaring et al., 2010). While authors argue that ACL patients had a prolonged increase in depression symptoms, the sampling time points were different for concussed (mean three days post-injury) and ACL patients (mean 11 days post injury), possibly artificially extending the symptom duration. Moreover, the degree and duration of depression symptoms for ACL patients may have been confounded by surgery (Mainwaring et al., 2010) and the longer recovery time (Harris et al., 2014) (6 months) for ACL compared to concussion (7-10 days)(McCrea et al., 2005; Williams et al., 2015).

One other longitudinal study examined depression symptoms post-concussion in high school and collegiate athletes (Kontos, Covassin, Elbin, \& Parker, 2012). Collegiate athletes had 
a significant increase in depression scores at 14 days post-concussion (Kontos et al., 2012). The lack of musculoskeletal and healthy control group limits the interpretation as it is unclear if the increase in depression symptoms are due to a concussion. Similar findings were observed by Vargas and colleagues. In their case-control study of collegiate athletes, $23 \%$ of concussed athletes had increased depression symptoms compared to their baseline (11\%). Non-concussed controls had similar depression rates at baseline (7\%) and follow up (10\%) (Vargas, Rabinowitz, Meyer, \& Arnett, 2015). Current acute studies of concussion do not provide enough evidence for or against a specific concussion psychological response.

At 6 and 10 months after combat-related concussion, service members with concussion had greater depression and PTSD scores than controls (Mac Donald et al., 2017). Importantly, controls were those without concussion and was comprised of combat soldiers who were and were not exposed to blast injury (Mac Donald et al., 2017). This control group likely experienced similar trauma during deployment, and thus the increased number of depression PTSD symptoms among the concussion group cannot be attributed to trauma exposure. Consequently, concussion created a vulnerable brain to PTSD by creating an imbalance between the frontal cortices and limbic areas (Stein \& McAllister, 2009). Unfortunately, this study did not have baseline measures of depression and PTSD. Thus, it is unknown whether the increased depression and PTSD symptoms preceded or resulted from the concussion. One study of military service members did collect baseline depression and PTSD measures before deployment (Stein et al., 2015). Post-deployment $18 \%$ of service members self-reported experiencing a concussion (Stein et al., 2015). Logistic regressions assess for PTSD and depression likelihood at 3 and 9 months post-deployment; controlling for age, sex, race, combat team, number of deployments, PTSD and depression diagnosis and baseline symptoms. Deployment concussion was associated with increased PTSD odds at $3(\mathrm{OR}=1.81 ; 95 \% \mathrm{CI}: 1.32-2.46)$ and 9 months $(\mathrm{OR}=1.48 ; 95 \% \mathrm{CI}$ : 1.21-1.83) post-injury. Deployment-related concussion was not associated with depression or suicide but was associated with increased odds of anxiety at 9 months post-deployment (Stein et al., 2015). Military studies of concussion and mental health have shown a marked increase in PTSD likelihood after concussion (Mac Donald et al., 2017; Stein et al., 2015). However, the study samples were over $90 \%$ male and focused on combat-related concussion, limiting the generalizability of findings to the general population or athletes. 
Studies of concussion within the general population show weaker associations between concussion and mental health. Among individuals presenting to the emergency department with concussion, suicide ideation at three and six months was not related to previous concussion (Bethune, da Costa, van Niftrik, \& Feinstein, 2017). In this study, 22\% completed the three and six month assessments (Bethune et al., 2017). The high rate of drop out may have created sample bias and the lack of pre-injury health information limited the ability to control for confounders. Another study of concussion, found that overall symptom reporting was low and depression symptoms increase up to six months post-concussion and then plateaued (Barker-Collo et al., 2015). Anxiety symptoms are elevated up to six months post-concussion, but then resolve (Barker-Collo et al., 2015). However, the lack of control group makes it unclear whether depression and anxiety symptoms are specific to concussion or injury in general. An ED study of concussion and musculoskeletal injured patients showed no significant differences in depression or PTSD symptoms at one, six, or twelve months post-injury (Losoi et al., 2016). Study design limitations likely influence the associations between concussion and mental health. Prospective investigations accounting for baseline mental health are needed moving forward to better assess for mental health changes after concussion in non-athletes and non-military service personnel.

Within the sub-acute period, there are few studies on psychiatric symptoms and diagnoses. Concussion is a relatively rare event that makes prospective studies difficult. Consequently, many studies do not have a baseline or pre-injury data on their participants. This information not only establishes whether a concussion preceded mental health changes but also allows for the controlling of potential confounders. Future prospective studies of concussion and the evolution of mental health changes are needed to assess whether concussion is a causal mechanism.

Studies of retired athletes show a pattern of increased depressive symptoms and diagnosis. Among studies of retired professional American football (NFL) players, a greater number of concussions is associated with increased likelihood of a present or previous depression diagnosis (Guskiewicz, Marshall, et al., 2007; Kerr et al., 2012). A dose-response relationship was observed, with increasing likelihood of depression diagnosis with increasing number of concussions (Figure 2.6.1). Another study of NFL players found that a concussion history correlated with the cognitive symptom domain of the Beck Depression Inventory (BDI), but not the affective or somatic domains (Didehbani, Munro Cullum, Mansinghani, Conover, \& 
Hart, 2013). This symptom evaluation suggests that depression post-concussion may be driven more by cognitive features of depression. Further, results from the imaging studies described above, demonstrate a breakdown in the structure and function of the frontal lobe, responsible for cognition. Outside of professional athletes, few chronic studies have been completed. An online survey of college students found that the number of self-reported concussion correlated BDI total symptoms scores (rho $=0.48 ; \mathrm{p}<0.001$ ) (Vynorius et al., 2016).

Cross-sectional studies of professional athletes and college students suggest an association between concussion and depression. The dose-response between the number of previous concussions and likelihood of depression diagnosis adds to the strength of evidence for concussion to be a possible causal mechanism. However, due to the cross-sectional design, it is unclear whether the concussion proceeded depression symptoms.

Figure 2.6.1. Depression Diagnosis by Concussion History

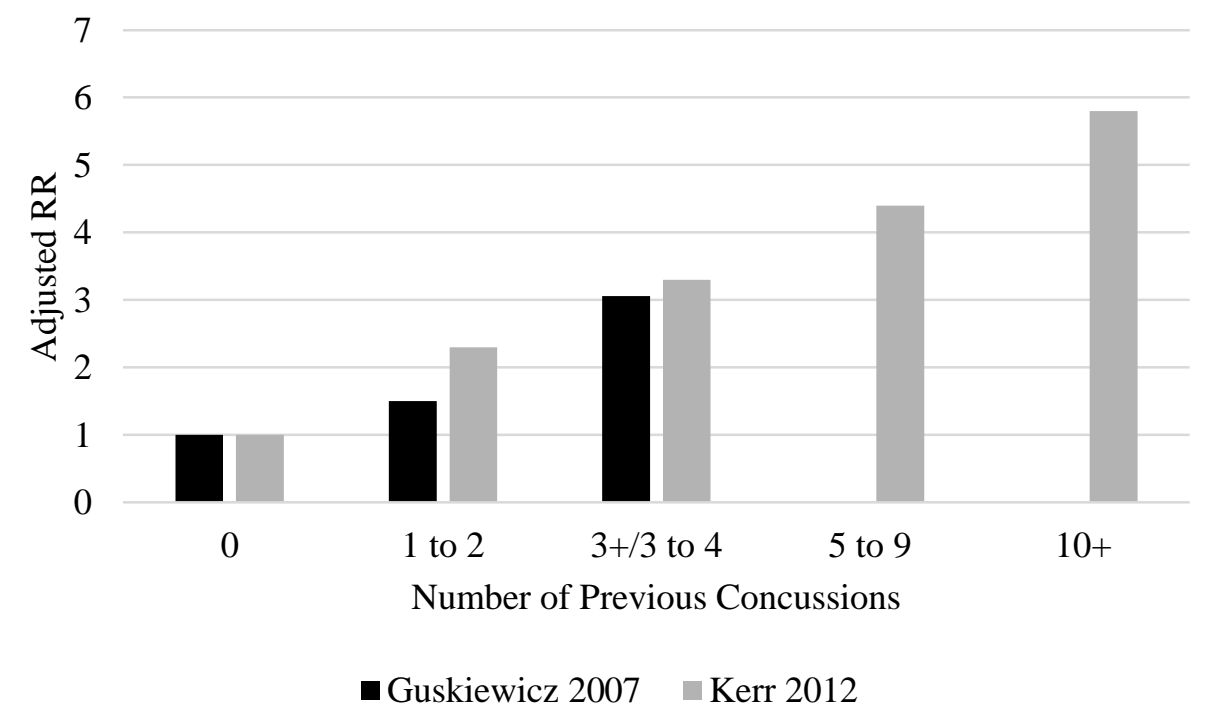

Adapted from Guskiewicz et al 2007(Guskiewicz, Marshall, et al., 2007) and Kerr et al 2012(Kerr et al., 2012)

\subsubsection{Summary}

Mental health, especially depression and anxiety, have been associated with increased injury risk. Cognitive, motor, and brain vulnerability associated with mental health disorders may predispose an individual to concussion. While postulated to be associated with concussion risk, no prospective study has investigated concussion likelihood after mental health diagnosis or increased symptomatology. 
Acute and chronic studies of concussion have demonstrated an increased association between concussion and depression, anxiety, or PTSD. However, few studies have been prospective, limiting the evidence for concussion causing subsequent mental health changes. Additionally, the majority of study have been conducted in male or professional athletes, limiting generalizability. Future studies should have a longitudinal design, focus on mental health evolution within the sub-acute window post-injury, and include females and non-professional athletes.

\subsection{United States Service Academies and Cadets}

There are five United States (U.S.) service academies, the U.S. military academy (USMA), U.S. air force academy (USAFA), U.S. coast guard academy (USCGA), U.S. naval academy (USNA), and U.S. merchant marine academy. All five of these academies are federal institutions designed to educate and train officers of the U.S. armed forces. USMA, USAFA, and USNA report to the Department of Defense, while USCGA reports to Homeland Security, and the merchant marines report to the Department of Transportation. However, under wartime USCGA can be placed under control of the Navy and thus indirectly the Department of Defense. Therefore, for this dissetation only USMA, USAFA, USNA, and USCGA will be considered and referred to as service academies. Additionally, the term "cadets" will refer to Service Academy members at the U.S. Service Academies.

Once accepted, cadets will begin at the academy with basic training. This six-week long period prior to the first academic year that introduces cadets to academy and military life. Cadets will be sworn in, learn first aid, honor and ethics, and military values (Roeder, 2017). During this period, cadets experience increased stress due to the new regiment, lifestyle, along with physical and mental demands. Perceived stress during basic training increased $32 \%$, relative to baseline(Lee, Meehan, Robinson, Mabry, \& Smith, 1992). From 5:00 AM until 10:00 PM a cadet's day during basic training is scheduled (Miller, Shattuck, \& Matsangas, 2010) (Figure 2.7.1). Each day consists of physical training, classes, and athletics. Once out of basic training, the rigorous schedule is maintained, but with slightly different hours (West Point, 2014) (Figure 2.7.1). The below quote describes a few of the strict regiments cadets must adopt. 
Figure 2.7.1. Cadet's Daily Schedule

\begin{tabular}{|c|c|}
\hline Schedule During USMA Basic Training & USMA Academic Day \\
\hline 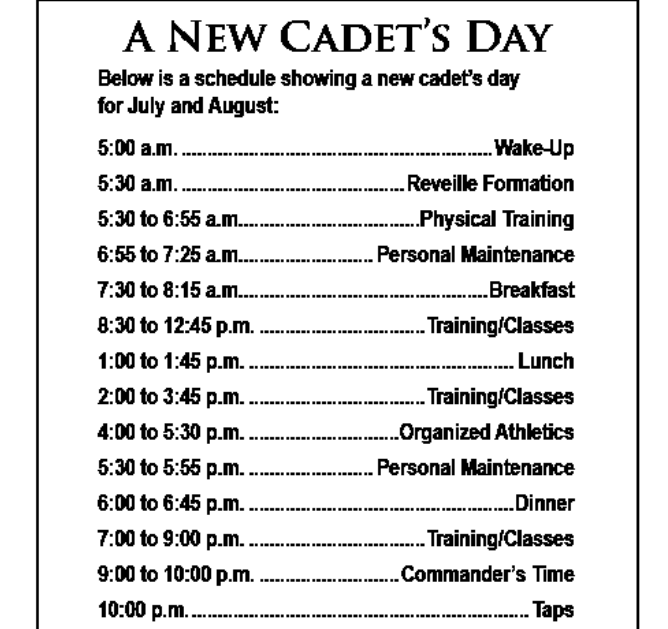 & \begin{tabular}{|ll}
$06: 30$ & Reveille \\
$06: 55-7: 30$ & Breakfast Formation (mandatory) \\
07:35-11:45 & Class (55 minutes per class) or study time \\
12:05-12:40 & Lunch \\
12:45-13:40 & Commandant Dean Time \\
13:50-15:50 & Class or study \\
16:10:17:45 & Intramural, club or intercollegiate athletics; parades; extracurricular activities; or free time \\
18:30-19:15 & Supper (optional except Thursdays) \\
19:15-19:30 & Cadet Duties \\
19:30-20:30 & Study conditions Extracurricular activities \\
20:30-23:30 & Study time \\
$23: 30$ & Taps \\
$24: 00$ & Lights Out \\
\end{tabular} \\
\hline
\end{tabular}

The particular entrance requirements at the academies, create individual characteristic differences across cadets and students at civilian secondary schools. At the academies, females are a minority, composing $20-36 \%$ of the cadet population while at civilian institutions females represent over $50 \%$ of the student population (Kirby, Thie, Naftel, \& Adelson, 2010). Among NCAA athletes at civilian institutions, males compose $56.6 \%$ of the student athlete population (Johnson, 2014). In addition to demographic differences, cadets differ from students and studentathletes at civilian institutions in academic achievement, physical fitness, and medical histories.

The U.S. service academies are continually ranked as some of the best higher-education institutions in the U.S. The low acceptance rate and high academic standards fosters recruitment of high achieving students. Based on current CARE data (CARE Consortium, unpublished data), service academy cadets had significantly (all p's < 0.001) higher high school GPAs and ACT scores compared to the non-academy sites (Figure 2.7.2). Additionally, overall academy graduation rates range from $79-89 \%$, compared to an overall $66 \%$ rate at civilian institutions (U.S. Department of Education, 2017). Comparatively, the service academy graduation rate is similar to the NCAA Division I graduation rate of scholarship student-athletes at $82 \%$ (National Collegiate Athletic Association, 2017). The greatest proportion of cadets (10-15\%) drop out 
during their first year at the academy (Kirby et al., 2010). Overall, graduation rates of cadets and civilian student athletes are similar. However, there are distinct differences in academic achievement and performance between cadets and student athletes.

Figure 2.7.2. Academic Performance across Cadets and Non-Cadets

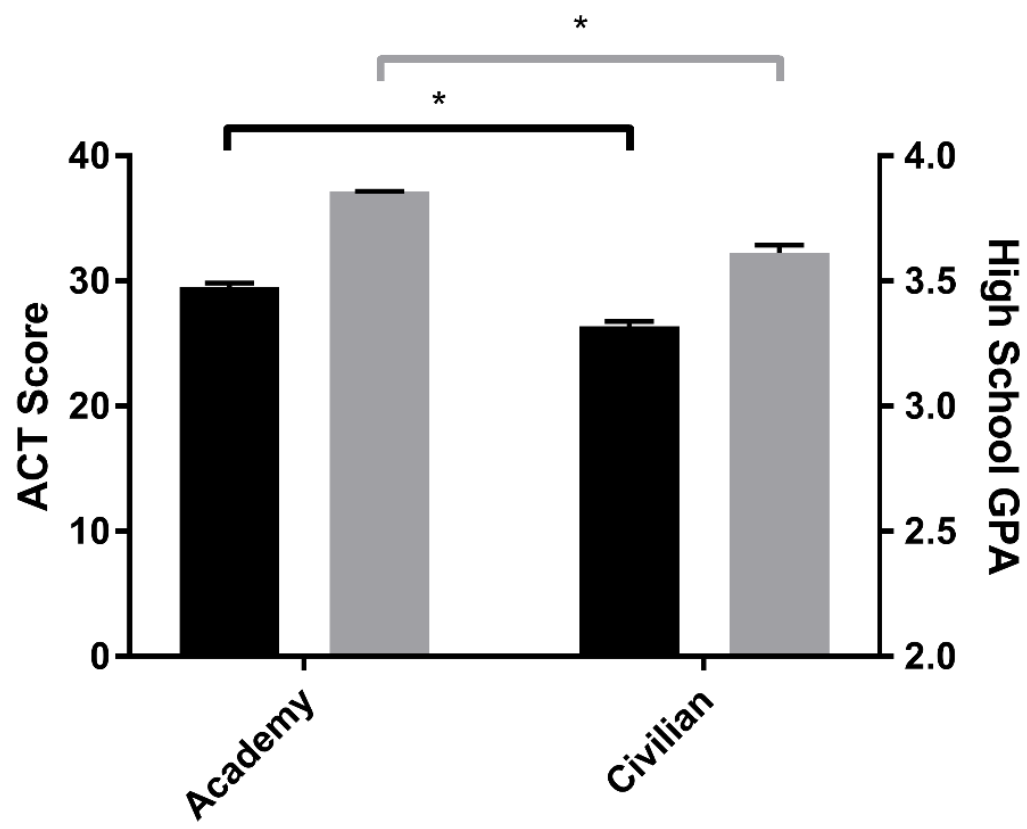

- ACT Score

\section{High School GPA}

In addition to academic standards, the service academies also have physical fitness standards that apply to every cadet on campus. Academies require the candidate fitness assessment (CFA) or the physical fitness exam (PFE) (United States Military Academy, 2017). Once enrolled, cadets continue to take physical fitness tests in order to ensure they maintain the minimum fitness level. The aerobic fitness test (AFT) and physical fitness test (PFT) are required examinations each semester(O'Connor, Campbell, McGinty, \& Zupan, In Prep). The only exceptions are NCAA athletes and/or those cadets who score within the top $10^{\text {th }}$ percentile on either test.

Beyond physical fitness assessments, each academy requires their cadets to participate in an athletic activity. The cadet will compete at either at the varsity (NCAA), competitive club, or intramural level. Military, or academy, training exercises are also part of the physical regiment. Combative and boxing courses are required of all first year cadets. Additionally, in 2016, boxing 
became a new requirement for female cadets (Philipps, 2015). With all combat roles now open to both sexes, boxing at the academy was the only activity that females did not complete while their male peers did. Additional military specific training exercises take place during the summer or academic years (United States Military Academy, 2016) on top of physical assessment and athletic requirements. Consequently, service academy cadets have greater physical activity demands and a greater variety of activity than civilian NCAA athletes.

A major application requirement for admittance to a service academy is a medical examination. The medical requirement ensures the cadet's health, limits time loss from duty, successful completion of required training, and healthy adaptation to various military environments. The medical requirements are meant to certify an individual is capable of performing their military duties (Department of Defense Instruction, 2010)

Many medical conditions associated with concussion or recovery exclude a candidate from being accepted to a military academy. For example, moderate traumatic brain injury is exclusionary unless the individual shows no residual dysfunction after 12 months post-injury. Also, mild traumatic brain injury is exclusionary unless there is no residual dysfunction one month post-injury and has no history of post-concussive syndrome (Department of Defense Instruction, 2010). Recent research is suggesting concussion is associated with increased risk for another injury (Department of Defense Instruction, 2010) and increasing concussion history is associated with prolonged recovery after an incident concussion (Department of Defense Instruction, 2010). There are currently no regulations surrounding these issues, but $18 \%$ of incoming cadets have a previous diagnosed or undiagnosed concussion (O'Connor et al., 2016). While the medical requirements do not prevent all candidates with a TBI history from entering the service academy, the requirements likely limit the number of individuals who have had a complicated head injury.

Other comorbidities including headache disorders, migraine, sleep disorders, attention deficit disorder (ADD)/Attention Deficit Hyperactivity Disorder (ADHD) learning disabilities, mood disorders anxiety disorders, and substance dependence/abuse are also exclusionary medical criteria (Department of Defense Instruction, 2010). ADHD has been associated with increased lifetime concussion prevalence among high school athletes (Iverson et al., 2016) but the cross-sectional study design limits causal inferences and it remains unclear whether learning disabilities increase concussion risk or concussion causes learning disabilities. Depression, 
anxiety, sleep (Kutcher \& Eckner, 2010), and headache/migraine (Lau et al., 2009) disorders have all been associated with concussion and recovery. However, similar to learning disabilities it is unclear their causal role.

While the medical admission requirements at the Service Academies make the cadet population substantially different from traditional NCAA athlete, the minimization or complete reduction of medical and psychological comorbidities associated with concussion risk and recovery provides researchers with a unique opportunity. When an individual does not have any known risk factors for concussion, only environmental characteristics remain to influence concussion risk. Consequently, researchers can assess how certain activities, training, and athletics influence concussive injury risk. Furthermore, without these potential confounders, researchers can examine the singular relationship between concussion and outcomes. By investigating cadets, researchers can better understand how an incident concussion impacts symptoms, cognitive, and emotional functioning within acute and sub-acute timeframes.

\subsubsection{Summary}

U.S. service academies are unique from civilian higher education institutions, whereby the institutional environment and individual characteristics differ. No previous studies have comprehensively studied the natural history of concussion and recovery among cadets. Examining the activities of cadets will elucidate how athletic competition level, military training, and outside activities cause concussion. Additionally, cadets are extremely healthy individuals due to the physical and medical standards. Consequently, researchers are better able to understand the true consequences of concussion in the absence of other confounders. 


\section{CHAPTER 3: Dissertation Projects}

\section{Chapter 3.1 Concussion Assessment, Research and Education Consortium}

In 2014, the National Collegiate Athletic Association and the U.S. Department of Defense (DoD) established a partnership known as the Grand Alliance. The partnership funded the Concussion, Assessment, Research, and Education (CARE) Consortium, a multi-site investigation on the natural history of concussion. Between 2014 and 2017, 30 Division I, II, and III schools, from a variety of conferences, and United States service academies joined the CARE consortium. Currently, three service academies: United States Air Force Academy (USAFA), United States Coast Guard Academy (USCGA), and the United States Military Academy (USMA) are active sites.

\subsubsection{Participants}

Within the CARE Consortium, three United States services academies submitted data for analysis. Two United States services academies are Division I institution and the third is a Division III institution. In contrast to the civilian institutions that enrolled only varsity athletes, all cadets on campus were eligible to participate in the CARE study.

All cadets from the participating service academies are required to participate in an athletic activity at the intercollegiate varsity, competitive club, or intramural athletics (i.e. intracollegiate activities) level. Competitive club athletes are non-NCAA athletes who play on a competitive team and compete in non-NCAA sanctioned intercollegiate competition. 
Finally, intramural athletes are engaged in activities where competition occurs between companies within the academy. Classification of activities within competition level has been outlined in the Table 3.1.1. To evaluate and control for sub-concussive impact, varsity level cadets are contact, limited-contact, and non-contact sports using exposure groups defined by Rice in 2008(Rice et al., 2008). Due to the multitude and lack of data on exposure, competitive club and intramural sports were not categorized into contact exposure groups.

Table 3.1.1. Service Academy Athletic Activities by Competition Level

\begin{tabular}{|l|l|l|}
\hline Varsity & Competitive Club & Intramurals \\
\hline Baseball (Men) & Boxing** & Area Hockey \\
Basketball & Cheerleading*** & Basketball \\
Crew/Rowing (Women) & Climbing & Boxing** \\
Cross Country & Cycling & Dodgeball \\
Diving & Equestrian & Field Ball \\
Fencing** & Fencing** & Flag Football \\
Field Event & Judo & Flickerball \\
Football (Men) & Marathon & Functional Fitness/CrossFit \\
Golf (Men) & Orienteering & Goaltimate \\
Gymnastics (Men or Coed) & Pistol & In-Line Hockey \\
Hockey (Men) & Rowing/Crew(Men) & Mountaineering \\
Lacrosse (Men or Coed) & Rugby & Paintball \\
Rifle & Sailing & Pass-N-Go \\
Soccer & Sprint Football (Men) & Rodeo \\
Softball (Women) & Strength/Powerlifting & Skiing \\
Swimming & Team Handball & Soccer \\
Tennis & Triathlon & Softball \\
Track & Volleyball (Men) & Squash \\
Volleyball (Women) & Submission Grappling \\
Water Polo (Men) & Ultimate Frisbee \\
Wrestling (Men) & \\
$*$ Coed unless otherwise noted & Volleyball** Polo \\
$* *$ Sports may be listed under more than one competition level depending on the academy \\
\hline \multirow{2}{*}{ Cheerleading collected as a varsity sport in CARE study } \\
\hline
\end{tabular}

\subsubsection{Assessment Timeline}

Prior to data collection at each academy, all research staff were trained on a standardized protocol for preseason baseline testing and post-injury assessments. Protocols approved by the site-level Institutional Review Board (IRB) of each performance site also underwent review and approval by the DoD Human Research Protections Office (HRPO)(Broglio, McCrea, et al., 
2017). All cadets were presented with the study and if willing, were consented. Enrolled cadets received an annual baseline assessment.

\section{Baseline}

Baseline assessments included self-reported demographic information and medical history (Broglio, McCrea, et al., 2017). In addition to common demographic information (e.g. age, height, weight, sex) data were collected on household income, years in the primary sport, age, and race. The medical history included information about personal and familial medical conditions, concussion history, and psychological history. Concussions were defined based on evidence-based guidelines(Broglio, McCrea, et al., 2017) indicating the injury as a change in brain function following a force to the head, which may or may not be accompanied by temporary loss of consciousness. Using this definition cadets were asked to list all previously diagnosed and undiagnosed concussions. Next, each cadet underwent a neurocognitive evaluation, neurological screening, postural stability, psychological distress assessment, and selfreported symptom evaluations.

\section{Post-Injury}

Concussion Definition: Concussion will be defined using a consensus definition produced by a U.S. Department of Defense funded evidence-based guidelines group(Carney et al., 2014) and endorsed by the NCAA Concussion Management Guidelines 2014, which defines concussion as a change in brain function following a force to the head, which may be accompanied by temporary loss of consciousness, but is identified in awake individuals with measures of neurologic and cognitive dysfunction. Identification and assessment of concussive events are completed by the team's athletic trainer or academy's clinician. A physician confirmed each diagnosis. All diagnoses were made by local medical staff using a clinical exam, supported by objective assessments (Broglio, McCrea, et al., 2017).

Once an athlete was diagnosed with a concussion, a series of post-injury assessments occur (Figure 3.1.1). Assessments occur within 6 hours and 24-48 hours post-injury. After 48 hours the cadet completes a symptom checklist daily until they are asymptomatic. Asymptomatic is not defined as the complete absence of symptoms, but the time at which the cadet is cleared by the clinical team to begin the return-to-activity (RTA) protocol. At this point, the cadet completes an asymptomatic assessment and begins the RTA protocol. At the conclusion of the protocol, the cadet will complete the RTA assessment. Finally, at 6 months post-injury the cadet 
completes another assessment. If the cadet does not graduate or leave the academy, they will complete another annual baseline. Table 3.1.2 outlines the assessment tools used at each time point. Details of each assessment are described below. 
Figure 3.1.1 CARE Assessment Timelines

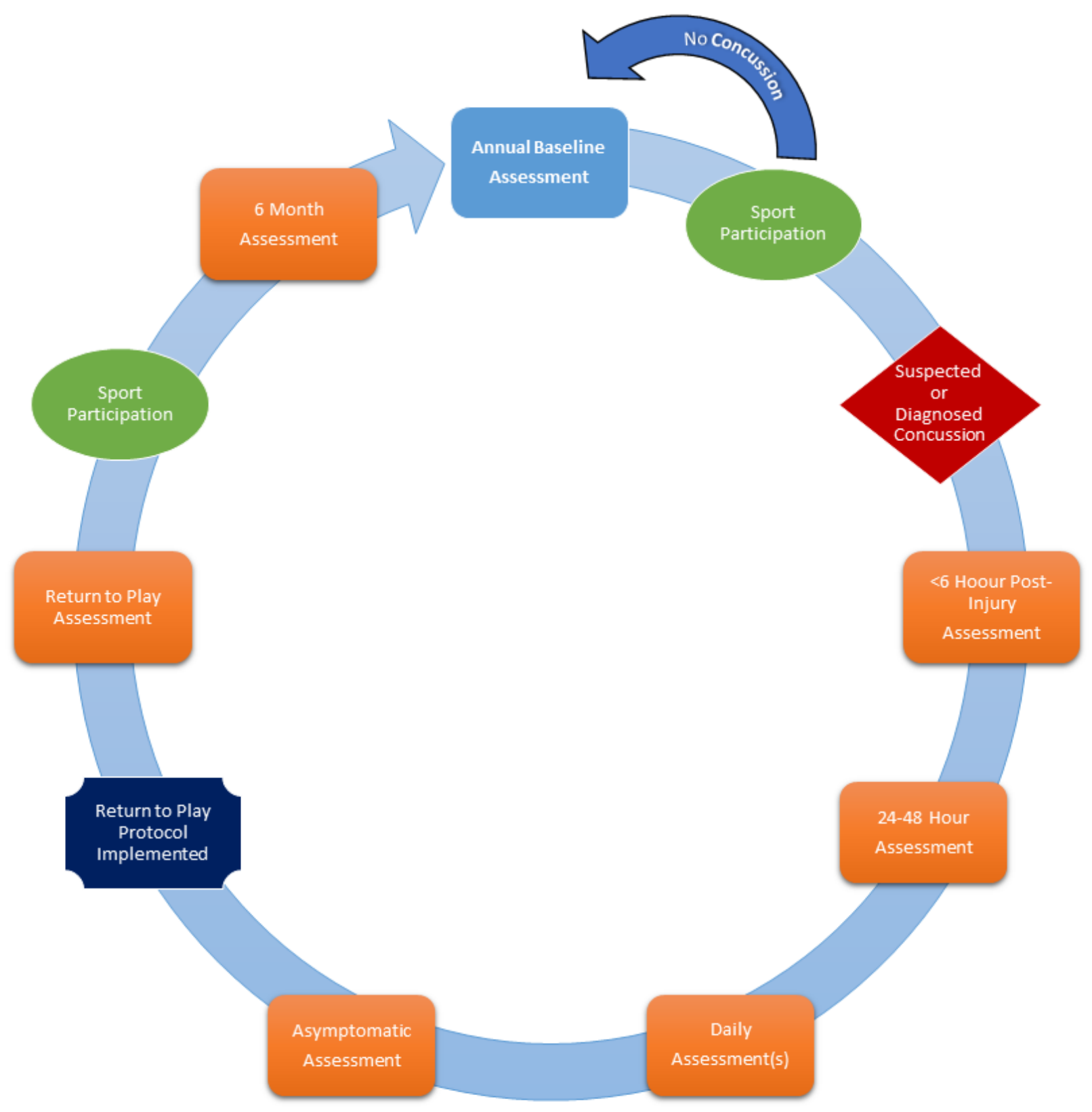


Table 3.1.2. Assessments by Time Point

\begin{tabular}{|c|c|c|c|c|c|c|c|c|c|c|}
\hline & \multirow{2}{*}{$\begin{array}{c}\text { Neurocognitive } \\
\text { ImPACT } \\
\end{array}$} & \multirow{2}{*}{$\begin{array}{c}\text { Neurological } \\
\text { Screening } \\
\text { SAC }\end{array}$} & \multirow{2}{*}{$\begin{array}{c}\text { Postural } \\
\text { Stability } \\
\text { BESS }\end{array}$} & \multicolumn{2}{|c|}{ Symptoms } & \multirow{2}{*}{$\begin{array}{c}\text { Risk } \\
\text { Taking } \\
\\
B S S S\end{array}$} & \multirow{2}{*}{$\begin{array}{c}\text { Intelligence } \\
\text { WTAR } \\
\end{array}$} & \multicolumn{3}{|c|}{ Interview } \\
\hline & & & & $S C A T$ & BSI-18 & & & $\begin{array}{c}\text { and Medical } \\
\text { History }\end{array}$ & $\begin{array}{c}\text { Injury } \\
\text { Characteristics }\end{array}$ & $\begin{array}{c}\text { Return to } \\
\text { Activity }\end{array}$ \\
\hline Baseline & $\mathrm{x}$ & $\mathrm{x}$ & $\mathrm{x}$ & $\mathrm{x}$ & $\mathrm{x}$ & $\mathrm{x}$ & $\mathrm{x}$ & $\mathrm{x}$ & & \\
\hline$<6$ Hours & & $\mathrm{x}$ & $\mathrm{x}$ & $\mathrm{x}$ & & & & & $\mathrm{x}$ & \\
\hline 24-28 Hours & $\mathrm{x}$ & $\mathrm{x}$ & $\mathrm{x}$ & $\mathrm{x}$ & $\mathrm{x}$ & & & & & \\
\hline Daily Symptoms & & & & $\mathrm{x}$ & & & & & & \\
\hline Asymptomatic & $\mathrm{x}$ & $\mathrm{x}$ & $\mathrm{x}$ & $\mathrm{x}$ & $\mathrm{x}$ & & & & & \\
\hline $\begin{array}{l}\text { Return to } \\
\text { Activity }\end{array}$ & $\mathrm{x}$ & $\mathrm{x}$ & $\mathrm{x}$ & $\mathrm{x}$ & $\mathrm{x}$ & & & & & $\mathrm{x}$ \\
\hline 6 Month & $\mathrm{x}$ & $\mathrm{x}$ & $\mathrm{x}$ & $\mathrm{x}$ & $\mathrm{x}$ & & & & & \\
\hline
\end{tabular}




\subsubsection{Measurement Tools}

The Sport Concussion Assessment Tool (SCAT) (McCrory, Meeuwisse, Aubry, Cantu, Dvořák, Echemendia, Engebretsen, Johnston, Kutcher, Raftery, \& Sills, 2013; McCrory et al., 2017) symptom scale, Brief Symptom Inventory (BSI-18) (Meachen et al., 2008), Standardized Assessment of Concussion (SAC) (McCrea et al., 1998), Balance Error Scoring System (BESS) (Guskiewicz, Ross, Ross, \& Guskiewicz, 2001), and Immediate Post-Concussion Assessment and Cognitive Testing (ImPACT) (Iverson et al., 2010) were the primary assessments administered at various time points. ImPACT and SAC were the primary neurocognitive evaluations, BESS evaluated postural stability, the SCAT assesses post-concussive symptoms, and the BSI-18 evaluated psychological health symptoms. Each site received training from the University of Michigan on how to administer each test. Furthermore, additional refreshers were completed at annual consortium-wide meetings and monthly teleconferences.

Sport Concussion Assessment Tool (SCAT): The SCAT symptom scale is a standardized assessment for evaluating concussion. The symptom scale contains 22 symptoms and a 7 point Likert scale ranging from "none" to "severe" (McCrory, Meeuwisse, Aubry, Cantu, Dvořák, Echemendia, Engebretsen, Johnston, Kutcher, Raftery, \& Sills, 2013; McCrory et al., 2017). The SCAT symptom scale has been shown to be fairly reliable with and ICCs of 0.62 and 0.43 at 7 and 196 day intervals (Schatz, 2010).

Brief Symptom Inventory (BSI-18): The BSI-18 is an 18 item scale designed to measure psychological distress across four domains: depression, anxiety, somatization, and total distress (Meachen et al., 2008). Among a traumatic brain injury sample retested at a median interval of one year, the BSI-18 was shown to have good reliability, with Pearson product moment correlations as follows: total score, 0.66; somatization, 0.67; depression, 0.63; and anxiety, 0.57 (Chin et al., 2016).

Standardized Assessment of Concussion (SAC): The SAC is an acute mental status exam consisting of four domains: orientation, immediate memory, concentration, and delayed recall (McCrea et al., 1998). The SAC has been shown to have high sensitivity and specificity (McCrea, 2001). 
Balance Error Scoring System (BESS): The BESS measures postural stability on a firm and foam surface across three stances (i.e. double leg, single leg, and tandem). Scores reflect the number of errors (e.g. losing balance) committed during the 20 second test period (Guskiewicz et al., 2001). The BESS has been shown to have good test-retest reliability (0.87-0.97 ICC) (Iverson, Gaetz, et al., 2009).

Immediate Post-Concussion Assessment and Cognitive Testing (ImPACT): While multiple neurocognitive tests were allowable under the CARE protocol (Broglio, McCrea, et al., 2017), each academy implemented the ImPACT, a computerized neurocognitive assessment that tests verbal memory, visual memory, motor speed, and reaction time performance (Iverson et al., 2010). When collegiate athletes were tested across a two-year interval, ICCs ranged from fair to good for verbal memory (0.46), visual memory (0.65), processing speed (0.74), and reaction time (0.68) (Schatz, 2010).

Brief Sensation Seeking Scale (BSSS): The BSSS is an abbreviated measure of risk taking and sensation seeking. The eight questions include two items representing each of the four aspects of sensation seeking (Stephenson, Hoyle, Palmgreen, \& Slater, 2003). The four aspects of sensation seeking include: experience seeking, disinhibition, thrill and adventure seeking, and boredom susceptibility (Stephenson et al., 2003). The BSSS has high internal consistency $(r=0.76)$ and has a strong associated with risk-taking behaviors like drug attitudes and behaviors (Hoyle, Stephenson, Palmgreen, Lorch, \& Donohew, 2002). Sex differences have been reported previously, with males reporting greater BSSS scores than females (Hoyle et al., 2002; Stephenson et al., 2003). 


\subsection{Project 1: Concussion Risk Factors}

\subsubsection{Introduction}

Concussion, or mild traumatic brain injury (mTBI), is a prevalent injury among athletes and military service members. An estimated 1.6-3.8 million sport and recreation-related concussion occur annually (Langlois et al., 2006). Concussion has also been called the signature injury of recent wars, affecting 14.9\%-22.8\% service members (Hoge et al., 2008; Terrio et al., 2009; Wilk et al., 2010). The high concussion prevalence among athletes and service members is a growing public health concern.

Concussion epidemiological studies have been conducted among athletes (Zuckerman et al., 2015), service members (Cameron et al., 2012; Helmick et al., 2015), or within emergency departments (Bazarian et al., 2005) to better understand the concussion burden within each subpopulation. However, to date, concussion risk factors have only been characterized separately within each subpopulation and typically only related to a particular mechanism (e.g. sportrelated). Athletes and service members are not immune to concussions outside of the sport arena or combat. For example, $80 \%$ of concussions sustained by service members occurred outside of active war-zones (Cameron et al., 2012); likely due to a combination of sport, recreation, and military training (e.g. boxing) related activities. Understanding and identifying individual characteristics that increase overall concussion risk is essential to fully characterizing concussion and its burden.

To reduce concussive injuries, recent research has focused on identifying individual-level concussion risk factors. Among high school and collegiate athletes, females have 1.5 to 2.1 times greater concussion risk than their male peers (Covassin, Swanik, \& Sachs, 2003; Lincoln et al., 2011). Females are hypothesized to have greater risk due to a combination of physical, psychological, and physiological differences (Dick, 2009). Female concussion risk in the military is relatively unknown due to small sample sizes. However, due to evolving female combat roles, new studies investigating female concussion risk among service members are essential.

In addition to sex, medical comorbidities such as learning disorders, migraine headaches (Kutcher \& Eckner, 2010), previous concussion (Abrahams et al., 2014), and baseline symptoms (Schneider, Meeuwisse, Kang, Schneider, \& Emery, 2013) influence concussion risk. Relative to other medical comorbidities, having a previous concussion had the strongest evidence for increasing subsequent concussion risk (Abrahams et al., 2014). A systematic review of 
concussion risk factors found that 10 of 13 studies found increased subsequent concussion risk in athletes with a previous concussion (Abrahams et al., 2014). The association between previous concussion and incident concussion was observed in a variety of sports (Abrahams et al., 2014). However, nine of the ten studies enrolled all male or $99 \%$ male participants. While previous concussion appears to increase incident concussion risk, further research is needed to confirm this relationship within both sexes.

Baseline symptoms increased concussion risk in youth ice hockey players (Schneider et al., 2013). Increased symptoms also mediate somatization symptoms, whereby higher baseline somatization symptoms increase concussion-related symptom burden or reporting (Nelson et al., 2016). While pre-injury somatization correlates with longer recovery duration (Nelson et al., 2016), somatization may also increase concussion risk by increasing concussion-related symptom burden or reporting behavior. Thus increased baseline concussion and somatization symptoms may increase concussion risk.

Other psychological symptoms and disorders such as anxiety and depression, have also been hypothesized to increase concussion risk (Kutcher \& Eckner, 2010). A recent study of varsity collegiate athletes found that increased baseline anxiety symptoms increased the likelihood of any injury type in the following athletic-season for both sexes (Li et al., 2017). Increased depression symptoms only increased subsequent injury risk for males, not females (Li et al., 2017). Since injuries included concussive and non-concussive injuries (e.g. ankle sprain), it is unclear whether increased psychological symptoms have a specific influence on concussion likelihood. Further examination is needed to determine the role of psychological symptoms on subsequent concussion risk.

The current study aims to characterize concussion risk and rate among service academy members (cadets) at the United States Service Academies. Risk factors for any concussion along with sport-related, training-related, and free time-related concussions will be characterized. Examining an individual's risk for a concussion across multiple domains, rather than a singular mechanism (e.g. sport-related concussion), will yield a more comprehensive understanding of concussion incidence. Consequently, better targeted prevention and screening initiatives may be developed, especially for females.

We hypothesize that female cadet athletes will have increased sport-related concussion risk (Covassin et al., 2003; Lincoln et al., 2011). Relative to females, males are at increased risk 
for military-related concussions (Hoge et al., 2008) and concussions presenting to emergency departments (Bazarian et al., 2005; Cassidy et al., 2004). Thus, we hypothesize that males will have greater training-related and free time-related concussion risk and rates. Previous concussion and increased baseline psychological symptoms were also expected to increase concussion risk. Finally, given the strict oversight of boxing training, we hypothesize that boxing will have lower risk than varsity athletics. Furthermore, while the introduction of female boxing would increase female boxing-related injuries, the overall concussion risk for females would remain unchanged.

\subsubsection{Methods}

\subsubsection{Study Design}

The current study is part of a larger joint effort by the U.S. Department of Defense (DoD) and National Collegiate Athletic Association (NCAA). This partnership funded the Concussion, Assessment, Research, and Education (CARE) Consortium, a multi-site investigation on the natural history of concussion. A detailed description of the CARE Consortium has been published previously (Broglio, McCrea, et al., 2017). Site-level Institutional Review Board (IRB) and DoD Human Research Protections Office (HRPO) review were obtained (Broglio, McCrea, et al., 2017) and all participants provided consent before testing.

Between 2014 and 2017 all cadets were eligible to enroll in CARE. Cadets, commonly referred to as tactical athletes, carry many similarities as varsity athletes, allowing for comparisons. Before each academic year, each enrolled cadet completed a baseline assessment. Baseline assessments included self-reported demographic information, medical history, sport participation history, and social economic status questions (Broglio, McCrea, et al., 2017).

Incident concussions were defined by evidence-based guidelines (Carney et al., 2014). After a diagnosed concussion, research staff recorded the injury's characteristics, including the date and time of injury and the activity the cadet was participating in at the time of injury. These activities are categorized into sport concussions (varsity, club, intermural level), training (physical education, military training, or boxing), or free time-related concussions. Free timerelated concussions were defined as those not occurring during any structured Service Academy activity. Since all cadets must participate in sport and training activities (O'Connor et al., 2018) all cadets were at risk for sport and training-related concussions.

\subsubsection{Statistical Analysis}

Incident Concussion Definition and Baseline Selection 
An incident concussion was defined as any newly diagnosed concussion occurring during the study period. For cadets who sustained multiple incident concussions during the study period, each concussion was coded. For example, a cadet sustaining a concussion during football and a second concussion during a training exercise would have one positive indicator for sport-related concussion and one for training-related concussion. Concussion risk was estimated across all concussions and for each concussion type (sport-related, training-related, free time-related).

Because cadets completed annual assessments, there were multiple baselines available for cadets who participated in the CARE study for more than one year. For those cadets who did not sustain an incident concussion, their most recent baseline was utilized. For those cadets who sustained a concussion, the baseline that preceded their first concussion was used.

For concussion rate estimations, person-time was defined as the years between the first baseline completed and the date of injury. If cadets were not injured, the date of data extraction (June 1, 2017) or date of graduation was used as the end of exposure date. Rates were only calculated for the first incident concussion sustained by the cadet.

\section{Missing Data}

Out of 800 injuries, there were 81 injuries missing an injury activity designation. There was no association between sex or site with the likelihood of missing injury activity data. School year was significantly associated with missing injury activity data $\left(X^{2}(2)=178.07 ; \mathrm{p}<0.001\right)$. The 2014-2015 academic year had the greatest proportion of missing data that decreased with each subsequent study year. The larger proportion of missing data in the 2014-2015 academic year is likely due to the rapid start of the study. Also, sport level (varsity, club, intramural) was associated with missing injury activity data $\left(X^{2}(3)=41.98 ; \mathrm{p}<0.001\right)$. Injuries among cadets who skipped the sport level question were also more likely to have missing data for injury activity. Analysis

Univariate tests first assessed for associations between baseline indicators, concussion and, sex. Chi-squared tests assess for associations between nominal variables while independent sample t-tests asses for differences in continuous variables. The Satterthwaite t-test was used if the variances across groups were unequal. Furthermore, for non-normally distributed variables the non-parametric Wilcoxon test was used. Due to the large sample size in the study, effect sizes were estimated for each significant finding. Phi $(\Phi)$ was the effect size estimated for categorical 
variables with more than two levels with small, medium, and large effect sizes were defined as 0.1, 0.3, and 0.5, respectively (Cohen, 1988). For continuous variables and nominal variables with only two levels, Cohen's d was calculated. Odds ratios were converted to Cohen's d effect sizes using the following formula from Chinn and colleagues(Chinn, 2000):

$$
d=\frac{\ln (O R)}{1.81}
$$

Effect size magnitudes for Cohen's d were defined as 0.3, 0.5, and 0.8 for small, medium, and large magnitudes (Cohen, 1988). Finally, the effect size, r, was estimated when non-parametric tests were used.

Generalized mixed models were used to estimate concussion risk across multiple variables. Because concussion was a rare event, occurring in less than $10 \%$ of cadets, odds ratios were assumed to approximate relative risk (Rothman, 2012). Accounting for clustering effect of Service Academy a random effect of academy was included in each model. Each concussion model first assessed the random effect of academy in an empty model without any covariates. For each concussion type, the random effect of academy was significant and thus was included in each model.

The maximum likelihood method, Laplace, was used to identify a final model. Each predictor was added to the model individually. The only exception was the Brief Symptom Score-18 (BSI-18) sub-scores which were added simultaneously. For each variable to remain in the model, it must be significant, the likelihood ratio test significant, and the BIC value lower than the reference model. After a final model was selected, a generalized mixed model was fit using the pseudo-likelihood method to confirm the significant predictors. Model selection tables are included in the supplementary material. All analyses were completed using SAS version 9.4 (Cary, NC).

\subsubsection{Results}

\subsubsection{Cadet Characteristics}

Between 2014 and June 2017, three Service Academies contributed data from 10,604 (n $=2421$ female $)$ cadets with 4,581 $(\mathrm{n}=934)$ at site $1,5,066(\mathrm{n}=1239$ female $)$ at site 2 , and $953(\mathrm{n}$ $=348)$ at site 3 .

\subsubsection{Concussion Characteristics}

A total of $800(n=301$ female $)$ concussions occurred among 738 cadets. There were 679 $(\mathrm{n}=253$ female), $56(\mathrm{n}=24$ female $)$, and $3(\mathrm{n}=0$ female $)$ cadets who experienced one, two, or 
three concussions respectively during the study period. There were 33 cadets $(n=11$ female) that had a repeat concussion within the same academic year (66 injuries total injuries). Of the sameseason injuries, three occurred within fourteen days of the first injury, and of these, two occurred within seven days of the first injury.

Among all concussions, injuries occurred during the following activities: $24.31 \%(\mathrm{n}=$ $175)$ varsity sports, $17.66 \%(\mathrm{n}=127)$ club sports, $6.12 \%(\mathrm{n}=44)$ intramural sports, $22.81 \%(\mathrm{n}=$ 164) physical education class, $12.52 \%(\mathrm{n}=90)$ academy training, and $16.55 \%(\mathrm{n}=119)$ during free time (Figure 3.2.1A). Injury activity across sexes are presented in Figure 3.2.1B.

Figure 3.2.1. Frequency of Injury Types

A. Injury Frequency by Activity
\[ (\mathrm{n}=800) \]

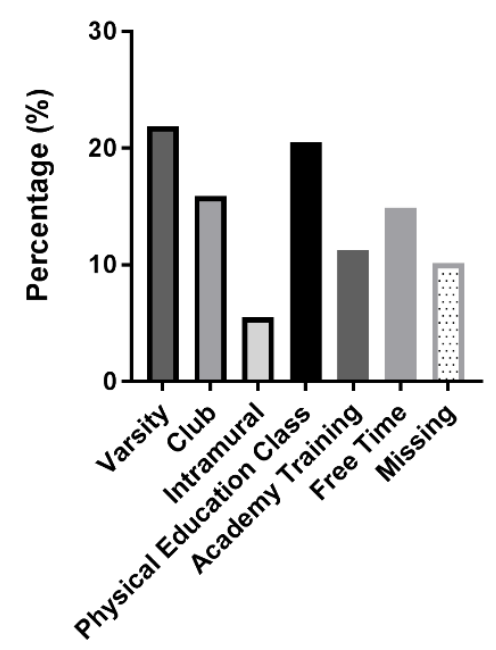

B. Injury Type by Sex
$(\mathrm{n}=\mathbf{8 0 0 )}$
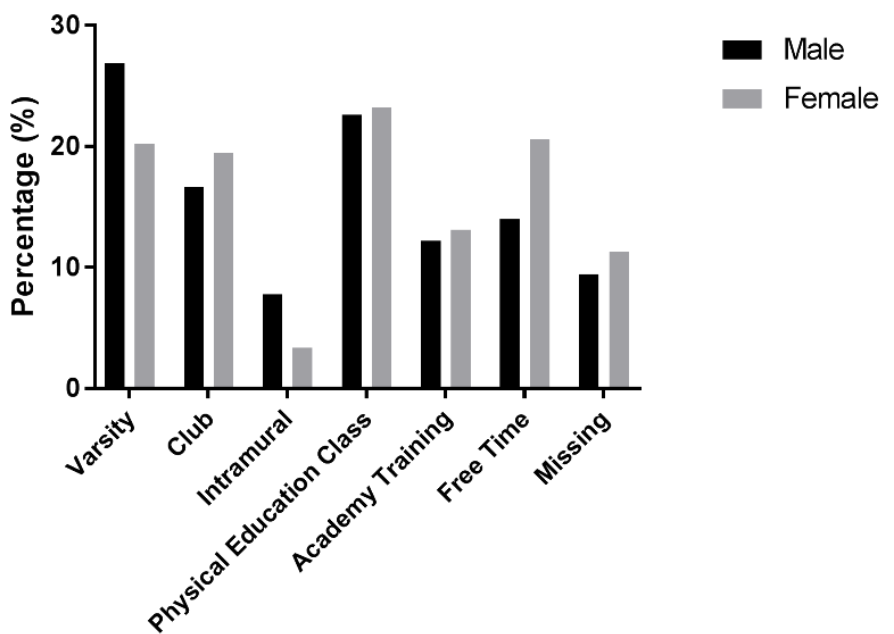

\subsubsection{Concussion Risk}

Univariate Assessment of Concussion Risk

Univariate concussion risk factors were estimated for any concussion, sport-related, training-related, and free-time related concussions. For any incident concussion, sport level, sex, freshmen status, previous concussion, headache, diagnosed migraine, diagnosed ADD/ADHD, diagnosed depression, SCAT symptoms, and BSI somatization score were all significantly associated with any incident concussion (all p's $<0.01$; Table 3.2.1a). However, only freshman 
status had a medium or larger effect size. Freshmen had over three times the risk of any concussion compared to non-freshmen (OR =3.05; 95\% CI: 2.62-3.56).

Table 3.2.1a. Univariate Associations with Any Concussion

\section{Concussion}

Sport Level

$\begin{array}{rll}\text { Varsity } & 9.14 \%(268) & 90.86 \%(2655) \\ \text { Club } & 7.09 \%(132) & 92.91 \%(1713) \\ \text { Intramural } & 5.65 \%(301) & 94.35 \%(5030)\end{array}$

Sex

$\begin{array}{ccc}\text { Female } & 11.30 \%(227) & 88.70 \%(2244) \\ \text { Male } & 5.84 \%(460) & 94.16 \%(7619)\end{array}$

Freshman

$\begin{array}{lll}\text { Yes } & 13.38 \%(369) & 86.62 \%(2388) \\ \text { No } & 4.82 \%(355) & 94.18 \%(7011)\end{array}$

Site

$\begin{array}{lll}1 & 6.15 \%(280) & 93.85 \%(4301) \\ 2 & 9.00 \%(421) & 91.00 \%(4258) \\ 3 & 2.71 \%(25) & 97.29 \%(899)\end{array}$

Previous Concussion

$\begin{array}{lll}\text { Yes } & 10.72 \%(215) & 89.28 \%(1790) \\ \text { No } & 5.92 \%(475) & 94.08 \%(7555)\end{array}$

Headaches in past 3

months

$97.29 \%(899)$
Test

$$
X^{2}(2)=35.89 ; \mathrm{p}<0.001
$$$$
\Phi=0.06
$$

Effect Size

$$
X^{2}(1)=82.89 ; \mathrm{p}<0.001
$$

OR: 2.04 (95\% CI: 1.752.39)

$\mathrm{d}=0.40$

$X^{2}(1)=83.24 ; \mathrm{p}<0.001$

OR: 3.05 (95\% CI: 2.623.56)

$d=0.62$

$$
X^{2}(2)=58.35 ; \mathrm{p}<0.001
$$$$
\Phi=0.08
$$

$$
\begin{gathered}
X^{2}(1)=57.92 ; \mathrm{p}<0.001 \\
\text { OR: } 1.91(95 \% \text { CI: } 1.61- \\
2.26)
\end{gathered}
$$$$
d=0.36
$$

$X^{2}(1)=82.04 ; \mathrm{p}<0.001$ 
No $\quad 5.67 \%(438)$

$94.33 \%$ (7285)

Diagnosed Migraine

Headache

$\begin{array}{cccc}\text { Yes } & 11.37 \%(29) & 88.63 \%(226) & X^{2}(1)=8.22 ; \mathrm{p}<0.01 \\ \text { No } & 6.77 \%(659) & 93.23 \%(9079) & \text { OR: } 1.77(95 \% \text { CI: } 1.19- \\ & & & 2.62)\end{array}$

Diagnosed

ADD/ADHD

$\begin{array}{lll}\text { Yes } & 12.95 \%(18) & 87.05 \%(130) \\ \text { No } & 6.73 \%(661) & 93.27 \%(9155)\end{array}$

Diagnosed

Depression
OR: 2.09 (95\% CI: 1.782.46)

$\mathrm{d}=0.41$ 
Sport-related concussions were significantly associated with sport level, sex, contact level, freshman status, previous concussion, headaches, and BSI somatization scores (all p's < 0.05; Table 3.2.1b). Contact sport level had the largest effect size $(\Phi=1.22)$, with all other predictors having small effect sizes. Relative to limited-contact sport cadets, contact sport cadets had 2.22 greater risk for any sport-related concussion $(\mathrm{OR}=2.22 ; 95 \% \mathrm{CI}: 1.30-4.03)$. Risk for sport-related concussion was greater for both contact $(\mathrm{OR}=5.60 ; 95 \% \mathrm{CI}: 3.11-10.92)$ and limited-contact sport cadets $(\mathrm{OR}=2.52 ; 95 \% \mathrm{CI}$ : 1.12-5.76) when compared to non-contact sport cadets.

Table 3.2.1b. Univariate Associations with Any Sport-Related Concussion

$\begin{array}{llll}\text { Concussion No Concussion } & \text { Test } & \text { Effect Size }\end{array}$

Sport Level

$\begin{array}{rll}\text { Varsity } & 5.81 \%(169) & 94.19 \%(2739) \\ \text { Club } & 4.25 \%(79) & 95.75 \%(1779) \\ \text { Intramural } & 1.23 \%(65) & 98.77 \%(5233)\end{array}$

Sex

$\begin{array}{ccccc}\text { Female } & 4.36 \%(105) & 95.64 \%(2302) & X^{2}(1)=14.00 ; \mathrm{p}<0.001\end{array} \quad \mathrm{~d}=0.25$

Contact Level^

$\begin{array}{rlr}\text { Contact } & 8.12 \%(144) & 91.88 \%(1630) \\ \text { Limited-Contact } & 3.83 \%(14) & 96.17 \%(352) \\ \text { Non-Contact } & 1.55 \%(11) & 98.45 \%(697)\end{array}$

Freshman

$\begin{array}{lcccc}\text { Yes } & 5.02 \%(137) & 94.98 \%(2594) & X^{2}(1)=40.43 ; \mathrm{p}<0.001 & \mathrm{~d}=0.40 \\ \text { No } & 2.51 \%(184) & 97.49 \%(7144) & \text { OR: } 2.05(95 \% \text { CI: } 1.64-2.57) & \end{array}$

Site

$\begin{array}{llll}1 & 3.31 \%(150) & 96.69 \%(4380) & \\ 2 & 3.45 \%(160) & 96.55 \%(4479) & X^{2}(2)=11.81 ; \mathrm{p}<0.01\end{array} \quad \Phi=0.03$


Previous

Concussion

$$
\begin{array}{lllc}
\text { Yes } & 5.39 \%(107) & 94.61 \%(1880) & X^{2}(1)=44.27 ; \mathrm{p}<0.001 \\
\text { No } & 2.50 \%(200) & 97.50 \%(7787) & \text { OR: } 2.21(95 \% \text { CI: } 1.74-2.82)
\end{array}
$$$$
\mathrm{d}=0.44
$$

Headache in the past three months

$\begin{array}{ll}\text { Yes } & 4.64 \%(103) \\ \text { No } & 2.64 \%(203)\end{array}$

$95.36 \%(2116)$

$$
X^{2}(1)=23.04 ; \mathrm{p}<0.001
$$

$97.36 \%$ (7485)

OR: 1.79 (95\% CI: 1.41-2.29)

$$
\mathrm{d}=0.32
$$

Diagnosed

Migraine

Headache

$\begin{array}{ll}\text { Yes } & 5.16 \%(13) \\ \text { No } & 3.02 \%(292)\end{array}$

$94.84 \%(239)$

$$
X^{2}(1)=3.79 ; \mathrm{p}=0.05
$$

96.98\% (9387)

OR: 1.75 (95\% CI: 0.99-3.09)

Diagnosed

ADD/ADHD

$\begin{array}{cc}\text { Yes } & 3.65 \%(5) \\ \text { No } & 3.06 \%(299)\end{array}$

$96.35 \%(132)$

$$
X^{2}(1)=0.16 ; \mathrm{p}=0.62
$$

96.94\% (9466)

OR: 1.20 (95\% CI: 0.49-2.95)

Diagnosed

Depression

$\begin{array}{cc}\text { Yes } & 3.88 \%(4) \\ \text { No } & 3.07 \%(302)\end{array}$

$96.12 \%(99)$

$$
X^{2}(1)=0.22 ; \mathrm{p}=0.56
$$

96.93\% (9528)

OR: 1.27 (95\% CI: 0.47-3.49)

Brief Sensation

Seeking Scale

$3.38(0.69)$

$3.40(0.68)$

$$
\mathrm{t}(9478)=0.48 ; \mathrm{p}=0.63
$$

SCAT Symptoms

$\begin{array}{rcccc}\text { Number } & 2.00[0.00-5.00] & 1.00[0.00-4.00] & \mathrm{Z}=3.19 ; \mathrm{p}<0.001^{\ddagger} & \mathrm{d}=0.06 \\ \text { Severity } & 2.00[0.00-9.00] & 2.00[0.00-6.00] & \mathrm{Z}=3.46 ; \mathrm{p}<0.001^{¥} & \mathrm{~d}=0.07 \\ \text { BSI Total } & 36.00[36.00-47.00] & 36.00[36.00-45.00] & \mathrm{Z}=2.24 ; \mathrm{p}=0.02^{¥} & \mathrm{~d}=0.05 \\ \text { Somatization } & 42.00[42.00-48.00] & 42.00[42.00-42.00] & \mathrm{Z}=2.73 ; \mathrm{p}<0.01^{¥} & \mathrm{~d}=0.06 \\ \text { Depression } & 42.00[42.00-45.00] & 42.00[42.00-45.00] & \mathrm{Z}=-0.38 ; \mathrm{p}=0.70^{¥} & \\ \text { Anxiety } & 39.00[39.00-47.00] & 39.00[39.00-39.00] & \mathrm{Z}=0.58 ; \mathrm{p}=0.56^{\ddagger} & \end{array}$

$\uparrow$ Satterthwaite independent t-test

$¥$ Wilcoxon two-sample test, medians and interquartile range reported 


$$
\begin{aligned}
& { }^{\wedge} \text { Only varsity cadets } \\
& * * * \mathrm{p}<0.001, * * \mathrm{p}<0.01, * \mathrm{p}<0.05
\end{aligned}
$$

Training-related concussions were associated with sport level, sex, freshmen status, previous concussion, headache, diagnosed ADD/ADHD, diagnosed depression, SCAT symptoms, and all BSI symptom (all $\mathrm{p}<0.05$; Table 3.2.1c). Freshman status and medical comorbidities (headache, diagnosed ADD/ADHD, and diagnosed depression) had medium to large effect sizes. Freshman had more than a nine-fold increased risk for incident training-related concussion relative to non-freshmen $(\mathrm{OR}=9.11 ; 95 \% \mathrm{CI}: 6.76-12.27)$. Cadets who reported having headaches in the three months before their baseline had 2.70 times increased trainingrelated concussion risk than those who did not have headaches (OR = 2.70; 95\% CI: 2.08-3.51). Similarly, cadets with diagnosed ADD/ADHD or depression had increased risk of trainingrelated concussions (Table 3.2.1c).

Table 3.2.1c. Univariate Association with Any Training-Related Concussion

\section{Concussion}

Sport Level

$\begin{array}{rrr}\text { Varsity } & 1.82 \%(53) & 98.18 \%(2855) \\ \text { Club } & 1.99 \%(37) & 98.01 \%(1821) \\ \text { Intramural } & 2.85 \%(151) & 97.15 \%(5147)\end{array}$

Sex

$\begin{array}{ccc}\text { Female } & 3.91 \%(94) & 96.09 \%(2313) \\ \text { Male } & 1.95 \%(150) & 98.05 \%(7530)\end{array}$

Freshman

$98.05 \%(7530)$
Test

Effect Size

$$
X^{2}(2)=10.07 ; \mathrm{p}<0.01
$$$$
\Phi=0.03
$$$$
\Phi=0.03
$$

$$
\begin{aligned}
& X^{2}(1)=29.59 ; \mathrm{p}<0.001 \\
& \text { OR: } 2.04(95 \% \text { CI: } 1.57-\quad \mathrm{d}=0.39 \\
& 2.65)
\end{aligned}
$$

$X^{2}(1)=302.06 ; \mathrm{p}<0.001$

OR: 9.11 (95\% CI: 6.76-

$\mathrm{d}=1.22$ 
Site

$$
\begin{array}{ccc}
1 & 1.66 \%(75) & 98.34 \%(4455) \\
2 & 3.60 \%(167) & 96.40 \%(4472) \\
3 & 0.22 \%(2) & 99.78 \%(918)
\end{array}
$$

Previous Concussion

$\begin{array}{lcccc}\text { Yes } & 3.37 \%(67) & 96.63 \%(1920) & X^{2}(1)=1035 ; \mathrm{p}<0.01 & \\ & & & \text { OR: } 1.59(95 \% \text { CI: } 1.20- & \mathrm{d}=0.26 \\ \text { No } & 2.14 \%(171) & 97.86 \%(7816) & 2.12) & \end{array}$

Headache in the past three months

$\begin{array}{ccccc}\text { Yes } & 4.64 \%(103) & 95.36 \%(2116) & X^{2}(1)=60.36 ; \mathrm{p}<0.001 & \\ & & & \text { OR: } 2.70(95 \% \text { CI: } 2.08- & \mathrm{d}=0.55 \\ \text { No } & 1.77 \%(103) & 98.23 \%(7552) & 3.51) & \end{array}$

Diagnosed Migraine Headache

$$
\begin{array}{lccc}
\text { Yes } & 3.97 \%(10) & 96.03 \%(242) & X^{2}(1)=2.68 ; \mathrm{p}=0.10 \\
\text { No } & 2.37 \%(229) & 97.63 \%(9450) & \text { OR: } 1.71(95 \% \text { CI: } 0.89-
\end{array}
$$

Diagnosed ADD/ADHD

$\begin{array}{ccccc}\text { Yes } & 7.30 \%(10) & 92.70(127) & X^{2}(1)=13.96 ; p<0.01 & \\ & & & \text { OR: } 3.26(95 \% \text { CI: } 1.69- & \mathrm{d}=0.65 \\ \text { No } & 2.36 \%(230) & 97.64 \%(9535) & 6.30) & \end{array}$

Diagnosed Depression

$\begin{array}{ccccc}\text { Yes } & 5.83 \%(6) & 94.17 \%(97) & X^{2}(1)=5.13 ; p=0.04 & \\ & & & \text { OR: } 2.53(95 \% \text { CI: } 1.10- & \mathrm{d}=0.51 \\ \text { No } & 2.38 \%(234) & 97.62 \%(9596) & 5.84) & \end{array}$

Brief Sensation

Seeking Scale

$3.35(0.57)$

$3.40(0.69)$

$\mathrm{t}(252.31)=1.48 ; \mathrm{p}=0.14^{\dagger}$

SCAT Symptoms

$$
\text { Number } \quad 4.00[1.00-9.50] \quad 1.00[0.00-4.00] \quad Z=10.16 ; p<0.001^{¥} \quad d=0.20
$$




\begin{tabular}{|c|c|c|c|c|}
\hline Severity & $\begin{array}{c}7.00[1.50- \\
18.00]\end{array}$ & $1.00[0.00-5.00]$ & $\mathrm{Z}=10.42 ; \mathrm{p}<0.001^{¥}$ & $\mathrm{~d}=0.21$ \\
\hline BSI Total & $\begin{array}{c}45.00[36.00- \\
52.00]\end{array}$ & $45.00[36.00-52.00]$ & $\mathrm{Z}=8.17 ; \mathrm{p}<0.001^{¥}$ & $d=0.16$ \\
\hline Somatization & $\begin{array}{c}48.00[42.00- \\
58.00]\end{array}$ & $48.00[42.00-58.00]$ & $\mathrm{Z}=7.97 ; \mathrm{p}<0.001^{¥}$ & $\mathrm{~d}=0.16$ \\
\hline Depression & $\begin{array}{c}42.00[42.00- \\
48.00]\end{array}$ & $42.00[42.00-48.00]$ & $\mathrm{Z}=3.98 ; \mathrm{p}<0.001^{\ddagger}$ & $\mathrm{d}=0.08$ \\
\hline Anxiety & $\begin{array}{c}39.00[39.00- \\
48.00]\end{array}$ & $39.00[39.00-48.00]$ & $\mathrm{Z}=4.67 ; \mathrm{p}<0.001^{¥}$ & $\mathrm{~d}=0.09$ \\
\hline
\end{tabular}

$\dagger$ Satterthwaite independent t-test

$¥$ Wilcoxon two-sample test, medians and interquartile range reported

$* * * \mathrm{p}<0.001, * * \mathrm{p}<0.01, * \mathrm{p}<0.05$

Sex, previous concussion, and BSI depression scores were significantly associated with free-time concussions (all p's < 0.05; Table 3.2.1d). However, only sex had at least a medium effect size. Females had 2.82 time increased risk for free-time concussions compared to males $(\mathrm{OR}=2.82 ; 95 \% \mathrm{CI}: 1.95-4.07)$.

Table 3.2.1d. Univariate Association with Free Time-Related Concussions

$\begin{array}{llcc}\text { Concussion } & \text { No Concussion } & \text { Test } & \text { Effect } \\ \text { Size }\end{array}$

Sport Level

$\begin{array}{rll}\text { Varsity } & 0.96 \%(28) & 99.04 \%(2880) \\ \text { Club } & 0.97 \%(18) & 99.03 \%(1840)\end{array} \quad X^{2}(2)=0.41 ; \mathrm{p}=0.81$

Sex

\begin{tabular}{|c|c|c|c|c|}
\hline Female & $2.24 \%(54)$ & $97.76 \%(2353)$ & $X^{2}(1)=33.25 ; p<0.001$ & \\
\hline Male & $0.81 \%(62)$ & $99.19 \%(7618)$ & $\begin{array}{c}\text { OR: } 2.82 \text { (95\% CI: } 1.95- \\
4.07)\end{array}$ & $d=0.57$ \\
\hline
\end{tabular}


Freshman

$\begin{array}{cccc}\text { Yes } & 1.43 \%(39) & 98.57 \%(2692) & X^{2}(1)=2.48 ; \mathrm{p}=0.12 \\ \text { No } & 1.05 \%(39) & 98.95 \%(7251) & \text { OR: } 1.36(95 \% \text { CI: } 0.92- \\ & & & 2.01)\end{array}$

Site

$\begin{array}{llll}1 & 0.95 \%(43) & 99.05 \%(4487) \\ 2 & 1.40 \%(65) & 98.60 \%(4574) & X^{2}(2)=4.82 ; \mathrm{p}=0.09 \\ 3 & 0.87 \%(8) & 99.13 \%(912) & \end{array}$

Previous

Concussion

$$
\text { Yes }
$$$$
1.46 \%(29)
$$

$98.54 \%$ (1958)

$$
X^{2}(1)=4.18 ; \mathrm{p}=0.04
$$

$0.94 \%(75)$

$99.06 \%$ (7912)
OR: 1.56 (95\% CI: 1.01- $\quad \mathrm{d}=0.25$ 2.41)

Headache in the past three months

Yes

$1.31 \%(29)$

$98.69 \%$ (2190)

$X^{2}(1)=1.98 ; \mathrm{p}=0.16$

No

$0.96 \%(74)$

$99.04 \%$ (7614)

OR: 1.36 (95\% CI: 0.882.10)

Diagnosed Migraine

Headache

Yes

$1.98 \%(5)$

98.02\% (247)

$X^{2}(1)=2.33 ; \mathrm{p}=0.19$

No $\quad 1.00 \%(97)$

$99.00 \%$ (9582)

OR: 1.99 (95\% CI: 0.814.96)

Diagnosed ADD/ADHD

$\begin{array}{lll}\text { Yes } & 1.46 \%(2) & 98.54 \%(135) \\ \text { No } & 1.02 \%(100) & 98.98 \%(9655)\end{array}$

$$
X^{2}(1)=0.25 ; \mathrm{p}=0.65
$$

OR: 1.43 (95\% CI: 0.355.87)

Diagnosed

Depression

$\begin{array}{ll}\text { Yes } & 2.91 \%(3) \\ \text { No } & 1.01 \%(99)\end{array}$

$97.09 \%(100)$

$X^{2}(1)=3.64 ; \mathrm{p}=0.09$

$98.99 \%(9731)$

OR: 2.95 (95\% CI: 0.929.46) 


\begin{tabular}{|c|c|c|c|c|}
\hline $\begin{array}{l}\text { Brief Sensation } \\
\text { Seeking Scale }\end{array}$ & $3.46(0.76)$ & $3.40(0.68)$ & $\mathrm{t}(9478)=-0.84 ; \mathrm{p}=0.42$ & \\
\hline \multicolumn{5}{|l|}{ SCAT Symptoms } \\
\hline Number & $2.00[0.00-5.00]$ & $1.00[0.00-4.00]$ & $\mathrm{Z}=1.95 ; \mathrm{p}=0.5^{¥}$ & \\
\hline Severity & $3.00[0.00-7.00]$ & $2.00[0.00-6.00]$ & $\mathrm{Z}=2.04 ; \mathrm{p}=0.04^{\ddagger}$ & $\mathrm{d}=0.04$ \\
\hline BSI Total & $36.00[36.00-45.00]$ & $36.00[36.00-45.00]$ & $Z=-0.79 ; p=0.43^{\#}$ & \\
\hline Somatization & $42.00[41.00-48.00]$ & $42.00[42.00-42.00]$ & $\mathrm{Z}=-1.28 ; \mathrm{p}=0.20^{¥}$ & \\
\hline Depression & $42.00[40.00-45.00]$ & $42.00[42.00-45.00]$ & $Z=-2.04 ; p=0.04^{\ddagger}$ & $\mathrm{d}=0.04$ \\
\hline Anxiety & $39.00[38.00-45.00]$ & $39.00[39.00-39.00]$ & $Z=-1.97 ; p=0.05^{¥}$ & \\
\hline
\end{tabular}

$\dagger$ Satterthwaite independent t-test

$¥$ Wilcoxon two-sample test, medians and interquartile range reported

$* * * \mathrm{p}<0.001, * * \mathrm{p}<0.01, * \mathrm{p}<0.05$

Univaraiate tests evaluated sex as a possible moderator. Thus, concussion predictors were assessed for their association with sex (Supplementary Tables Appendix A). All variables were significantly associated with sex (all p's $<0.05$ ). The largest effect sizes were observed with sport level, contact level among varsity cadets, diagnosed depression, and BSI scores.

Interactions between sex and these variables were assessed in the mixed models.

Mixed Model Results - All Cadets

Service Academy was a significant random effect for each mixed model. Each

subsequent model includes site as a random effect. All models are included in the supplementary materials (Appendix A). The final models are interpreted below.

Females, varsity and club cadets, freshmen were cadet characteristics associated with increased risk for any concussion (Table 3.2.2a). Additionally, medical history including previous concussion, headache, and diagnosed ADD/ADHD all increased concussion risk. Finally, increasing BSI somatization scores were associated with increased concussion risk (Table 3.2.2a). Each factor was significant after controlling for all other covariates. There were no significant interactions between sport level, depression or BSI symptom scores with sex (all p $>0.05)$. 
Table 3.2.2a. Mixed Model Results - Any Concussion

\begin{tabular}{|c|c|c|c|c|c|c|c|c|}
\hline Parameter & Level & Estimate & Standard Error & t Value & $\operatorname{Pr}>|t|$ & Odds Ratio & Lower & Upper \\
\hline Intercept & & -5.09 & 0.58 & -8.80 & 0.01 & & & \\
\hline \multirow[t]{2}{*}{ Sex } & Female & 0.70 & 0.09 & 8.01 & $<.0001$ & 2.02 & 1.70 & 2.40 \\
\hline & Male & Ref. & & & & & & \\
\hline \multirow[t]{3}{*}{ Sport Level } & Varsity & 0.48 & 0.10 & 5.02 & $<.0001$ & 1.61 & 1.34 & 1.95 \\
\hline & Club & 0.31 & 0.11 & 2.71 & 0.01 & 1.36 & 1.09 & 1.70 \\
\hline & Intramural & Ref. & & & & & & \\
\hline \multirow[t]{2}{*}{ Freshmen } & Yes & 1.00 & 0.09 & 11.10 & $<.0001$ & 2.73 & 2.29 & 3.26 \\
\hline & No & Ref. & & & & & & \\
\hline \multirow[t]{2}{*}{ Previous Concussion } & Yes & 0.68 & 0.09 & 7.40 & $<.0001$ & 1.98 & 1.65 & 2.37 \\
\hline & No & Ref. & & & & & & \\
\hline \multirow[t]{2}{*}{$\begin{array}{l}\text { Headache in the past three } \\
\text { months }\end{array}$} & Yes & 0.40 & 0.09 & 4.37 & $<.0001$ & 1.50 & 1.25 & 1.80 \\
\hline & No & Ref. & & & & & & \\
\hline \multirow[t]{2}{*}{ ADD/ADHD } & Yes & 0.73 & 0.27 & 2.73 & 0.01 & 2.08 & 1.23 & 3.52 \\
\hline & No & Ref. & & & & & & \\
\hline BSI Somatization & & 0.02 & 0.01 & 3.36 & 0.00 & 1.02 & 1.01 & 1.03 \\
\hline
\end{tabular}

Odds ratios for categorical predictors estimated at BSI somatization mean (44.57) 
Similar to all concussion risk, females, varsity and club cadets, and freshmen status were characteristics associated with increased risk for sport-related concussion (Table 3.2.2b). Varsity cadets had the greatest risk for sport-related concussion compared to club $(\mathrm{OR}=1.38$; $95 \% \mathrm{CI}$ : 1.03-1.83) and intramural cadets ( $\mathrm{OR}=5.33$; 95\% CI: 3.93-7.22). Previous concussion and headache were associated with two and 1.5 times greater risk for sport concussion (Table 3.2.2b). Finally, increasing BSI somatization symptoms were associated with increased sport-related concussion risk (Table 3.2.2b). 
Table 3.2.2b. Mixed Model Results - Sport-Related Concussion

\begin{tabular}{|c|c|c|c|c|c|c|c|c|}
\hline Parameter & Level & Estimate & Standard Error & t Value & $\operatorname{Pr}>|t|$ & Odds Ratio & Lower & Upper \\
\hline Intercept & & -6.54 & 0.69 & -9.46 & 0.01 & & & \\
\hline \multirow[t]{2}{*}{ Sex } & Female & 0.32 & 0.13 & 2.45 & 0.01 & 1.38 & 1.07 & 1.78 \\
\hline & Male & Ref. & & & & & & \\
\hline \multirow[t]{3}{*}{ Sport Level } & Varsity & 1.67 & 0.16 & 10.78 & $<.0001$ & 5.33 & 3.93 & 7.22 \\
\hline & Club & 1.35 & 0.17 & 7.75 & $<.0001$ & 3.87 & 2.75 & 5.45 \\
\hline & Intramural & Ref. & & & & & & \\
\hline \multirow[t]{2}{*}{ Freshmen } & Yes & 0.47 & 0.13 & 3.57 & 0.00 & 1.60 & 1.24 & 2.06 \\
\hline & No & Ref. & & & & & & \\
\hline \multirow[t]{2}{*}{ Previous Concussion } & Yes & 0.70 & 0.13 & 5.44 & $<.0001$ & 2.01 & 1.56 & 2.58 \\
\hline & No & Ref. & & & & & & \\
\hline \multirow[t]{2}{*}{$\begin{array}{l}\text { Headache in the past } \\
\text { three months }\end{array}$} & Yes & 0.43 & 0.14 & 3.19 & 0.00 & 1.54 & 1.18 & 2.01 \\
\hline & No & Ref. & & & & & & \\
\hline BSI Somatization & & 0.02 & 0.01 & 2.69 & 0.01 & 1.03 & 1.01 & 1.04 \\
\hline
\end{tabular}

Odds ratios estimated at mean BSI Somatization symptom score (44.58)

Effect of BSI Somatization symptom score is assessed as one unit offsets from the mean. 
Among training related concussions, sex significantly interacted with BSI somatization symptoms. With increasing BSI somatization symptoms, the risk for training-related concussion increased, but at a greater rate for males compared to females (Table 3.2.2c). Varsity cadets had a 44\% lower risk for training-related concussions compared to intramural cadets $(\mathrm{OR}=0.56$; 95\% CI: 0.40-0.78). There were no significant differences between varsity and club, or club and intramural cadets. Freshmen had eight times greater risk for a training related concussion compared to non-freshmen (OR $=8.17$; 95\% CI: 5.87-11.37). Previous concussion, headache, and diagnosed ADD/ADHD also significantly increased the risk of a training-related concussion (Table 3.2.2c). 
Table 3.2.2c. Mixed Model Results - Academy Training-Related Concussion

\begin{tabular}{|c|c|c|c|c|c|c|c|c|}
\hline Parameter & Level & Estimate & $\begin{array}{l}\text { Standard } \\
\text { Error }\end{array}$ & t Value & $\operatorname{Pr}>|t|$ & $\begin{array}{l}\text { Odds } \\
\text { Ratio }\end{array}$ & Lower & Upper \\
\hline Intercept & & -7.81 & 0.98 & -8.00 & 0.02 & & & \\
\hline \multirow[t]{2}{*}{ Sex } & Female & 2.69 & 0.79 & 3.43 & 0.00 & 2.32 & 1.70 & 3.18 \\
\hline & Male & Ref. & & & & & & \\
\hline \multirow[t]{3}{*}{ Sport Level } & Varsity & -0.59 & 0.17 & -3.44 & 0.00 & 0.56 & 0.40 & 0.78 \\
\hline & Club & -0.28 & 0.20 & -1.42 & 0.15 & 0.76 & 0.52 & 1.11 \\
\hline & Intramurals & Ref. & & & & & & \\
\hline \multirow[t]{2}{*}{ Freshmen } & Yes & 2.10 & 0.17 & 12.47 & $<.0001$ & 8.17 & 5.87 & 11.37 \\
\hline & No & Ref. & & & & & & \\
\hline \multirow[t]{2}{*}{ Previous Concussion } & Yes & 0.55 & 0.16 & 3.49 & 0.00 & 1.73 & 1.27 & 2.36 \\
\hline & No & Ref. & & & & & & \\
\hline \multirow[t]{2}{*}{$\begin{array}{l}\text { Headache in the past three } \\
\text { months }\end{array}$} & Yes & 0.42 & 0.15 & 2.88 & 0.00 & 1.53 & 1.15 & 2.04 \\
\hline & No & Ref. & & & & & & \\
\hline \multirow[t]{2}{*}{$\mathrm{ADD} / \mathrm{ADHD}$} & Yes & 1.27 & 0.37 & 3.44 & 0.00 & 3.55 & 1.73 & 7.32 \\
\hline & No & Ref. & & & & & & \\
\hline BSI Somatization & & 0.04 & 0.01 & 3.81 & 0.00 & 1.04 & 1.02 & 1.07 \\
\hline Sex*BSI Somatization & Female & -0.04 & 0.02 & -2.64 & 0.01 & 0.96 & 0.93 & 0.99 \\
\hline
\end{tabular}

Odds ratios estimated at mean BSI Somatization symptom score (44.57)

Effect of BSI Somatization symptom score is assessed as one unit offsets from the mean. 
Risk for free time-related concussions was significantly estimated by sex, freshmen status, and previous concussion. Females, freshmen, and cadets with a prior concussion were all at significantly increased risk for a free time-related concussion (Table 3.2.2d).

Table 3.2.2d. Mixed Model Results - Free Time-Related Concussion

\begin{tabular}{lcccccccc}
\hline Parameter & Level & Estimate & $\begin{array}{c}\text { Standard } \\
\text { Error }\end{array}$ & t Value & Pr $>|\mathbf{t}|$ & $\begin{array}{c}\text { Odds } \\
\text { Ratio }\end{array}$ & Lower & Upper \\
\hline Intercept & & -5.2944 & 0.2582 & -20.51 & 0.0024 & & & \\
Sex & Female & 1.1167 & 0.201 & 5.56 & $<.0001$ & 3.055 & 2.06 & 4.529 \\
& Male & Ref. & & & & & & \\
Freshmen & Yes & 0.4267 & 0.207 & 2.06 & 0.0393 & 1.532 & 1.021 & 2.299 \\
& No & Ref. & & & & & & \\
Previous & Yes & 0.5516 & 0.2221 & 2.48 & 0.013 & 1.736 & 1.123 & 2.683 \\
Concussion & No & Ref. & & & & & & \\
\end{tabular}

\section{Mixed Model Results - Varsity Cadets}

Within varsity athlete cadets there was a significant sex and BSI anxiety interaction. With increasing BSI anxiety symptom scores, concussion risk increased at a greater rate for males than females (Table 3.2.2e). Contact sport cadets were at greater risk for any concussion than noncontact cadets $(\mathrm{OR}=2.43 ; 95 \% \mathrm{CI}: 1.60-3.68)$. There was no significant difference in risk between contact and limited-contact or limited and non-contact cadets (both p's $\geq 0.05$ ). As observed within the entire cadet sample, varsity females, freshmen, cadets with a previous concussion, or headache were all at increased risk for concussion (Table 3.2.2e). 
Table 3.2.2e. Mixed Model Results - Any Concussion among Varsity Athlete Cadets

\begin{tabular}{|c|c|c|c|c|c|c|c|c|c|}
\hline Parameter & Level & Estimate & $\begin{array}{l}\text { Standard } \\
\text { Error }\end{array}$ & DF & t Value & $\operatorname{Pr}>|t|$ & $\begin{array}{l}\text { Odds } \\
\text { Ratio }\end{array}$ & Lower & Upper \\
\hline Intercept & & -5.36 & 0.80 & 2.00 & -6.70 & 0.02 & & & \\
\hline \multirow[t]{2}{*}{ Sex } & Female & 3.03 & 0.93 & 2696.00 & 3.25 & 0.00 & 2.11 & 1.56 & 2.86 \\
\hline & Male & Ref. & & & & & & & \\
\hline \multirow[t]{3}{*}{ Contact Level } & Contact & 0.89 & 0.21 & 2696.00 & 4.18 & $<.0001$ & 2.43 & 1.60 & 3.68 \\
\hline & Limited-Contact & 0.54 & 0.27 & 2696.00 & 1.97 & 0.05 & 1.72 & 1.00 & 2.93 \\
\hline & Non-Contact & Ref. & & & & & & & \\
\hline \multirow[t]{2}{*}{ Freshmen } & Yes & 0.77 & 0.15 & 2696.00 & 5.15 & $<.0001$ & 2.15 & 1.61 & 2.88 \\
\hline & No & Ref. & & & & & & & \\
\hline \multirow[t]{2}{*}{ Previous Concussion } & Yes & 0.58 & 0.15 & 2696.00 & 3.88 & 0.00 & 1.79 & 1.33 & 2.40 \\
\hline & No & Ref. & & & & & & & \\
\hline \multirow[t]{2}{*}{$\begin{array}{l}\text { Headache in the past three } \\
\text { months }\end{array}$} & Yes & 0.46 & 0.16 & 2696.00 & 2.88 & 0.00 & 1.58 & 1.16 & 2.16 \\
\hline & No & Ref. & & & & & & & \\
\hline BSI Anxiety & & 0.03 & 0.01 & 2696.00 & 2.04 & 0.04 & 1.03 & 1.00 & 1.05 \\
\hline Sex*BSI Anxiety & Female & -0.05 & 0.02 & 2696.00 & -2.49 & 0.01 & 0.95 & 0.91 & 0.99 \\
\hline
\end{tabular}

Odds ratios for categorical predictors estimated at mean of BSI Anxiety score (41.85)

Effect of BSI Anxiety score is assessed as one unit offsets from the mean. 
Within varsity-sport injuries, females had 1.71 times the risk for a varsity sport concussion compared to males (Table 3.2.2f). Contact and limited-contact sport cadets had a greater risk of a sport-related concussion than non-contact sport cadets. Contact sport cadets had 2.15 times greater risk than limited contact cadets for sport-related concussion (95\% CI: 1.183.92). Previous concussion and increasing BSI somatization symptom scores also increased sport-related concussion risk (Table 3.2.2f). 
Table 3.2.2f. Mixed Model Results - Any Sport Concussion among Varsity Athletes

\begin{tabular}{|c|c|c|c|c|c|c|c|c|c|}
\hline Parameter & Level & Estimate & Standard & t Value & $\operatorname{Pr}>|\mathbf{t}|$ & $\begin{array}{l}\text { Odds } \\
\text { Ratio }\end{array}$ & Lower & Upper & $\begin{array}{c}\text { Effect } \\
\text { Size }\end{array}$ \\
\hline Intercept & & -7.1173 & 0.9381 & -7.59 & 0.0169 & & & & \\
\hline & Male & Ref. & & & & & & & \\
\hline \multirow[t]{2}{*}{ Contact Level } & Contact & 2.0257 & 0.3986 & 5.08 & $<.0001$ & 7.581 & 3.47 & 16.565 & 1.119141 \\
\hline & Non-Contact & Ref. & & & & & & & \\
\hline \multirow[t]{2}{*}{ Previous Concussion } & Yes & 0.5706 & 0.1773 & 3.22 & 0.0013 & 1.769 & 1.25 & 2.505 & 0.315146 \\
\hline & No & Ref. & & & & & & & \\
\hline BSI Somatization & & 0.04098 & 0.01217 & 3.37 & 0.0008 & 1.042 & 1.017 & 1.067 & 0.02273 \\
\hline
\end{tabular}

Odds ratios for categorical predictors estimated at mean of BSI Somatization score (45.07)

Effect of BSI Somatization score is assessed as one unit offsets from the mean. 
Varsity cadets who were female $(\mathrm{OR}=1.96 ; 95 \% \mathrm{CI}$ : 1.08-3.56), freshmen $(\mathrm{OR}=15.82$; 95\% CI: 6.70-37.38), and who had a previous concussion (OR = 1.93; 95\% CI: 1.06-3.16) had increased risk for an academy training-related concussion. For free time-related concussions, sex was the only significant predictor. Females had 2.89 (95\% CI: 1.35-6.19) times greater risk for a free time-related concussion than males.

\subsubsection{Boxing Injuries}

Following the introduction of boxing with females, 15 boxing-related injuries occurred, resulting in a significant increase from 2015-2016 $(\mathrm{n}=0)$ to 2016-2017 $(\mathrm{n}=15)$. There was no effect of year among male cadets $(\mathrm{p}>0.05)$. However, there was no effect on overall concussion risk when assessing sex by year interaction. Thus, while boxing injuries increased the risk for academy training-related injuries among females, there was no significant increase overall concussion risk by introducing boxing.

Relative to other activities, boxing poses a similar concussion risk to a varsity sport, club sport, and free time-related activities (all p's > 0.05) (Figure 3.2.2). There was a trend for boxing to have a lower risk of concussion compared to varsity sport (OR: 0.75 95\% CI: 0.53-1.04).

Boxing had 63\% reduced concussion risk compared to any sport (OR: 0.37 95\% CI: 0.28-0.50) (Figure 3.2.2). Boxing risk was only significantly greater compared to intramural sport (OR: 2.81 95\% CI: 1.71-4.61) (Figure 3.2.2). 
Figure 3.2.2. Concussion Risk by Activity

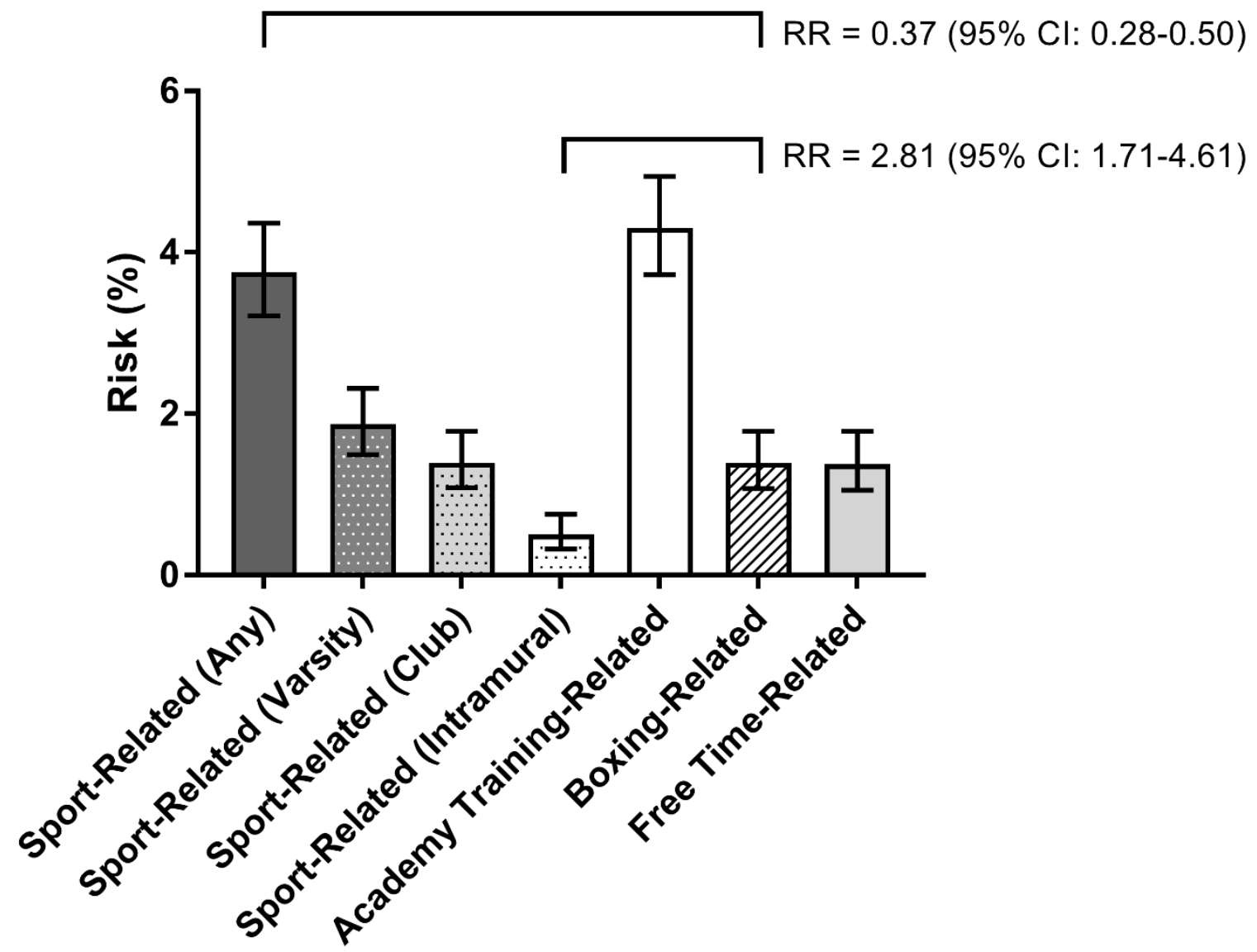

\subsubsection{Concussion Rates}

Concussion rates are presented in Table 3.2 .3 by sex and sport level for each concussion type. All rates are estimated per 100 cadet years.

\section{All Cadets}

The cadet concussion rate was 4.62 concussions per 100 cadet-years. Females had over twice the concussion rate as males (Rate Ratio $(\mathrm{RR})=2.04$; 95\% CI: 1.75-2.38) (Table 3.2.3). Varsity cadets sustained concussions at 1.75 (95\% CI: 1.48-2.07) times greater rate than club cadets and 1.41 (95\% CI: 1.14-1.75) times greater than intramural cadets (Table 3.2.3). Club cadets also sustained concussions at a higher rate than intramural cadets $(\mathrm{RR}=1.24 ; 95 \% \mathrm{CI}$ : 1.01-1.52). 
Females sustained sport related concussions (RR: 1.63 95\% CI: 1.28-2.08), academy training-related (RR: 2.17 95\% CI: 1.66-2.85), and free time-related concussions (Rate Ratio: 3.02 95\% CI: 2.03-4.52) at a significantly higher rate than males (Table 3.2.3). While varsity (RR: 5.33; 95\% CI: 3.96-7.17) and intramural cadets (RR: 3.44; 95\% CI: 2.44-4.85) sustained sport-related concussions at a significantly greater rate than intramural cadets, intramural cadets that had significantly greater academy training-related concussion rates than varsity (RR: 1.43 95\% CI: 1.04-1.97) or club cadets (RR: 1.52 95\% CI: 1.04-2.34) (Table 3.2.3). There were no differences in free time-related concussions across sport levels (all p's $>0.05$ ). 
Table 3.2.3. Concussion rate by sex and sport level status, per 100 cadet-years

\begin{tabular}{llcccc}
\hline & & All Concussions & $\begin{array}{c}\text { Any Sport } \\
\text { Concussions }\end{array}$ & $\begin{array}{c}\text { Academy Training } \\
\text { Concussions }\end{array}$ & $\begin{array}{c}\text { Free Time } \\
\text { Concussions }\end{array}$ \\
\hline Sex & Female & $7.62(6.74-8.61)$ & $2.81(2.30-3.44)$ & $2.58(2.09-3.18)$ & $1.33(1.00-1.79)$ \\
& Male & $3.74(3.40-4.11)$ & $1.79(1.51-1.99)$ & $1.18(1.01-1.40)$ & $0.44(0.34-0.58)$ \\
\hline Sport Level & Varsity & $6.57(5.82-7.43)$ & $4.09(3.50-4.77)$ & $1.27(0.96-1.67)$ & $0.58(0.39-0.88)$ \\
& Club & $4.65(3.90-5.54)$ & $2.63(2.09-3.33)$ & $1.19(0.84-1.68)$ & $0.63(0.39-1.02)$ \\
& Intramural & $3.76(3.35-4.21)$ & $0.77(0.59-0.99)$ & $1.82(1.54-2.13)$ & $0.70(0.54-0.92)$ \\
\hline
\end{tabular}




\subsubsection{Discussion}

The objective of the current study was to determine risk factors for concussions among United States Service Academies cadets across multiple injury domains. Different risk factors were observed for each concussion type, highlighting specific at-risk populations. Specifically, females and increasing contact level were replicated as risk factors for concussion. New findings revealed that varsity athlete cadets and increasing somatization symptoms were associated with increased concussion risk. Finally, boxing-related risk was similar to varsity athlete concussion risk.

\subsubsection{Concussion Risk Factors}

Female sex, freshmen status, previous concussion, and headache were the most consistent concussion risk factors. However, the strength of these associations varied by concussion-type. Females had the greatest risk and strongest effect for all concussion and free time-related concussions. Freshmen had the strongest effect on academy training-related injuries. Sport level had the greatest effect on sport-related concussions while contact sport level had the strongest association for varsity sport-related injuries.

Females had increased overall, sport-related, academy training-related, and free timerelated concussion risk, which is similar to previous findings within high school and collegiate athletes showing females are more likely to sustain sport-related concussions (Abrahams et al., 2014; Covassin et al., 2003; Lincoln et al., 2011). Outside of athletics, males have greater risk for military-related concussion (Hoge et al., 2008) and all traumatic brain injury (Bazarian et al., 2005; Cassidy et al., 2004). However, studies investigating concussion incidence within service members have limited their investigation to active-duty concussions and enrolled few females (Hoge et al., 2008; Wilk et al., 2010). Unlike previous studies of service members sustaining active-duty concussions (Cameron et al., 2012; Hoge et al., 2008) or emergency department admission studies (Bazarian et al., 2005; Cassidy et al., 2004), current results demonstrated that female cadets had increased risk for academy training-related and free time-related concussions. Thus, greater concussion risk among females is not limited to sports-related concussions. While previous studies have found increased active-duty concussion risk for males, these injuries are likely due to different exposure mechanisms than cadet academy training-related injuries (Cameron et al., 2012; Hoge et al., 2008). Compared to the study by Cameron and colleagues (Cameron et al., 2012), concussions in the current study were prospectively diagnosed rather 
than retrospectively compiled using ICD-9 codes. Relying on ICD-9 codes to identify concussions (Cameron et al., 2012) could result in misclassification (Bazarian et al., 2006), and subsequent bias. Additionally, only 5-12\% of the previous studies samples were female, compared to $23 \%$ in the current study. The difference in injury severity, injury identification, and limited female enrollment may explain the different results between previous studies finding higher concussion risk among males while the current study found higher concussion risk among females. Future studies should further examine this finding to determine whether civilian females also experience greater concussion risk across multiple injury domains.

In the current study, females had greater concussion risk during free time which contradicts previous studies finding males at increased risk (Bazarian et al., 2005; Cassidy et al., 2004). Males have more TBI related emergency department visits for all TBI severities (Fu, Jing, Fu, \& Cusimano, 2016). However, females may be closing the gap. Between 2002 and 2009 the male TBI emergency department visit rate decreased 9\% while female rate increased $7 \%$ (Fu et al., 2016). Earlier studies specifically examining concussion (Bazarian et al., 2005; Cassidy et al., 2004) may have captured lower health care utilization by females or lower concussion incidence by females. Alternatively, mechanisms of free time-related concussions captured in the current study may not be similar to emergency department related admissions. Almost one-third of emergency department concussion visits were due to sports-related incidents (30\%) (Gaw \& Zonfrillo, 2016). Another third were due to traffic-related head trauma (Gaw \& Zonfrillo, 2016). Differences in concussion mechanisms and motivations for utilizing health care services may contribute to the current analysis finding females at greater risk for free time-related concussions. Future studies should continue to examine sex-related differences in concussion risk resulting from mechanisms which commonly present to the emergency department (e.g. motor vehicle accidents).

While the current results conflict with studies from military and emergency department epidemiological studies of concussion, there is biologically plausible reasons for while females may be at greater concussion risk. Lower neck strength among females relative to males is proposed to reduce the bracing capacities of female athletes, increasing their risk for concussion (Eckner, Oh, Joshi, Richardson, \& Ashton-Miller, 2014; Tierney et al., 2005). Increased concussion rates may also be due to increased reporting among females. Females endorse more concussion-related symptoms(Covassin, Elbin, Harris, Parker, \& Kontos, 2012) and are more 
likely to report an injury (Wallace, Covassin, \& Beidler, 2017). Hormonal differences may also predispose females to concussions. Human studies have observed worse recovery when injured during the luteal phase of the menstrual cycle (Wunderle, Hoeger, Wasserman, \& Bazarian, 2014). Thus, it is unclear if females sustain more concussions due to physical, physiological or psychological mechanisms.

Previous concussion, headaches in the previous three months, and diagnosed ADD/ADHD disorders all increased concussion risk and replicated findings from the sports medicine literature (Abrahams et al., 2014; Kutcher \& Eckner, 2010). Furthermore, results from the current study furthered the understanding of concussion risk by demonstrating previous concussion, headache, and diagnosed ADD/ADHD disorders all occurred before the incident concussion. Establishing temporality of pre-concussion medical diagnosis provided supporting causal evidence that previous concussion, headaches, and ADD/ADHD increase concussion risk.

Pre-existing headaches and ADD/ADHD diagnoses have not been assessed for incident concussion risk among service members or cadets. Headache symptoms and ADD/ADHD diagnoses have primarily been an outcome measure after TBI (Miller et al., 2015; Wells, 2010). In the military, screening for post-deployment TBI began in 2008 and focused on a service member's most recent deployment (Iverson, Langlois, McCrea, \& Kelly, 2009). Thus, establishing a TBI history was difficult, and to our knowledge, no study has examined the relationship between previous medical history and incident concussion among male and female service members.

Increased baseline anxiety and depression symptoms were hypothesized to increase cadet's incident concussion risk. However, baseline depression symptoms did not affect subsequent concussion risk. There was a significant interaction between sex and anxiety symptoms among any concussion among varsity athlete cadets. In support of our proposed the hypothesis, concussion risk increased with increasing baseline anxiety symptom scores, which has also been linked to risk for any injury ( $\mathrm{Li}$ et al., 2017). The current study utilized the BSI-18 to assess psychological symptoms while Li and colleagues (Li et al., 2017) used the trait portion of the State-Trait Anxiety Inventory (Spielberger, 1989) and the Center for Epidemiologic Studies Depression Scale (Radloff, 1977). Additionally, rather than using the symptom scores as continuous variables, Li and colleagues ( $\mathrm{Li}$ et al., 2017) set cutoffs to categorize participants as having elevated anxiety and depression symptoms (Li et al., 2017). While anxiety symptoms 
influence both general injury risk (Li et al., 2017) and concussion risk, depression symptoms only appear to be associated with general injury risk (Li et al., 2017), not concussion risk.

Finally, compared to the previous study by Li and colleagues (Li et al., 2017), somatization symptom scores were included in the current analysis. Somatization symptoms were found to significantly estimate any concussion, sport-related concussion, and varsity sportrelated concussion risk. With increasing somatization the tendency to express psychosocial or emotional problems as physical ailments increases (American Psychiatric Association, 2013). Increased pre-concussion somatization symptom score has been shown to increase recovery time post-concussion (Nelson et al., 2016). Specifically, pre-injury somatization increased acute postconcussion symptoms, extending symptom recovery (Nelson et al., 2016). Thus, increasing baseline somatization symptoms may influence concussion risk by exacerbating symptoms, facilitating diagnosis. It is unclear whether increasing somatization directly increases concussion risk or diagnosis via increased symptom experience or reporting. Given the extremely healthy cadet population, small increases in psychological symptoms, particularly somatization, increase concussion risk. While results should be interpreted cautiously due to their small effect size, the effect of anxiety and somatization symptoms remained significant in multivariate models.

\subsubsection{Concussion Rates}

The current study's overall concussion rate was four times greater than the rate published by Cameron and colleagues (Cameron et al., 2012). However, data collection occurred between 1997-2007, for the previous study, observing a sharp increase in concussion rates between 20052007 (Cameron et al., 2012). Increasing concussion awareness and knowledge since 2007 may contribute to the differences between the current study and the previous study. Moreover, Cameron and colleagues (Cameron et al., 2012) identified concussion using ICD-9 codes which can misclassify concussions and non-concussions (Bazarian et al., 2006). While cadet concussion rates are higher than active duty service member concussion rates, differences in concussion knowledge, study methodologies, and sample characteristics likely contribute to the concussion rate difference.

Concussion rates increased with increasing competition level with varsity athlete cadets demonstrating the greatest concussion rate. Collegiate athletes have a greater concussion rate than high school athletes (Gessel et al., 2007). Moreover, male Division I ice hockey players had a greater concussion rate than male Division III players (Rosene et al., 2017). The increased 
concussion rate among varsity cadets compared to club and intramural cadets adds to the evidence for increasing level of play and increased concussion rate.

\subsubsection{Limitations}

The current study is not without limitation. Best efforts were made to collect every incident concussion. However, due to non-reporting, it is possible incident concussions were missed which may bias the results. Roughly $30 \%$ of collegiate athletes do not report their concussion (Kerr, Register-Mihalik, Kroshus, Baugh, \& Marshall, 2016; LaRoche et al., 2016), however it is unknown whether similar non-reporting rates exist among cadets. Certain cadet characteristics, for example aspiring Air Force pilots, have lower reporting behaviors (D'Lauro, Johnson, Foster, McGinty, \& Campbell, 2017). Thus, it is possible that certain cadet populations are at greater risk than as indicated in the current analysis. Thus, significant unreported concussions would underestimate the true concussion risk. However, more detailed estimates of cadet non-reporting and patterns among cadets are needed to further specify the direction of the bias.

The interpretation of concussion rates may be difficult to compare with other epidemiological studies. Exposure was estimated at the duration from enrollment to injury or end of study participation. This crude estimate does not limit exposure to the days of possible exposure. Thus, the amount of exposure may be an overestimate and subsequently underestimating the true concussion rate.

\subsubsection{Conclusion}

Concussion risk factors varied by the type of concussion, highlighting unique at-risk populations. Female sex, previous concussion, and headache were the most consistent risk factors for concussion across all settings. Previously unidentified risk factors including freshmen status and pre-injury somatization symptoms also significantly influence concussion risk among Service Academy cadets. By characterizing those at risk, better-targeted screening protocols can be developed to diagnose and treat concussion.

\section{Chapter 3.3 Project 2: Concussion Recovery Trajectories}

\subsubsection{Introduction}

Despite a steady increase in concussion-related research, the literature has yet to elucidate injury recovery. Understanding concussion's natural history enables clinicians to educate their 
patients on typical concussion recovery and identify and manage patients at risk for prolonged recovery.

Two significant milestones compose the recovery process. The first recovery milestone is comprised of the duration until symptom resolution (McCrory et al., 2017), defined using clinical judgment (Doolan et al., 2012) or when an individual returns to their pre-injury symptom norms (McCrea et al., 2013). Following symptom resolution is a second phase encapsulating the return to activity protocol (RTA) (Echemendia \& Cantu, 2003). The protocol progressively increases physical exertion over time as long as symptoms do not re-emerge. If symptoms do re-emerge, the clinician reduces the physical exertion, and the protocol extends until the individual can complete maximal intensity without symptoms (McCrory et al., 2017; Wallace, Covassin, \& Lafevor, 2016). To date, most studies have investigated factors related to total time loss (Asplund, McKeag, \& Olsen, 2004; Covassin et al., 2016; Davis-Hayes et al., 2017; Pfaller, Nelson, Apps, Walter, \& McCrea, 2016), without assessing each of the two recovery phases. Moreover, no study has systematically examined factors associated with return to play protocol duration, nor has there been an examination of individual and injury factors associated with both components of recovery.

The recent International Concussion Consensus group identified multiple pre-injury individual and acute injury characteristics associated with total recovery time (Iverson et al., 2017). Pre-injury characteristics such as age, sex, race, genetics, prior concussion, attention deficit disorder, learning disability, psychiatric history, migraine, and headache history were all identified (Iverson et al., 2017). While post-injury characteristics including loss of consciousness (LOC), post-traumatic amnesia (PTA), retrograde amnesia (RGA), delayed removal from play, greater initial symptoms, and specific symptoms (e.g. headache, dizziness, balance) were also identified as recovery factors (Iverson et al., 2017). Factors with the most substantial evidence were sex, prior concussion, headache history, LOC, PTA, RGA, delayed removal from play, acute symptom burden, and post-concussion headache (Iverson et al., 2017). However, each of these items has had mixed findings.

While informative, the Iverson review focused on sport-related concussion, limiting the application to non-sport injuries. For example, compared to sport-related concussion, individuals seeking care at the emergency department for a concussion are more likely to have nausea (Delaney, Abuzeyad, Correa, \& Foxford, 2005), loss of consciousness (Delaney et al., 2005; 
Nelson et al., 2018), ongoing litigation (Nelson et al., 2018), suffered more severe brain injury (Setnik \& Bazarian, 2007), and the most common mechanism is motor vehicle accidents (Nelson et al., 2018). Individual differences between concussion types (sport vs. non-sport) make it difficult to identify consistent factors associated with recovery.

Given the limitations of the literature to date, the goal of the current investigation is to describe and identify individual and acute injury characteristics associated with time until asymptomatic and duration of RTA protocol among a diverse cohort of United States service academy cadets after sport, academy training, and free-time related concussions. We hypothesize that greater symptom burden will increase the time until asymptomatic and completion of RTA protocol. Cadet athletic status, a varsity athlete or non-varsity athlete, will influence symptom and return to activity durations, with shorter durations associated with varsity athlete cadets due to more frequent interactions with clinical staff. Finally, we hypothesize that males will have a shorter time until asymptomatic, but we do not expect to find a significant sex effect in RTA protocol duration.

\subsubsection{Methods}

\subsubsection{Study Design}

The current study is part of a larger joint effort by the U.S. Department of Defense (DoD) and National Collegiate Athletic Association (NCAA). This partnership funded the Concussion, Assessment, Research, and Education (CARE) Consortium, a multi-site investigation on the natural history of concussion. A detailed description of the CARE organization has been published previously (Broglio, McCrea, et al., 2017).

All cadets at participating Service Academies were eligible to enroll in CARE. Each participating cadet provided written informed consent that was approved by the site-level Institutional Review Board (IRB) and DoD Human Research Protections Office (HRPO) (Broglio, McCrea, et al., 2017). Before each academic year, each enrolled cadet completed a baseline assessment. Incident concussions were defined by evidence-based guidelines (Carney et al., 2014). After a diagnosed concussion, research staff recorded the injury's characteristics. These included the injury activity, date and time of injury, injury activity, and the presence of LOC, RGA, PTA, and acute symptoms.

Concussion activities were categorized into sport (varsity, club, intermural level), academy training (physical education, academy training), or free time-related concussions. Free 
time-related concussions were defined as those not occurring during any structured Service Academy activity. The symptom tool used was the Standardized Concussion Assessment Tool (SCAT) symptom scale (McCrory et al., 2009). When possible, cadets completed postconcussion assessments within six hours of injury and 24-48 hours of injury. Cadets also completed SCAT symptoms scales daily until a clinician deemed them asymptomatic or if symptoms persisted for more than two weeks, assessment frequency reduced to weekly. Per institutional protocol, all cadets, regardless of varsity status, completed a RTA protocol before returning to activity.

\subsubsection{Statistical Analysis}

Between 2014 and 2017, 800 incident injuries were collected at the service academies. These 800 injuries occurred among 738 individual cadets, 56 cadets had two concussions, and three cadets had three concussions. The current analyses only evaluated recovery after the first incident concussion.

The days between the injury date and the date when the cadet was determined to be asymptomatic defined the symptom duration period. The number of days between the date the cadet was declared asymptomatic and the date the clinician cleared the cadet for return to activity defined the RTA protocol duration.

Acute symptom burden was defined as the number of symptoms, rather than symptom severity, collected at the six-hour or 24-48 hour time point, based on previous work (Nelson et al., 2016). Using the median split (Nelson et al., 2016), cadets with greater than 11 symptoms post-injury were categorized as having a high symptom burden while those with less than or equal to 11 symptoms were characterized as having a low symptom burden. The presence of 11 or more symptoms is well above the baseline median symptom score (Hanninen et al., 2016).

Similarly, baseline psychological symptoms, measured by the Brief Symptom Inventory18 (BSI-18) (Meachen et al., 2008) were also categorized as high or low. Rather than using a median split, the top quartile for somatization, depression, and anxiety scores were used to categorize high psychological symptoms. The upper quartile, rather than median, was used to define cohorts with clinically meaningful symptom burdens. The median values for each psychological domain were well below clinical cutoff scores (Derogatis, 2001). While lower than clinical cutoff scores, the upper quartile was similar to scores found to indicate clinically meaningful psychological distress (Merport \& Recklitis, 2012). 
Participant characteristics were assessed using Chi-Squared tests for categorical variables. Independent samples t-tests were used for nominal by continuous variables. The significance level was set at $\alpha=0.05$.

Recovery trajectories were assessed across all concussion types and then analyzed by sub-type (sport, academy training, or free time-related). First, univariate tests using KaplanMeier estimates measured the effect of individual demographics, medical history, baseline psychological symptoms, and acute injury characteristics on asymptomatic and RTA protocol durations. The proportional hazard assumption was checked for each test by inspecting the $\log (-$ $\log$ (survival) plots. When the proportional hazard assumption was violated the Wilcoxon test (Wxn) rather than log-rank test (LR) was used (Lee, Desu, \& Gehan, 1975). Tables 3.3.2 and 3.3.4 indicate the test used for each variable. Median duration and 95\% confidence intervals (CI) are reported. Cadets who were missing RTA date but had asymptomatic date $(n=55)$ and cadets missing both RTA and symptomatic dates $(\mathrm{n}=36)$ were censored using the cadet's graduation date or end of study date. Cadets missing the asymptomatic date but had RTA date $(n=91)$ were excluded from the survival analyses.

Results from the univariate tests were used to select potential variables for the multivariate models. A priori, sex was identified as a potential modifying variable and was included as a possible variable in each of the multivariate analyses. Other predictors were included if they had a p-value less than or equal to 0.15. A Cox Proportional Hazard model using stepwise selection generated final models estimating asymptomatic and RTA duration for all concussions, sport, academy training, and free time-related concussions. The Supremum Test tested the proportional hazards assumption. If a variable was found to violate the proportional hazards assumption, the variable was interacted with time (Days until asymptomatic or RTA duration days) and included in the model. Hazard ratios (HR) and 95\% confidence intervals are reported. A mediation analysis was conducted to evaluate the mediating role of symptom resolution on RTA duration (Iacobucci, 2012; Nelson et al., 2016). All statistical analyses were completed using SAS v9.4.

\subsubsection{Results}

\subsubsection{Sample Characteristics}

There were 738 cadets who sustained at least one incident concussion. Cadets with missing data on their sex or sport level were removed from the dataset, leaving $726(n=275$ 
female) individual cadet injuries for analyses. Sample characteristics are presented in Table 3.3.1.

Of these 726 injuries, $42.56 \%(\mathrm{n}=309)$ occurred during sport, 32.37\% $(\mathrm{n}=235)$ during academy training, $15.15 \%(n=110)$ during free time, and $9.92 \%(n=72)$ were missing an injury activity category (Figure 3.3.1). Whether the cadet immediately reported the concussion was available in 698 of 726 concussive injuries, with $45.56 \%(n=318)$ immediately reporting the injury to the medical staff. Males were 1.53 more likely than females to immediately report their concussion (95\% CI: 1.13-2.09), significantly higher than females $(\mathrm{p}=0.007)$. Among those cadets who delayed reporting their injury, the median number of days delayed was 1.00 (IQR: 0.00-3.00) days. There was no significant effect of sex on the number of days until the concussion was reported $(\mathrm{p}>0.05)$.

Table 3.3.1. Sample Characteristics

\begin{tabular}{|c|c|c|}
\hline Variable & $\%(n)$ & Mean (SD) \\
\hline \multicolumn{3}{|l|}{ Sex } \\
\hline Male & $62.12 \%(451)$ & \\
\hline \multicolumn{3}{|l|}{ Race } \\
\hline White & $66.94 \%(486)$ & \\
\hline African American & $8.82 \%(64)$ & \\
\hline Other & $20.67 \%(150)$ & \\
\hline Missing & $3.58 \%(26)$ & \\
\hline \multicolumn{3}{|l|}{ Previous Concussion } \\
\hline Yes & $29.61 \%(215)$ & \\
\hline Missing & $4.96 \%(36)$ & \\
\hline Number of Previous Concussions & & $0.43(0.75)$ \\
\hline \multicolumn{3}{|l|}{ Site } \\
\hline $1=\mathrm{USMA}$ & $38.57 \%(280)$ & \\
\hline $2=$ USAFA & $57.99 \%(421)$ & \\
\hline $3=\mathrm{USCGA}$ & $3.44 \%(25)$ & \\
\hline \multicolumn{3}{|l|}{ Sport Level } \\
\hline Varsity & $36.91 \%(268)$ & \\
\hline Non-Varsity & $59.64 \%(433)$ & \\
\hline Missing & $3.44 \%(25)$ & \\
\hline Age & & $19.14(1.41)$ \\
\hline Height (m) & & $1.75(0.10)$ \\
\hline Weight (kg) & & $74.57(13.92)$ \\
\hline
\end{tabular}


Figure 3.3.1. Frequency of Concussion Types

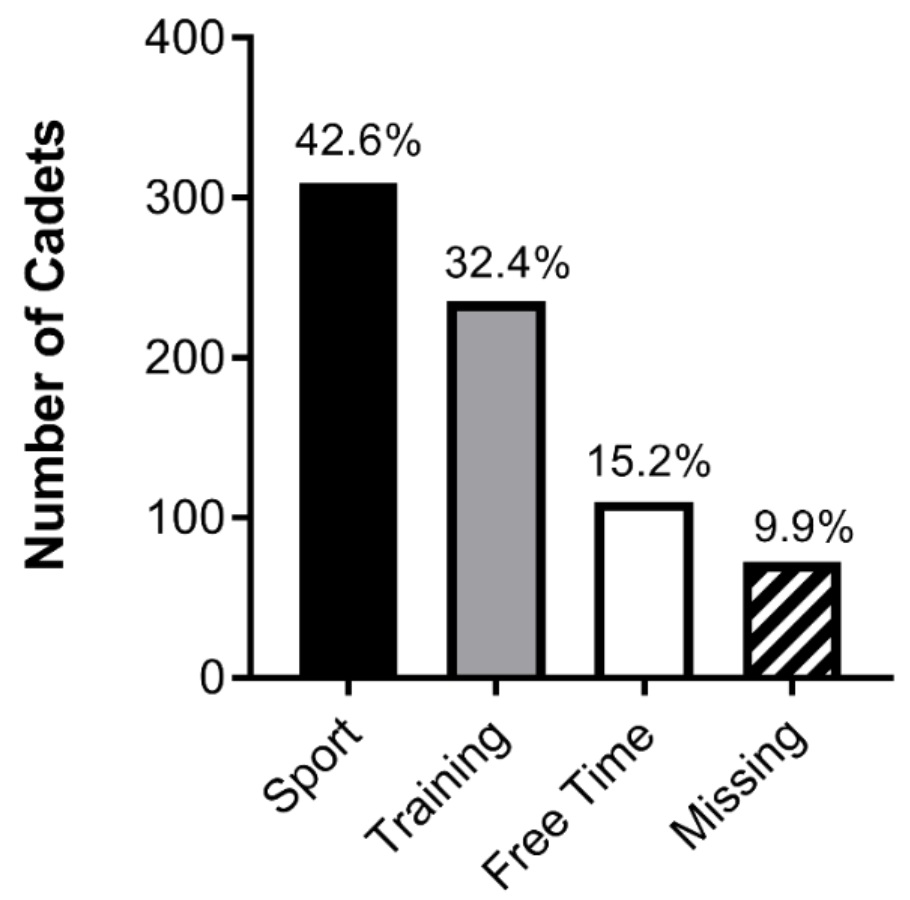

Concussion Type

The median duration until asymptomatic, for any concussion, was 11.00 days (95\% CI: $10.00-12.00)$. By day $7,33.44 \%(n=213)$ became asymptomatic. At day $14,59.18 \%(n=377)$ were asymptomatic and the almost all $(84.14 \%, \mathrm{n}=536)$ were asymptomatic by day 28 . Cadets did not return to full activity/duty until a median of 24.00 days (95\% CI: $23.00-26.00)$. The return to play protocol lasted for 8.00 days (95\% CI: $9.00-9.00)$.

\subsubsection{Predictors of Symptom Duration}

Univariate results revealed a significant effect of concussion type on days until asymptomatic. Injury type was significantly associated with symptom duration $\left(X^{2}=19.08 ; \mathrm{p}<\right.$ 0.0001). Specifically, sport-related concussions had significantly fewer median days until asymptomatic compared to free time-related concussions $(\mathrm{p}<0.0001)$ and tended to have shorter symptom durations than academy-training related concussions $(\mathrm{p}=0.07)$. There was no significant differences academy training-related concussions and free time-related concussions (Table 3.3.2). Among all concussions, male sex, varsity status, immediately reporting the injury, 
no PTA, and low SCAT symptom burden were associated with significantly fewer days until asymptomatic (all p's < 0.05) (Table 3.3.2).

When examining recovery predictors by concussion activity, different associations between predictors and days until asymptomatic emerged. Sport-related concussions had the largest number of associations with recovery time. Varsity sport concussions demonstrated associations between sexes, varsity status, headache, LOC, PTA, immediately reporting the concussions, SCAT symptom number, baseline BSI-18 depression symptoms, and baseline BSI18 anxiety symptoms (Table 3.3.2). The most consistent predictors of symptom recovery across all injury types were SCAT symptoms. 
Table 3.3.2. Kaplan Meier Results for Symptom Duration. Median, $95 \%$ Confidence Interval, Test Statistic

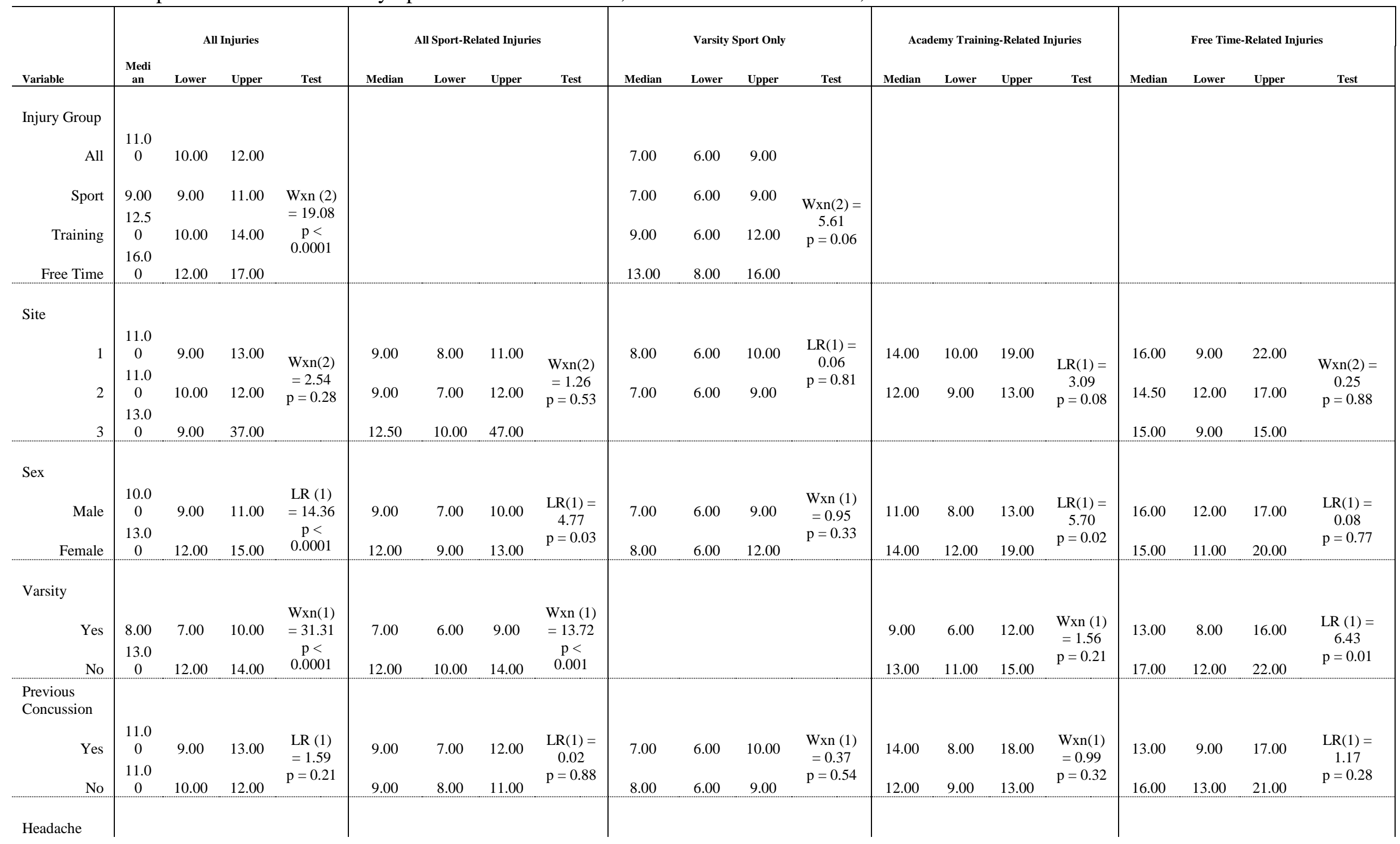




\begin{tabular}{|c|c|c|c|c|c|c|c|c|c|c|c|c|c|c|c|c|c|c|c|c|}
\hline $\begin{array}{l}\text { Yes } \\
\text { No }\end{array}$ & $\begin{array}{c}12.0 \\
0 \\
10.0 \\
0\end{array}$ & $\begin{array}{l}11.00 \\
9.00 \\
\end{array}$ & $\begin{array}{r}13.00 \\
12.00 \\
\end{array}$ & $\begin{array}{c}\text { Wxn (1) } \\
=3.79 \\
\mathrm{p}=0.05\end{array}$ & $\begin{array}{l}11.00 \\
9.00 \\
\end{array}$ & $\begin{array}{l}9.00 \\
8.00 \\
\end{array}$ & $\begin{array}{l}13.00 \\
10.00\end{array}$ & $\begin{array}{c}\mathrm{LR}(1)= \\
4.38 \\
\mathrm{p}=0.03\end{array}$ & $\begin{array}{l}8.00 \\
7.00 \\
\end{array}$ & $\begin{array}{l}5.00 \\
6.00 \\
\end{array}$ & $\begin{array}{l}12.00 \\
9.00 \\
\end{array}$ & $\begin{array}{c}\text { Wxn (1) } \\
=0.49 \\
\mathrm{p}=0.48\end{array}$ & $\begin{array}{l}13.00 \\
12.00\end{array}$ & $\begin{array}{l}10.00 \\
8.00 \\
\end{array}$ & $\begin{array}{l}16.00 \\
14.00 \\
\end{array}$ & $\begin{array}{c}\text { Wxn(1) } \\
=0.77 \\
\mathrm{p}=0.38\end{array}$ & $\begin{array}{l}14.00 \\
16.00 \\
\end{array}$ & $\begin{array}{l}11.00 \\
12.00\end{array}$ & $\begin{array}{l}18.00 \\
21.00 \\
\end{array}$ & $\begin{array}{c}\mathrm{Wxn}(1)= \\
0.01 \\
\mathrm{p}=0.96\end{array}$ \\
\hline $\begin{array}{l}\text { Yes } \\
\text { No }\end{array}$ & $\begin{array}{c}17.0 \\
0 \\
11.0 \\
0\end{array}$ & $\begin{array}{r}8.00 \\
10.00 \\
\end{array}$ & $\begin{array}{l}19.00 \\
12.00 \\
\end{array}$ & $\begin{array}{c}\text { Wxn (1) } \\
=2.34 \\
p=0.13\end{array}$ & $\begin{array}{r}5.00 \\
10.00 \\
\end{array}$ & $\begin{array}{l}2.00 \\
9.00 \\
\end{array}$ & $\begin{array}{l}10.00 \\
12.00\end{array}$ & $\begin{array}{c}\mathrm{Wxn}(1) \\
=5.69 \\
\mathrm{p}=0.02\end{array}$ & $\begin{array}{l}3.50 \\
8.00\end{array}$ & $\begin{array}{l}1.00 \\
7.00 \\
\end{array}$ & $\begin{array}{r}6.00 \\
10.00 \\
\end{array}$ & $\begin{array}{c}\mathrm{LR}(1)= \\
5.11 \\
\mathrm{p}=0.02\end{array}$ & $\begin{array}{l}18.50 \\
12.00\end{array}$ & $\begin{array}{l}6.00 \\
9.00 \\
\end{array}$ & $\begin{array}{l}92.00 \\
13.00 \\
\end{array}$ & $\begin{array}{c}\mathrm{LR}(1)= \\
5.28 \\
\mathrm{p}=0.02\end{array}$ & $\begin{array}{l}18.00 \\
13.50\end{array}$ & $\begin{array}{r}7.00 \\
11.00\end{array}$ & $\begin{array}{l}63.00 \\
16.00\end{array}$ & $\begin{array}{c}\mathrm{Wxn}(1)= \\
3.06 \\
\mathrm{p}=0.08\end{array}$ \\
\hline $\begin{array}{l}\text { Yes } \\
\text { No }\end{array}$ & $\begin{array}{c}12.5 \\
0 \\
11.0 \\
0\end{array}$ & $\begin{array}{l}10.00 \\
10.00\end{array}$ & $\begin{array}{l}17.00 \\
12.00\end{array}$ & $\begin{array}{c}\text { LR (1) } \\
=4.24 \\
\mathrm{p}=0.04\end{array}$ & 10.00 & $\begin{array}{l}4.00 \\
9.00\end{array}$ & $\begin{array}{l}12.00 \\
12.00\end{array}$ & $\begin{array}{c}\mathrm{Wxn}(1) \\
=4.10 \\
\mathrm{p}=0.04\end{array}$ & $\begin{array}{l}6.50 \\
8.00\end{array}$ & $\begin{array}{l}3.00 \\
6.00\end{array}$ & $\begin{array}{l}11.00 \\
9.00\end{array}$ & $\begin{array}{c}\mathrm{LR}(1)= \\
1.43 \\
\mathrm{p}=0.23\end{array}$ & $\begin{array}{l}17.50 \\
12.00\end{array}$ & $\begin{array}{l}8.00 \\
9.00\end{array}$ & $\begin{array}{r}84.00 \\
13.00\end{array}$ & $\begin{array}{c}\mathrm{LR}(1)= \\
7.50 \\
\mathrm{p}= \\
0.006\end{array}$ & $\begin{array}{l}19.00 \\
14.00\end{array}$ & $\begin{array}{l}11.00 \\
11.00\end{array}$ & $\begin{array}{l}54.00 \\
17.00\end{array}$ & $\begin{array}{c}\mathrm{Wxn}(1)= \\
3.83 \\
\mathrm{p}=0.05\end{array}$ \\
\hline $\begin{array}{l}\text { Immediately } \\
\text { Reported } \\
\qquad \text { Yes } \\
\text { No }\end{array}$ & $\begin{array}{c}9.00 \\
13.0 \\
0\end{array}$ & 12.00 & $\begin{array}{l}11.00 \\
14.00 \\
\end{array}$ & $\begin{array}{c}\text { Wxn(1) } \\
=13.04 \\
\mathrm{p} \\
<0.001\end{array}$ & 12.00 & $\begin{array}{r}7.00 \\
10.00 \\
\end{array}$ & $\begin{array}{r}9.00 \\
13.00\end{array}$ & $\begin{array}{c}\text { Wxn (1) } \\
=10.23 \\
\mathrm{p}= \\
0.001\end{array}$ & $\begin{array}{l}7.00 \\
9.00 \\
\end{array}$ & $\begin{array}{l}5.00 \\
7.00 \\
\end{array}$ & $\begin{array}{r}8.00 \\
11.00 \\
\end{array}$ & $\begin{array}{c}\mathrm{Wxn}(1)= \\
1.30 \\
\mathrm{p}=0.25\end{array}$ & $\begin{array}{l}11.00 \\
13.00\end{array}$ & $\begin{array}{r}8.00 \\
10.00 \\
\end{array}$ & $\begin{array}{l}14.00 \\
15.00 \\
\end{array}$ & $\begin{array}{c}\mathrm{LR}(1) \\
=0.84 \\
\mathrm{p}=0.36\end{array}$ & $\begin{array}{l}14.00 \\
16.00 \\
\end{array}$ & $\begin{array}{l}10.00 \\
12.00\end{array}$ & $\begin{array}{l}18.00 \\
20.00\end{array}$ & $\begin{array}{c}\text { Wxn }(1)= \\
0.28 \\
p=0.60\end{array}$ \\
\hline $\begin{array}{l}\text { High SCAT } \\
\text { Number } \\
\qquad \text { High }\end{array}$ & $\begin{array}{c}14.0 \\
0 \\
8.00\end{array}$ & $\begin{array}{r}13.00 \\
7.00\end{array}$ & $\begin{array}{l}16.00 \\
9.00\end{array}$ & $\begin{array}{c}\text { LR }(1) \\
=52.30 \\
\mathrm{p}< \\
0.0001\end{array}$ & $\begin{array}{r}13.00 \\
7.00 \\
\end{array}$ & $\begin{array}{l}11.00 \\
6.00\end{array}$ & $\begin{array}{l}15.00 \\
9.00\end{array}$ & $\begin{array}{c}\mathrm{LR}(1)= \\
26.25 \\
\mathrm{p}< \\
0.0001\end{array}$ & $\begin{array}{l}11.50 \\
6.00\end{array}$ & $\begin{array}{l}8.00 \\
5.00\end{array}$ & $\begin{array}{r}13.00 \\
7.00\end{array}$ & $\begin{array}{c}\mathrm{W} \times \mathrm{n}(1)= \\
17.47 \\
\mathrm{p}< \\
0.001\end{array}$ & $\begin{array}{l}15.00 \\
9.00\end{array}$ & $\begin{array}{r}13.00 \\
6.00\end{array}$ & $\begin{array}{l}18.00 \\
12.00\end{array}$ & $\begin{array}{c}\mathrm{LR}(1)= \\
17.42 \\
\mathrm{p}< \\
0.0001\end{array}$ & $\begin{array}{l}17.00 \\
12.00\end{array}$ & $\begin{array}{l}14.00 \\
10.00\end{array}$ & $\begin{array}{l}22.00 \\
16.00\end{array}$ & $\begin{array}{c}\mathrm{LR}(1)= \\
2.77 \\
\mathrm{p}=0.10\end{array}$ \\
\hline $\begin{array}{l}\text { High BSI } \\
\text { Somatization } \\
\text { High } \\
\text { Low }\end{array}$ & $\begin{array}{c}11.0 \\
0 \\
11.0 \\
0\end{array}$ & 10.00 & $\begin{array}{r}14.00 \\
12.00\end{array}$ & $\begin{array}{c}\mathrm{LR}(1)= \\
0.70 \\
\mathrm{p}=0.40\end{array}$ & $\begin{array}{l}13.00 \\
9.00\end{array}$ & $\begin{array}{l}9.00 \\
8.00\end{array}$ & $\begin{array}{l}18.00 \\
11.00 \\
\end{array}$ & $\begin{array}{l}\text { LR }(1) \\
=3.40 \\
\mathrm{p}=0.07\end{array}$ & $\begin{array}{l}10.00 \\
7.00\end{array}$ & $\begin{array}{r}7.00 \\
6.00 \\
\end{array}$ & $\begin{array}{l}14.00 \\
8.00\end{array}$ & $\begin{array}{c}\mathrm{W} \times \mathrm{nn}(1)= \\
2.67 \\
\mathrm{p}=0.10\end{array}$ & $\begin{array}{r}7.00 \\
13.00 \\
\end{array}$ & $\begin{array}{r}6.00 \\
12.00\end{array}$ & $\begin{array}{r}12.00 \\
15.00\end{array}$ & $\begin{array}{c}\mathrm{Wxn}(1) \\
=5.31 \\
\mathrm{p}=0.02\end{array}$ & $\begin{array}{l}14.00 \\
16.00 \\
\end{array}$ & $\begin{array}{r}7.00 \\
12.00 \\
\end{array}$ & $\begin{array}{l}16.00 \\
20.00 \\
\end{array}$ & $\begin{array}{c}\mathrm{Wxn}(1)= \\
1.81 \\
\mathrm{p}=0.18\end{array}$ \\
\hline $\begin{array}{l}\text { High BSI } \\
\text { Depression } \\
\text { High } \\
\text { Low }\end{array}$ & $\begin{array}{c}12.0 \\
0 \\
11.0 \\
0\end{array}$ & 10.00 & $\begin{array}{r}13.00 \\
12.00\end{array}$ & $\begin{array}{c}\mathrm{LR}(1)= \\
0.82 \\
\mathrm{p}=0.36\end{array}$ & $\begin{array}{l}13.00 \\
9.00\end{array}$ & $\begin{array}{l}9.00 \\
8.00\end{array}$ & $\begin{array}{l}17.00 \\
10.00 \\
\end{array}$ & $\begin{array}{c}\mathrm{LR}(1)= \\
4.12 \\
\mathrm{p}=0.04\end{array}$ & $\begin{array}{l}13.00 \\
7.00 \\
\end{array}$ & $\begin{array}{l}9.00 \\
6.00 \\
\end{array}$ & $\begin{array}{l}15.00 \\
8.00\end{array}$ & $\begin{array}{c}\mathrm{Wxn}(1)= \\
7.79 \\
\mathrm{p}=0.005\end{array}$ & $\begin{array}{r}8.00 \\
13.50 \\
\end{array}$ & $\begin{array}{r}6.00 \\
12.00\end{array}$ & $\begin{array}{l}12.00 \\
16.00\end{array}$ & $\begin{array}{c}\text { Wxn (1) } \\
=5.18 \\
\mathrm{p}=0.02\end{array}$ & $\begin{array}{r}21.00 \\
15.00 \\
\end{array}$ & $\begin{array}{r}9.00 \\
12.00 \\
\end{array}$ & $\begin{array}{l}48.00 \\
17.00 \\
\end{array}$ & $\begin{array}{c}\mathrm{LR}(1)= \\
0.16 \\
\mathrm{p}=0.69\end{array}$ \\
\hline
\end{tabular}


Across all concussions, Cox Proportional Hazard models revealed that varsity status and SCAT symptom number were associated with days until asymptomatic (all p's $<0.05$ ) (Figure 3.3.2). Varsity status and SCAT number were also found to be time-dependent covariates (all p's <0.05). Varsity cadets (HR: 1.76 95\% CI: 1.38-2.23) (Figure 3.3.2a) had faster recovery rates, but these effects decreased over time. High SCAT number at baseline (HR: $0.4195 \%$ CI: 0.320.53) (Figure 3.3.2b) was associated with slower recovery with the effect of SCAT number decreasing with time.

Figure 3.3.2. Kaplan Meier Plots of Days until Asymptomatic among All Concussions. Survival curve with $95 \%$ Confidence Interval

A. Varsity Status

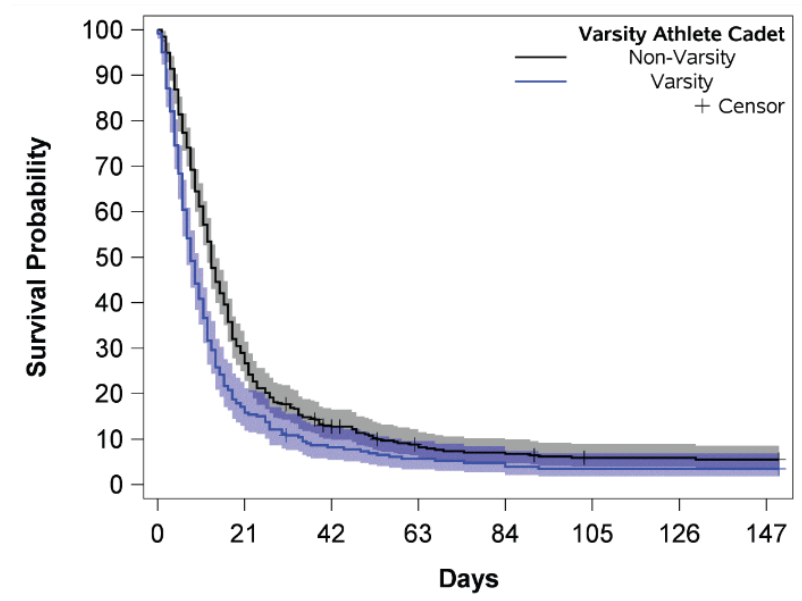

B. SCAT Symptom Number Group

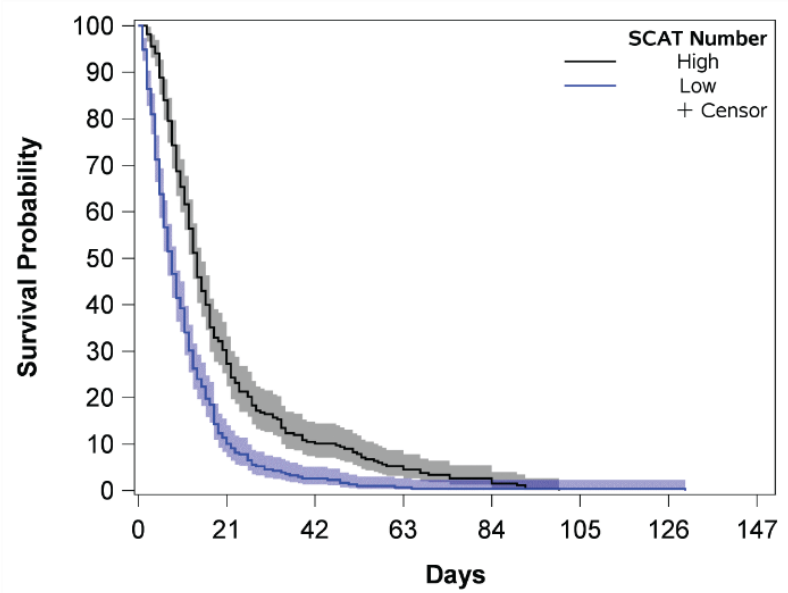

Within sport-related injuries, varsity status and SCAT number were associated with time until asymptomatic (both p's $<0.05$ ). Both varsity status and SCAT number were also time dependent covariates. Varsity cadets had a 9 fold increase rate of becoming asymptomatic than non-varsity cadets (HR: 9.19 95\% CI: 5.40-15.64). However, this effect decreased over time, with the hazard rate decreasing from 4.21 to 1.93 between 7 and 14 days post-concussion. While the average effect of high SCAT number was associated with 1.59 times increased symptom recovery rate (95\% CI: 1.05-2.39). Faster recovery was only observed within the first seven days. After day 7 post-concussion, high SCAT symptom number was associated with slower recovery $(\mathrm{HR}<0.82)$. 
Table 3.3.3. Hazard Ratio and $95 \%$ Confidence Interval - Multivariate Models of Days until Asymptomatic

\begin{tabular}{lcccc}
\hline & All Concussions & Any Sport & $\begin{array}{c}\text { Varsity Sport } \\
\text { Only }\end{array}$ & $\begin{array}{c}\text { Academy } \\
\text { Training-Related }\end{array}$ \\
$\begin{array}{lccc}\text { Free Time- } \\
\text { Related }\end{array}$ \\
\hline Varsity & $1.76(1.38-2.23)$ & $9.19(5.40-15.64)$ & & $1.91(1.13-3.23)$ \\
High SCAT Number & $0.41(0.32-0.53)$ & $1.59(1.05-2.39)$ & $0.49(0.34-0.70)$ & $0.57(0.43-0.76)$ \\
High BSI-18 Anxiety & & & & $1.43(1.06-1.94)$ \\
Varsity*Days Asymptomatic & $0.99(0.97-1.00 \dagger)$ & $0.90(0.87-0.93)$ & & \\
SCAT*Days Asymptomatic & $1.02(1.01-1.04)$ & $0.95(0.94-0.97)$ & & \\
\hline N & & & \\
\hline
\end{tabular}

Note: Multivariate results, each hazard ratio controlling for each other variable listed in the model

$\dagger$ Value less than 1.00 
Within Varsity sport-related injuries only SCAT number was significantly associated with symptom duration ( $\mathrm{p}<0.0001$ ). Increased acute SCAT number was associated with a 51\% slower symptom recovery than varsity cadets with low SCAT symptom number (Table 3.3.3). Within academy training-related concussions, SCAT number and baseline BSI-18 anxiety scores were associated with symptom duration (Table 3.3.3). Again, higher acute SCAT symptom number was associated with significantly longer symptom duration (Table 3.3.3). High baseline anxiety symptoms were associated with a $43 \%$ faster recovery than lower baseline anxiety symptoms. Unlike all other concussion sub-types, there was no significant effect of acute SCAT symptom number on symptom duration within free time-related concussions. Instead, varsity was associated with significantly shorter symptom duration (Table 3.3.3).

\subsubsection{Predictors of Return to Play Protocol Duration}

Site was found to influence RTA duration within all injuries, sport, varsity sport, and academy training-related injuries (All p's < 0.05) (Table 3.3.4). Male sex was associated with fewer RTA protocol days within all injuries, academy training, and free-time related concussions (all p's < 0.05). Similarly, varsity athlete cadets spent fewer days within an RTA protocol than non-varsity cadets within all concussions and academy training-related concussions (all p's $<0.05)$. Varsity status was associated with significantly shorter RTA protocol duration within all concussions and sport-related concussions. Finally, high SCAT number was significantly associated with longer RTA protocol days for all concussions, sport, and varsity sport-related concussions (all p's < 0.05) (Table 3.3.4). 
Table 3.3.4. Kaplan Meier Results for RTA Protocol Duration. Median, 95\% Confidence Interval, Test Statistic

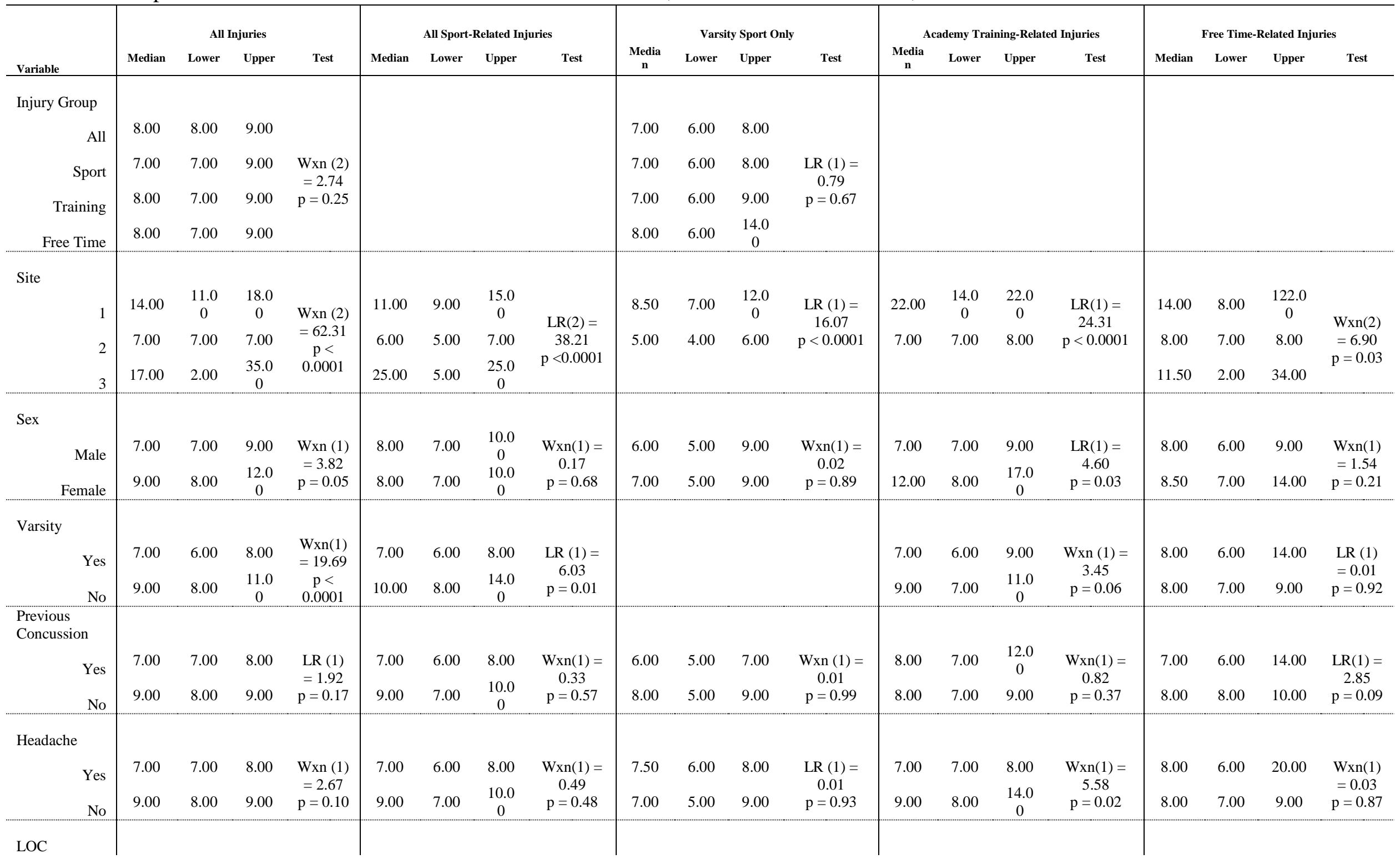




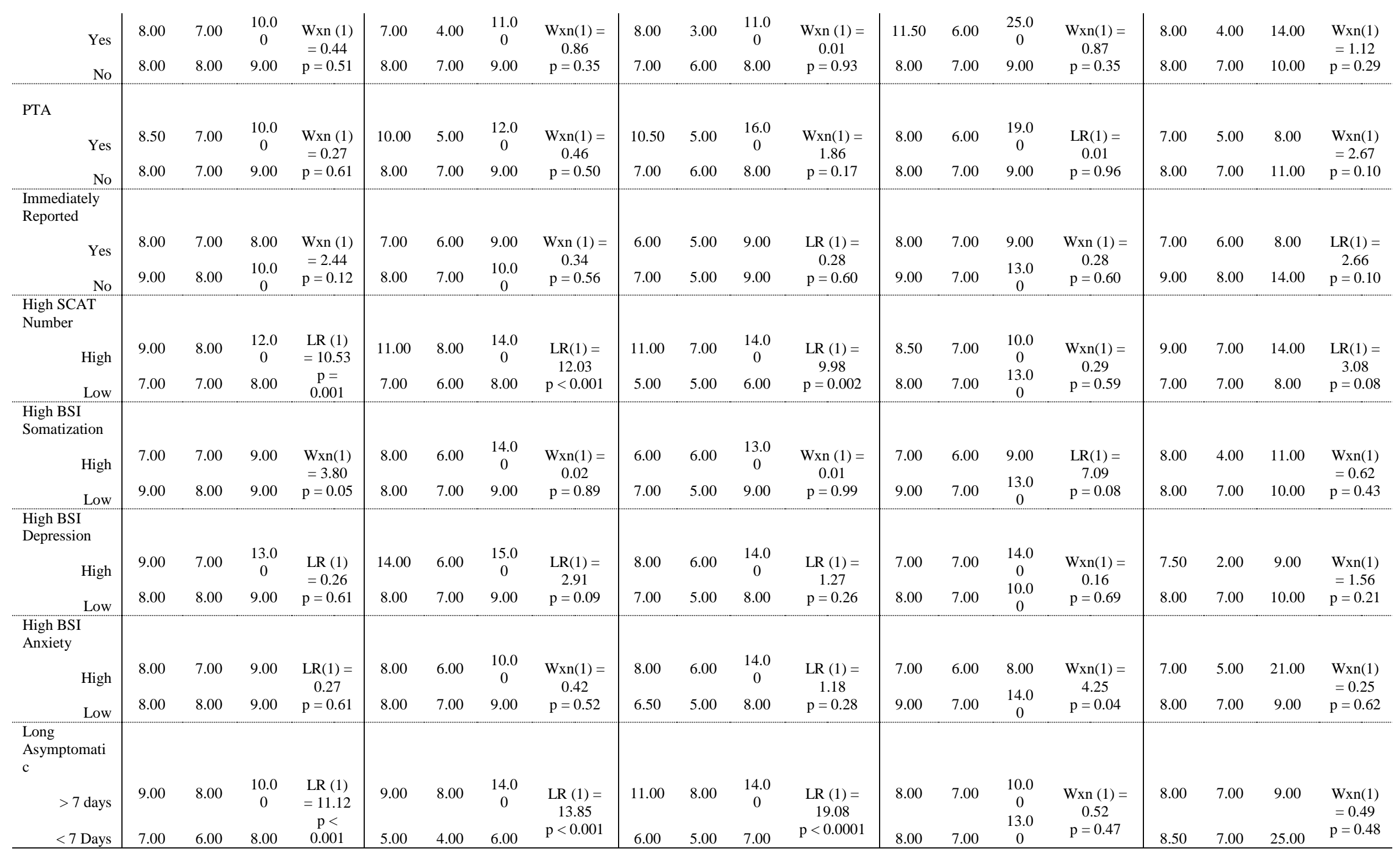


Multivariate Cox Proportional Hazard models found site was significantly associated with RTA protocol duration among each concussion type. Among all concussions, RTA protocol duration was influenced by varsity status and SCAT number. Varsity status was associated with $76 \%$ faster RTA protocol duration than non-varsity cadets (Table 3.3.5). However, this effect decreased with time. High SCAT number immediately after a concussion was associated with a 27\% slower RTA duration (Table 3.3.5).

Within sport-related concussions, varsity status was associated with significantly shorter RTA duration while high SCAT number was associated with longer RTA protocol duration (Table 3.3.5). High SCAT number post-concussion was also associated with a 33\% shorter RTA protocol among varsity sport-related concussions (Table 3.3.5). Additionally, symptom durations of more than 7 days were associated with significantly longer RTA protocol durations $(\mathrm{p}=0.01)$.

Concussions sustained during academy training had RTA protocols significantly influenced by sex and headaches at baseline. Male sex and headaches at baseline significantly reduced the RTA protocol for academy training (Table 3.3.5). No significant predictors of RTA protocol duration were determined among free time-related concussions beyond site. 
Table 3.3.5. Hazard Ratio and 95\% Confidence Interval - Multivariate Models of RTA Protocol Duration

\begin{tabular}{|c|c|c|c|c|c|}
\hline & All Concussions ${ }^{¥}$ & All Sport ${ }^{¥}$ & $\begin{array}{c}\text { Varsity Sport } \\
\text { Only }\end{array}$ & $\begin{array}{c}\text { Academy } \\
\text { Training-Related }\end{array}$ & $\begin{array}{c}\text { Free Time- } \\
\text { Related }^{\ddagger}\end{array}$ \\
\hline Male & & & & $1.44(1.06-1.95)$ & \\
\hline Varsity & $1.76(1.36-2.29)$ & $1.44(1.11-1.88)$ & & & \\
\hline High SCAT Number & $0.77(0.65-0.91)$ & $0.60(0.46-0.79)$ & $0.67\left(0.45-1.00^{\dagger}\right)$ & & \\
\hline $\begin{array}{l}\text { Headaches past } 3 \\
\text { months }\end{array}$ & & & & $1.39(1.03-1.87)$ & \\
\hline $\begin{array}{l}\text { >7 Days Until } \\
\text { Asymptomatic }\end{array}$ & & & $0.62(0.42-0.91)$ & & \\
\hline Varsity*RTA Duration & $0.97(0.96-0.99)$ & & & & \\
\hline
\end{tabular}




\subsubsection{Mediation of Acute Injury Symptoms by Symptom Resolution Duration}

Duration of symptomatic and RTA protocol periods were significantly correlated $(\mathrm{r}=$ $0.16 ; \mathrm{p}=0.0001$ ). Additionally, a symptom resolution time greater than seven days was associated with significantly longer RTA durations for varsity sport-related concussions (Table 3.3.5). Due to the possible relationship and influence of symptom duration on RTA protocol, a Cox Proportional Hazard model of RTA duration, controlling for symptom duration was evaluated. Multivariate models for sport and varsity sport-related concussions found that a symptom duration greater than seven days was significantly associated with RTA duration, controlling for other covariates (Table 3.3.5).

Within varsity sport-related concussions, the duration until asymptomatic was hypothesized to mediate the relationship between SCAT symptoms and RTA protocol duration (Figure 3.3.4a). High SCAT symptom number (OR: 3.74 95\% CI: 2.61-5.44) significantly estimated whether a cadet required more than seven days to become asymptomatic. Additionally, symptom duration significantly estimated RTA protocol duration $(p=0.0141)$ controlling for site and SCAT symptoms. The mediation model found a significant mediation effect for symptom duration and high SCAT symptom number $(\mathrm{Z}=7.52 ; \mathrm{p}<0.0001)$. Thus, symptom resolution duration did not completely mediate the relationship between SCAT symptom number and RTA protocol duration. SCAT symptom number was still significant estimators of RTA protocol duration ( $\mathrm{p}<0.05$; Table 3.3.5).

Figure 3.3.4. Mediation Models of RTA Protocol Duration

A.

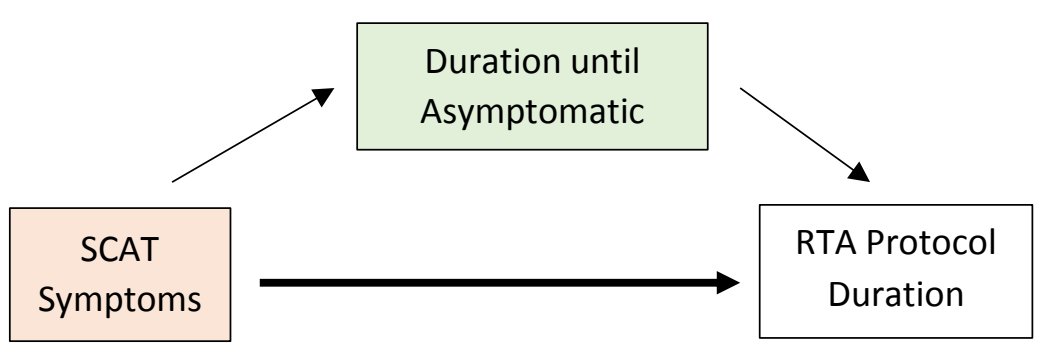




\subsubsection{Discussion}

The most significant findings of this investigation were that individual and acute injury characteristics contribute to symptom duration and RTA protocol duration; including the presence of 11 or more symptoms within 48 hours of a concussion is the most robust and consistent estimator of longer symptom and RTA protocol durations. This finding provides support for hypothesized individual and injury-related factors associated with symptom duration (Iverson et al., 2017). Moreover, by separating the recovery period into symptomatic and RTA protocol durations, we identified factors relevant to recovery versus clinical management. As previous studies have examined total time loss (Asplund et al., 2004; Covassin et al., 2016; Davis-Hayes et al., 2017; Pfaller et al., 2016), it is important to note there are distinct but related processes contributing to concussion recovery. While guidelines have suggested that clinicians manage individuals differently given their medical history, age, and future injury risk (Doolan et al., 2012), no study to our knowledge has systematically evaluated what factors influence clinical management.

\subsubsection{Symptom Duration}

Across all injury types, different individual and acute injury characteristics estimated symptom and RTA protocol duration. Pre-injury characteristics including sex, somatization, and previous concussion are posited to predict recovery (Iverson et al., 2017). Males were hypothesized to have shorter recovery durations after a concussion. However, this hypothesis was not supported. There was no significant effect of sex when assessed using multivariate models in the current analysis. Contrary to previous athletes studies (Henry, Elbin, Collins, Marchetti, \& Kontos, 2016; Miller et al., 2016) (Heyer et al., 2016; Zuckerman et al., 2014) (Davis-Hayes et al., 2017), male cadets did not have shorter symptom resolution after a sportrelated concussion. In the studies finding differences across sexes, the samples contained wider age ranges (Henry et al., 2016; Zuckerman et al., 2014). In studies with samples similar to the current study, no sex effect has been observed (Nelson et al., 2016; Wasserman et al., 2016). Moreover, similar to the current study, those investigations that used multivariate models to adjust for multiple covariates found no sex effect (McCrea et al., 2013; Nelson et al., 2016). Thus, similar to previous multivariate assessments of recovery, sex was not significantly associated with symptom duration. Overall, across all concussions, there is little evidence for a sex effect on symptom duration when accounting for other variables. 
Somatization is thought to mediate the relationship between acute symptom burden and recovery (Nelson et al., 2016). Moreover, this effect is thought to be unique and unrelated to other psychological symptoms including depression and anxiety (Nelson et al., 2016). The current study found no multivariate effect of somatization. Instead, baseline anxiety symptom scores were associated with longer symptom durations for academy training-related concussions. The cadet population is a unique healthy sample, devoid of many medical pre and comorbidities. Thus it is also possible that they experience fewer somatization symptoms. Moreover, cadets experience high stress due to increased physical demands of the Academies (Lee et al., 1992) and reduced sleep (Miller et al., 2010). Cadets may experience fewer fluctuations in somatization but more significant changes in anxiety levels. Thus, unlike the findings by Nelson and colleagues (Nelson et al., 2016), the current study indicates that anxiety symptoms, rather than somatization symptoms were associated with symptom recovery.

Previous concussions have also been hypothesized to increase recovery time (Iverson et al., 2017). If a second concussion occurs when the brain is still recovering from the first injury, a more pronounced and prolonged injury response is produced by the second concussion (Prins, Alexander, et al., 2013; Vagnozzi et al., 2008). Moreover, Eisenberg et al. 2013 (Eisenberg et al., 2013) investigated the effect time between concussions has on symptom duration. Individuals with more than one concussion or a concussion within the previous year were more likely to have longer symptom durations (Eisenberg et al., 2013). While time from a previous concussion was not evaluated in the current study, the effect of multiple concussions was. Contrary to the hypothesis, having a previous concussion was not significantly associated with symptom durations for any concussion type. While animal models suggests a second concussion within a short period of time extends recovery time (Prins, Alexander, et al., 2013), the current study found no effect of a previous recovery on symptom duration. Thus, in the current study, previous concussive injuries could have occurred outside the hypothesized period of vulnerability, or other psychological factors may also contribute. A cadet with a previous concussion may have increased concussion knowledge speeding their recovery, or they may exhibit negative concussion disclosure behavior. In a study of high school athletes, those with increased previous concussions were $125 \%$ more likely to participate in practice and $67 \%$ more likely to participate in games while experiencing symptoms (Register-Mihalik, Valovich McLeod, Linnan, Guskiewicz, \& Marshall, 2017). Cadets may also be concealing concussion symptoms, 
arbitrarily speeding their symptom recovery. However, more research examining duration from previous concussion and cadet reporting behaviors is needed to understand the role of previous concussion on symptom recovery.

The current study's findings are consistent with recent findings that the acute symptom burden predicts symptom duration (McCrea et al., 2013; Meehan, Mannix, Monuteaux, Stein, \& Bachur, 2014; Nelson et al., 2016; Resch et al., 2015). Specifically, increasing symptoms within 48 hours of concussion significantly extends symptom recovery. Notably, these previous studies evaluated recovery from sport-related concussions. The current study adds to the evidence for acute symptom burden influencing recovery after a sport concussion but also expands the existing evidence to non-sport concussions.

\subsubsection{Return to Activity Protocol}

Finally, RTA protocol duration was associated with a different pattern of factors than symptom duration. To our knowledge, no study to date has investigated factors related to RTA duration. A few studies have investigated total time lost due to concussion (Asplund et al., 2004; Covassin et al., 2016; Davis-Hayes et al., 2017; Pfaller et al., 2016), which includes both symptom and RTA duration periods.

Multivariate analyses revealed a significant effect of sex on RTA protocol duration. The effect of sex was only observed in academy-related concussions. Males had significantly shorter RTA durations than females. This finding may indicate that non-sport injuries among females are being managed more conservatively, whereas there is more uniform management within athletic and sports-medicine departments. This difference also could be due to females experiencing a greater symptom re-occurrence when completing the RTA protocol. However, in studies investigating the likelihood of concussion symptoms re-occurring after exercise, there was no difference between males and females (O'Brien, Howell, Pepin, \& Meehan, 2017). Thus, females are not more likely to re-experience symptoms when beginning exercise during the RTA protocol, and it is more likely they are managed more conservatively.

Cadets who had greater than 11 SCAT symptoms within 48 hours of their injury were more likely to have longer RTA durations. The effect of SCAT symptom burden was observed for all concussions, sport, and varsity sport concussions. Moreover, asymptomatic duration was found to mediate a portion of the relationship between high SCAT burden and RTA duration. Thus, cadets with greater initial SCAT scores have longer symptom duration, which influences 
RTA duration. The role of SCAT symptoms and RTA protocol duration is a novel finding, not only do SCAT symptoms influence subsequent symptom duration, but there is a direct and indirect effect on RTA protocol duration. The reason for longer RTA protocol duration is unclear. It is possible that clinicians managed cadets with higher acute symptom burdens more conservatively. Alternatively, a higher symptom burden post-concussion is associated with increased likelihood of symptoms re-emerging during exercise within the RTA protocol. If symptoms re-emerge during the RTA protocol, the duration would extend as cadets must be symptom-free for 24 hours before progressing to the next stage (McCrory et al., 2017). Further investigation is needed to elucidate whether changes in RTA duration are due to differences in management, the frequency of medical visits, or re-emerging symptoms.

\subsubsection{Limitations}

The current study is not without limitation. First, while the study prospectively collected concussive injuries across sport and non-sport domains, it is possible not all concussions were collected. Under-reporting (LaRoche et al., 2016; McCrea et al., 2004) or delayed reporting may cause some injuries to be missed. Additionally, there was missing data for symptom and RTA protocol durations. These missing data were assumed to be missing at random, but not completely at random. There was no clear pattern between missing data and the recovery time predictors. Finally, the current study investigated the first incident concussion sustained. It is possible that multiple concussions induce different recovery patterns.

\subsubsection{Conclusion}

This study identifies the natural history of incident concussion and multiple concussion recovery sub-types. Moreover, specific factors are associated with symptom versus RTA protocol durations. Acute symptom burden is the most consistent and robust predictor of symptom versus and protocol durations. However, the particular symptoms driving symptom and protocol durations are unknown. Further research is needed to determine the particular mechanisms for increased RTA protocol durations. In particular, more research is needed to parse out physiologically driven recovery factors relative to environmental or clinical management factors. 


\subsection{Project 3: Symptom Predictors of Normal and Abnormal Recovery}

\subsubsection{Introduction}

Concussion, or mild traumatic brain injury, affects 42 million individuals annually worldwide (Cassidy et al., 2004). Most individuals return to pre-injury levels of clinical performance within 7-10 days (McCrea et al., 2013; Nelson et al., 2013; Teel et al., 2017). Thus, the recent International Concussion Consensus Meeting defines normal recovery among adults as occurring within 14 days (McCrory et al., 2017). However, approximately 5-6\% of concussed individuals take longer than 28 days to recover (Nelson et al., 2016; Wasserman et al., 2016). Previous studies have attempted to find individual and injury characteristics associated with those failing to recover by day 28 (McCrea et al., 2013). In particular, total acute symptom burden has been the most consistent injury characteristic associated with recovery duration (Iverson et al., 2017). However, it is unknown which specific symptoms influence recovery. Identifying individual symptoms lays the groundwork for potential interventions.

Previous efforts to define the natural history of concussion have coalesced recovery as either the point that symptoms resolve (McCrea et al., 2013) or when the individual is cleared to return to activity(Lau et al., 2009; Lau et al., 2011). Return to activity typically occurs five to seven days after symptom resolution (Doolan et al., 2012). The duration until asymptomatic versus return to activity measures different constructs and conflates clinical interpretation. Symptom duration is likely influenced by physiological recovery, produced by the underlying perturbed brain physiology caused by concussion (Giza \& Hovda, 2014). Return to activity decisions are clinician-dependent and thus might be influenced by differences in management (Doolan et al., 2012) in addition to underlying physiological recovery. The recovery issue becomes further confused as the definition of prolonged recovery was not consistent in the previous studies, ranging from 7-21 days (Iverson, 2007; Lau et al., 2009; Lau et al., 2011; McCrea et al., 2013). Most recently a study of concussed young adults $(\mathrm{n}=536)$ identified $89 \%$ achieve symptom resolution within 28 days (Chapter 3.3) (O'Connor, In Prep). The variable time frames and recovery definitions make it difficult to identify consistent factors associated with prolonged recovery.

The number and severity of acute concussion symptoms have the strongest evidence for estimating recovery duration (Iverson et al., 2017). Previous studies have grouped symptoms into clusters to examine their association with recovery duration (Franke, Czarnota, Ketchum, \& 
Walker, 2015; Lau et al., 2011; Piland, Motl, Ferrara, \& Peterson, 2003). However, using symptom clusters prohibits identifying individual symptoms associated with recovery. Moreover, without validation and different symptom scales, specific clusters and symptoms within each cluster vary across studies (Franke et al., 2015; Lau et al., 2011; Piland et al., 2003). Identifying individual symptoms associated with recovery has the potential to be more clinically useful and may identify intervention targets.

Some work has attempted to identify individual symptoms associated with recovery (Erlanger et al., 2003; Lau et al., 2009; Teel et al., 2017). In a study of high school football players, concussed athletes were classified as having a prolonged recovery if they did not recover within ten days(Lau et al., 2009). The prolonged recovery group had increased migraine, sleep, and cognitive-related symptoms when compared to the normal recovery group (Lau et al., 2009). A follow-up study attempted to identify individual on-field signs/symptoms as predictors of abnormal recovery (Lau et al., 2011). Results revealed that only dizziness predicted recovery and that dizziness was associated with a six-fold increased odds of recovery more than 21 days versus less than seven days (Lau et al., 2011). Both studies were limited to high school football players, leaving many questions around females, collegiate varsity athletes, and non-sport related concussions. In a study of male and female athletes, only self-reported memory problems were associated with longer recovery(Erlanger et al., 2003). However, each of these studies did not account for baseline symptoms (Erlanger et al., 2003; Lau et al., 2009; Lau et al., 2011). Since concussion symptoms are reported by non-concussed individuals (Asken, Snyder, et al., 2017), specifying concussion symptoms relative to naturally occurring symptoms would likely improve concussion symptom specificity in predicting recovery. Finally, previous studies used total time loss after injury (i.e. time to asymptomatic plus return to activity) as a measure of recovery (Erlanger et al., 2003; Lau et al., 2009; Lau et al., 2011). Consequently, these studies are unable to parse how symptoms relate to recovery (symptom resolution) versus clinical management (RTP protocol duration).

Thus, the primary objective of the current study is to identify the specific post-concussive symptoms associated with time to asymptomatic. Since concussion symptoms are nonspecific(Chin et al., 2016), we hypothesize that symptoms not commonly reported at baseline (Covassin et al., 2006; Piland, Ferrara, Macciocchi, Broglio, \& Gould, 2010; Shehata et al., 2009) will be the most informative symptoms post-concussion. Specifically, we hypothesize that 
dizziness (Lau et al., 2011), nausea or vomiting, blurred vision, balance problems, sensitivity to light or sound, difficulty remembering will be most sensitive and specific to recovery duration (Asken, Snyder, et al., 2017; Wasserman et al., 2016). Finally, since symptoms are known to evolve post-concussion (Marshall et al., 2015), we expect that different symptom profiles captured immediately following injury versus subsequently will differentially estimate recovery.

\subsubsection{Methods}

\subsubsection{Study Design}

The current study is part of a larger joint effort by the U.S. Department of Defense (DoD) and National Collegiate Athletic Association (NCAA). This partnership funded the Concussion, Assessment, Research, and Education (CARE) Consortium, a multi-site investigation on the natural history of concussion. A detailed description of the CARE organization has been published previously (Broglio, McCrea, et al., 2017). The protocol was reviewed and approved by site-level Institutional Review Boards (IRB) and the DoD Human Research Protections Office (HRPO) (Broglio, McCrea, et al., 2017). Participants provided written consent before data collection.

Between 2014 and 2017 all cadets at the Military Service Academies were eligible to enroll in CARE. Before each academic year, each enrolled cadet completed a baseline assessment. Incident concussions were defined by evidence-based guidelines as a change in brain function following a force to the head, which may or may not be accompanied by temporary loss of consciousness (Carney et al., 2014).

The Standardized Concussion Assessment Tool (SCAT) symptom scale (McCrory, Meeuwisse, Aubry, Cantu, Dvořák, Echemendia, Engebretsen, Johnston, Kutcher, Raftery, Sills, et al., 2013) was implemented when possible with concussed cadets within six hours of injury and 24-48 hours of injury. Concussed cadets then completed SCAT symptoms scales daily until a clinician deemed them ready to begin the return to play progression (i.e. asymptomatic) or if symptoms persisted for more than two weeks, assessment frequency reduced to weekly. The SCAT symptom scale consists of 22 symptoms, each rated on a 6 point Likert scale. Symptoms were treated as continuous. Post-concussion symptom scores were evaluated using the $\leq 6$ hour and 24-48 hour post-injury time points. Additionally, difference scores were calculated by subtracting the baseline symptom scores from the $\leq 6$ hour and 24-28 hour symptom scores. 
Consequently, four models were evaluated: symptom scores within 6 hours, symptom difference scores at 6 hours, symptom scores at 24-48 hours, and symptom difference scores at 25-48 hours.

\subsubsection{Statistical Methods}

Between 2014 and 2017, 800 incident injuries were collected at the service academies. These 800 injuries occurred among 738 individual cadets, 56 cadets had two concussions, and three cadets had three concussions. For the current analyses only recovery after the first incident concussion was evaluated. Of the 800 concussions, 62 were removed for being a repeat concussion, 124 were removed for missing the date declared asymptomatic, and 12 were removed for missing sex or injury activity data. Thus, 602 concussions were included in the current analyses.

Time until asymptomatic was defined as the days between the injury date and the date when the cadet was determined to be asymptomatic. Kaplan Meier estimates of survival were used to establish recovery trends. At 14 days post-concussion 59\% ( $\mathrm{n}=377)$ of cadets were asymptomatic and $84 \%(\mathrm{n}=536)$ were asymptomatic by 28 days which is lower, but consistent with previous studies finding 95\% symptom resolution by 28 days (Nelson et al., 2016; Wasserman et al., 2016). The normal and abnormal recovery cutoff was a clinical indicator and while the majority (59\%) of cadets were asymptomatic by day 14, a clinically substantial proportion (41\%) were still symptomatic. Consequently, 28 days was used as the threshold of abnormal recovery, rather than 14 days as suggested for adults by the recent Concussion Consensus Statement (McCrory et al., 2017).

Classification and Regression Tree analysis (CART) was implemented to predict normal and abnormal recovery using the symptoms reports noted above. CART is a machine learning predictive modeling method (Breiman, Friedman, Stone, \& Olshen, 1984). The predictive algorithm builds a decision tree where predictor variables are dichotomized and used to classify the probability of a certain outcome. The resulting decision tree aims to classify the sample population with maximal sensitivity and specificity. Unlike logistic regression, CART generates a powerful but interpretable explanatory model. While logistic regression and CART are both predictive models, logistic regression can only classify an outcomes likelihood on a single linear decision boundary. Unlike logistic regression, CART can create multiple decision boundaries, providing greater flexibility. Additionally, CART automatically considers all possible variable interactions, whereas in logistic regression each interaction would need to be explicitly specified. 
Since the objective of the current study is to provide clinicians with clear indicators of normal and abnormal recovery, CART is a superior tool, providing information on clinically and statistically relevant recovery predictors. Additionally, CART method is non-parametric and thus robust for non-normally distributed data, such as SCAT symptom scores (Breiman et al., 1984; Morgan, 2014).

Training and testing datasets were derived from the 602 sample. First, the database was shuffled to eliminate any patterns within the database. Next, $80 \%(n=402)$ observations were selected for the training database while the remaining $20 \%$ of observations were used to generate the testing database. A CART model was first trained on the training dataset then validated using the testing dataset to assess accuracy, generalizability, and evaluate over-fitting of the model. Analyses were completed in RStudio 3.3.3 using the "caret" package. Repeated cross-validation was used with 50-fold cross-validation repeated three times. Receiver Operating Characteristics (ROC) curves and the area under the curve (AUC), sensitivity, specificity, positive and negative predictive values which were estimated using CART software were used to assess the performance of each CART model.

\subsubsection{Results}

\subsubsection{Sample Characteristics}

There were 602 cadet concussions included in the current sample. Sample characteristics are presented in Table 3.4.1. Of these 602 injuries, $44.02 \%(\mathrm{n}=265)$ occurred during sport, $32.72 \%(\mathrm{n}=197)$ during academy training, $13.12 \%(\mathrm{n}=79)$ during free time, and $10.13 \%(\mathrm{n}=$ 61) were missing an injury activity category. 
Table 3.4.1. Sample Characteristics

\begin{tabular}{|c|c|c|}
\hline Variable & $\%(\mathbf{n})$ & Mean (SD) \\
\hline \multicolumn{3}{|l|}{ Sex } \\
\hline Male & $63.46 \%(382)$ & \\
\hline \multicolumn{3}{|l|}{ Race } \\
\hline White & $68.26 \%(400)$ & \\
\hline African American & $10.24 \%(60)$ & \\
\hline Other & $22.45 \%(135)$ & \\
\hline Missing & $1.19 \%(7)$ & \\
\hline \multicolumn{3}{|l|}{ Sport Level } \\
\hline Varsity & $38.37(265)$ & \\
\hline Club & $19.10 \%(115)$ & \\
\hline Intramural & $40.03 \%(241)$ & \\
\hline Missing & $2.49 \%(15)$ & \\
\hline \multicolumn{3}{|l|}{ Previous Concussion } \\
\hline Yes & $28.57 \%(172)$ & \\
\hline Missing & $4.15 \%(25)$ & \\
\hline Number of Previous Concussions & & $0.40(0.69)$ \\
\hline \multicolumn{3}{|l|}{ Site } \\
\hline 1 & $35.22 \%(212)$ & \\
\hline 2 & $62.79 \%(378)$ & \\
\hline 3 & $1.99 \%(12)$ & \\
\hline Age & & $19.14(1.41)$ \\
\hline Height (m) & & $1.76(0.10)$ \\
\hline Weight (kg) & & $74.78(14.27)$ \\
\hline
\end{tabular}

\subsubsection{Symptoms}

Median symptom severity scores at baseline, $<6$ hours, and 24-48 hours are reported in Table 3.4.2. For cadets who completed both $<6$ hour and 24-48 hour SCAT symptom scales, the presence of each symptom is described in Figure 3.4.1. Headache, pressure in the head, feeling slowed down, and 'don't feel right' were the most common symptoms at $\leq 6$ hours and 24-48 hours. Significant decreases in the proportion of cadets reporting symptoms at the $<6$ hour and 24-48 hour time points were observed for headache, pressure in the head, feeling slowed down, don't feel right, dizziness, feeling like in a fog, drowsiness, balance problems, confusion, nausea or vomiting, blurred vision, nervous or anxious, and sadness (all p's $<0.05$; Figure 3.4.1). Symptoms that remained constant between the $\leq 6$ hour and 24-48 hour time points were difficulty concentrating, fatigue or low energy, sensitivity to light, difficulty remembering, neck pain, sensitivity to noise, more emotional, and irritability (all p's $>0.05$; Figure 3.4.1). Trouble 
falling asleep was the only symptom that increased in prevalence from the $\leq 6$ hours to $24-48$ hour time points.

Table 3.4.2. Median and Interquartile Ranges of Symptom Severity at Baseline, within 6 Hours and 24-48 Hours Post-Concussion

\begin{tabular}{lccc}
\hline Symptom & Baseline & $\mathbf{6 ~ H o u r}$ & $\mathbf{2 4 - 4 8 ~ H o u r ~}$ \\
\hline Headache & $0.00[0.00-0.00]$ & $3.00[2.00-4.00]$ & $2.00[1.00-3.00]$ \\
"Pressure in head" & $0.00[0.00-0.00]$ & $3.00[1.00-4.00]$ & $2.00[1.00-3.00]$ \\
Neck Pain & $0.00[0.00-0.00]$ & $0.00[0.00-2.00]$ & $0.00[0.00-2.00]$ \\
Nausea or vomiting & $0.00[0.00-0.00]$ & $0.00[0.00-2.00]$ & $0.00[0.00-1.00]$ \\
Dizziness & $0.00[0.00-0.00]$ & $1.00[0.00-3.00]$ & $1.00[0.00-2.00]$ \\
Blurred Vision & $0.00[0.00-0.00]$ & $0.00[0.00-1.00]$ & $0.00[0.00-1.00]$ \\
Balance Problems & $0.00[0.00-0.00]$ & $0.00[0.00-1.00]$ & $0.00[0.00-1.00]$ \\
Sensitivity to light & $0.00[0.00-0.00]$ & $1.00[0.00-2.00]$ & $1.00[0.00-2.00]$ \\
Sensitivity to noise & $0.00[0.00-0.00]$ & $0.00[0.00-1.00]$ & $1.00[0.00-2.00]$ \\
Feeling slowed down & $0.00[0.00-1.00]$ & $2.00[0.00-3.00]$ & $1.00[0.00-3.00]$ \\
Feeling like "in a fog" & $0.00[0.00-0.00]$ & $1.00[0.00-3.00]$ & $1.00[0.00-2.00]$ \\
"Don't feel right" & $0.00[0.00-0.00]$ & $2.00[1.00-3.00]$ & $1.00[0.00-3.00]$ \\
Difficulty concentrating & $0.00[0.00-1.00]$ & $1.00[0.00-3.00]$ & $1.00[0.00-3.00]$ \\
Difficulty remembering & $0.00[0.00-0.00]$ & $0.00[0.00-2.00]$ & $0.00[0.00-1.00]$ \\
Fatigue or low energy & $1.00[0.00-3.00]$ & $1.00[0.00-3.00]$ & $1.00[0.00-3.00]$ \\
Confusion & $0.00[0.00-0.00]$ & $0.00[0.00-1.00]$ & $0.00[0.00-1.00]$ \\
Drowsiness & $0.00[0.00-2.00]$ & $1.00[0.00-2.00]$ & $1.00[0.00-2.00]$ \\
Trouble falling asleep & $0.00[0.00-0.00]$ & $0.00[0.00-0.00]$ & $0.00[0.00-1.00]$ \\
More emotional & $0.00[0.00-0.00]$ & $0.00[0.00-0.00]$ & $0.00[0.00-1.00]$ \\
Irritability & $0.00[0.00-0.00]$ & $0.00[0.00-0.00]$ & $0.00[0.00-1.00]$ \\
Sadness & $0.00[0.00-0.00]$ & $0.00[0.00-0.00]$ & $0.00[0.00-0.00]$ \\
Nervous or Anxious & $0.00[0.00-0.00]$ & $0.00[0.00-0.00]$ & $0.00[0.00-0.00]$ \\
\hline
\end{tabular}


Figure 3.4.1. SCAT Symptom Prevalence within 6 and 24-48 Hours Post-Concussion

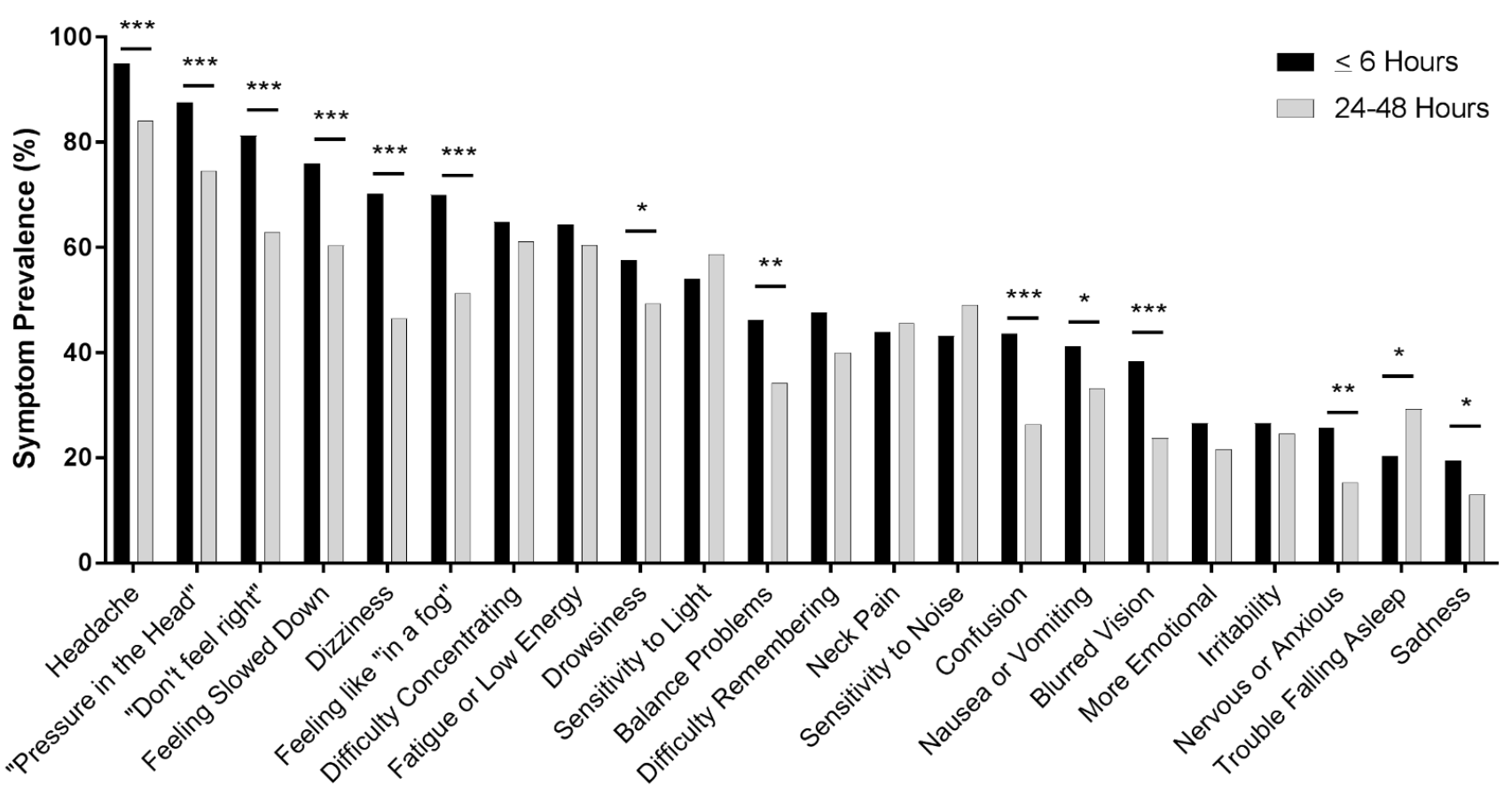

\subsubsection{Normal versus Abnormal Recovery}

CART analyses revealed no significant recovery predictors using SCAT symptom scores or difference scores within 6 hours. Significant recovery predictors were revealed at the 24-48 hour time point using either the SCAT symptom severity scores or difference from baseline.

Using 24-48 hour SCAT symptom scores, balance problems, nausea or vomiting, trouble falling asleep, sadness, and neck pain differentiated between normal and abnormal recovery (Figure 3.4.2). Overall, 88\% $(\mathrm{n}=353)$ of cadets had a normal recovery. Those classified as having poor recovery had greater balance and sadness symptoms (Probability $(\operatorname{Pr})=0.30)$. Also, if a cadet did not report neck pain, but reported low balance problems, high nausea or vomiting, and high trouble sleeping they were classified as having an abnormal recovery $(\operatorname{Pr}=0.29)$ (Figure 3.4.2). Balance problems had the largest effect and had the greatest importance, followed by nausea or vomiting and sadness. The probability for good recovery increased when balance scores were less than two (Probability $(\operatorname{Pr})=0.90)$ and further increased $(\operatorname{Pr}=0.93)$ if nausea or vomiting symptom scores were also less than two (Figure 3.4.2).

Testing the 24-48 hour SCAT symptom score CART model on the testing database yielded 83\% (95\% CI: 0.74-0.90) accuracy. However, the Receiver Operating Characteristic 
(ROC) had an area under the curve (AUC) of 0.55 . The low AUC was generated by the low specificity (0.00 95\% CI: 0.00-0.28) despite high sensitivity (0.93 95\% CI: 0.86-0.98).

Figure 3.4.2. CART Results Categorizing Normal and Abnormal Recovery Using 24-48 Hour Symptom Scores

\section{Balance Problems}

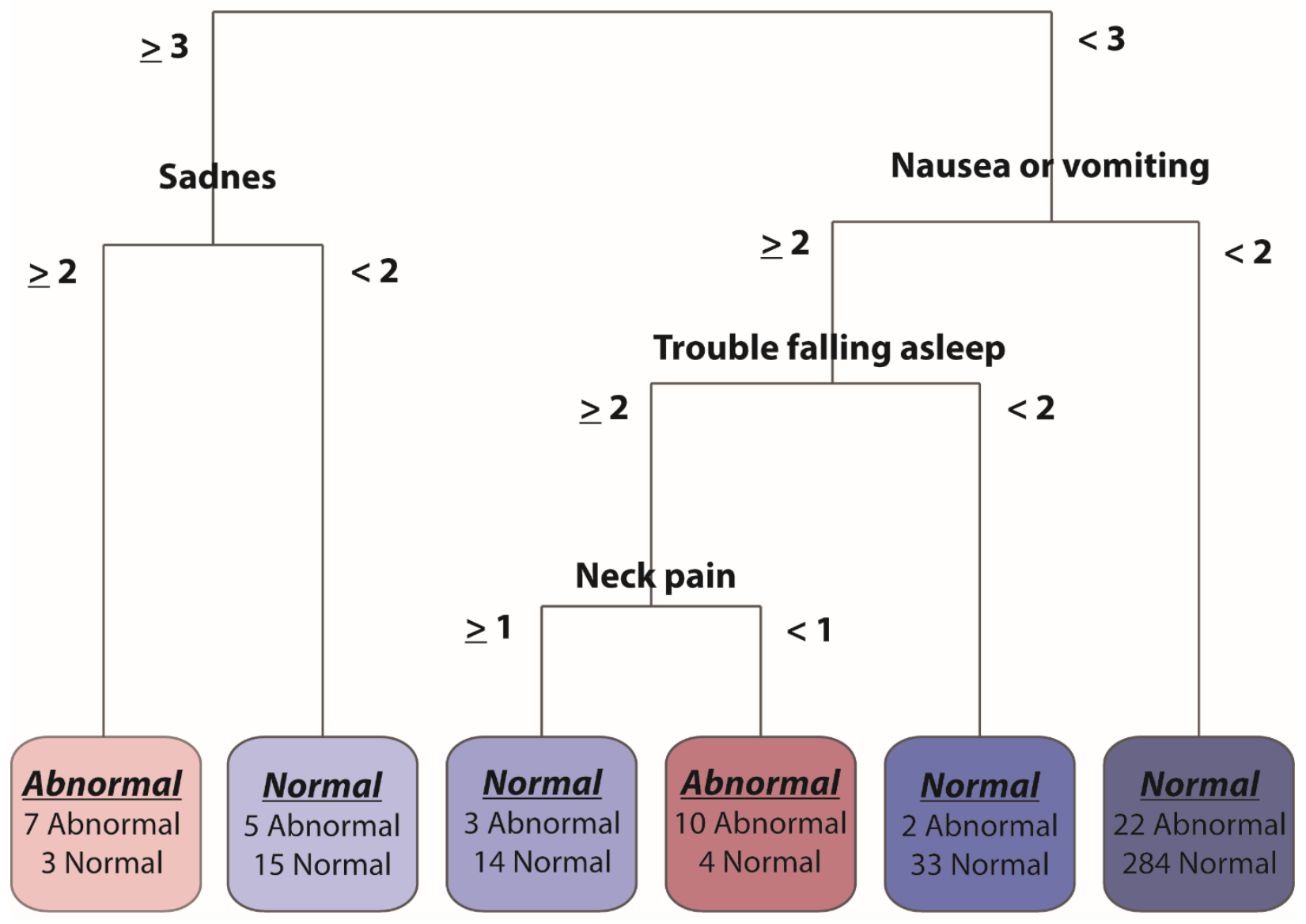

Similarly to the CART analysis of 24-48 hour SCAT symptom scores, the CART analysis of 24-48 hour difference scores yielded similar significant predictors. Using 24-48 hour SCAT difference scores, balance problems, nausea or vomiting, trouble falling asleep, and neck pain differentiated between normal and abnormal recovery (Figure 3.4.3). The only difference between CART models was that sadness in the 24-48 hour symptom score model was replaced by dizziness in the difference score model. 
Cadets were classified as having an abnormal recovery if they reported lower difference scores for neck pain in addition to elevated trouble falling asleep, elevated nausea or vomiting, and decrease balance problem difference scores $(\mathrm{Pr}=0.38)$. Additionally, cadets were also classified as having an abnormal recovery if they experienced elevated balance problems and decreased dizziness symptom difference scores $(\operatorname{Pr}=0.40)$.

Balance problems had the greatest importance, followed by nausea or vomiting and dizziness. If a cadet experienced less than a three-point change in balance problems from baseline the probability for good recovery was $90 \%$. The probability for normal recovery increased when there was less change from baseline symptoms on balance problems and nausea or vomiting $(\operatorname{Pr}=0.93)$ or when there was less deviation from baseline trouble falling asleep symptom score $(\operatorname{Pr}=0.91)$ (Figure 3.4.3).

Testing the difference score CART model on the testing database yielded 85\% (95\% CI: 0.76-0.91) accuracy. The ROC also improved with an AUC of 0.61. The associated specificity improved to $22 \%$ (95\% CI: 0.03-0.60) and sensitivity remained high (0.91 95\% CI: 0.83-0.97). 
Figure 3.4.3. CART Results Categorizing Normal and Abnormal Recovery Using 24-48 Hour Difference Scores

\section{Balance Problems}

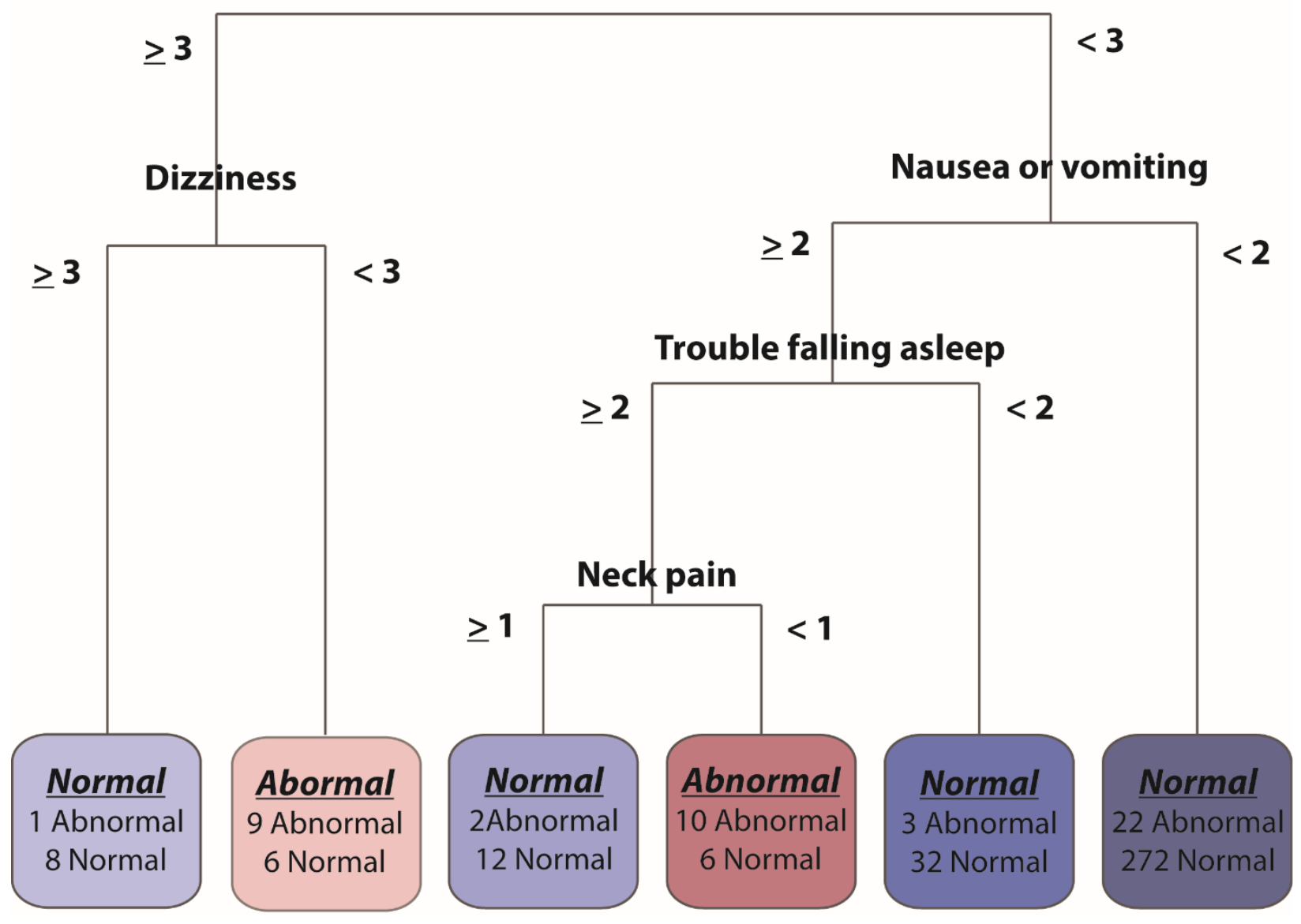

\subsubsection{Discussion}

Results from the current analysis demonstrate three primary findings. First, recovery for a substantial majority of concussed Military Service Academy cadets takes 28 days, rather than 14 days as previously suggested. Secondly, a significant proportion of post-concussion symptoms decrease in prevalence between the $\leq 6$ hour and 24-48 hour time points, indicating symptom evolution. Finally, individual symptoms at 24-48 hours estimate normal and abnormal recovery whereas symptom presentation within 6 hours were not strong estimators of recovery. Furthermore, accounting for baseline symptoms by using difference scores improved the normal and abnormal prediction. Lower symptom reports of balance problems, nausea or vomiting, and trouble falling asleep at 24-48 hours were consistent predictors of normal recovery in both 
models. These results highlight specific individual symptoms that estimate recovery and thus may drive recovery duration.

While the most recent Concussion Consensus Statement defines normal recovery for adults as 14 days (McCrory et al., 2017), the current study demonstrates a significant proportion of concussed individuals remain symptomatic at day 14. Several studies report symptom resolution typically occurring within 7-10 days (McCrea et al., 2005; McCrea et al., 2013; Teel et al., 2017). These studies have been primarily composed of male varsity football athletes and only examined sport-related concussions. The current study enrolled a substantially larger and a more heterogeneous sample of concussed participants that examined both sport and non-sport related concussions. While the current study included non-sport concussions and a more heterogeneous sample, the proportion taking more than 28 days to recover was similar to previous studies (Nelson et al., 2016; Wasserman et al., 2016) suggesting that 28 days a more accurate representation of normal recovery.

Symptom severity decreases within 24-48 hours of concussion (McCrea et al., 2003) and symptom prevalence decreases from $90 \%$ to $25-55 \%$ among concussed participants (McCrea et al., 2005). While overall symptom burden decreases over time, different symptom patterns evolve after a concussion (Marshall et al., 2015). The current study's results support evidence for some symptoms decreasing between $\leq 6$ hours and 24-48 hours, while other symptoms increase. In particular, the most commonly reported symptoms (headache, pressure in head, feeling slowed down) demonstrate significant decreases in individuals experiencing those symptoms. Meanwhile, less common symptoms (difficulty concentrating, sensitivity to light, difficulty remembering) were experienced by a similar proportion of individuals at both time points. Similar to the findings by Marshall and colleagues (Marshall et al., 2015), fatigue and sensitivity to light or sound did not decrease over time. These findings indicate that depending on when an individual was assessed, different symptom profiles were observed. Additionally, these findings support examining individual symptoms along with symptom scores to capture the evolution of symptoms over time.

While $\leq 6$-hour symptoms did not predict recovery, symptom scores and difference scores at 24-48 hours did estimate recovery. In support of our hypothesis, a consistent set of symptoms estimated recovery, including balance problems, nausea or vomiting, and trouble falling asleep. While dizziness and neck pain also estimated normal and abnormal recovery they 
were not in the hypothesized direction. For example, a lower dizziness difference score in the presence of high balance problem difference score was associated with normal recovery. However, further examination of the data revealed that while dizziness difference scores less than three were associated with abnormal recovery, all scores were greater than zero. This indicates that all scores increased from baseline, consistent with greater dizziness symptoms after concussion.

Dizziness has previously been shown to differentiate normal and abnormal recovery (Lau et al., 2011). In Lau and colleagues' study of recovery, dizziness at the time of injury (sideline evaluation) was the strongest and only estimator of normal and abnormal recovery (Lau et al., 2011). Similar to the current study, dizziness was self-reported by the athlete, but unlike the current study baseline dizziness was not accounted for, and the presence of dizziness was dichotomized. Not accounting for baseline dizziness symptoms and categorizing dizziness as present or absent may contribute to the conflicting results. In the current study, increased dizziness difference scores were associated with abnormal recovery, but a symptom score change of three or more was associated with normal recovery. Since $11 \%$ of cadets had an abnormal recovery and only 64 cadets had a dizziness difference score greater or equal to three, only seven cadets would be expected to be classified as having an abnormal recovery. The current CART algorithm may have made the dizziness difference score cutoff due to few cadets having dizziness difference scores greater than three. Consequently, increased dizziness is likely an indicator of abnormal recovery.

Lower neck pain symptoms and difference score were associated with normal recovery. The association between lower neck pain and normal recovery is surprising as neck pain (van der Naalt et al., 2017) and duration of neck pain (Resch et al., 2015) has been associated with worse recovery. The presence of neck pain symptoms may indicate a whiplash-like injury. Over $90 \%$ of whiplash patients reach symptom resolution within 28 days (Partheni et al., 2000). Furthermore, cervicovestibular rehabilitation has been shown to effectively treat neck pain after a concussion (Schneider et al., 2014). Thus, greater acute neck pain symptoms may be classified as normal recovery since they represent a rapidly resolving whiplash injury or a treatable form of neck pain. Future research should examine whether concussion and whiplash injuries are distinct and whether whiplash or concussions with neck pain recover faster than concussions without neck pain. 
The particular symptoms associated with recovery partially supported our other hypothesis that symptoms not commonly reported at baseline would predict recovery. While balance problems and nausea or vomiting are not commonly reported at baseline, trouble falling asleep and neck pain are frequently reported at baseline (Covassin et al., 2006; Piland et al., 2010; Shehata et al., 2009). Further supporting our hypothesis, the symptom difference scores had the best predictive ability, supporting the ongoing use of baseline assessments to facilitate individualized medical care.

The current study is not without limitations. The limited sample size at $\leq 6$ hours likely limited the CART model's ability to identify symptoms associated with recovery. Additionally, the current study defined normal recovery as symptom resolution within 28 days, but depending on the population (child versus adult) and the clinical question, clinicians and patients might be interested in typical recovery within a different time window. Finally, while CART analysis is superior to other prediction models regarding interpretation and ability to handle non-parametric variables, it is also prone to over-fitting. While it is possible the CART models were over-fitted, the current analysis mitigated the likelihood of overfitting by using training and testing datasets.

\subsubsection{Conclusion}

Our findings provide evidence for specific acute symptom profiles to predict normal and abnormal recovery. Increased balance problems, nausea or vomiting, trouble falling asleep and sadness all increased abnormal recovery likelihood. Symptom difference scores, accounting for baseline symptoms, provides the best predictive model in the current study. Thus, baseline symptom evaluation is suggested in order to account for individual level variation in symptom burden. Clinicians and patients should be aware of these symptoms role in recovery. Future research should investigate interventions targeted at these symptoms to improve recovery. 


\subsection{Project 4: Assessing the bidirectional relationship between psychological symptoms and concussion}

\subsubsection{Introduction}

Concussion, or mild traumatic brain injury, is a growing concern for athletes and military service members. 1.6-3.8 million sport or recreation-related concussion occur annually in the United States (Langlois et al., 2006). Concussion is also the signature injury of recent military conflicts, with $15 \%$ of service members classified as having a concussion (Hoge et al., 2008; Wilk et al., 2010). While most concussed individuals will have symptom resolution within 7-10 days post-injury (McCrea et al., 2013), there is growing concern about potential long-term consequences. In particular, mental health disorders, particularly depression (Kerr et al., 2014; Kerr et al., 2012; Meehan et al., 2016), has been associated with concussion years after the injury. However, previous cross-sectional studies finding these associations have not been able to establish temporality; that is if concussion precedes mental health changes or whether mental health changes precede concussion. Studies of all injury, including concussion and musculoskeletal injury, have found that elevated depression and anxiety symptoms increase subsequent injury risk (Li et al., 2017). The objective of the current study is to elucidate the role of psychological symptoms on concussion risk and the role of concussion on subsequent psychological symptoms.

Studies of retired collegiate athletes have found increased anxiety (Meehan et al., 2016) and depression (Kerr et al., 2014; Meehan et al., 2016) symptoms among those with a concussion history. Both retrospective cross-sectional studies found that more previous concussions were associated with elevated depression symptoms (Kerr et al., 2014; Meehan et al., 2016) and one study also found a positive association with anxiety symptoms (Meehan et al., 2016). Each study was conducted using a survey, and response rates ranged from $8-22 \%$ of former collegiate athletes (Kerr et al., 2014; Meehan et al., 2016). Moreover, the studies were limited to a single university (Kerr et al., 2014) or four regional universities (Meehan et al., 2016). Due to the crosssectional design and limited sample, the causal role of concussion and generalizability of findings is unclear. A single prospective cohort study provides stronger causal evidence for concussion's role in mental health. The study of retired National Football League (NFL) players found that a concussion during their NFL career was more likely to result in a subsequent depression diagnosis (Kerr et al., 2012). Moreover, there was a dose-response relationship with 
increasing previous concussions further increasing the depression diagnosis risk (Kerr et al., 2012). While this study establishes that concussion preceded depression diagnosis and that there was a dose-response relationship, it is unknown whether the same relationship is observed in non-NFL players, females, and non-athletes.

In addition to the hypothesized influence of concussion on subsequent mental health, there is growing evidence that mental health symptoms also influence injury risk. A recent study of collegiate athletes found that male and females with increased pre-season anxiety symptoms had a two-fold increase injury rate than athletes without pre-season anxiety symptoms ( $\mathrm{Li}$ et al., 2017). There was no observed effect of depression on injury risk (Li et al., 2017). Notably, injuries included concussions and musculoskeletal injuries. Thus, it is unknown whether anxiety increases all injury risk, or has a particular influence on concussion risk.

In addition to anxiety symptoms, somatization symptoms may also have a role in concussion risk. Somatization symptoms are those symptoms relating to the body (e.g. pain in heart or chest) and have been shown to increase recovery duration by influencing postconcussion symptoms (Nelson et al., 2016). Individuals with greater somatization symptoms may therefore experience post-concussions symptoms to a greater degree. Since a concussion is diagnosed when an individual exhibits symptoms, signs, and behaviors of concussion (McCrory et al., 2017; Putukian, 2011), increased post-concussion symptoms, driven by somatization symptoms, may yield greater diagnoses. Furthermore, stressful life events increase somatization symptoms (Smith, 1990). Since concussion can be a particularly distressing event (Putukian, 2016), we hypothesize that somatization symptoms will increase after a concussive injury.

The objective of the current analysis is to elucidate the role of psychological symptoms on concussion risk and the role of concussion on subsequent psychological symptoms. We hypothesize that anxiety and somatization symptoms will increase concussion risk. Additionally, we hypothesize that concussion will increase subsequent anxiety and depression symptom score.

\subsubsection{Methods}

\subsubsection{Study Design}

The current study is part of a larger joint effort by the U.S. Department of Defense (DoD) and National Collegiate Athletic Association (NCAA). This partnership funded the Concussion, Assessment, Research, and Education (CARE) Consortium, a multi-site investigation on the natural history of concussion. A detailed description of the CARE organization has been 
published previously (Broglio, McCrea, et al., 2017). Site-level Institutional Review Board approval and approval by the DoD Human Research Protections Office were obtained prior to participant consent to the study

Between 2014 and 2017 all cadets at the participating military Service Academies were eligible to enroll in CARE. Before each academic year, each enrolled cadet completed a baseline assessment. Baseline assessments included demographics, medical history, and psychological symptom evaluation using the Brief Symptom Inventory-18 (BSI-18) (Meachen et al., 2008). The BSI-18 is composed of three sub-scores, measuring somatization, depression, and anxiety symptom burdens (Derogatis, 2001). Participants were then monitored by medical staff for concussion defined as a change in brain function following a force to the head, which may or may not be accompanied by temporary loss of consciousness (Carney et al., 2014).

\subsubsection{Statistical Analysis}

The current analyses were limited to cadets with two baseline assessments. If a cadet had more than two baseline assessments, the first two were used. Using the concussion injury date, cadets were classified as having an incident concussion if it was recorded within the CARE study and occurred between the two baselines. Furthermore, cadets were excluded from the analysis if they were missing any of the BSI-18 sub-scales. The resultant database consists of 5034 cadets, from which 214 concussions met the inclusion criteria.

Descriptive statistics were estimated using Chi-Squared tests for nominal variables, independent sample T-tests, and Mann-Whitney U tests. Mann-Whitney U tests were used to compare BSI-18 scores between concussed and non-concussed cadets due to BSI-18's nonnormal distribution.

Structural equation path analyses were performed using RStudio version 3.3.2 using the ‘lavaan’ package (Rosseel, 2012). The hypothesized model (Figure 3.5.1) was defined Year 1injury somatization, depression and anxiety symptoms as independent variables, concussion as the mediator, sex as a confounder, and Year 2-injury somatization, depression and anxiety symptoms as the dependent variables. Two mediation models were generated, one without sex as a covariate and the full model with sex regressed on Year 1 and Year 2 BSI-18 subscales to control for possible confounding effects. 
Figure 3.5.1. Hypothesized Model of Concussion and Psychological Symptoms

\section{Hypothesized Model}

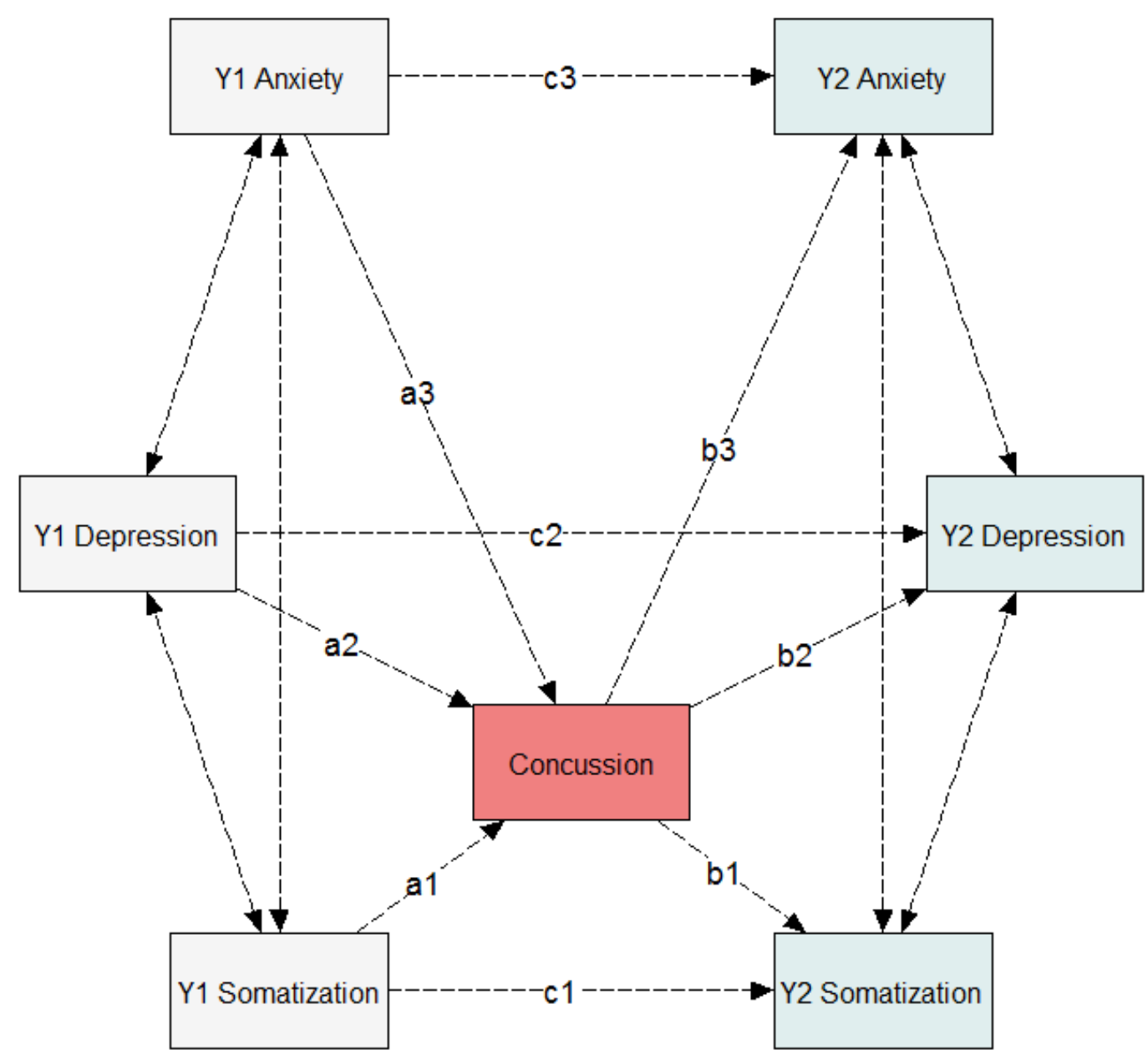

The path model was estimated using weighted least squares, also called asymptotically distribution-free, due to the inclusion of categorical variables (concussion and sex) and nonnormal distribution of BSI-18 sub-scales. Bootstrapped p values of test statistics were estimated from 1000 samples. Model fit was evaluated using Chi-Square $\left(\chi^{2}\right)$, comparative fit index (CFI), root mean square error of approximation (RMSEA), and standardized root mean square residual (SRMR). Thresholds were compared across each model to determine the best fit model. Threshold cutoffs were defined as $\geq 0.90$ for CFI, $<0.08$ for RMSEA, and $<0.10$ for SRMR (Kline, 2015). 
Once the final model was selected a follow-up analysis using the mediation analysis proposed by Iacobucci (2012) (Iacobucci, 2012) was used to confirm the results. The Iacobucci mediation analysis allows for a mix of linear and logistic regressions to assess mediation. This method standardizes each model path so that a mediation effect can be evaluated using a z score. A separate mediation analysis was completed for each BSI-18 subscale. Linear and logistic regression models used the bootstrapping and bias-corrected and accelerated methods. Separate mediation models and descriptive statistical analyses were completed using SPSS v24.

\subsubsection{Results}

\subsubsection{Sample Characteristics}

Of the 5,034 ( $\mathrm{n}=3710$ female) cadets with two annual baseline assessments, $4.25 \%(\mathrm{n}=$ 214) had an incident concussion between baseline assessments. Females had $62 \%$ increased risk of having an incident concussion (95\% CI 1.23-2.14) compared to males (Table 3.5.1).

Additionally, a greater proportion of varsity cadets and cadet with a previous concussion were more likely to have an incident concussion (both p's < 0.01) (Table 3.5.1). Year one baseline somatization symptom scores were significantly greater for the concussed compared to the nonconcussed group $(\mathrm{p}<0.01)$. Year two baseline somatization, depression, and anxiety symptom

scores were significantly greater among those cadets who sustained an incident concussion than those who did not (Table 3.5.1). 
Table 3.5.1. Descriptive Statistics by Incident Concussion

\begin{tabular}{|c|c|c|c|}
\hline & $\begin{array}{c}\text { Concussion } \\
(\mathbf{n}=214)\end{array}$ & $\begin{array}{l}\text { No Concussion } \\
(\mathrm{n}=\mathbf{4 8 2 0})\end{array}$ & $\begin{array}{c}\text { Overall } \\
(\mathbf{N}=\mathbf{5 0 3 4})\end{array}$ \\
\hline Male $^{*}$ & $66.82 \%(143)^{\mathrm{a}}$ & $76.90 \%(3710)$ & $76.5 \%(3853)$ \\
\hline Age & $18.77(1.19)$ & $19.21(1.29)$ & $19.20(1.29)$ \\
\hline Height (m) & $1.76(0.10)$ & $1.77(0.09)$ & $1.77(0.09)$ \\
\hline Weight (kg) & $74.17(14.00)$ & $75.42(12.58)$ & $75.37(12.65)$ \\
\hline \multicolumn{4}{|l|}{ Sport Level } \\
\hline Club & $13.6 \%(29)$ & $16.3 \%(788)$ & $16.2 \%(817)$ \\
\hline Intramural & $46.3 \%(99)$ & $54.7 \%(2635)$ & $54.3 \%(2734)$ \\
\hline Previous Concussion & $30.8 \%(66)^{\mathrm{c}}$ & $19.5 \%(941)$ & $20.0 \%(1007)$ \\
\hline \multicolumn{4}{|l|}{ Year One $¥$} \\
\hline Somatization Symptoms & $42.00[42.00-50.00]^{\mathrm{d}}$ & $42.00[42.00-48.00]$ & $42.00[42.00-48.00]$ \\
\hline Depression Symptoms & $42.00[42.00-45.00]$ & $42.00[42.00-45.00]$ & $42.00[42.00-45.00]$ \\
\hline Anxiety Symptoms & $39.00[39.00-47.00]$ & $39.00[39.00-47.00]$ & $39.00[39.00-47.00]$ \\
\hline \multicolumn{4}{|l|}{ Year Two ${ }^{\ddagger}$} \\
\hline Somatization Symptoms & $42.00[41.00-42.00]^{\mathrm{d}}$ & $42.00[42.00-42.00]$ & $42.00[42.00-42.00]$ \\
\hline Depression Symptoms & $42.00[40.00-42.00]^{\mathrm{d}}$ & $42.00[42.00-42.00]$ & $42.00[42.00-42.00]$ \\
\hline Anxiety Symptoms & $39.00[38.00-39.00]^{\mathrm{d}}$ & $39.00[39.00-39.00]$ & $39.00[39.00-39.00]$ \\
\hline \multicolumn{4}{|c|}{$\begin{array}{l}{ }^{a} \text { Females significantly more likely than males to have an incident concussion } \\
{ }^{b} \text { Varsity cadets significantly more likely than club and intramural cadets to have an incident } \\
\text { concussion } \\
{ }^{c} \text { Cadets with previous concussion significantly more likely to have an incident concussion } \\
{ }^{d} \text { Concussion significantly greater than no concussions } \\
{ }^{*} \text { Denotes Chi-Square Test } \\
¥ \text { Denotes Mann-Whitney U Test }\end{array}$} \\
\hline
\end{tabular}

\subsubsection{Path Analysis}

Path analyses were fit with (Model 1) and without (Model 2) the role of sex as a possible confounder. Model 2 achieved better fit statistics $(\mathrm{CFI}=0.94 ; \mathrm{RMSEA}=0.03 ; \mathrm{SRMR}=0.05)$ (Table 3.5.2). Thus, results from Model 2 are interpreted.

Table 3.5.2. Path Analysis Model Fit Statistics

\begin{tabular}{lcc}
\hline & $\begin{array}{c}\text { Model 1: Sex as a Confounder on } \\
\text { Year 1 and Year 2 BSI-18 Scores }\end{array}$ & Model 2: No Sex Effect \\
\hline Chi-Square & $X^{2}(10)=564.23 ; \mathrm{p}<0.001$ & $X^{2}(6)=26.45 ; \mathrm{p}<0.001$ \\
CFI & 0.36 & 0.94 \\
RMSEA & 0.11 & 0.03 \\
SRMR & 0.30 & 0.05 \\
\hline
\end{tabular}


Path analysis demonstrated a significant partial mediation of somatization by incident concussion. While Year 1 somatization symptoms were positively associated with Year 2 somatization scores $\left(\mathrm{cl}^{\prime}=0.11 ; \mathrm{p}<0.001\right)$, there is also an indirect effect of concussion ( $\mathrm{a} 1^{*} \mathrm{~b} 1=$ $0.01 ; p=0.001)$. Since incident concussion was negatively associated with Year 2 somatization symptoms ( $b 1=-1.22 ; \mathrm{p}<0.0001)$ the indirect effect of concussion reduces the effect of Year 1 somatization on Year 2 somatization symptom scores. Thus, incident concussion has a small protective effect.

While Year 1 depression symptoms $\left(\mathrm{c}^{\prime}{ }^{\prime}=0.16 ; \mathrm{p}<0.001\right)$ and incident concussion ( $\mathrm{b} 2=$ -1.25; $\mathrm{p}<0.001$ ) had direct effects on Year 2 depression symptoms, there was no indirect effect of concussion $(\mathrm{a} 2 * \mathrm{~b} 2=0.01 ; \mathrm{p}=0.67)$. There was no indirect effect since Year 1 depression symptoms were not associated with incident concussion $(p=0.67)$. Year 1 depression symptoms were positively associated with Year 2 depression symptoms (Table 3.5.3). Incident concussion was associated with reduced Year 2 depression symptoms (Table 3.5.3).

The effect of Year 1 anxiety symptoms on Year 2 anxiety symptoms was significantly mediated by incident concussion $(\mathrm{a} 3 * \mathrm{~b} 3=0.01 ; \mathrm{p}=0.04)$. Year 1 anxiety symptom scores were negatively associated with incident concussion $(\mathrm{a} 3=-0.01 ; \mathrm{p}=0.03)$ and incident concussion was negatively associated with Year 2 anxiety symptom scores (b3 = -1.34; $p<0.001)$. Consequently, the small mediating effect of concussion increases Year 2 anxiety symptom scores with increasing Year 1 anxiety symptom scores. 
Table 3.5.3. Path Analysis Unstandardized Parameter Estimates and Standard Errors (SE)

\begin{tabular}{rccc}
\hline Path & Estimate & SE & $\mathrm{p}$ \\
\hline Year 2 Somatization Symptoms & & & $<0.001$ \\
Year 1 Somatization (c1') & 0.11 & 0.01 & $<0.001$ \\
Concussion (b1) & -1.22 & 0.21 & 0.001 \\
Indirect (a1*b1) & $-0.01^{\dagger}$ & $0.01^{\dagger}$ & $<0.001$ \\
Total (c1'+(a1*b1)) & 0.10 & 0.01 & $<0.001$ \\
Year 2 Depression Symptoms & & & $<0.001$ \\
Year 1 Depression (c2') & 0.16 & 0.01 & 0.669 \\
Concussion (b2) & -1.25 & 0.22 & $<0.001$ \\
Indirect (a2*b2) & $0.01^{\dagger}$ & $0.01^{\dagger}$ & $<0.001$ \\
Total (c2' $\left.+\left(a 2^{*} \mathrm{~b} 2\right)\right)$ & 0.16 & 0.01 & $<0.001$ \\
Year 2 Anxiety Symptoms & & & 0.039 \\
Year 1 Anxiety (c3') & 0.11 & 0.01 & $<0.001$ \\
\hline Concussion (b3) & -1.34 & 0.19 & $0.01^{\dagger}$ \\
Indirect (a3*b3) & $0.01^{\dagger}$ & 0.01 & \\
\hline
\end{tabular}

Note: Each path references back to Figure 3.5.1.

$\dagger$ Actual value less than 0.01 , but rounded to two significant digits

Standardized parameter estimates are provided in Figure 3.5.2. Standardized slopes represent the number of standard deviations the outcome changes when the predictor increases by one standard deviation. Additionally, covariance is interpreted as a correlation in the standardized model. Overall, the largest effects are the correlations within BSI-18 subscales at Year 1 and Year 2 (Figure 3.5.2). Year 1 depression is significantly correlated with Year 1 anxiety $(\mathrm{r}=0.68 ; \mathrm{d}=1.85)$ and somatization $(\mathrm{r}=0.55, \mathrm{~d}=1.32)$. Year 1 anxiety is also significantly correlated with Year 1 somatization $(r=0.64 ; d=1.67)$. Similar effects are observed within Year 2. Year 2 depression is significantly correlated with Year 2 anxiety $(r=$ $0.49 ; \mathrm{d}=1.12)$ and somatization $(\mathrm{r}=0.26, \mathrm{~d}=0.54)$. Year 2 anxiety is also significantly correlated with Year 2 somatization $(r=0.30 ; d=0.63)$.

The next largest effect sizes occurred between Year 1 and 2 for each BSI-18 subscale. The smallest effects were observed for Year 1 symptom scores influencing concussion and concussion influencing Year 2 symptom scores. 
Figure 3.5.2. Path Analysis Results in Standardized Parameter Estimates

\section{Standardized Estimates}

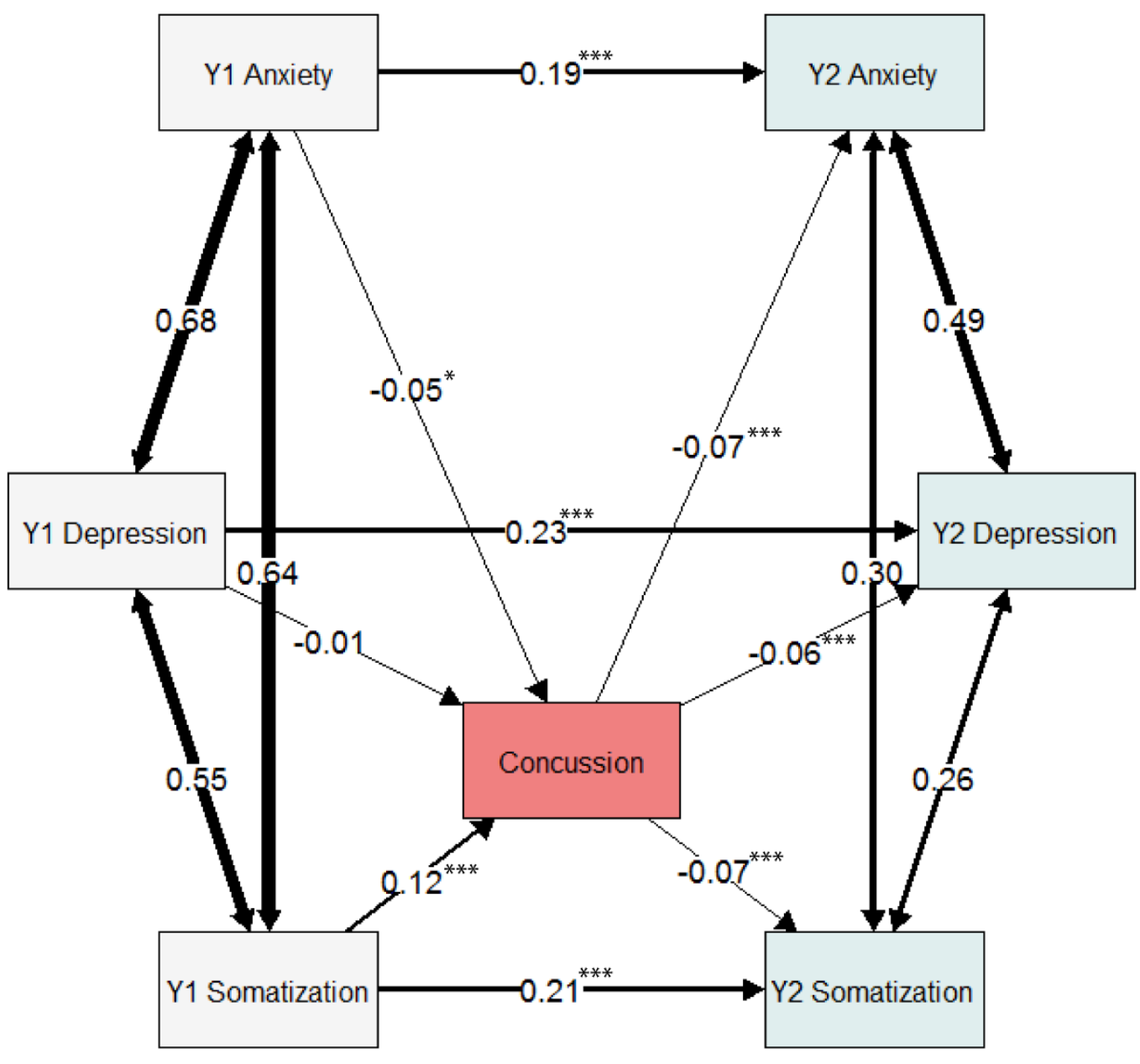

$* * * \mathrm{p}<0.001 ; * * \mathrm{p}<0.01 ; * \mathrm{p}<0.05$

\subsubsection{Mediation Models}

Three mediation models were generated for each BSI-18 symptom to confirm the path analysis results (Iacobucci, 2012). Similar to the path analysis model, incident concussion had a significant partial mediation effect $(Z=-3.51 ; p<0.001)$ (Table 3.5.4). Again, this partial mediating effect was protective, with incident concussion associated with reduced Year 2 somatization symptom scores $(b=-1.15 ; \mathrm{p}=0.001)$. 
There were no significant mediation effects for depression $(Z=-1.22 ; p=0.22)$ or anxiety $(Z=-1.06 ; p=0.29)$ symptoms. Both Year 1 depression $(a=0.01 ; p=0.19)$ and Year 1 anxiety $(\mathrm{a}=0.01 ; \mathrm{p}=0.24)$ symptom scores were not associated with the likelihood for incident concussion. Increasing Year 1 depression $\left(c^{\prime}=0.21 ; p=0.001\right)$ and Year 1 anxiety $\left(c^{\prime}=0.14 ; p\right.$ $=0.001$ ) were directly associated with increased Year 2 depression and anxiety symptom scores, respectively. Incident concussion had a protective effect on Year 2 depression $(b=-1.24 ; p=$ $0.001)$ and Year 2 anxiety $(b=-1.24 ; p=0.001)$ symptom scores. Thus, incident concussion was associated with a 1.24 reduction in depression and anxiety symptom severity scores.

Overall, the Iacobucci method confirmed the path analysis results. The parameter estimates were in the same direction and similar magnitudes. The only difference between methods occurred within anxiety. While the path analysis yielded a significant effect of Year 1 anxiety on concussion the Iacobucci method did find a significant effect. Thus, a significant mediation occurred in the path analysis but not using the Iacobucci method.

Table 3.5.4. Parameter Estimates and Standard Errors (SE) from Iacobucci Mediation Method Model Results

\begin{tabular}{c|cc|cc|cc}
\hline & \multicolumn{2}{|c|}{ Somatization } & \multicolumn{2}{c|}{ Depression } & \multicolumn{2}{c}{ Anxiety } \\
Path & Estimate & SE & Estimate & SE & Estimate & SE \\
\hline $\mathrm{a}$ & 0.04 & 0.01 & 0.01 & 0.01 & 0.01 & 0.01 \\
$\mathrm{c}^{\prime}$ & 0.12 & 0.01 & 0.21 & 0.02 & 0.14 & 0.01 \\
$\mathrm{~b}$ & -1.15 & 0.24 & -1.24 & 0.24 & -1.24 & 0.23 \\
\hline & \multicolumn{2}{|c|}{$\mathrm{Z}=-3.51 ; \mathrm{p}<0.001$} & $\mathrm{Z}=-1.22 ; \mathrm{p}=0.223$ & $\mathrm{Z}=-1.06 ; \mathrm{p}=0.289$ \\
\hline
\end{tabular}

Note: Method from Iacobucci 2012 (Iacobucci, 2012)

\subsubsection{Discussion}

Results from the current study elucidate the significant relationship between concussion and resulting psychological symptoms in addition to the significant impact pre-morbid psychological symptoms have on concussion. Our hypotheses were partially supported by the results. Specifically, Year 1 somatization symptoms had a significant positive association with concussion and Year 1 depression symptoms had no significant relationship with incident concussion. Contrary to our hypotheses, incident concussion had a significant protective effect on Year 2 somatization, depression, and anxiety symptoms. Additionally, Year 1 anxiety symptoms were negatively associated with incident concussion. Overall, results indicate significant bidirectional relationships between psychological symptoms and concussion. 
Increasing baseline somatization symptoms have been associated with increased postconcussion symptoms within 24 hours of injury (Nelson et al., 2016). Based on this finding, we hypothesized that increased Year 1 somatization symptoms would have a significant positive association with incident concussion. Our hypothesis was supported by the significant positive association observed between Year 1 somatization symptoms and subsequent concussion. Somatization symptoms may have a positive relationship with concussion by elevating the symptom response to a concussive injury (Nelson et al., 2016). Thus, more concussions might be diagnosed among individuals with greater baseline somatization. Future studies should test this hypothesized mechanism by evaluating baseline somatization symptoms, concussion, the clinician's confidence in diagnosis (Kutcher \& Giza, 2014), and whether symptom burden was the primary evidence for concussion diagnosis.

Year 2 somatization symptoms were also significantly influenced by concussion. Surprisingly, concussion was associated with fewer somatization symptoms at the Year 2 baseline. While concussion can be a stressful event (Putukian, 2016) and somatization symptoms increase after a stressful life event (Smith, 1990), it appears that concussion does not influence somatization symptoms. It is also possible that concussion only has an immediate impact on somatization symptoms that is resolved and thus unobservable by the time the cadet is evaluated at their next annual evaluation. Future studies should examine somatization symptoms in the acute and sub-acute period after a concussion to determine whether concussion had a more immediate impact on somatization symptoms.

Depression symptoms (Kerr et al., 2014; Meehan et al., 2016) and diagnosis (Kerr et al., 2012) have been the most commonly investigated aspect of mental health after a concussion. While previous studies have found elevated depression symptoms among retired collegiate athletes with a concussion (Kerr et al., 2014; Meehan et al., 2016), the current study found that concussion was associated with lower depression symptoms at the next annual evaluation. However, the previous studies had low survey response rates (8-22\%) and did not account for pre-concussion depression symptoms (Kerr et al., 2014; Meehan et al., 2016). While low response rates do not directly create selection bias, they do increase the opportunity for selection bias (Sneyd \& Cox, 2011). If individuals were more likely to participate in the studies because they are depressed, then results might be biased towards a significant finding. Additionally, while the current study accounted for baseline depression symptoms, the previous studies were 
not able to due to their retrospective design. Thus, as shown in the current study, subsequent depression symptoms might be primarily driven by pre-morbid depression symptoms. Alternatively, the effect of concussion on depression symptoms may evolve over time. Previous studies evaluated depression symptoms years (15-20 years) after an individual's collegiate career (Kerr et al., 2014; Meehan et al., 2016). The current study evaluated depression symptoms within a year of a concussive injury, possibly not leaving time for depression symptoms to evolve. Future studies should prospectively evaluate the effect of concussion on depression years after injury while controlling for pre-morbid depression symptoms.

The current study also found no significant relationship between Year 1 depression symptoms and incident concussion. This result supports previous research finding no relationship between baseline depressive symptoms and any type of injury risk (Li et al., 2017). This finding adds to the current literature by further specifying that there is no concussion-specific risk relating to baseline depression symptoms.

Similar to depression, anxiety symptoms have also been shown to be elevated among retired collegiate athletes with concussion (Meehan et al., 2016). Interestingly, the current study found a protective effect of concussion. Whereby concussion was associated with lower anxiety symptoms at the Year 2 evaluation. Again, the previous study may have been plagued by selection bias and did not account for pre-morbid anxiety symptoms (Meehan et al., 2016). As discussed above, these may contribute to the conflicting results with the current study. Previous research has also identified anxiety symptoms as increasing any concussion risk (Li et al., 2017). While the current study found a significant relationship between Year 1 anxiety symptoms and concussion risk, it was not in the hypothesized direction. Consequently, anxiety symptoms may only increase musculoskeletal injury risk (Li et al., 2017). Also, the current study accounted for correlations between baseline somatization, depression, and anxiety symptoms.

The current study is limited by not only evaluating psychological symptoms at serial baseline assessments. Being able to evaluate psychological symptoms during acute and sub-acute post-concussion phases would elucidate the more immediate effect of concussion on psychological symptoms. Future studies should enroll a control group, without an incident concussion, and compare psychological symptoms profiles to concussed individuals and similar time points post-concussion. Additionally, while every effort was made to capture every incident concussion, it is possible concussions were not reported and this not included in the analysis. If a 
significant proportion of cadets concealed their concussive injuries, it could bias the results. However, since concussion risks and rates among cadets (Chapter 3.2) (O’Connor in prep) are similar to previously reported collegiate concussion risks and rates (Wasserman et al., 2016), it is unlikely that there is a disproportionate amount of non-reporting. Finally, somatization symptoms are non-specific and may also occur in depression and anxiety disorders (Henningsen, Zimmermann, \& Sattel, 2003; Kapfhammer, 2006). Thus, somatization symptoms experienced by cadets may overlap with depression or anxiety. Further work is needed to understand the generators of somatic complaints and their relationship to concussion risk and outcomes.

\subsubsection{Conclusion}

The current study is the first to evaluate somatization, depression, and anxiety symptoms on incident concussion risk. Somatization was the only psychological domain associated with increased incident concussion risk. Results may indicate that somatization symptoms, rather than depression or anxiety symptoms influence concussion risk. While the directions of relationships were not all as expected, results indicate that there is a bidirectional relationship between psychological symptoms and concussion. Specifically, somatization may have the greatest impact on concussion risk. Previous studies finding associations between anxiety and any injury risk might be indirectly measuring somatization symptoms due to the strong correlation between anxiety and somatization symptoms. Future studies should evaluate somatization, depression, and anxiety symptoms to validate the current results. 


\section{CHAPTER 4: Summary and Future Directions}

\subsection{Summary}

Concussion results from heterogeneous mechanisms and has widespread burden. Fortytwo million individuals across the globe are affected by concussion annually (Cassidy et al., 2004). Individual sustain concussions due to sport or recreation-related activities (Langlois et al., 2006), falls, motor vehicle accidents (Cameron et al., 2012; Nelson et al., 2018), or combatrelated activities (Cameron et al., 2012). The results from the current dissertation's projects identifies cohorts of cadets at increased risk for concussion, increased risk for prolonged recovery, and elucidates the relationship between psychological symptoms and concussion. The prospective study design, inclusion of females and multiple concussive injury mechanisms adds to the current understanding by enabling a multivariate approach to identify individual risk factors and better generalization of findings.

Individual risk factors for concussion included female sex, varsity athlete status, freshmen, having a previous concussion, headaches, ADD/ADHD, and greater BSI-18 somatization symptoms. Each of these factors were associated with a 1.02-2.73 increased concussion risk. Similar risk factors were observed for concussions caused by sport or academy training-related activities. Free time-related concussions were only associated with female sex, freshmen, and previous concussion. These results indicate that females, freshmen, and those with a previous concussion are at greatest risk for an incident concussion of any type. Previous studies 
were limited by only studying sport-related concussions (Zuckerman et al., 2015) or those concussions presenting to the emergency department (Bazarian et al., 2005). Using a single population of U.S. Service Academy cadets, a more comprehensive risk profile for concussion has been generated.

Separate factors were associated with symptom duration and RTA protocol duration. Thus, symptom duration and RTA protocol durations are likely related but separate entities within total time loss after a concussion. Specifically, the acute number of symptoms had the most consistent and largest effect on recovery durations. Examining individual symptoms, the symptom difference scores between baseline and 24-48 hours post-concussion best predicted normal versus abnormal recovery. Specific symptoms classifying normal versus abnormal recovery were balance problems, dizziness, trouble falling asleep, nausea, and neck pain. The individual symptoms may represent a more informative symptom profile related to concussion recovery and also points of clinical intervention.

Finally, the relationship between psychological symptoms and concussion was examined. Most studies have focused on a concussion's effect on subsequent mental health (Kerr et al., 2014; Kerr et al., 2012). However, it is also possible that mental health factors, specifically depression and anxiety, influence concussion risk (Li et al., 2017). Results demonstrated that baseline somatization had the strongest association with subsequent concussion and future somatization symptoms. Moreover, an incident concussion was associated with lower depression, anxiety, and somatization symptoms, albeit the size of these effects were small. Overall, results indicate a bidirectional relationship between concussion and psychological symptoms which encourages future studies to account of baseline psychological symptoms or mental health.

\subsection{Limitations}

Since the current series of dissertation projects focused on U.S. Service Academy cadets, it is unclear how generalizable the results are. While a greater proportion of females were included in the current sample and all concussion types were evaluated, cadets are an extremely healthy and driven population. While cadets are devoid of many medical comorbidities associated with concussion, facilitating research to understand the singular influence of concussion, generalizability may be limited. It is likely that results are more generalizable to healthier athletic populations versus non-athletic populations who may present to emergency 
departments. While efforts were made to prevent over fitting models to the current sample, validation and replication of these results will be critical to confirm results.

\subsection{Future Directions}

Future studies should continue to utilize multivariate risk models. Many results in the current study conflict with previous studies examining singular group level variables. The current series of projects indicate that a constellation of factors are likely related to concussion risk, recovery, and outcomes. Adequately powered longitudinal studies should also be undertaken to understand the long term effects of concussion. Finally, studies should attempt to validate and replicate the findings in the current dissertation to determine the generalizability and consistency of findings.

One of the major findings was the role of symptoms on recovery. Greater symptoms postconcussion, and certain constellations of symptoms were associated with prolonged recovery. Future research should re-address the development of the concussion symptom assessment. First, researchers should determine whether the order of symptom presentation influences symptom prevalence. Anecdotally, individuals more diligently answer symptoms presented first and then circle a single score for the remaining items. Furthermore, looking across symptom scales, symptoms presented earlier on the scale tend to have greater prevalence (Asken, Snyder, et al., 2017). Thus, to identify which symptoms concussion influences, researchers must better characterize symptom prevalence pre and post-concussion. Once symptom prevalence is established, the test-retest reliability of each individual symptom can be evaluated.

Understanding the variability in symptom presence and severity over time will provide the information necessary to establish reliable change indices. These indices will help determine the normal variation associated with a symptom and what change in symptom severity indicates is indicative of a concussion. With the knowledge gained from the symptom prevalence and testretest studies, the next step would be to use the symptom scale as a diagnostic tool. Ideally, a study using longitudinal assessments of concussion symptoms and two control groups (healthy and orthopedic injury) would test whether there are certain symptoms indicative of concussion. This refined symptom tool could be used as part of the clinical assessment post-concussion and would provide the clinician with a better assessment of concussion-related rather than general injury-related symptoms. 


\subsection{Conclusions}

The current series of dissertation projects addresses factors associated with concussion risk and recovery. Individual characteristics including female sex, varsity athletes, previous concussion, headache, ADD/ADHD diagnosis, and increasing somatization symptoms were associated with increased concussion risk. When assessing concussion recovery, two recovery constructs emerged: the duration until asymptomatic and the RTA protocol duration. Symptom duration was influenced by varsity status and elevated symptoms at the time of concussion. Furthermore, certain symptoms were indicative of prolonged recovery. RTA protocol duration was also influenced by varsity status and elevated symptoms at the time of concussion, but also site. Finally, concussion significantly mediated the relationship between subsequent measures of anxiety and somatization symptoms indicating that concussion not only influences psychiatric symptoms, but that psychiatric symptoms influence concussion risk. Overall, these results identify cohorts who are susceptible to concussion and poor concussion-related outcomes. 


\section{APPENDIX}

\section{Appendix A: Model Selection Tables Project 1}

Supplementary Table 3.2.1. Univariate Associations with Sex

\begin{tabular}{|c|c|c|c|c|}
\hline & Female & Male & Test & Effect Size \\
\hline \multicolumn{5}{|l|}{ Sport Level } \\
\hline Varsity & $28.31 \%(830)$ & $71.69 \%(2102)$ & \multirow{3}{*}{$X^{2}(2)=118.14 ; p<0.001$} & \multirow{3}{*}{$\Phi=0.11$} \\
\hline Club & $29.58 \%(551)$ & $70.42 \%(1312)$ & & \\
\hline Intramural & $19.61 \%(1045)$ & $80.39 \%(4285)$ & & \\
\hline \multicolumn{5}{|l|}{ Varsity Contact Level^ ${ }^{\wedge}$} \\
\hline Contact & $20.08 \%(359)$ & $79.92 \%(1429)$ & \multirow{3}{*}{$X^{2}(2)=138.14 ; p<0.001$} & \multirow{3}{*}{$\Phi=0.22$} \\
\hline Limited-Contact & $41.62 \%(154)$ & $58.38 \%(216)$ & & \\
\hline Non-Contact & $39.64 \%(283)$ & $60.36 \%(431)$ & & \\
\hline \multicolumn{5}{|l|}{ Freshman } \\
\hline Yes & $26.67 \%(735)$ & $73.33 \%(2021)$ & \multirow{2}{*}{$\begin{array}{c}\mathrm{X}^{2}(1)=14.80 ; \mathrm{p}<0.001 \\
\text { OR: } 0.1 .22 \text { (95\% CI: } 1.10- \\
1.35)\end{array}$} & \multirow[b]{2}{*}{$\mathrm{d}=0.11$} \\
\hline No & $23.00 \%(1694)$ & $77.00 \%(5671)$ & & \\
\hline \multicolumn{5}{|l|}{ Site } \\
\hline 1 & $20.46 \%(931)$ & $79.54 \%(3619)$ & \multirow{3}{*}{$\mathrm{X}^{2}(2)=117.91 ; \mathrm{p}<0.001$} & \multirow{3}{*}{$\Phi=0.11$} \\
\hline 2 & $24.84 \%(1162)$ & $75.16 \%(3516)$ & & \\
\hline 3 & $36.94 \%(341)$ & $63.06 \%(582)$ & & \\
\hline \multicolumn{5}{|l|}{ Previous Concussion } \\
\hline Yes & $21.20 \%(425)$ & $78.80 \%(1580)$ & $X^{2}(1)=10.73 ; p<0.01$ & \multirow{2}{*}{$\mathrm{d}=0.11$} \\
\hline No & $24.69 \%(1982)$ & $75.31 \%(6046)$ & OR: 0.82 (95\% CI: 0.73-0.92) & \\
\hline \multicolumn{5}{|l|}{$\begin{array}{l}\text { Headaches in past } 3 \\
\text { months }\end{array}$} \\
\hline Yes & $33.69 \%(756)$ & $66.31 \%(1488)$ & $X^{2}(1)=146.93 ; p<0.001$ & \multirow{2}{*}{$\mathrm{d}=0.35$} \\
\hline No & $21.26 \%(1642)$ & $78.74 \%(6080)$ & OR: 1.88 (95\% CI: 1.70-2.09) & \\
\hline \multicolumn{5}{|l|}{$\begin{array}{l}\text { Diagnosed Migraine } \\
\text { Headache }\end{array}$} \\
\hline Yes & $34.65 \%(88)$ & $65.35 \%(166)$ & $X^{2}(1)=15.94 ; p<0.001$ & \multirow{2}{*}{$\mathrm{d}=0.30$} \\
\hline No & $23.80 \%(2317)$ & $76.20 \%(7420)$ & OR: 1.70 (95\% CI: 1.31-2.21) & \\
\hline Diagnosed ADD/ADHD & & & & \\
\hline
\end{tabular}




$$
\begin{array}{ccccc}
\text { Yes } & 17.27 \%(24) & 82.73 \%(115) & X^{2}(1)=3.63 ; p=0.06 & \Phi=0.02 \\
\text { No } & 24.23 \%(2378) & 75.77 \%(7436) & \text { OR: } 0.65(95 \% \text { CI: } 0.42-1.02) & \mathrm{d}=0.24
\end{array}
$$

Diagnosed Depression

$\begin{array}{rcccc}\text { Yes } & 50.96 \%(53) & 49.04 \%(51) & \mathrm{X}^{2}(1)=41.55 ; \mathrm{p}<0.001 & \Phi=0.06 \\ \text { No } & 23.79 \%(2353) & 76.21 \%(7536) & \text { OR: } 3.33(95 \% \text { CI: } 2.26-4.90) & \mathrm{d}=0.66 \\ \begin{array}{l}\text { Brief Sensation Seeking } \\ \text { Scale }\end{array} & 3.23(0.67) & 3.46(0.68) & \mathrm{T}(9520)=-13.71 ; \mathrm{p}<0.001 & \mathrm{~d}=0.32\end{array}$

SCAT Symptoms

BSI Total

$$
\text { Number }
$$

$$
\begin{gathered}
2.00[0.00- \\
5.00] \\
3.00[0.00- \\
8.00] \\
39.00[33.00- \\
47.00] \\
41.00[41.00- \\
48.00] \\
40.00[40.00- \\
45.00] \\
38.00[38.00- \\
45.00]
\end{gathered}
$$$$
\text { Somatization }
$$

Depression

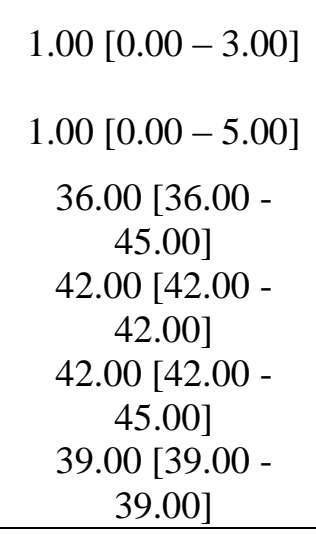

$$
\begin{aligned}
& d=0.27 \\
& d=0.27 \\
& d=0.28 \\
& d=0.61 \\
& d=0.83 \\
& d=0.74
\end{aligned}
$$

$\dagger$ Satterthwaite independent t-test

$¥$ Wilcoxon two-sample test, medians and interquartile range reported

$\wedge$ Within varsity level athletes only 
Supplementary Table 3.2.2a. Mixed Model Selection - Any Concussion

\begin{tabular}{|c|c|c|c|c|c|c|c|c|c|c|c|c|}
\hline & Model 1 & Model 2 & Model 3 & Model 4 & Model 5 & Model 6 & Model 7 & Model 8 & Model 9 & Model 10 & Model 11 & Model 12 \\
\hline & $\mathrm{P}<$ & $\mathrm{P}<$ & $\mathrm{P}<$ & $\mathrm{P}<$ & $\mathrm{P}<$ & $\mathrm{P}<$ & $\mathrm{P}<$ & $\mathrm{P}<$ & $\mathrm{P}<$ & $\mathrm{P}<$ & $\mathrm{P}<$ & \multirow{2}{*}{$\mathrm{P}<0.0001$} \\
\hline Sex & 0.0001 & 0.0001 & 0.0001 & 0.0001 & 0.0001 & 0.0001 & 0.0001 & 0.0001 & 0.0001 & 0.0001 & 0.0001 & \\
\hline & & $\mathrm{P}<$ & $\mathrm{P}<$ & $\mathrm{P}<$ & $\mathrm{P}<$ & $\mathrm{P}<$ & $\mathrm{P}<$ & $\mathrm{P}<$ & $\mathrm{P}<$ & $\mathrm{P}<$ & $\mathrm{P}<$ & \multirow{2}{*}{$\mathrm{P}<0.0001$} \\
\hline Sport Level & & 0.0001 & 0.0001 & 0.0001 & 0.0001 & 0.0001 & 0.0001 & 0.0001 & 0.0001 & 0.0001 & 0.0001 & \\
\hline & & & $\mathrm{P}<$ & $\mathrm{P}<$ & $\mathrm{P}<$ & $\mathrm{P}<$ & $\mathrm{P}<$ & $\mathrm{P}<$ & $\mathrm{P}<$ & $\mathrm{P}<$ & $\mathrm{P}<$ & \multirow{2}{*}{$\mathrm{P}<0.0001$} \\
\hline Freshman & & & 0.0001 & 0.0001 & 0.0001 & 0.0001 & 0.0001 & 0.0001 & 0.0001 & 0.0001 & 0.0001 & \\
\hline & & & & $\mathrm{P}<$ & $\mathrm{P}<$ & $\mathrm{P}<$ & $\mathrm{P}<$ & $\mathrm{P}<$ & $\mathrm{P}<$ & $\mathrm{P}<$ & $\mathrm{P}<$ & \multirow{2}{*}{$\mathrm{P}<0.0001$} \\
\hline Previous Concussion & & & & 0.0001 & 0.0001 & 0.0001 & 0.0001 & 0.0001 & 0.0001 & 0.0001 & 0.0001 & \\
\hline Headache in the past & & & & & $\mathrm{P}<$ & $\mathrm{P}<$ & $\mathrm{P}<$ & $\mathrm{P}<$ & $\mathrm{P}<$ & $\mathrm{P}<$ & $\mathrm{P}<$ & \multirow{2}{*}{$P<0.0001$} \\
\hline three months & & & & & 0.0001 & 0.0001 & 0.0001 & 0.0001 & 0.0001 & 0.0001 & 0.0001 & \\
\hline Migraine Headache & & & & & & $\mathrm{p}=0.22$ & & & & & & \\
\hline $\mathrm{ADD} / \mathrm{ADHD}$ & & & & & & & $\mathrm{p}<0.01$ & $\mathrm{p}=0.01$ & $\mathrm{p}<0.01$ & $\mathrm{p}<0.01$ & $\mathrm{p}<0.01$ & $\mathrm{p}<0.01$ \\
\hline Depression & & & & & & & & $\mathrm{p}=0.10$ & & & & \\
\hline BSI Somatization & & & & & & & & & $\begin{array}{c}\mathrm{p}< \\
0.001\end{array}$ & $\mathrm{p}<0.001$ & $\mathrm{p}=0.08$ & $\mathrm{p}=0.02$ \\
\hline BSI Depression & & & & & & & & & $\mathrm{p}=0.30$ & & & \\
\hline BSI Anxiety & & & & & & & & & $\mathrm{p}=0.38$ & & & \\
\hline SCAT Severity Score & & & & & & & & & & & $\mathrm{p}=0.18$ & \\
\hline BSSS & & & & & & & & & & & & $\mathrm{p}=0.95$ \\
\hline -2logLikelihood & 4755.67 & 4714.51 & 4527.09 & 4468.74 & 4441.88 & 4440.47 & 4435.24 & 4432.82 & 4397.95 & 4406.3 & 4399.13 & 4252.05 \\
\hline $\mathrm{BIC}$ & 4758.96 & 4720 & 4533.68 & 4476.43 & 4450.67 & 4450.36 & 4445.13 & 4443.81 & 4411.14 & 4417.29 & 4411.21 & 4264.14 \\
\hline
\end{tabular}


Supplementary Table 3.2.2b. Mixed Model Selection - Any Sport-Related Concussion

\begin{tabular}{|c|c|c|c|c|c|c|c|c|c|c|c|c|}
\hline & $\begin{array}{c}\text { Model } \\
1\end{array}$ & Model 2 & $\begin{array}{l}\text { Model } \\
3\end{array}$ & $\begin{array}{l}\text { Model } \\
4\end{array}$ & $\begin{array}{l}\text { Model } \\
5\end{array}$ & $\begin{array}{l}\text { Model } \\
6\end{array}$ & $\begin{array}{c}\text { Model } \\
7\end{array}$ & $\begin{array}{c}\text { Model } \\
8\end{array}$ & Model 9 & Model 10 & Model 11 & Model 12 \\
\hline Sex & $\begin{array}{c}\mathrm{p}< \\
0.0001\end{array}$ & $\mathrm{p}<0.01$ & $\begin{array}{l}\mathrm{p}< \\
0.01\end{array}$ & $\begin{array}{l}\mathrm{p}< \\
0.01\end{array}$ & $\begin{array}{l}\mathrm{p}< \\
0.01\end{array}$ & $\begin{array}{l}\mathrm{p}< \\
0.01\end{array}$ & $\begin{array}{l}\mathrm{p}< \\
0.01\end{array}$ & $\begin{array}{l}\mathrm{p}< \\
0.01\end{array}$ & $\mathrm{p}<0.01$ & $\mathrm{p}=0.01$ & $\mathrm{p}=0.01$ & $\mathrm{p}<0.01$ \\
\hline Sport Level & & $\mathrm{p}<0.0001$ & $\begin{array}{c}\mathrm{p}< \\
0.0001\end{array}$ & $\begin{array}{c}\mathrm{p}< \\
0.0001\end{array}$ & $\begin{array}{c}\mathrm{p}< \\
0.0001\end{array}$ & $\begin{array}{c}\mathrm{p}< \\
0.0001\end{array}$ & $\begin{array}{c}\mathrm{p}< \\
0.0001\end{array}$ & $\begin{array}{c}\mathrm{p}< \\
0.0001\end{array}$ & $\mathrm{p}<0.0001$ & $\mathrm{p}<0.0001$ & $\mathrm{p}<0.0001$ & $\mathrm{p}<0.0001$ \\
\hline Freshman & & & $\begin{array}{c}\mathrm{p}< \\
0.0001\end{array}$ & $\begin{array}{c}\mathrm{p}< \\
0.0001\end{array}$ & $\begin{array}{c}\mathrm{p}< \\
0.0001\end{array}$ & $\begin{array}{c}\mathrm{p}< \\
0.0001\end{array}$ & $\begin{array}{c}\mathrm{p}< \\
0.0001\end{array}$ & $\begin{array}{c}\mathrm{p}< \\
0.0001\end{array}$ & $\mathrm{p}<0.0001$ & $\mathrm{p}<0.001$ & $\mathrm{p}<0.001$ & $\mathrm{p}<0.01$ \\
\hline Previous Concussion & & & & $\begin{array}{c}\mathrm{p}< \\
0.0001\end{array}$ & $\begin{array}{c}\mathrm{p}< \\
0.0001\end{array}$ & $\begin{array}{c}\mathrm{p}< \\
0.0001\end{array}$ & $\begin{array}{c}\mathrm{p}< \\
0.0001\end{array}$ & $\begin{array}{c}\mathrm{p}< \\
0.0001\end{array}$ & $\mathrm{p}<0.0001$ & $\mathrm{p}<0.0001$ & $\mathrm{p}<0.0001$ & $\mathrm{p}<0.0001$ \\
\hline $\begin{array}{l}\text { Headache in the past } \\
\text { three months }\end{array}$ & & & & & $\begin{array}{c}\mathrm{p}< \\
0.0001\end{array}$ & $\begin{array}{c}\mathrm{p}< \\
0.0001 \\
\mathrm{p}=\end{array}$ & $\begin{array}{c}\mathrm{p}< \\
0.0001\end{array}$ & $\begin{array}{c}\mathrm{p}< \\
0.0001\end{array}$ & $\mathrm{p}<0.0001$ & $\mathrm{p}<0.01$ & $\mathrm{p}<0.01$ & $\mathrm{p}<0.01$ \\
\hline Migraine Headache & & & & & & 0.26 & & & & & & \\
\hline $\mathrm{ADD} / \mathrm{ADHD}$ & & & & & & & $\begin{array}{l}\mathrm{p}= \\
0.73\end{array}$ & & & & & \\
\hline Depression & & & & & & & & $\begin{array}{l}\mathrm{p}= \\
0.56\end{array}$ & & & & \\
\hline BSI Somatization & & & & & & & & & $\mathrm{p}=0.02$ & $\mathrm{p}<0.01$ & $\mathrm{p}=0.11$ & $\mathrm{p}=0.01$ \\
\hline BSI Depression & & & & & & & & & $\mathrm{p}=0.86$ & & & \\
\hline BSI Anxiety & & & & & & & & & $\mathrm{p}=0.66$ & & & \\
\hline $\begin{array}{l}\text { SCAT Severity Score } \\
\text { BSSS }\end{array}$ & & & & & & & & & & & $\mathrm{p}=0.51$ & $\mathrm{n}=0.69$ \\
\hline -2logLikelihood & 2670.75 & 2515.52 & 2490.17 & 2459.11 & 2444.97 & 2443.8 & 2444.86 & 2444.67 & 2422.16 & 2422.59 & 2416.12 & 2358.29 \\
\hline $\mathrm{BIC}$ & 2674.05 & 2521.01 & 2496.76 & 2466.8 & 2453.76 & 2453.69 & 2454.75 & 2454.55 & 2434.25 & 2432.48 & 2427.1 & 2369.27 \\
\hline
\end{tabular}


Supplementary Table 3.2.2c. Mixed Model Selection - Any Academy Training-Related Concussion

\begin{tabular}{|c|c|c|c|c|c|c|c|c|c|c|c|c|}
\hline & $\begin{array}{c}\text { Model } \\
1\end{array}$ & $\begin{array}{c}\text { Model } \\
2\end{array}$ & $\begin{array}{c}\text { Model } \\
3\end{array}$ & $\begin{array}{c}\text { Model } \\
4\end{array}$ & $\begin{array}{c}\text { Model } \\
5\end{array}$ & $\begin{array}{c}\text { Model } \\
6\end{array}$ & $\begin{array}{c}\text { Model } \\
7\end{array}$ & $\begin{array}{c}\text { Model } \\
8\end{array}$ & Model 9 & Model 10 & Model 11 & $\begin{array}{c}\text { Model } \\
12\end{array}$ \\
\hline Sex & $\begin{array}{c}\mathrm{p}< \\
0.0001\end{array}$ & $\begin{array}{c}\mathrm{p}< \\
0.0001\end{array}$ & $\begin{array}{c}\mathrm{p}< \\
0.0001\end{array}$ & $\begin{array}{c}\mathrm{p}< \\
0.0001\end{array}$ & $\begin{array}{c}\mathrm{p}< \\
0.0001\end{array}$ & $\begin{array}{c}\mathrm{p}< \\
0.0001\end{array}$ & $\begin{array}{c}\mathrm{p}< \\
0.0001\end{array}$ & $\begin{array}{c}\mathrm{p}< \\
0.0001\end{array}$ & $\mathrm{p}<0.0001$ & $\mathrm{p}<0.001$ & $\mathrm{p}<0.001$ & $\begin{array}{c}\mathrm{p}< \\
0.001\end{array}$ \\
\hline Sport Level & & $\begin{array}{c}p= \\
0.06\end{array}$ & $\begin{array}{c}\mathrm{p}< \\
0.01\end{array}$ & $\begin{array}{l}\mathrm{p}< \\
0.01\end{array}$ & $\begin{array}{l}\mathrm{p}< \\
0.01\end{array}$ & $\begin{array}{l}\mathrm{p}< \\
0.01\end{array}$ & $\begin{array}{l}\mathrm{p}< \\
0.01\end{array}$ & $\begin{array}{l}\mathrm{p}< \\
0.01\end{array}$ & $\mathrm{p}<0.01$ & $\mathrm{p}<0.01$ & $\mathrm{p}<0.01$ & $\mathrm{p}<0.01$ \\
\hline Freshman & & & $\begin{array}{c}\mathrm{p}< \\
0.0001\end{array}$ & $\begin{array}{c}\mathrm{p}< \\
0.0001\end{array}$ & $\begin{array}{c}\mathrm{p}< \\
0.0001\end{array}$ & $\begin{array}{c}\mathrm{p}< \\
0.0001\end{array}$ & $\begin{array}{c}\mathrm{p}< \\
0.0001\end{array}$ & $\begin{array}{c}\mathrm{p}< \\
0.0001\end{array}$ & $\mathrm{p}<0.0001$ & $\mathrm{p}<0.0001$ & $\begin{array}{c}\mathrm{p}< \\
0.0001\end{array}$ & $\begin{array}{c}\mathrm{p}< \\
0.0001\end{array}$ \\
\hline Previous Concussion & & & & $\begin{array}{c}\mathrm{p}< \\
0.0001\end{array}$ & $\begin{array}{c}\mathrm{p}< \\
0.001\end{array}$ & $\begin{array}{c}\mathrm{p}< \\
0.001\end{array}$ & $\begin{array}{c}\mathrm{p}< \\
0.001\end{array}$ & $\begin{array}{c}\mathrm{p}< \\
0.001\end{array}$ & $\mathrm{p}<0.001$ & $\mathrm{p}<0.001$ & $\mathrm{p}<0.001$ & $\begin{array}{c}\mathrm{p}< \\
0.001\end{array}$ \\
\hline $\begin{array}{l}\text { Headache in the past } \\
\text { three months }\end{array}$ & & & & & $\begin{array}{c}\mathrm{p}< \\
0.001\end{array}$ & $\begin{array}{c}\mathrm{p}< \\
0.001\end{array}$ & $\begin{array}{c}\mathrm{p}< \\
0.001\end{array}$ & $\begin{array}{c}\mathrm{p}< \\
0.001\end{array}$ & $\mathrm{p}<0.01$ & $\mathrm{p}<0.01$ & $\mathrm{p}<0.01$ & $\mathrm{p}<0.01$ \\
\hline Migraine Headache & & & & & & $\begin{array}{c}\mathrm{p}= \\
0.86\end{array}$ & & & & & & \\
\hline ADD/ADHD & & & & & & & $\begin{array}{c}\mathrm{p}< \\
0.001\end{array}$ & $\begin{array}{l}\mathrm{p}< \\
0.01\end{array}$ & $\mathrm{p}<0.001$ & $\mathrm{p}<0.001$ & $\mathrm{p}<0.001$ & $\begin{array}{c}\mathrm{p}< \\
0.001\end{array}$ \\
\hline Depression & & & & & & & & $\begin{array}{c}\mathrm{p}= \\
0.22\end{array}$ & & & & \\
\hline BSI Somatization & & & & & & & & & $\mathrm{p}<0.01$ & $\mathrm{p}=0.02$ & $\mathrm{p}=0.35$ & $\mathrm{p}=0.03$ \\
\hline Sex*Somatization & & & & & & & & & $\mathrm{p}<0.01$ & $\mathrm{p}=0.01$ & $\mathrm{p}=0.02$ & $\mathrm{p}=0.01$ \\
\hline BSI Depression & & & & & & & & & $p=0.29$ & & & \\
\hline BSI Anxiety & & & & & & & & & $\mathrm{p}=0.42$ & & & \\
\hline SCAT Severity Score & & & & & & & & & & & $\mathrm{p}=0.18$ & \\
\hline BSSS & & & & & & & & & & & & $\mathrm{p}=0.77$ \\
\hline -2logLikelihood & 2133.19 & 2127.25 & 1867.21 & 1852.2 & 1838.73 & 1838.7 & 1828.64 & 1827.29 & 1802.9 & 1809.54 & 1800.88 & 1771.62 \\
\hline $\mathrm{BIC}$ & 2136.49 & 2132.74 & 1873.8 & 1859.89 & 1847.52 & 1848.58 & 1838.53 & 1838.28 & 1817.18 & 1821.63 & 1814.07 & 1784.8 \\
\hline
\end{tabular}


Supplementary Table 3.2.2d. Mixed Model Selection - Any Free Time-Related Concussion

\begin{tabular}{|c|c|c|c|c|c|c|c|c|c|c|c|c|}
\hline & $\begin{array}{c}\text { Model } \\
1\end{array}$ & $\begin{array}{c}\text { Model } \\
2\end{array}$ & $\begin{array}{c}\text { Model } \\
3\end{array}$ & $\begin{array}{c}\text { Model } \\
4\end{array}$ & $\begin{array}{c}\text { Model } \\
5\end{array}$ & $\begin{array}{c}\text { Model } \\
6\end{array}$ & $\begin{array}{l}\text { Model } \\
7\end{array}$ & $\begin{array}{c}\text { Model } \\
8\end{array}$ & $\begin{array}{c}\text { Model } \\
9\end{array}$ & Model 10 & Model 11 & Model 12 \\
\hline Sex & $\begin{array}{c}\mathrm{p}< \\
0.0001\end{array}$ & $\begin{array}{c}\mathrm{p}< \\
0.0001\end{array}$ & $\begin{array}{c}\mathrm{p}< \\
0.0001\end{array}$ & $\begin{array}{c}\mathrm{p}< \\
0.0001\end{array}$ & $\begin{array}{c}\mathrm{p}< \\
0.0001\end{array}$ & $\begin{array}{c}\mathrm{p}< \\
0.0001\end{array}$ & $\begin{array}{c}\mathrm{p}< \\
0.0001\end{array}$ & $\begin{array}{c}\mathrm{p}< \\
0.0001\end{array}$ & $\begin{array}{c}\mathrm{p}< \\
0.0001\end{array}$ & $\begin{array}{c}\mathrm{p}< \\
0.0001\end{array}$ & $\begin{array}{c}\mathrm{p}< \\
0.0001\end{array}$ & $\mathrm{p}<0.0001$ \\
\hline Sport Level & & $\mathrm{p}=0.63$ & & & & & & & & & & \\
\hline Freshman & & & $\mathrm{p}=0.04$ & $\mathrm{p}=0.04$ & $\mathrm{p}=0.05$ & $\mathrm{p}=0.04$ & $\mathrm{p}=0.04$ & $\mathrm{p}=0.04$ & $\mathrm{p}=0.05$ & $\mathrm{p}=0.06$ & $\mathrm{p}=0.07$ & \\
\hline $\begin{array}{l}\text { Previous Concussion } \\
\text { Headache in the past three } \\
\text { months }\end{array}$ & & & & $\mathrm{p}=0.01$ & $\begin{array}{l}\mathrm{p}=0.01 \\
\mathrm{p}=0.83\end{array}$ & $\mathrm{p}=0.02$ & $\mathrm{p}=0.01$ & $\mathrm{p}=0.02$ & $\mathrm{p}=0.01$ & $\mathrm{p}=0.01$ & $\mathrm{p}=0.01$ & $\mathrm{p}=0.01$ \\
\hline Migraine Headache & & & & & & $\mathrm{p}=0.33$ & & & & & & \\
\hline ADD/ADHD & & & & & & & $\mathrm{p}=0.52$ & & & & & \\
\hline Depression & & & & & & & & $\mathrm{p}=0.22$ & & & & \\
\hline BSI Somatization & & & & & & & & & $\mathrm{p}=0.67$ & & & \\
\hline BSI Depression & & & & & & & & & $\mathrm{p}=0.40$ & & & \\
\hline BSI Anxiety & & & & & & & & & $\mathrm{p}=0.96$ & & & \\
\hline SCAT Severity Score & & & & & & & & & & $\mathrm{p}=0.89$ & & \\
\hline BSSS & & & & & & & & & & & $\mathrm{p}=0.06$ & $\mathrm{p}=0.06$ \\
\hline -2logLikelihood & 1101.1 & 1100.17 & 1097.21 & 1101.53 & 1091.49 & 1090.69 & 1091.18 & 1090.29 & 1089.81 & 1091.08 & 1067.76 & 1070.87 \\
\hline $\mathrm{BIC}$ & 1104.39 & 1105.66 & 1101.6 & 1097.02 & 1098.08 & 1097.28 & 1097.77 & 1096.88 & 1098.6 & 1097.68 & 1074.35 & 1076.36 \\
\hline
\end{tabular}


Supplementary Table 3.2.2e. Mixed Model Selection - Any Concussion within Varsity Cadets

\begin{tabular}{|c|c|c|c|c|c|c|c|c|c|c|c|c|}
\hline & Model 1 & Model 2 & Model 3 & Model 4 & Model 5 & Model 6 & Model 7 & Model 8 & Model 9 & $\begin{array}{c}\text { Model } \\
10\end{array}$ & $\begin{array}{c}\text { Model } \\
11 \\
\end{array}$ & Model 12 \\
\hline Sex & $\begin{array}{c}\mathrm{p}< \\
0.001\end{array}$ & $\begin{array}{c}\mathrm{p}< \\
0.0001\end{array}$ & $\begin{array}{c}\mathrm{p}< \\
0.0001\end{array}$ & $\begin{array}{c}\mathrm{p}< \\
0.0001\end{array}$ & $\begin{array}{c}\mathrm{p}< \\
0.0001\end{array}$ & $\begin{array}{c}\mathrm{p}< \\
0.0001\end{array}$ & $\begin{array}{c}\mathrm{p}< \\
0.0001\end{array}$ & $\begin{array}{c}\mathrm{p}< \\
0.0001\end{array}$ & $\mathrm{p}<0.01$ & $\mathrm{p}<0.01$ & $\mathrm{p}<0.01$ & $\mathrm{p}<0.001$ \\
\hline Contact Level & & $\begin{array}{c}\mathrm{p}< \\
0.0001\end{array}$ & $\begin{array}{c}\mathrm{p}< \\
0.0001\end{array}$ & $\begin{array}{c}\mathrm{p}< \\
0.001\end{array}$ & $\begin{array}{c}\mathrm{p}< \\
0.001\end{array}$ & $\begin{array}{c}\mathrm{p}< \\
0.001\end{array}$ & $\begin{array}{c}\mathrm{p}< \\
0.001\end{array}$ & $\begin{array}{c}\mathrm{p}< \\
0.001\end{array}$ & $\begin{array}{c}\mathrm{p}< \\
0.0001\end{array}$ & $\begin{array}{c}\mathrm{p}< \\
0.001\end{array}$ & $\begin{array}{c}\mathrm{p}< \\
0.0001\end{array}$ & $\mathrm{p}<0.001$ \\
\hline Freshman & & & $\begin{array}{c}\mathrm{p}< \\
0.0001\end{array}$ & $\begin{array}{c}\mathrm{p}< \\
0.0001\end{array}$ & $\begin{array}{c}\mathrm{p}< \\
0.0001\end{array}$ & $\begin{array}{c}\mathrm{p}< \\
0.0001\end{array}$ & $\begin{array}{c}\mathrm{p}< \\
0.0001\end{array}$ & $\begin{array}{c}\mathrm{p}< \\
0.0001\end{array}$ & $\begin{array}{c}\mathrm{p}< \\
0.0001\end{array}$ & $\begin{array}{c}\mathrm{p}< \\
0.0001\end{array}$ & $\begin{array}{c}\mathrm{p}< \\
0.0001\end{array}$ & $\mathrm{p}<0.0001$ \\
\hline Previous Concussion & & & & $\begin{array}{c}\mathrm{p}< \\
0.0001\end{array}$ & $\begin{array}{c}\mathrm{p}< \\
0.001\end{array}$ & $\begin{array}{c}\mathrm{p}< \\
0.001\end{array}$ & $\begin{array}{c}\mathrm{p}< \\
0.001\end{array}$ & $\begin{array}{c}\mathrm{p}< \\
0.001\end{array}$ & $\begin{array}{c}\mathrm{p}< \\
0.001\end{array}$ & $\begin{array}{c}\mathrm{p}< \\
0.001\end{array}$ & $\begin{array}{c}\mathrm{p}< \\
0.001\end{array}$ & $\mathrm{p}<0.001$ \\
\hline $\begin{array}{l}\text { Headache in the past } \\
\text { three months }\end{array}$ & & & & & $\mathrm{p}<0.01$ & $\mathrm{p}<0.01$ & $\mathrm{p}<0.01$ & $\mathrm{p}<0.01$ & $\mathrm{p}=0.01$ & $\mathrm{p}<0.01$ & $\mathrm{p}<0.01$ & $\mathrm{p}<0.01$ \\
\hline Migraine Headache & & & & & & $\mathrm{p}=0.41$ & & & & & & \\
\hline $\mathrm{ADD} / \mathrm{ADHD}$ & & & & & & & $\mathrm{p}=0.97$ & & & & & \\
\hline Depression & & & & & & & & $\mathrm{p}=0.87$ & & & & \\
\hline BSI Somatization & & & & & & & & & $\mathrm{p}=0.06$ & & & \\
\hline BSI Depression & & & & & & & & & $\mathrm{p}=0.89$ & & & \\
\hline BSI Anxiety & & & & & & & & & $\mathrm{p}=0.42$ & $\mathrm{p}=0.97$ & $\mathrm{p}=0.45$ & $\mathrm{p}=0.90$ \\
\hline Sex*BSI Anxiety & & & & & & & & & $\mathrm{p}=0.02$ & $\mathrm{p}=0.01$ & $\mathrm{p}=0.02$ & $\mathrm{p}=0.01$ \\
\hline SCAT Severity Score & & & & & & & & & & & $\mathrm{p}=0.20$ & \\
\hline BSSS & & & & & & & & & & & & $\mathrm{p}=0.82$ \\
\hline -2logLikelihood & 1566.5 & 1540.45 & 1506.14 & 1490.96 & 1482.23 & 1481.59 & 1482.23 & 1482.2 & 1469.62 & 1473.26 & 1471.65 & 1399.82 \\
\hline $\mathrm{BIC}$ & 1569.8 & 1545.94 & 1512.73 & 1498.65 & 1491.02 & 1491.47 & 1492.11 & 1492.09 & 1482.81 & 1484.24 & 1483.74 & 1411.91 \\
\hline
\end{tabular}


Supplementary Table 3.2.2f. Mixed Model Selection - Any Sport-Related Concussion within Varsity Cadets

\begin{tabular}{|c|c|c|c|c|c|c|c|c|c|c|c|c|c|}
\hline & Model 1 & Model 2 & Model 3 & Model 4 & Model 5 & Model 6 & Model 7 & Model 8 & Model 9 & Model 10 & $\begin{array}{c}\text { Model } \\
11 \\
\end{array}$ & $\begin{array}{c}\text { Model } \\
12 \\
\end{array}$ & $\begin{array}{c}\text { Model } \\
13 \\
\end{array}$ \\
\hline Sex & $\mathrm{p}=0.14$ & $\begin{array}{c}\mathrm{p}<0.01 \\
\mathrm{p}<\end{array}$ & $\begin{array}{c}\mathrm{p}<0.01 \\
\mathrm{p}<\end{array}$ & $\begin{array}{c}\mathrm{p}<0.01 \\
\mathrm{p}<\end{array}$ & $\begin{array}{c}\mathrm{p}<0.01 \\
\mathrm{p}<\end{array}$ & $\begin{array}{c}\mathrm{p}<0.01 \\
\mathrm{p}<\end{array}$ & $\begin{array}{c}\mathrm{p}<0.01 \\
\mathrm{p}<\end{array}$ & $\begin{array}{c}\mathrm{p}<0.01 \\
\mathrm{p}<\end{array}$ & $\begin{array}{c}\mathrm{p}<0.01 \\
\mathrm{p}<\end{array}$ & $\begin{array}{c}\mathrm{p}<0.01 \\
\mathrm{p}<\end{array}$ & $\begin{array}{c}\mathrm{p}<0.01 \\
\mathrm{p}<\end{array}$ & $\begin{array}{c}\mathrm{p}<0.01 \\
\mathrm{p}<\end{array}$ & $\begin{array}{c}\mathrm{p}<0.01 \\
\mathrm{p}<\end{array}$ \\
\hline Contact Level & & 0.0001 & 0.0001 & 0.0001 & 0.0001 & 0.0001 & 0.0001 & 0.0001 & 0.0001 & 0.0001 & 0.0001 & 0.0001 & 0.0001 \\
\hline $\begin{array}{l}\text { Freshman } \\
\text { Previous }\end{array}$ & & & $\mathrm{p}=0.03$ & $\begin{array}{c}\mathrm{p}=0.02 \\
\mathrm{p}<\end{array}$ & $\mathrm{p}=0.04$ & $\mathrm{p}=0.04$ & $\mathrm{p}=0.04$ & $\mathrm{p}=0.04$ & $\mathrm{p}=0.21$ & $\mathrm{p}=0.20$ & & & $\mathrm{p}<$ \\
\hline $\begin{array}{l}\text { Concussion } \\
\text { Headache in the }\end{array}$ & & & & 0.001 & $\mathrm{p}<0.01$ & $\mathrm{p}<0.01$ & $\mathrm{p}<0.01$ & $\mathrm{p}<0.01$ & $\mathrm{p}<0.01$ & $\mathrm{p}<0.01$ & $\mathrm{p}<0.01$ & $\mathrm{p}<0.01$ & 0.001 \\
\hline past three months & & & & & $\mathrm{p}=0.02$ & $\mathrm{p}=0.03$ & $\mathrm{p}=0.02$ & $\mathrm{p}=0.02$ & $\mathrm{p}=0.07$ & $\mathrm{p}=0.07$ & & & \\
\hline Migraine Headache & & & & & & $\mathrm{p}=0.62$ & & & & & & & \\
\hline $\mathrm{ADD} / \mathrm{ADHD}$ & & & & & & & $\mathrm{p}=0.77$ & & & & & & \\
\hline Depression & & & & & & & & $\mathrm{p}=0.98$ & & & & & \\
\hline BSI Somatization & & & & & & & & & $\mathrm{p}=0.09$ & $\mathrm{p}=0.03$ & $\begin{array}{c}\mathrm{p}< \\
0.001\end{array}$ & $\mathrm{p}=0.08$ & $\mathrm{p}<0.01$ \\
\hline BSI Depression & & & & & & & & & $\mathrm{p}=0.65$ & & & & \\
\hline $\begin{array}{l}\text { BSI Anxiety } \\
\text { SCAT Severity }\end{array}$ & & & & & & & & & $\mathrm{p}=0.71$ & & & & \\
\hline Score & & & & & & & & & & & & $\mathrm{p}=0.39$ & \\
\hline BSSS & & & & & & & & & & & & & $\mathrm{p}=0.96$ \\
\hline -2logLikelihood & 1132.46 & 1080.23 & 1075.45 & 1064.72 & 1059.61 & 1059.39 & 1059.53 & 1059.61 & 1054.07 & 1054.33 & 1059.49 & 1058.73 & 1018.78 \\
\hline $\mathrm{BIC}$ & 1135.75 & 1085.72 & 1082.04 & 1072.41 & 1068.4 & 1069.27 & 1069.42 & 1069.5 & 1066.15 & 1064.22 & 1067.18 & 1067.52 & 1027.56 \\
\hline
\end{tabular}


Supplementary Table 3.2.2g. Mixed Model Selection - Any Academy Training-Related Concussion within Varsity Cadets

\begin{tabular}{|c|c|c|c|c|c|c|c|c|c|c|c|}
\hline & Model 1 & Model 2 & Model 3 & Model 4 & Model 5 & Model 6 & Model 7 & Model 8 & Model 9 & Model 10 & Model 11 \\
\hline Sex & $\mathrm{p}=0.06$ & $\mathrm{p}=0.07$ & $\mathrm{p}=0.04$ & $\mathrm{p}=0.03$ & $\mathrm{p}=0.03$ & $\mathrm{p}=0.03$ & $\mathrm{p}=0.03$ & $\mathrm{p}=0.04$ & $\mathrm{p}=0.09$ & $\mathrm{p}=0.03$ & $\mathrm{p}=0.05$ \\
\hline Contact Level & & $\mathrm{p}=0.83$ & $\mathrm{p}<\mathrm{c}$ & 001 & & & & & & & \\
\hline & & & & $\mathrm{p}<$ & $\mathrm{p}<$ & $\mathrm{p}<$ & $\mathrm{p}<$ & $\mathrm{p}<$ & $\mathrm{p}<$ & $\mathrm{p}<$ & $\mathrm{p}<$ \\
\hline Freshman & & & & 0.0001 & 0.0001 & 0.0001 & 0.0001 & 0.0001 & 0.0001 & 0.0001 & 0.0001 \\
\hline Previous Concussion & & & & $\mathrm{p}=0.03$ & $\mathrm{p}=0.03$ & $\mathrm{p}=0.03$ & $\mathrm{p}=0.03$ & $\mathrm{p}=0.03$ & $\mathrm{p}=0.03$ & $\mathrm{p}=0.04$ & $\mathrm{p}=0.06$ \\
\hline $\begin{array}{l}\text { Headache in the past three } \\
\text { months }\end{array}$ & & & & & $p=0.68$ & & & & & & \\
\hline Migraine Headache & & & & & & $\mathrm{p}=0.89$ & & & & & \\
\hline ADD/ADHD & & & & & & & $\mathrm{p}=0.99$ & & & & \\
\hline Depression & & & & & & & & $\mathrm{p}=0.43$ & & & \\
\hline BSI Somatization & & & & & & & & & $\mathrm{p}=0.14$ & & \\
\hline BSI Depression & & & & & & & & & $\mathrm{p}=0.25$ & & \\
\hline BSI Anxiety & & & & & & & & & $\mathrm{p}=0.46$ & & \\
\hline SCAT Severity Score & & & & & & & & & & $\mathrm{p}=0.14$ & \\
\hline BSSS & & & & & & & & & & & $\mathrm{p}=0.89$ \\
\hline -2logLikelihood & 490.58 & 490.22 & 424.64 & 420.35 & 420.18 & 420.33 & 418.48 & 419.83 & 415.4 & 417.48 & 408.59 \\
\hline $\mathrm{BIC}$ & 493.88 & 495.72 & 429.04 & 425.84 & 426.77 & 426.92 & 425.07 & 426.42 & 424.19 & 424.08 & 415.18 \\
\hline
\end{tabular}


Supplementary Table 3.2.2h. Mixed Model Selection - Any Free Time-Related Concussion within Varsity Cadets

\begin{tabular}{|c|c|c|c|c|c|c|c|c|c|c|c|}
\hline & Model 1 & Model 2 & Model 3 & Model 4 & Model 5 & Model 6 & Model 7 & Model 8 & Model 9 & Model 10 & Model 11 \\
\hline Sex & $\mathrm{p}<0.01$ & $\mathrm{p}=0.01$ & $\mathrm{p}<0.01$ & $\mathrm{p}<0.01$ & $\mathrm{p}=0.01$ & $\mathrm{p}<0.01$ & $\mathrm{p}<0.01$ & $\begin{array}{c}\mathrm{p}< \\
0.0001\end{array}$ & $\mathrm{p}<0.001$ & $\mathrm{p}=0.01$ & $\mathrm{p}<0.01$ \\
\hline Contact Level & & $\mathrm{p}=0.85$ & & & & & & & & & \\
\hline Freshman & & & $\mathrm{p}=0.33$ & & & & & & & & \\
\hline Previous Concussion & & & & $\mathrm{p}=0.79$ & & & & & & & \\
\hline Headache in the past three months & & & & & $\mathrm{p}=0.42$ & & & & & & \\
\hline Migraine Headache & & & & & & $\mathrm{p}=0.24$ & & & & & \\
\hline $\mathrm{ADD} / \mathrm{ADHD}$ & & & & & & & $\mathrm{p}=0.99$ & & & & \\
\hline Depression & & & & & & & & $\mathrm{p}=0.99$ & & & \\
\hline BSI Somatization & & & & & & & & & $\mathrm{p}=0.57$ & & \\
\hline BSI Depression & & & & & & & & & $\mathrm{p}=0.47$ & & \\
\hline BSI Anxiety & & & & & & & & & $\mathrm{p}=0.51$ & & \\
\hline SCAT Severity Score & & & & & & & & & & $\mathrm{p}=0.41$ & \\
\hline BSSS & & & & & & & & & & & $\mathrm{p}=0.58$ \\
\hline -2logLikelihood & 295.59 & 295.28 & 294.65 & 295.52 & 294.97 & 294.49 & 294.89 & 295.12 & 294.35 & 294.87 & 292.02 \\
\hline $\mathrm{BIC}$ & 298.88 & 300.77 & 299.04 & 299.91 & 299.37 & 298.89 & 299.28 & 299.52 & 300.94 & 299.26 & 296.41 \\
\hline
\end{tabular}




\section{REFERENCES}

Aare, M., \& Kleiven, S. (2007). Evaluation of head response to ballistic helmet impacts using the finite element method. International Journal of Impact Engineering, 34(3), 596-608.

Abrahams, S., Fie, S. M., Patricios, J., Posthumus, M., \& September, A. V. (2014). Risk factors for sports concussion: an evidence-based systematic review. Br J Sports Med, 48(2), 9197. doi:10.1136/bjsports-2013-092734

American Psychiatric Association. (2013). Diagnostic and Statistical Manual of Mental Disorders: Fifth Edition (DSM-5). Arlington, VA: American Psychiatric Publishing.

Annegers, J. F., Grabow, J. D., Kurland, L. T., \& Laws, E. R., Jr. (1980). The incidence, causes, and secular trends of head trauma in Olmsted County, Minnesota, 1935-1974. Neurology, 30(9), 912-919.

Arfanakis, K., Haughton, V. M., Carew, J. D., Rogers, B. P., Dempsey, R. J., \& Meyerand, M. E. (2002). Diffusion tensor MR imaging in diffuse axonal injury. AJNR Am J Neuroradiol, 23(5), 794-802.

Asken, B. M., DeKosky, S. T., Clugston, J. R., Jaffee, M. S., \& Bauer, R. M. (2017). Diffusion tensor imaging (DTI) findings in adult civilian, military, and sport-related mild traumatic brain injury (mTBI): a systematic critical review. Brain imaging and behavior. doi:10.1007/s11682-017-9708-9

Asken, B. M., McCrea, M. A., Clugston, J. R., Snyder, A. R., Houck, Z. M., \& Bauer, R. M. (2016). "Playing Through It": Delayed Reporting and Removal From Athletic Activity After Concussion Predicts Prolonged Recovery. J Athl Train, 51(4), 329-335. doi:10.4085/1062-6050-51.5.02

Asken, B. M., Snyder, A. R., Clugston, J. R., Gaynor, L. S., Sullan, M. J., \& Bauer, R. M. (2017). Concussion-Like Symptom Reporting in Non-Concussed Collegiate Athletes. Archives of clinical neuropsychology : the official journal of the National Academy of Neuropsychologists, 32(8), 963-971. doi:10.1093/arclin/acx018

Asplund, C. A., McKeag, D. B., \& Olsen, C. H. (2004). Sport-related concussion: factors associated with prolonged return to play. Clin J Sport Med, 14(6), 339-343.

Aubry, M., Cantu, R., Dvorak, J., Graf-Baumann, T., Johnston, K. M., Kelly, J., Lovell, M., McCrory, P., Meeuwisse, W. H., Schamasch, P., \& Grp, C. S. (2002). Summary and agreement statement of the 1st International Symposium on Concussion in Sport, Vienna 2001. Clinical Journal of Sport Medicine, 12(1), 6-11.

Barker-Collo, S., Jones, A., Jones, K., Theadom, A., Dowell, A., Starkey, N., \& Feigin, V. L. (2015). Prevalence, natural course and predictors of depression 1 year following traumatic brain injury from a population-based study in New Zealand. Brain Inj, 29(7-8), 859-865. doi:10.3109/02699052.2015.1004759

Barkhoudarian, G., Hovda, D. A., \& Giza, C. C. (2016). The Molecular Pathophysiology of Concussive Brain Injury - an Update. Phys Med Rehabil Clin N Am, 27(2), 373-393. doi:10.1016/j.pmr.2016.01.003 
Bazarian, J. J., Blyth, B., Mookerjee, S., He, H., \& McDermott, M. P. (2010). Sex differences in outcome after mild traumatic brain injury. J Neurotrauma, 27(3), 527-539. doi:10.1089/neu.2009.1068

Bazarian, J. J., McClung, J., Shah, M. N., Cheng, Y. T., Flesher, W., \& Kraus, J. (2005). Mild traumatic brain injury in the United States, 1998--2000. Brain Inj, 19(2), 85-91.

Bazarian, J. J., Veazie, P., Mookerjee, S., \& Lerner, E. B. (2006). Accuracy of mild traumatic brain injury case ascertainment using ICD-9 codes. Acad Emerg Med, 13(1), 31-38. doi:10.1197/j.aem.2005.07.038

Beckwith, J. G., Greenwald, R. M., Chu, J. J., Crisco, J. J., Rowson, S., Duma, S. M., Broglio, S. P., McAllister, T. W., Guskiewicz, K. M., Mihalik, J. P., Anderson, S., Schnebel, B., Brolinson, P. G., \& Collins, M. W. (2013). Timing of Concussion Diagnosis Is Related to Head Impact Exposure Prior to Injury. Medicine \& Science in Sports \& Exercise, 45(4), 747-754. doi:papers3://publication/doi/10.1249/MSS.0b013e3182793067

Beilock, S. L., \& Carr, T. H. (2001). On the fragility of skilled performance: what governs choking under pressure? J Exp Psychol Gen, 130(4), 701-725.

Bethune, A., da Costa, L., van Niftrik, C. H. B., \& Feinstein, A. (2017). Suicidal Ideation After Mild Traumatic Brain Injury: A Consecutive Canadian Sample. Arch Suicide Res, 21(3), 392-402. doi:10.1080/13811118.2016.1199990

Bleiberg, J., Cernich, A. N., Cameron, K., Sun, W., Peck, K., Ecklund, L. T. C. P. J., Reeves, C. D. R. D., Uhorchak, C. O. L. J., Sparling, M. B., Warden, D. L., Bleiberg, J., Ecklund, L. T. C. P. J., Reeves, C. D. R. D., Cernich, A. N., Warden, D. L., Sparling, M. B., Peck, K., Cameron, K., Uhorchak, C. O. L. J., \& Sun, W. (2004). Duration of Cognitive Impairment After Sports Concussion. Neurosurgery, 54, 1073. doi:papers3://publication/doi/10.1227/01.NEU.0000118820.33396.6A

Brainard, L. L., Beckwith, J. G., Chu, J. J., Crisco, J. J., McAllister, T. W., Duhaime, A.-C., Maerlender, A. C., \& Greenwald, R. M. (2012). Gender Differences in Head Impacts Sustained by Collegiate Ice Hockey Players. Medicine \& Science in Sports \& Exercise, 44(2), 297-304. doi:papers3://publication/doi/10.1249/MSS.0b013e31822b0ab4

Breiman, L., Friedman, J., Stone, C., \& Olshen, R. (1984). Classification and regression trees (Wadsworth statistics/probability). Boca Raton: Chapman \& Hall.

Broglio, S. P., Cantu, R. C., Gioia, G. A., Guskiewicz, K. M., Kutcher, J., Palm, M., \& Valovich McLeod, T. C. (2014). National Athletic Trainers' Association position statement: management of sport concussion Journal of Athletic Training (Vol. 49, pp. 245-265): National Athletic Trainers Association.

Broglio, S. P., \& Guskiewicz, K. M. (2014). Management of Collegiate Sport-Related Concussions. In S. M. Slobounov \& W. J. Sebastianelli (Eds.), Concussions in Athletics: From Brain to Behavior. New York: Springer Science + Buisness Media.

Broglio, S. P., Lapointe, A., O'Connor, K. L., \& McCrea, M. (2017). Head Impact Density: A Model to Explain the Elusive Concussion Threshold. J Neurotrauma. doi:10.1089/neu.2016.4767

Broglio, S. P., Macciocchi, S. N., \& Ferrara, M. S. (2007). Neurocognitive performance of concussed athletes when symptom free. J Athl Train, 42(4), 504-508.

Broglio, S. P., McCrea, M., McAllister, T., Harezlak, J., Katz, B., Hack, D., Hainline, B., \& Investigators, C. C. (2017). A National Study on the Effects of Concussion in Collegiate Athletes and US Military Service Academy Members: The NCAA-DoD Concussion 
Assessment, Research and Education (CARE) Consortium Structure and Methods. Sports Med, 47(7), 1437-1451. doi:10.1007/s40279-017-0707-1

Cameron, K. L., Marshall, S. W., Sturdivant, R. X., Lincoln, A. E., Cameron, K. L., Lincoln, A. E., Marshall, S. W., \& Sturdivant, R. X. (2012). Trends in the Incidence of PhysicianDiagnosed Mild Traumatic Brain Injury among Active Duty U.S. Military Personnel between 1997 and 2007. Journal of neurotrauma, 29(7), 1313-1321.

doi:papers3://publication/doi/10.1089/neu.2011.2168

Cancelliere, C., Coronado, V. G., Taylor, C. A., \& Xu, L. (2017). Epidemiology of Isolated Versus Nonisolated Mild Traumatic Brain Injury Treated in Emergency Departments in the United States, 2006-2012: Sociodemographic Characteristics. J Head Trauma Rehabil, 32(4), E37-E46. doi:10.1097/HTR.0000000000000260

Carney, N., Ghajar, J., Jagoda, A., Bedrick, S., Davis-O’Reilly, C., du Coudray, H., Hack, D., Helfand, N., Huddleston, A., Nettleton, T., \& Riggio, S. (2014). Concussion guidelines step 1: systematic review of prevalent indicators. Neurosurgery, 75 Suppl 1, S3-15. doi:papers3://publication/doi/10.1227/NEU.0000000000000433

Carroll, L. J., Cassidy, D., Holm, L., Kraus, J., \& Coronado, V. G. (2004). Methodological Issues and Research Recommendations for Mild Traumatic Brain Injury: The WHO Collaborating Centere Task Force onf Mild Traumatic Brain Injury. Journal of rehabilitation medicine, 43(Suppl), 113-125.

Cassidy, J. D., Carroll, L. J., Peloso, P. M., Borg, J., von Holst, H., Holm, L., Kraus, J., Coronado, V. G., \& Injury, W. H. O. C. C. T. F. o. M. T. B. (2004). Incidence, risk factors and prevention of mild traumatic brain injury: results of the WHO Collaborating Centre Task Force on Mild Traumatic Brain Injury. Journal of rehabilitation medicine(43 Suppl), 28-60.

Centers for Disease Control and Prevention. (2015). Report to Congress on Traumatic Brain Injury in the United States: Epidemiology and Rehabilitation. Retrieved from Atlanta, GA:

Centers for Disease Control and Prevention. (2016a, 1/22/2016). Percent Distributions of TBIrelated Emergency Department Visits by Age Group and Injury Mechanism - United States, 2006-2010. Retrieved from https://www.cdc.gov/traumaticbraininjury/data/dist_ed.html

Centers for Disease Control and Prevention. (2016b, 1/22/2016). Rates of TBI-related Emergency Department Visits by Age Group — United States, 2001-2010. Retrieved from https://www.cdc.gov/traumaticbraininjury/data/rates_ed_byage.html

Centers for Disease Control and Prevention. (2016c, 1/22/2016). Rates of TBI-related Emergency Department Visits, Hospitalizations, and Deaths — United States, 20012010. Retrieved from https://www.cdc.gov/traumaticbraininjury/data/rates.html

Chen, S. H. A., Kareken, D. A., Fastenau, P. S., Trexler, L. E., \& Hutchins, G. D. (2003). A study of persistent post-concussion symptoms in mild head trauma using positron emission tomography. Journal of Neurology Neurosurgery and Psychiatry, 74(3), 326332. doi:DOI 10.1136/jnnp.74.3.326

Chin, E. Y., Nelson, L. D., Barr, W. B., McCrory, P., McCrea, M. A., Chin, E. Y., Nelson, L. D., McCrory, P., McCrea, M. A., \& Barr, W. B. (2016). Reliability and Validity of the Sport Concussion Assessment Tool-3 (SCAT3) in High School and Collegiate Athletes. The American Journal of Sports Medicine. doi:papers3://publication/doi/10.1177/0363546516648141 
Chinn, S. (2000). A simple method for converting an odds ratio to effect size for use in metaanalysis. Statistics in Medicine, 19(22), 3127.

doi:papers3://publication/doi/10.1002/1097-0258(20001130)19:22

Churchill, N. W., Hutchison, M. G., Richards, D., Leung, G., Graham, S. J., \& Schweizer, T. A. (2017). The first week after concussion: Blood flow, brain function and white matter microstructure. Neuroimage Clin, 14, 480-489. doi:10.1016/j.nicl.2017.02.015

Cohen, J. (1988). Statistical power analysis for the behavioral sciences (2nd ed.). Hillsdale, NJ: Lawerence Earlbaum Associates.

Collins, C. L., Fletcher, E. N., Fields, S. K., Kluchurosky, L., Rohrkemper, M. K., Comstock, R. D., \& Cantu, R. C. (2014). Neck Strength: A Protective Factor Reducing Risk for Concussion in High School Sports. Journal of Primary Prevention, 35(5), 309-319. doi:10.1007/s10935-014-0355-2

Covassin, T., Elbin, R. J., Bleecker, A., Lipchik, A., \& Kontos, A. P. (2013). Are there differences in neurocognitive function and symptoms between male and female soccer players after concussions? Am J Sports Med, 41(12), 2890-2895. doi:10.1177/0363546513509962

Covassin, T., Elbin, R. J., Harris, W., Parker, T., \& Kontos, A. (2012). The role of age and sex in symptoms, neurocognitive performance, and postural stability in athletes after concussion. Am J Sports Med, 40(6), 1303-1312. doi:10.1177/0363546512444554

Covassin, T., Moran, R., \& Elbin, R. J. (2016). Sex Differences in Reported Concussion Injury Rates and Time Loss From Participation: An Update of the National Collegiate Athletic Association Injury Surveillance Program From 2004-2005 Through 2008-2009. J Athl Train, 51(3), 189-194. doi:10.4085/1062-6050-51.3.05

Covassin, T., Swanik, C. B., Sachs, M., Kendrick, Z., Schatz, P., Zillmer, E., \& Kaminaris, C. (2006). Sex differences in baseline neuropsychological function and concussion symptoms of collegiate athletes. British Journal of Sports Medicine, 40(11), 923-927discussion 927. doi:papers3://publication/doi/10.1136/bjsm.2006.029496

Covassin, T., Swanik, C. B., \& Sachs, M. L. (2003). Sex differences and the incidence of concussions among collegiate athletes. Journal of Athletic Training.

D'Lauro, C., Johnson, B., Foster, C., McGinty, G., \& Campbell, D. (2017). Costs and contexts: factors affecting self-report of concussion in a military academy. British Journal of Sports Medicine, 51(11), A64.

Daneshvar, D. H., Nowinski, C. J., McKee, A. C., \& Cantu, R. C. (2011). The epidemiology of sport-related concussion. Clinics in sports medicine, 30(1), 1-17, vii. doi:10.1016/j.csm.2010.08.006

Davis-Hayes, C., Gossett, J. D., Levine, W. N., Shams, T., Harada, J., Mitnick, J., \& Noble, J. (2017). Sex-specific Outcomes and Predictors of Concussion Recovery. J Am Acad Orthop Surg, 25(12), 818-828. doi:10.5435/JAAOS-D-17-00276

Decuypere, M., \& Klimo, P., Jr. (2012). Spectrum of traumatic brain injury from mild to severe. Surg Clin North Am, 92(4), 939-957, ix. doi:10.1016/j.suc.2012.04.005

Defense and Veterans Brain Injury Center. (2017, 7/10/2017). DoD Worldwide Numbers for TBI. Retrieved from http://dvbic.dcoe.mil/dod-worldwide-numbers-tbi

DeFord, S. M., Wilson, M. S., Rice, A. C., Clausen, T., Rice, L. K., Barabnova, A., Bullock, R., \& Hamm, R. J. (2002). Repeated mild brain injuries result in cognitive impairment in B6C3F1 mice. J Neurotrauma, 19(4), 427-438. doi:10.1089/08977150252932389 
Delaney, J. S., Abuzeyad, F., Correa, J. A., \& Foxford, R. (2005). Recognition and characteristics of concussions in the emergency department population. J Emerg Med, 29(2), 189-197. doi:10.1016/j.jemermed.2005.01.020

Department of Defense Instruction. (2010). Medical Standards for Appointment, Enlistment, or Induction in the Military Services. (6130.03). Retrieved from http://www.dtic.mil/whs/directives/corres/pdf/613003p.pdf.

Derogatis, L. (2001). BSI 18: Brief Symptom Inventory 18: Administration, Scoring, and Procedure Manual. Minneapolis, MN: NCS Pearson.

Dick, R. W. (2009). Is there a gender difference in concussion incidence and outcomes? British Journal of Sports Medicine, 43 Suppl 1(Suppl_1), i46-50. doi:papers3://publication/doi/10.1136/bjsm.2009.058172

Didehbani, N., Munro Cullum, C., Mansinghani, S., Conover, H., \& Hart, J., Jr. (2013). Depressive symptoms and concussions in aging retired NFL players. Archives of clinical neuropsychology : the official journal of the National Academy of Neuropsychologists, 28(5), 418-424. doi:10.1093/arclin/act028

Doolan, A. W., Day, D. D., Maerlender, A. C., Goforth, M., \& Gunnar Brolinson, P. (2012). A review of return to play issues and sports-related concussion. Ann Biomed Eng, 40(1), 106-113. doi:10.1007/s10439-011-0413-3

Doumas, M., Smolders, C., Brunfaut, E., Bouckaert, F., \& Krampe, R. T. (2012). Dual task performance of working memory and postural control in major depressive disorder. Neuropsychology, 26(1), 110-118. doi:10.1037/a0026181

Duhaime, A.-C., Beckwith, J. G., Maerlender, A. C., McAllister, T. W., Crisco, J. J., Duma, S. M., Brolinson, P. G., Rowson, S., Flashman, L. A., Chu, J. J., \& Greenwald, R. M. (2012). Spectrum of acute clinical characteristics of diagnosed concussions in college athletes wearing instrumented helmets. Journal of Neurosurgery, 117(6), 1092-1099. doi:papers3://publication/doi/10.3171/2012.8.JNS112298

Echemendia, R. J., \& Cantu, R. C. (2003). Return to play following sports-related mild traumatic brain injury: the role for neuropsychology. Appl Neuropsychol, 10(1), 48-55. doi:10.1207/S15324826AN1001_7

Eckner, J. T., Oh, Y. K., Joshi, M. S., Richardson, J. K., \& Ashton-Miller, J. A. (2014). Effect of neck muscle strength and anticipatory cervical muscle activation on the kinematic response of the head to impulsive loads. Am J Sports Med, 42(3), 566-576. doi: $10.1177 / 0363546513517869$

Eierud, C., Craddock, R. C., Fletcher, S., Aulakh, M., King-Casas, B., Kuehl, D., \& LaConte, S. M. (2014). Neuroimaging after mild traumatic brain injury: review and meta-analysis. Neuroimage Clin, 4, 283-294. doi:10.1016/j.nicl.2013.12.009

Eisenberg, M. A., Andrea, J., Meehan, W., \& Mannix, R. (2013). Time interval between concussions and symptom duration. Pediatrics, 132(1), 8-17. doi:10.1542/peds.20130432

Elbin, R. J., Sufrinko, A., Schatz, P., French, J., Henry, L., Burkhart, S., Collins, M. W., \& Kontos, A. P. (2016). Removal From Play After Concussion and Recovery Time. Pediatrics, 138(3). doi:10.1542/peds.2016-0910

Erlanger, D., Kaushik, T., Cantu, R., Barth, J. T., Broshek, D. K., Freeman, J. R., \& Webbe, F. M. (2003). Symptom-based assessment of the severity of a concussion. J Neurosurg, 98(3), 477-484. doi:10.3171/jns.2003.98.3.0477 
Eysenck, M. W., Derakshan, N., Santos, R., \& Calvo, M. G. (2007). Anxiety and cognitive performance: attentional control theory. Emotion, 7(2), 336-353. doi:10.1037/15283542.7.2.336

Farkas, O., Lifshitz, J., \& Povlishock, J. T. (2006). Mechanoporation induced by diffuse traumatic brain injury: an irreversible or reversible response to injury? $J$ Neurosci, 26(12), 3130-3140. doi:10.1523/JNEUROSCI.5119-05.2006

Feng, Y., Abney, T. M., Okamoto, R. J., Pless, R. B., Genin, G. M., \& Bayly, P. V. (2010). Relative brain displacement and deformation during constrained mild frontal head impact. J R Soc Interface, 7(53), 1677-1688. doi:10.1098/rsif.2010.0210

Fife, D. (1987). Head injury with and without hospital admission: comparisons of incidence and short-term disability. American journal of public health, 77(7), 810-812.

Floris, R., Vagnozzi, R., Tarascio, G., Amorini, A. M., Delfini, R., Marziali, S., Lazzarino, G., Signoretti, S., Di Pietro, V., Ludovici, A., \& Tavazzi, B. (2008). Temporal window of metabolic brain vulnerability to concussion: a pilot $1 \mathrm{H}$-magnetic resonance spectroscopic study in concussed athletes--part III. Neurosurgery, 62(6), 1286-1295- discussion 12951286. doi:papers3://publication/doi/10.1227/01.neu.0000333300.34189.74

Franke, L. M., Czarnota, J. N., Ketchum, J. M., \& Walker, W. C. (2015). Factor analysis of persistent postconcussive symptoms within a military sample with blast exposure. $J$ Head Trauma Rehabil, 30(1), E34-46. doi:10.1097/HTR.0000000000000042

Fu, T. S., Jing, R., Fu, W. W., \& Cusimano, M. D. (2016). Epidemiological Trends of Traumatic Brain Injury Identified in the Emergency Department in a Publicly-Insured Population, 2002-2010. PloS one, 11(1), e0145469. doi:10.1371/journal.pone.0145469

Funk, J. R., Rowson, S., Daniel, R. W., \& Duma, S. M. (2011). Validation of Concussion Risk Curves for Collegiate Football Players Derived from HITS Data. Annals of Biomedical Engineering, 40(1), 79-89. doi:papers3://publication/doi/10.1007/s10439-011-0400-8

Gardner, A., Iverson, G. L., \& Stanwell, P. (2014). A systematic review of proton magnetic resonance spectroscopy findings in sport-related concussion. J Neurotrauma, 31(1), 1-18. doi:10.1089/neu.2013.3079

Gaw, C. E., \& Zonfrillo, M. R. (2016). Emergency department visits for head trauma in the United States. BMC Emerg Med, 16, 5. doi:10.1186/s12873-016-0071-8

Gessel, L. M., Fields, S. K., Collins, C. L., Dick, R. W., \& Comstock, R. D. (2007). Concussions among united states high school and collegiate athletes. Journal of Athletic Training, 42(4), 495-503.

Giza, C. C., \& Hovda, D. A. (2014). The new neurometabolic cascade of concussion. Neurosurgery, 75 Suppl 4, S24-33. doi:10.1227/NEU.0000000000000505

Gur, R. C., Gur, R. E., Resnick, S. M., Skolnick, B. E., Alavi, A., \& Reivich, M. (1987). The effect of anxiety on cortical cerebral blood flow and metabolism. J Cereb Blood Flow Metab, 7(2), 173-177. doi:10.1038/jcbfm.1987.40

Guskiewicz, K. M., Marshall, S. W., Bailes, J., McCrea, M., Cantu, R. C., Randolph, C., \& Jordan, B. D. (2005). Association between Recurrent Concussion and Late-Life Cognitive Impairment in Retired Professional Football Players. Neurosurgery, 57(4), 719-726. doi:papers3://publication/doi/10.1227/01.NEU.0000175725.75780.DD

Guskiewicz, K. M., Marshall, S. W., Bailes, J., McCrea, M., Harding, H. P., Jr., Matthews, A., Mihalik, J. R., \& Cantu, R. C. (2007). Recurrent concussion and risk of depression in retired professional football players. Medicine \& Science in Sports \& Exercise, 39(6), 903-909. doi:papers3://publication/doi/10.1249/mss.0b013e3180383da5 
Guskiewicz, K. M., McCrea, M., Marshall, S. W., Cantu, R. C., Randolph, C., Barr, W., Onate, J. A., \& Kelly, J. P. (2003). Cumulative effects associated with recurrent concussion in collegiate football players - The NCAA Concussion Study. JAMA, 290(19), 2549-2555. doi:papers3://publication/doi/10.1001/jama.290.19.2549

Guskiewicz, K. M., \& Mihalik, J. P. (2011). Biomechanics of Sport Concussion: Quest for the Elusive Injury Threshold. Exercise and Sport Sciences Reviews, 39(1), 4-11. doi:papers3://publication/doi/10.1097/JES.0b013e318201f53e

Guskiewicz, K. M., Mihalik, J. P., Shankar, V., Crowell, D. H., Oliaro, S. M., \& Hooker, D. N. (2007). Measurement Of Head Impacts In Collegiate Football Players: Relationship Between Head Impact Biomechanics And Acute Clinical Outcome After Concussion. Neurosurgery, 61(6), 1244-1253. doi:papers3://publication/doi/10.1227/01.NEU.0000280146.37163.79

Guskiewicz, K. M., Ross, S. E., Ross, S. E., \& Guskiewicz, K. M. (2001). Postural stability and neuropsychological deficits after concussion in collegiate athletes. Journal of Athletic Training.

Hainline, B. (2016). Concussion and the NCAA: Report from the Chief Medical Officer. The Bridge, 46(1), 39-44.

Hanninen, T., Tuominen, M., Parkkari, J., Vartiainen, M., Ohman, J., Iverson, G. L., \& Luoto, T. M. (2016). Sport concussion assessment tool - 3rd edition - normative reference values for professional ice hockey players. J Sci Med Sport, 19(8), 636-641. doi:10.1016/j.jsams.2015.08.005

Harmon, K. G., Drezner, J. A., Gammons, M., Guskiewicz, K. M., Halstead, M., Herring, S. A., Kutcher, J. S., Pana, A., Putukian, M., \& Roberts, W. O. (2013). American Medical Society for Sports Medicine position statement: concussion in sport. Br J Sports Med, 47(1), 15-26. doi:10.1136/bjsports-2012-091941

Harris, J. D., Abrams, G. D., Bach, B. R., Williams, D., Heidloff, D., Bush-Joseph, C. A., Verma, N. N., Forsythe, B., \& Cole, B. J. (2014). Return to sport after ACL reconstruction. Orthopedics, 37(2), e103-108. doi:10.3928/01477447-20140124-10

Helmick, K. M., Spells, C. A., Malik, S. Z., Davies, C. A., Marion, D. W., \& Hinds, S. R. (2015). Traumatic brain injury in the US military: epidemiology and key clinical and research programs. Brain imaging and behavior, 9(3), 358-366. doi:10.1007/s11682-0159399-z

Henningsen, P., Zimmermann, T., \& Sattel, H. (2003). Medically unexplained physical symptoms, anxiety, and depression: a meta-analytic review. Psychosom Med, 65(4), 528533.

Henry, L. C., Elbin, R. J., Collins, M. W., Marchetti, G., \& Kontos, A. P. (2016). Examining Recovery Trajectories After Sport-Related Concussion With a Multimodal Clinical Assessment Approach. Neurosurgery, 78(2), 232-241. doi:10.1227/NEU.0000000000001041

Henry, L. C., Tremblay, S., Boulanger, Y., Ellemberg, D., \& Lassonde, M. (2010). Neurometabolic changes in the acute phase after sports concussions correlate with symptom severity. J Neurotrauma, 27(1), 65-76. doi:10.1089/neu.2009.0962

Henry, L. C., Tremblay, S., Leclerc, S., Khiat, A., Boulanger, Y., Ellemberg, D., \& Lassonde, M. (2011). Metabolic changes in concussed American football players during the acute and chronic post-injury phases. BMC Neurol, 11, 105. doi:10.1186/1471-2377-11-105 
Heyer, G. L., Schaffer, C. E., Rose, S. C., Young, J. A., McNally, K. A., \& Fischer, A. N. (2016). Specific Factors Influence Postconcussion Symptom Duration among Youth Referred to a Sports Concussion Clinic. J Pediatr, 174, 33-38 e32. doi:10.1016/j.jpeds.2016.03.014

Hoge, C. W., McGurk, D., Thomas, J. L., Cox, A. L., Engel, C. C., \& Castro, C. A. (2008). Mild traumatic brain injury in U.S. Soldiers returning from Iraq. New England Journal of Medicine, 358(5), 453-463. doi:10.1056/NEJMoa072972

Holbourn, A. H. S. (1943). Mechanics of head injuries. The Lancet, ii, 438-441.

Hootman, J. M., Agel, J., \& Dick, R. (2007). Epidemiology of collegiate injuries for 15 sports: summary and recommendations for injury prevention initiatives. Journal of Athletic Training.

Hoyle, R. H., Stephenson, M. T., Palmgreen, P., Lorch, E. P., \& Donohew, R. L. (2002). Reliability and validity of a brief measure of sensation seeking. Personality and Individual Differences, 32(3), 401-414. doi:Doi 10.1016/S0191-8869(01)00032-0

Hutchison, M., Mainwaring, L. M., Comper, P., Richards, D. W., \& Bisschop, S. M. (2009). Differential emotional responses of varsity athletes to concussion and musculoskeletal injuries. Clin J Sport Med, 19(1), 13-19. doi:10.1097/JSM.0b013e318190ba06

Iacobucci, D. (2012). Mediation analysis and categorical variables: The final frontier. Journal of Consumer Psychology, 22(4), 582-594. doi:10.1016/j.jcps.2012.03.006

Iverson, G. (2007). Predicting slow recovery from sport-related concussion: the new simplecomplex distinction. Clin J Sport Med, 17(1), 31-37.

doi:10.1097/JSM.0b013e3180305e4d

Iverson, G. L., Gaetz, M., Lovell, M. R., Collins, M. W., Gaetz, M., Iverson, G. L., Lovell, M. R., \& Collins, M. W. (2009). Cumulative effects of concussion in amateur athletes. Brain Injury. doi:papers3://publication/doi/10.1080/02699050310001617352

Iverson, G. L., Gardner, A. J., Terry, D. P., Ponsford, J. L., Sills, A. K., Broshek, D. K., \& Solomon, G. S. (2017). Predictors of clinical recovery from concussion: a systematic review. Br J Sports Med, 51(12), 941-948. doi:10.1136/bjsports-2017-097729

Iverson, G. L., Langlois, J. A., McCrea, M. A., \& Kelly, J. P. (2009). Challenges associated with post-deployment screening for mild traumatic brain injury in military personnel. Clin Neuropsychol, 23(8), 1299-1314. doi:10.1080/13854040903153902

Iverson, G. L., Lovell, M. R., Collins, M. W., Collins, M. W., Iverson, G. L., \& Lovell, M. R. (2010). Interpreting Change on ImPACT Following Sport Concussion. The Clinical Neuropsychologist. doi:papers3://publication/doi/10.1076/nten20.v017.i04;page:string:Article/Chapter

Iverson, G. L., Wojtowicz, M., Brooks, B. L., Maxwell, B. A., Atkins, J. E., Zafonte, R., \& Berkner, P. D. (2016). High School Athletes With ADHD and Learning Difficulties Have a Greater Lifetime Concussion History. J Atten Disord. doi:10.1177/1087054716657410

Ivins, B. J., \& Ivins, B. J. (2010). Hospitalization associated with traumatic brain injury in the active duty US Army: 2000-2006. NeuroRehabilitation, 26, 199-212. doi:papers3://publication/doi/10.3233/NRE-2010-0556

Jantzen, K. J., Anderson, B., Steinberg, F. L., \& Kelso, J. A. (2004). A prospective functional MR imaging study of mild traumatic brain injury in college football players. AJNR Am J Neuroradiol, 25(5), 738-745.

Johnson, B., Zhang, K., Gay, M., Horovitz, S., Hallett, M., Sebastianelli, W., \& Slobounov, S. (2012). Alteration of brain default network in subacute phase of injury in concussed 
individuals: resting-state fMRI study. NeuroImage, 59(1), 511-518.

doi:10.1016/j.neuroimage.2011.07.081

Johnson, G. (2014). Athletics participation rates continue to rise. Retrieved from Indianapolis, IN: http://www.ncaa.org/about/resources/media-center/news/athletics-participation-ratescontinue-rise

Jones, G. (2000). Posture. In E. Kandel, J. Schwartz, \& T. Jessell (Eds.), Princicples of Neural Science (4th ed., pp. 816-830): McGraw-Hill.

Junge, A. (2000). The influence of psychological factors on sports injuries. Review of the literature. Am J Sports Med, 28(5 Suppl), S10-15.

Kapfhammer, H. P. (2006). Somatic symptoms in depression. Dialogues Clin Neurosci, 8(2), 227-239.

Katayama, Y., Becker, D. P., Tamura, T., \& Hovda, D. A. (1990). Massive increases in extracellular potassium and the indiscriminate release of glutamate following concussive brain injury. J Neurosurg, 73(6), 889-900. doi:10.3171/jns.1990.73.6.0889

Kawamata, T., Katayama, Y., Hovda, D. A., Yoshino, A., \& Becker, D. P. (1992). Administration of Excitatory Amino-Acid Antagonists Via Microdialysis Attenuates the Increase in Glucose-Utilization Seen Following Concussive Brain Injury. Journal of Cerebral Blood Flow and Metabolism, 12(1), 12-24.

Kawamata, T., Katayama, Y., Hovda, D. A., Yoshino, A., \& Becker, D. P. (1995). Lactate accumulation following concussive brain injury: the role of ionic fluxes induced by excitatory amino acids. Brain Res, 674(2), 196-204.

Kelly, J. P., \& Rosenberg, J. H. (1997). Diagnosis and management of concussion in sports. Neurology, 48(3), 575-580.

Kennedy, C. H., Porter Evans, J., Chee, S., Moore, J. L., Barth, J. T., \& Stuessi, K. A. (2012). Return to combat duty after concussive blast injury. Archives of clinical neuropsychology : the official journal of the National Academy of Neuropsychologists, 27(8), 817-827. doi:10.1093/arclin/acs092

Kerr, Z. Y., Evenson, K. R., Rosamond, W. D., Mihalik, J. P., Guskiewicz, K. M., Marshall, S. W., Evenson, K. R., Marshall, S. W., Guskiewicz, K. M., Kerr, Z. Y., Mihalik, J. P., \& Rosamond, W. D. (2014). Association between concussion and mental health in former collegiate athletes. Injury epidemiology, 1, 28. doi:papers3://publication/doi/10.1186/s40621-014-0028-X

Kerr, Z. Y., Marshall, S. W., Harding, H. P., \& Guskiewicz, K. M. (2012). Nine-year risk of depression diagnosis increases with increasing self-reported concussions in retired professional football players. The American Journal of Sports Medicine, 40(10), 22062212. doi:papers3://publication/doi/10.1177/0363546512456193

Kerr, Z. Y., Mihalik, J. P., Guskiewicz, K. M., Rosamond, W. D., Evenson, K. R., \& Marshall, S. W. (2015). Agreement between athlete-recalled and clinically documented concussion histories in former collegiate athletes. Am J Sports Med, 43(3), 606-613. doi: $10.1177 / 0363546514562180$

Kerr, Z. Y., Register-Mihalik, J. K., Kroshus, E., Baugh, C. M., \& Marshall, S. W. (2016). Motivations Associated With Nondisclosure of Self-Reported Concussions in Former Collegiate Athletes. Am J Sports Med, 44(1), 220-225. doi:10.1177/0363546515612082

Kerr, Z. Y., Roos, K. G., Djoko, A., Dalton, S. L., Broglio, S. P., Marshall, S. W., \& Dompier, T. P. (2017). Epidemiologic Measures for Quantifying the Incidence of Concussion in 
National Collegiate Athletic Association Sports. J Athl Train, 52(3), 167-174. doi:10.4085/1062-6050-51.6.05

Kilcoyne, K. G., Dickens, J. F., Svoboda, S. J., Owens, B. D., Cameron, K. L., Sullivan, R. T., \& Rue, J.-P. (2014). Reported Concussion Rates for Three Division I Football Programs: An Evaluation of the New NCAA Concussion Policy. Sports Health: A Multidisciplinary Approach, 6(5), 402-405. doi:papers3://publication/doi/10.1177/1941738113491545

King, A. I., Yang, K. H., Zhang, L., \& Hardy, W. (2003, 2003/01/01). Is head injury caused by linear or angular acceleration, Lisbon, Portugal.

Kirby, S. N., Thie, H. J., Naftel, S., \& Adelson, M. (2010). Diversity and the Success of Entering Classes at the U.S. Service Academies (RB-9496-OSD). Retrieved from Santa Monica, CA: https://www.rand.org/pubs/research_briefs/RB9496.html

Kleiven, S. (2013). Why most traumatic brain injuries are not caused by linear acceleration but skull fractures are. $1(15), 1-5$. doi:papers3://publication/doi/10.3389/fbioe.2013.00015/abstract

Kline, R. B. (2015). Principles and Practice of Structural Equation Modeling (4th ed.): The Guilford Press.

Kontos, A. P., Covassin, T., Elbin, R. J., \& Parker, T. (2012). Depression and neurocognitive performance after concussion among male and female high school and collegiate athletes. Arch Phys Med Rehabil, 93(10), 1751-1756. doi:10.1016/j.apmr.2012.03.032

Kutcher, J. S., \& Eckner, J. T. (2010). At-risk populations in sports-related concussion. Current Sports Medicine Reports, 9(1), 16-20. doi:10.1249/JSR.0b013e3181caa89d

Kutcher, J. S., \& Giza, C. C. (2014). Sports concussion diagnosis and management. Continuum (Minneap Minn), 20(6 Sports Neurology), 1552-1569. doi:10.1212/01.CON.0000458974.78766.58

Kutcher, J. S., McCrory, P., Davis, G., Ptito, A., Meeuwisse, W. H., \& Broglio, S. P. (2013). What evidence exists for new strategies or technologies in the diagnosis of sports concussion and assessment of recovery? British Journal of Sports Medicine, 47(5), 299303. doi:papers3://publication/doi/10.1136/bjsports-2013-092257

Langlois, J. A., Rutland-Brown, W., \& Wald, M. M. (2006). The Epidemiology and Impact of Traumatic Brain Injury: A Brief Overview. The Journal of head trauma rehabilitation, $21(5), 375$.

LaRoche, A. A., Nelson, L. D., Connelly, P. K., Walter, K. D., \& McCrea, M. A. (2016). SportRelated Concussion Reporting and State Legislative Effects. Clinical Journal of Sport Medicine, 26(1), 33-39. doi:papers3://publication/doi/10.1097/JSM.0000000000000192

Lau, B., Lovell, M. R., Collins, M. W., \& Pardini, J. (2009). Neurocognitive and symptom predictors of recovery in high school athletes. Clin J Sport Med, 19(3), 216-221. doi:10.1097/JSM.0b013e31819d6edb

Lau, B. C., Kontos, A. P., Collins, M. W., Mucha, A., \& Lovell, M. R. (2011). Which on-field signs/symptoms predict protracted recovery from sport-related concussion among high school football players? Am J Sports Med, 39(11), 2311-2318. doi: $10.1177 / 0363546511410655$

Lee, D. J., Meehan, R. T., Robinson, C., Mabry, T. R., \& Smith, M. L. (1992). Immune responsiveness and risk of illness in U.S. Air Force Academy cadets during basic cadet training. Aviat Space Environ Med, 63(6), 517-523.

Lee, E., Desu, M., \& Gehan, E. (1975). Monte Carlo Study of the Power of Some Two-Sample Tests. Biometrika, 62(2), 425-432. 
Li, H., Moreland, J. J., Peek-Asa, C., \& Yang, J. (2017). Preseason Anxiety and Depressive Symptoms and Prospective Injury Risk in Collegiate Athletes. Am J Sports Med, 45(9), 2148-2155. doi:10.1177/0363546517702847

Liao, Y., Huang, X., Wu, Q., Yang, C., Kuang, W., Du, M., Lui, S., Yue, Q., Chan, R. C., Kemp, G. J., \& Gong, Q. (2013). Is depression a disconnection syndrome? Meta-analysis of diffusion tensor imaging studies in patients with MDD. J Psychiatry Neurosci, 38(1), 4956. doi:10.1503/jpn. 110180

Libertiaux, V., Pascon, F., \& Cescotto, S. (2011). Experimental verification of brain tissue incompressibility using digital image correlation. Journal of the Mechanical Behavior of Biomedical Materials, 4(7), 1177-1185. doi:10.1016/j.jmbbm.2011.03.028

Lincoln, A. E., Caswell, S. V., Almquist, J. L., Dunn, R. E., Norris, J. B., \& Hinton, R. Y. (2011). Trends in concussion incidence in high school sports: a prospective 11-year study. Am J Sports Med, 39(5), 958-963. doi:10.1177/0363546510392326

Llewellyn, T., Burdette, G. T., Joyner, A. B., \& Buckley, T. A. (2014). Concussion Reporting Rates at the Conclusion of an Intercollegiate Athletic Career. Clinical Journal of Sport Medicine, 24(1), 76-79. doi:papers3://publication/doi/10.1097/01.jsm.0000432853.77520.3d

Losoi, H., Silverberg, N. D., Waljas, M., Turunen, S., Rosti-Otajarvi, E., Helminen, M., Luoto, T. M., Julkunen, J., Ohman, J., \& Iverson, G. L. (2016). Recovery from Mild Traumatic Brain Injury in Previously Healthy Adults. J Neurotrauma, 33(8), 766-776. doi:10.1089/neu.2015.4070

Lynall, R. C., Mauntel, T. C., Padua, D. A., \& Mihalik, J. P. (2015). Acute Lower Extremity Injury Rates Increase after Concussion in College Athletes. Medicine \& Science in Sports \& Exercise, 47(12), 2487-2492. doi:papers3://publication/doi/10.1249/MSS.0000000000000716

Mac Donald, C. L., Johnson, A. M., Wierzechowski, L., Kassner, E., Stewart, T., Nelson, E. C., Werner, N. J., Adam, O. R., Rivet, D. J., Flaherty, S. F., Oh, J. S., Zonies, D., Fang, R., \& Brody, D. L. (2017). Outcome Trends after US Military Concussive Traumatic Brain Injury. J Neurotrauma, 34(14), 2206-2219. doi:10.1089/neu.2016.4434

Mainwaring, L. M., Hutchison, M., Bisschop, S. M., Comper, P., \& Richards, D. W. (2010). Emotional response to sport concussion compared to ACL injury. Brain Inj, 24(4), 589597. doi:10.3109/02699051003610508

Makdissi, M., Darby, D., Maruff, P., Ugoni, A., Brukner, P., \& McCrory, P. R. (2010). Natural history of concussion in sport: markers of severity and implications for management. Am J Sports Med, 38(3), 464-471. doi:10.1177/0363546509349491

Manley, G., Gardner, A. J., Schneider, K. J., Guskiewicz, K. M., Bailes, J., Cantu, R. C., Castellani, R. J., Turner, M., Jordan, B. D., Randolph, C., Dvorak, J., Hayden, K. A., Tator, C. H., McCrory, P., \& Iverson, G. L. (2017). A systematic review of potential long-term effects of sport-related concussion. Br J Sports Med, 51(12), 969-977. doi:10.1136/bjsports-2017-097791

Marazziti, D., Consoli, G., Picchetti, M., Carlini, M., \& Faravelli, L. (2010). Cognitive impairment in major depression. Eur J Pharmacol, 626(1), 83-86. doi:10.1016/j.ejphar.2009.08.046

Marshall, S. W., Guskiewicz, K. M., Shankar, V., McCrea, M., \& Cantu, R. C. (2015). Epidemiology of sports-related concussion in seven US high school and collegiate sports. Injury epidemiology, 2(1), 13. doi:10.1186/s40621-015-0045-4 
McAllister, T. W., Saykin, A. J., Flashman, L. A., \& Sparling, M. B. (1999). Brain activation during working memory 1 month after mild traumatic brain injury A functional MRI study. Neurology, 53(6), 1300. doi:papers3://publication/doi/10.1212/wnl.53.6.1300

McCrea, M. (2001). Standardized Mental Status Testing on the Sideline after Sport-Related Concussion. Journal of Athletic Training, 36(3), 274. doi:papers3://publication/doi/10.1097/00042752-200107000-00008

McCrea, M., Barr, W., Guskiewicz, K., Randolph, C., Marshall, S. W., Cantu, R., Onate, J., \& Kelly, J. (2005). Standard regression-based methods for measuring recovery after sportrelated concussion. Journal of the International Neuropsychological Society, 11(1), 5869. doi:papers3://publication/doi/10.1017/S1355617705050083

McCrea, M., Guskiewicz, K., Randolph, C., Barr, W. B., Hammeke, T. A., Marshall, S. W., Powell, M. R., Woo Ahn, K., Wang, Y., \& Kelly, J. P. (2013). Incidence, Clinical Course, and Predictors of Prolonged Recovery Time Following Sport-Related Concussion in High School and College Athletes. Journal of the International Neuropsychological Society, 19, 22-33. doi:papers3://publication/doi/10.1017/S1355617712000872

McCrea, M., Guskiewicz, K. M., Marshall, S. W., Barr, W., Randolph, C., Cantu, R. C., Onate, J. A., Yang, J., \& Kelly, J. P. (2003). Acute effects and recovery time following concussion in collegiate football players: the NCAA Concussion Study. JAMA, 290(19), 2556-2563. doi:papers3://publication/doi/10.1001/jama.290.19.2556

McCrea, M., Hammeke, T., Olsen, G., Leo, P., \& Guskiewicz, K. (2004). Unreported Concussion in High School Football Players. Clinical Journal of Sport Medicine, 14(1), 13-17. doi:papers3://publication/doi/10.1097/00042752-200401000-00003

McCrea, M., Kelly, J. P., Randolph, C., Kluge, J., Bartolic, E., Finn, G., Baxter, B., Bartolic, E., Kelly, J. P., Baxter, B., Kluge, J., Finn, G., McCrea, M., \& Randolph, C. (1998). Standardized Assessment of Concussion (SAC): On-Site Mental Status Evaluation of the Athlete. The Journal of head trauma rehabilitation, 13, 27.

McCrory, P. (2001). What's in a name? Br J Sports Med, 35(5), 285-286.

McCrory, P., Meeuwisse, W., Aubry, M., Cantu, B., Dvořák, J., Echemendia, R. J., Engebretsen, L., Johnston, K., Kutcher, J. S., Raftery, M., \& Sills, A. (2013). Consensus Statement on Concussion in Sport - the 4th International Conference on Concussion in Sport Held in Zurich, November 2012. Clinical Journal of Sport Medicine, 23(2), 89-117. doi:papers3://publication/doi/10.1097/JSM.0b013e31828b67cf

McCrory, P., Meeuwisse, W., Dvořák, J., Aubry, M., Bailes, J., Broglio, S., Cantu, R. C., Cassidy, J. D., Echemendia, R. J., Castellani, R. J., Davis, G. A., Ellenbogen, R., Emery, C. A., Engebretsen, L., Feddermann-Demont, N., Giza, C. C., Guskiewicz, K. M., Herring, S. A., Iverson, G. L., Johnston, K. M., Kissick, J., Kutcher, J., Leddy, J. J., Maddocks, D., Makdissi, M., Manley, G., McCrea, M. A., Meehan, W. P., Nagahiro, S., Patricios, J., Putukian, M., Schneider, K., Sills, A., Tator, C. H., Turner, M., Vos, P. E., Pierrot-Deseilligny, C., Milea, D., \& Muri, R. M. (2017). Consensus statement on concussion in sport - the 5th international conference on concussion in sport held in Berlin, October 2016. British Journal of Sports Medicine, 51(11), 838-847. doi:papers3://publication/doi/10.1136/bjsports-2017-097699

McCrory, P., Meeuwisse, W., Johnston, K., Dvorak, J., Aubry, M., Molloy, M., \& Cantu, R. (2009). Consensus Statement on Concussion in Sport: the 3rd International Conference 
on Concussion in Sport held in Zurich, November 2008. Br J Sports Med, 43 Suppl 1, i76-90. doi:10.1136/bjsm.2009.058248

McCrory, P., Meeuwisse, W. H., Aubry, M., Cantu, B., Dvořák, J., Echemendia, R. J., Engebretsen, L., Johnston, K., Kutcher, J. S., Raftery, M., Sills, A., Benson, B. W., Davis, G. A., Ellenbogen, R. G., Guskiewicz, K. M., Herring, S. A., Iverson, G. L., Jordan, B. D., Kissick, J., McCrea, M., McIntosh, A. S., Maddocks, D., Makdissi, M., Purcell, L., Putukian, M., Schneider, K., Tator, C. H., \& Turner, M. (2013). Consensus statement on concussion in sport: the 4th International Conference on Concussion in Sport held in Zurich, November 2012. British Journal of Sports Medicine, 47(5), 250258.

McCuen, E., Svaldi, D., Breedlove, K., Kraz, N., Cummiskey, B., Breedlove, E. L., Traver, J., Desmond, K. F., Hannemann, R. E., Zanath, E., Guerra, A., Leverenz, L., Talavage, T. M., \& Nauman, E. A. (2016). Collegiate women's soccer players suffer greater cumulative head impacts than their high school counterparts. Journal of Biomechanics, 48(13), 1-4. doi:papers3://publication/doi/10.1016/j.jbiomech.2015.08.003

Meachen, S.-J., Hanks, R. A., Millis, S. R., Rapport, L. J., Rapport, L. J., Millis, S. R., Meachen, S.-J., \& Hanks, R. A. (2008). The Reliability and Validity of the Brief Symptom Inventory-18 in Persons With Traumatic Brain Injury. Archives of Physical Medicine and Rehabilitation, 89(5), 958-965. doi:papers3://publication/doi/10.1016/j.apmr.2007.12.028

Meehan, W. P., 3rd, Mannix, R., Monuteaux, M. C., Stein, C. J., \& Bachur, R. G. (2014). Early symptom burden predicts recovery after sport-related concussion. Neurology, 83(24), 2204-2210. doi:10.1212/WNL.0000000000001073

Meehan, W. P., 3rd, Taylor, A. M., Berkner, P., Sandstrom, N. J., Peluso, M. W., Kurtz, M. M., Pascual-Leone, A., \& Mannix, R. (2016). Division III Collision Sports Are Not Associated with Neurobehavioral Quality of Life. J Neurotrauma, 33(2), 254-259. doi:10.1089/neu.2015.3930

Meier, T. B., Bellgowan, P. S., Bergamino, M., Ling, J. M., \& Mayer, A. R. (2016). Thinner Cortex in Collegiate Football Players With, but not Without, a Self-Reported History of Concussion. J Neurotrauma, 33(4), 330-338. doi:10.1089/neu.2015.3919

Merport, A., \& Recklitis, C. J. (2012). Does the Brief Symptom Inventory-18 case rule apply in adult survivors of childhood cancer? Comparison with the Symptom Checklist-90. $J$ Pediatr Psychol, 37(6), 650-659. doi:10.1093/jpepsy/jss050

Meyer, J. S., Kondo, A., Nomura, F., Sakamoto, K., \& Teraura, T. (1970). Cerebral hemodynamics and metabolism following experimental head injury. J Neurosurg, 32(3), 304-319. doi:10.3171/jns.1970.32.3.0304

Miller, J. H., Gill, C., Kuhn, E. N., Rocque, B. G., Menendez, J. Y., O'Neill, J. A., Agee, B. S., Brown, S. T., Crowther, M., Davis, R. D., Ferguson, D., \& Johnston, J. M. (2016). Predictors of delayed recovery following pediatric sports-related concussion: a casecontrol study. J Neurosurg Pediatr, 17(4), 491-496. doi:10.3171/2015.8.PEDS14332

Miller, N. L., Shattuck, L. G., \& Matsangas, P. (2010). Longitudinal study of sleep patterns of United States Military Academy cadets. Sleep, 33(12), 1623-1631.

Miller, S. C., Whitehead, C. R., Otte, C. N., Wells, T. S., Webb, T. S., Gore, R. K., \& Maynard, C. (2015). Risk for broad-spectrum neuropsychiatric disorders after mild traumatic brain injury in a cohort of US Air Force personnel. Occup Environ Med, 72(8), 560-566. doi:10.1136/oemed-2014-102646 
Moffett, J. R., Ross, B., Arun, P., Madhavarao, C. N., \& Namboodiri, A. M. (2007). NAcetylaspartate in the CNS: from neurodiagnostics to neurobiology. Prog Neurobiol, 81(2), 89-131. doi:10.1016/j.pneurobio.2006.12.003

Morgan, J. (2014). Classification and Regression Tree Analysis. Retrieved from https://www.bu.edu/sph/files/2014/05/MorganCART.pdf

Nakano, Y., Baba, H., Maeshima, H., Kitajima, A., Sakai, Y., Baba, K., Suzuki, T., Mimura, M., \& Arai, H. (2008). Executive dysfunction in medicated, remitted state of major depression. J Affect Disord, 111(1), 46-51. doi:10.1016/j.jad.2008.01.027

Narayana, P. A. (2017). White matter changes in patients with mild traumatic brain injury: MRI perspective. Concussion, 35. doi:10.2217/cnc-2016-0028

National Collegiate Athletic Association. (2017). Graduation Success Rate. Retrieved from Indianapolis, IN: http://www.ncaa.org/about/resources/research/graduation-success-rate

Nelson, L. D., Furger, R. E., Ranson, J., Tarima, S., Hammeke, T. A., Randolph, C., Barr, W. B., Guskiewicz, K., Olsen, C. M., Lerner, E. B., \& McCrea, M. A. (2018). Acute Clinical Predictors of Symptom Recovery in Emergency Department Patients with Uncomplicated Mild Traumatic Brain Injury or Non-Traumatic Brain Injuries. J Neurotrauma, 35(2), 249-259. doi:10.1089/neu.2017.4988

Nelson, L. D., Janecek, J. K., \& McCrea, M. A. (2013). Acute clinical recovery from sportrelated concussion. Neuropsychology review, 23(4), 285-299. doi:10.1007/s11065-0139240-7

Nelson, L. D., Tarima, S., LaRoche, A. A., Hammeke, T. A., Barr, W. B., Guskiewicz, K., Randolph, C., McCrea, M. A., Guskiewicz, K., Hammeke, T. A., Tarima, S., McCrea, M. A., Barr, W. B., LaRoche, A. A., Nelson, L. D., Randolph, C., Nelson, L. D., Tarima, S., LaRoche, A. A., \& Hammeke, T. A. (2016). Preinjury somatization symptoms contribute to clinical recovery after sport-related concussion. Neurology, 86, 1856-1863. doi:papers3://publication/doi/10.1212/WNL.0000000000002679

Nordstrom, A., Edin, B. B., Lindstrom, S., \& Nordstrom, P. (2013). Cognitive function and other risk factors for mild traumatic brain injury in young men: nationwide cohort study. $B M J$, 346, f723. doi:10.1136/bmj.f723

O'Brien, M. J., Howell, D. R., Pepin, M. J., \& Meehan, W. P., 3rd. (2017). Sport-Related Concussions: Symptom Recurrence After Return to Exercise. Orthop J Sports Med, 5(10), 2325967117732516. doi:10.1177/2325967117732516

O'Connor, K. L. (In Prep). Concussion Recovery Trajectories among Service Academy Members.

O'Connor, K. L., Allred, C., Cameron, K. L., Campbell, D. E., Houson, M. N., Johnson, B. J., D'Lauro, C., McGinty, G., O'Donnell, P. G., Peck, K., Svoboda, S. J., McCrea, M., McAllister, T. W., \& Broglio, S. P. (2016). The Prevalence of Concussion within the Military Academies: Findings from the Concussion Assessment, Research, and Education (CARE) Consortium. Paper presented at the 5th International Concussion Consensus Meeting, Berlin.

O'Connor, K. L., Allred, D. C., Cameron, K. L., Campbell, D. E., D'Lauro, C., Houston, M. N., Johnson, B. R., Kelly, T. F., McGinty, G., O'Donnell, P. G., Peck, K., Svoboda, S. J., Pasquina, P., McAllister, T. W., McCrea, M., \& Broglio, S. P. (2018). Descriptive analysis of a baseline concussion battery among United States service academy members: Results from the Concussion Assessment, Research and Education (CARE) Consortium. Military medicine. 
O'Connor, K. L., Campbell, D. E., McGinty, G., \& Zupan, M. (In Prep). A preliminary exploration on the impact of aerobic and muscular fitness with mild traumatic brain injury among service academy members at the United States Air Force Academy. Military medicine.

O'Connor, K. L., Rowson, S., Duma, S. M., \& Broglio, S. P. (2017). Head-Impact-Measurement Devices: A Systematic Review. J Athl Train, 52(3), 206-227. doi:10.4085/10626050.52 .2 .05

Ommaya, A. K. (1985). Biomechanics of head injury: experimental aspects. In A. Nahum \& J. W. Melvin (Eds.), The Biomechanics of Traums (pp. 245-270). Norwalk, CT: Appleton \& Lange.

Paelecke-Habermann, Y., Pohl, J., \& Leplow, B. (2005). Attention and executive functions in remitted major depression patients. J Affect Disord, 89(1-3), 125-135. doi:10.1016/j.jad.2005.09.006

Parascandola, M., \& Weed, D. L. (2001). Causation in epidemiology. J Epidemiol Community Health, 55(12), 905-912.

Partheni, M., Constantoyannis, C., Ferrari, R., Nikiforidis, G., Voulgaris, S., \& Papadakis, N. (2000). A prospective cohort study of the outcome of acute whiplash injury in Greece. Clin Exp Rheumatol, 18(1), 67-70.

Paterno, M. V., Schmitt, L. C., Ford, K. R., Rauh, M. J., Myer, G. D., Huang, B., \& Hewett, T. E. (2010). Biomechanical measures during landing and postural stability predict second anterior cruciate ligament injury after anterior cruciate ligament reconstruction and return to sport. Am J Sports Med, 38(10), 1968-1978. doi:10.1177/0363546510376053

Peck, K. Y., Johnston, D. A., Owens, B. D., \& Cameron, K. L. (2013). The incidence of injury among male and female intercollegiate rugby players. Sports Health: A Multidisciplinary Approach, 5(4), 327-333. doi:papers3://publication/doi/10.1177/1941738113487165

Pellman, E. J., Viano, D. C., Tucker, A. M., Casson, I. R., \& Committee on Mild Traumatic Brain Injury, N. F. L. (2003). Concussion in professional football: location and direction of helmet impacts-Part 2. Neurosurgery, 53(6), 1328-1340; discussion 1340-1321. doi:papers3://publication/doi/10.1227/01.NEU.0000093499.20604.21

Pfaller, A. Y., Nelson, L. D., Apps, J. N., Walter, K. D., \& McCrea, M. A. (2016). Frequency and Outcomes of a Symptom-Free Waiting Period After Sport-Related Concussion. Am J Sports Med, 44(11), 2941-2946. doi:10.1177/0363546516651821

Philipps, D. (2015). Concussions in a Required Class: Boxing at Military Academies. New York Times. Retrieved from https://www.nytimes.com/2015/09/30/us/despite-concussionsboxing-is-still-required-for-military-cadets.html

Piland, S. G., Ferrara, M. S., Macciocchi, S. N., Broglio, S. P., \& Gould, T. E. (2010). Investigation of baseline self-report concussion symptom scores. J Athl Train, 45(3), 273278. doi:10.4085/1062-6050-45.3.273

Piland, S. G., Motl, R. W., Ferrara, M. S., \& Peterson, C. L. (2003). Evidence for the Factorial and Construct Validity of a Self-Report Concussion Symptoms Scale. J Athl Train, 38(2), 104-112.

Post, A., \& Hoshizaki, T. B. (2015). Rotational Acceleration, Brain Tissue Strain, and the Relationship to Concussion. Journal of Biomechanical Engineering, 137(3), 030801. doi:papers3://publication/doi/10.1115/1.4028983 
Powell, J. M., Ferraro, J. V., Dikmen, S. S., Temkin, N. R., \& Bell, K. R. (2008). Accuracy of mild traumatic brain injury diagnosis. Arch Phys Med Rehabil, 89(8), 1550-1555. doi:10.1016/j.apmr.2007.12.035

Prins, M., Greco, T., Alexander, D., \& Giza, C. C. (2013). The pathophysiology of traumatic brain injury at a glance. Disease Models \& Mechanisms, 6(6), 1307-1315. doi:10.1242/dmm.011585

Prins, M. L., Alexander, D., Giza, C. C., \& Hovda, D. A. (2013). Repeated mild traumatic brain injury: mechanisms of cerebral vulnerability. J Neurotrauma, 30(1), 30-38. doi:papers3://publication/doi/10.1089/neu.2012.2399

Prins, M. L., Hales, A., Reger, M., Giza, C. C., \& Hovda, D. A. (2010). Repeat Traumatic Brain Injury in the Juvenile Rat Is Associated with Increased Axonal Injury and Cognitive Impairments. Developmental Neuroscience, 32(5-6), 510-518. doi:10.1159/000316800

Putukian, M. (2011). The acute symptoms of sport-related concussion: diagnosis and on-field management. Clinics in sports medicine, 30(1), 49-61, viii. doi:10.1016/j.csm.2010.09.005

Putukian, M. (2016). The psychological response to injury in student athletes: a narrative review with a focus on mental health. British Journal of Sports Medicine, 50(3), 145-148. doi:papers3://publication/doi/10.1136/bjsports-2015-095586

Radloff, L. (1977). The CES-D Scale A Self-Report Depression Scale for Research in the General Population. Applied Psychologial Measurement, 1(3), 385-401.

Register-Mihalik, J. K., Valovich McLeod, T. C., Linnan, L. A., Guskiewicz, K. M., \& Marshall, S. W. (2017). Relationship Between Concussion History and Concussion Knowledge, Attitudes, and Disclosure Behavior in High School Athletes. Clin J Sport Med, 27(3), 321-324. doi:10.1097/JSM.0000000000000349

Resch, J. E., Brown, C. N., Macciocchi, S. N., Cullum, C. M., Blueitt, D., \& Ferrara, M. S. (2015). A Preliminary Formula to Predict Timing of Symptom Resolution for Collegiate Athletes Diagnosed With Sport Concussion. Journal of Athletic Training, 50(12), 12921298. doi:papers3://publication/doi/10.4085/1062-6050-50.12.03

Rice, S. G., American Academy of Pediatrics Council on Sports, M., Fitness, Medicine, A. A. o. P. C. o. S., Fitness, Rice, S. G., American Academy of Pediatrics Council on Sports, M., \& Fitness. (2008). Medical conditions affecting sports participation. Pediatrics, 121, 841848. doi:papers3://publication/doi/10.1542/peds.2008-0080

Roeder, T. (2017). New Cadets Arrive Thursday as Air Force Academy Basic Training Begins. The Gazette. Retrieved from http://www.military.com/daily-news/2017/06/28/newcadets-arrive-thursday-air-force-academy-basic-training.html

Rosene, J. M., Raksnis, B., Silva, B., Woefel, T., Visich, P. S., Dompier, T. P., \& Kerr, Z. Y. (2017). Comparison of Concussion Rates Between NCAA Division I and Division III Men's and Women's Ice Hockey Players. Am J Sports Med, 45(11), 2622-2629. doi:10.1177/0363546517710005

Rosseel, Y. (2012). lavaan: An R Package for Structural Equation Modeling. Journal of Statistical Software, 48(2), 1-36.

Rothman, K. (2012). Epidemiology: An Introduction (2nd ed.). New York: Oxford.

Rowson, S., Brolinson, G., Goforth, M., Dietter, D., \& Duma, S. (2009). Linear and angular head acceleration measurements in collegiate football. Journal of Biomechanical Engineering, 131(6), 061016. doi:papers3://publication/doi/10.1115/1.3130454 
Rowson, S., Duma, S. M., Beckwith, J. G., Chu, J. J., Greenwald, R. M., Crisco, J. J., Brolinson, P. G., Duhaime, A.-C., McAllister, T. W., \& Maerlender, A. C. (2012). Rotational head kinematics in football impacts: an injury risk function for concussion. Annals of Biomedical Engineering, 40(1), 1-13. doi:papers3://publication/doi/10.1007/s10439-0110392-4

Ryu, W. H., Feinstein, A., Colantonio, A., Streiner, D. L., \& Dawson, D. R. (2009). Early identification and incidence of mild TBI in Ontario. Can J Neurol Sci, 36(4), 429-435.

Sabet, A. A., Christoforou, E., Zatlin, B., Genin, G. M., \& Bayly, P. V. (2008). Deformation of the human brain induced by mild angular head acceleration. J Biomech, 41(2), 307-315. doi:10.1016/j.jbiomech.2007.09.016

Sackeim, H. A., Prohovnik, I., Moeller, J. R., Brown, R. P., Apter, S., Prudic, J., Devanand, D. P., \& Mukherjee, S. (1990). Regional cerebral blood flow in mood disorders. I. Comparison of major depressives and normal controls at rest. Arch Gen Psychiatry, 47(1), 60-70.

Schatz, P. (2010). Long-term test-retest reliability of baseline cognitive assessments using ImPACT. The American Journal of Sports Medicine, 38(1), 47-53. doi:papers3://publication/doi/10.1177/0363546509343805

Schneider, K. J., Meeuwisse, W. H., Kang, J., Schneider, G. M., \& Emery, C. A. (2013). Preseason reports of neck pain, dizziness, and headache as risk factors for concussion in male youth ice hockey players. Clin J Sport Med, 23(4), 267-272. doi:10.1097/JSM.0b013e318281f09f

Schneider, K. J., Meeuwisse, W. H., Nettel-Aguirre, A., Barlow, K., Boyd, L., Kang, J., \& Emery, C. A. (2014). Cervicovestibular rehabilitation in sport-related concussion: a randomised controlled trial. Br J Sports Med, 48(17), 1294-1298. doi:10.1136/bjsports2013-093267

Setnik, L., \& Bazarian, J. J. (2007). The characteristics of patients who do not seek medical treatment for traumatic brain injury. Brain Inj, 21(1), 1-9. doi:10.1080/02699050601111419

Shehata, N., Wiley, J. P., Richea, S., Benson, B. W., Duits, L., \& Meeuwisse, W. H. (2009). Sport concussion assessment tool: baseline values for varsity collision sport athletes. $\mathrm{Br} J$ Sports Med, 43(10), 730-734. doi:10.1136/bjsm.2009.059832

Shenton, M. E., Hamoda, H. M., Schneiderman, J. S., Bouix, S., Pasternak, O., Rathi, Y., Vu, M. A., Purohit, M. P., Helmer, K., Koerte, I., Lin, A. P., Westin, C. F., Kikinis, R., Kubicki, M., Stern, R. A., \& Zafonte, R. (2012). A review of magnetic resonance imaging and diffusion tensor imaging findings in mild traumatic brain injury. Brain imaging and behavior, 6(2), 137-192. doi:10.1007/s11682-012-9156-5

Signoretti, S., Marmarou, A., Tavazzi, B., Lazzarino, G., Beaumont, A., \& Vagnozzi, R. (2001). $\mathrm{N}$-Acetylaspartate reduction as a measure of injury severity and mitochondrial dysfunction following diffuse traumatic brain injury. J Neurotrauma, 18(10), 977-991. doi:10.1089/08977150152693683

Smith, M. S. (1990). Psychosomatic symptoms in adolescence. Med Clin North Am, 74(5), 11211134.

Sneyd, M. J., \& Cox, B. (2011). Commentary: decreasing response rates require investigators to quantify and report the impact of selection bias in case-control studies. Int J Epidemiol, 40(5), 1355-1357. doi:10.1093/ije/dyr123 
Sosin, D. M., Sniezek, J. E., \& Thurman, D. J. (1996). Incidence of mild and moderate brain injury in the United States, 1991. Brain Inj, 10(1), 47-54.

Sosnoff, J. J., Broglio, S. P., Shin, S., \& Ferrara, M. S. (2011). Previous mild traumatic brain injury and postural-control dynamics. Journal of Athletic Training, 46(1), 85-91. doi:papers3://publication/doi/10.4085/1062-6050-46.1.85

Spain, A., Daumas, S., Lifshitz, J., Rhodes, J., Andrews, P. J., Horsburgh, K., \& Fowler, J. H. (2010). Mild fluid percussion injury in mice produces evolving selective axonal pathology and cognitive deficits relevant to human brain injury. $J$ Neurotrauma, 27(8), 1429-1438. doi:10.1089/neu.2010.1288

Spielberger, C. (1989). State-Trait Anxiety Inventory: Bibliography (2nd ed.). Palo Alto, CA: Consulting Psychologists Press.

Stein, M. B., Kessler, R. C., Heeringa, S. G., Jain, S., Campbell-Sills, L., Colpe, L. J., Fullerton, C. S., Nock, M. K., Sampson, N. A., Schoenbaum, M., Sun, X., Thomas, M. L., Ursano, R. J., \& Army, S. c. (2015). Prospective longitudinal evaluation of the effect of deployment-acquired traumatic brain injury on posttraumatic stress and related disorders: results from the Army Study to Assess Risk and Resilience in Servicemembers (Army STARRS). Am J Psychiatry, 172(11), 1101-1111. doi:10.1176/appi.ajp.2015.14121572

Stein, M. B., \& McAllister, T. W. (2009). Exploring the convergence of posttraumatic stress disorder and mild traumatic brain injury. Am J Psychiatry, 166(7), 768-776. doi:10.1176/appi.ajp.2009.08101604

Stemper, B. D., \& Pintar, F. A. (2014). Biomechanics of Concussion. Concussion, 28, 14-27. doi:10.1159/000358748

Stephenson, M. T., Hoyle, R. H., Palmgreen, P., \& Slater, M. D. (2003). Brief measures of sensation seeking for screening and large-scale surveys. Drug Alcohol Depend, 72(3), 279-286.

Tanielian, T., Haycox, L. H., Schell, T. L., Marshall, G. N., Burnam, M. A., Eibner, C., Karney, B. R., Meredith, L. S., Ringel, J. S., Vaiana, M. E., Ringel, J. S., Schell, T. L., Haycox, L. H., Meredith, L. S., Eibner, C., Vaiana, M. E., Burnam, M. A., Tanielian, T., Karney, B. R., \& Marshall, G. N. (2008). Invisible Wounds of War. Summary and Recommendations for Addressing Psychological and Cognitive Injuries. Retrieved from http://oai.dtic.mil/oai/oai?verb=getRecord\&metadataPrefix=html\&identifier=ADA48099 $\underline{2}$

Teel, E. F., Marshall, S. W., Shankar, V., McCrea, M., \& Guskiewicz, K. M. (2017). Predicting Recovery Patterns After Sport-Related Concussion. J Athl Train, 52(3), 288-298. doi:10.4085/1062-6050-52.1.12

Terrio, H., Brenner, L. A., Ivins, B. J., Cho, J. M., Helmick, K., Schwab, K., Scally, K., Bretthauer, R., \& Warden, D. (2009). Traumatic brain injury screening: preliminary findings in a US Army Brigade Combat Team. J Head Trauma Rehabil, 24(1), 14-23. doi:10.1097/HTR.0b013e31819581d8

Tierney, R. T., Sitler, M. R., Swanik, C. B., Swanik, K. A., Higgins, M., \& Torg, J. (2005). Gender differences in head-neck segment dynamic stabilization during head acceleration. Medicine and Science in Sports and Exercise, 37(2), 272-279. doi:10.1249/01.Mss.0000152734.475216.Aa

U.S. Department of Education. (2017). The Condition of Education 2017 (NCES 2017-144). Retrieved from https://nces.ed.gov/fastfacts/display.asp?id=40 
United States Military Academy. (2016). Department of Military Instruction Overview Parent's Weekend. West Point, NY.

United States Military Academy. (2017). Candidate Fitness Assessment Instructions. West Point, NY.

Vagnozzi, R., Marmarou, A., Tavazzi, B., Signoretti, S., Di Pierro, D., del Bolgia, F., Amorini, A. M., Fazzina, G., Sherkat, S., \& Lazzarino, G. (1999). Changes of cerebral energy metabolism and lipid peroxidation in rats leading to mitochondrial dysfunction after diffuse brain injury. J Neurotrauma, 16(10), 903-913. doi:10.1089/neu.1999.16.903

Vagnozzi, R., Signoretti, S., Cristofori, L., Alessandrini, F., Floris, R., Isgro, E., Ria, A., Marziali, S., Zoccatelli, G., Tavazzi, B., Del Bolgia, F., Sorge, R., Broglio, S. P., McIntosh, T. K., \& Lazzarino, G. (2010). Assessment of metabolic brain damage and recovery following mild traumatic brain injury: a multicentre, proton magnetic resonance spectroscopic study in concussed patients. Brain, 133(11), 3232-3242. doi:10.1093/brain/awq200

Vagnozzi, R., Signoretti, S., Tavazzi, B., Floris, R., Ludovici, A., Marziali, S., Tarascio, G., Amorini, A. M., Di Pietro, V., Delfini, R., \& Lazzarino, G. (2008). Temporal window of metabolic brain vulnerability to concussion: a pilot $1 \mathrm{H}$-magnetic resonance spectroscopic study in concussed athletes--part III. Neurosurgery, 62(6), 1286-1295; discussion 12951286. doi:10.1227/01.neu.0000333300.34189.74

Vagnozzi, R., Tavazzi, B., Signoretti, S., Amorini, A. M., Belli, A., Cimatti, M., Delfini, R., Di Pietro, V., Finocchiaro, A., \& Lazzarino, G. (2007). Temporal window of metabolic brain vulnerability to concussions: mitochondrial-related impairment--part I. Neurosurgery, 61(2), 379-388; discussion 388-379. doi:10.1227/01.NEU.0000280002.41696.D8

van der Naalt, J., Timmerman, M. E., de Koning, M. E., van der Horn, H. J., Scheenen, M. E., Jacobs, B., Hageman, G., Yilmaz, T., Roks, G., \& Spikman, J. M. (2017). Early predictors of outcome after mild traumatic brain injury (UPFRONT): an observational cohort study. The Lancet. Neurology, 16(7), 532-540. doi:10.1016/S14744422(17)30117-5

Vargas, G., Rabinowitz, A., Meyer, J., \& Arnett, P. A. (2015). Predictors and prevalence of postconcussion depression symptoms in collegiate athletes. J Athl Train, 50(3), 250-255. doi:10.4085/1062-6050-50.3.02

Verweij, B. H., Muizelaar, J. P., Vinas, F. C., Peterson, P. L., Xiong, Y., \& Lee, C. P. (1997). Mitochondrial dysfunction after experimental and human brain injury and its possible reversal with a selective N-type calcium channel antagonist (SNX-111). Neurol Res, 19(3), 334-339.

Voss, J. D., Connolly, J., Schwab, K. A., \& Scher, A. I. (2015). Update on the Epidemiology of Concussion/Mild Traumatic Brain Injury. Current pain and headache reports, 19(7), 32. doi:10.1007/s11916-015-0506-Z

Vynorius, K. C., Paquin, A. M., \& Seichepine, D. R. (2016). Lifetime Multiple Mild Traumatic Brain Injuries Are Associated with Cognitive and Mood Symptoms in Young Healthy College Students. Front Neurol, 7, 188. doi:10.3389/fneur.2016.00188

Wallace, J., Covassin, T., \& Beidler, E. (2017). Sex Differences in High School Athletes' Knowledge of Sport-Related Concussion Symptoms and Reporting Behaviors. J Athl Train, 52(7), 682-688. doi:10.4085/1062-6050-52.3.06 
Wallace, J., Covassin, T., \& Lafevor, M. (2016). Use of the stepwise progression return-to-play protocol following concussion among practicing athletic trainers. Journal of Sport and Health Science. doi:10.1016/j.jshs.2016.11.002

Wallace, J., Covassin, T., Nogle, S., Gould, D., \& Kovan, J. (2017). Knowledge of Concussion and Reporting Behaviors in High School Athletes With or Without Access to an Athletic Trainer. J Athl Train, 52(3), 228-235. doi:10.4085/1062-6050-52.1.07

Warden, D. L., Bleiberg, J., Cameron, K. L., Ecklund, J., Walter, J., Sparling, M. B., Reeves, D., Reynolds, K. Y., \& Arciero, R. (2001). Persistent prolongation of simple reaction time in sports concussion. Neurology, 57(3), 524-526.

Wasserman, E. B., Kerr, Z. Y., Zuckerman, S. L., \& Covassin, T. (2016). Epidemiology of Sports-Related Concussions in National Collegiate Athletic Association Athletes From 2009-2010 to 2013-2014: Symptom Prevalence, Symptom Resolution Time, and Returnto-Play Time. The American Journal of Sports Medicine, 44(1), 226-233. doi:papers3://publication/doi/10.1177/0363546515610537

Wells, T. (2010). Health Status and Performance of United States Air Force Airmen Following Mild Traumatic Brain Injury. Retrieved from Wright-Patterson AFB, OH: http://www.dtic.mil/docs/citations/ADA555843

West Point. (2014). Information for New Cadets and Parents: Class of 2014 (pp. 30). West Point, NY.

Whalley, M. G., Rugg, M. D., Smith, A. P., Dolan, R. J., \& Brewin, C. R. (2009). Incidental retrieval of emotional contexts in post-traumatic stress disorder and depression: an fMRI study. Brain Cogn, 69(1), 98-107. doi:10.1016/j.bandc.2008.05.008

Wilcox, B. J., Beckwith, J. G., Greenwald, R. M., Chu, J. J., McAllister, T. W., Flashman, L. A., Maerlender, A. C., Duhaime, A.-C., \& Crisco, J. J. (2014). Head impact exposure in male and female collegiate ice hockey players. Journal of Biomechanics, 47(1), 109-114. doi:papers3://publication/doi/10.1016/j.jbiomech.2013.10.004

Wilcox, B. J., Beckwith, J. G., Greenwald, R. M., Raukar, N. P., Chu, J. J., McAllister, T. W., Flashman, L. A., Maerlender, A. C., Duhaime, A.-C., \& Crisco, J. J. (2015).

Biomechanics of head impacts associated with diagnosed concussion in female collegiate ice hockey players. Journal of Biomechanics, 48(10), 2201-2204.

doi:papers3://publication/doi/10.1016/j.jbiomech.2015.04.005

Wilcox, B. J., Machan, J. T., Beckwith, J. G., Greenwald, R. M., Burmeister, E., \& Crisco, J. J. (2014). Head-Impact Mechanisms in Men's and Women's Collegiate Ice Hockey. Journal of Athletic Training, 49(4), 514-520. doi:papers3://publication/doi/10.4085/1062-605049.3.19

Wilde, E. A., McCauley, S. R., Hunter, J. V., Bigler, E. D., Chu, Z., Wang, Z. J., Hanten, G. R., Troyanskaya, M., Yallampalli, R., Li, X., Chia, J., \& Levin, H. S. (2008). Diffusion tensor imaging of acute mild traumatic brain injury in adolescents. Neurology, 70(12), 948-955. doi:10.1212/01.wnl.0000305961.68029.54

Wilk, J. E., Thomas, J. L., McGurk, D. M., Riviere, L. A., Castro, C. A., \& Hoge, C. W. (2010). Mild traumatic brain injury (concussion) during combat: lack of association of blast mechanism with persistent postconcussive symptoms. J Head Trauma Rehabil, 25(1), 914. doi:10.1097/HTR.0b013e3181bd090f

Wilkerson, G. (2012). Neurocognitive Reaction Time Predicts Lower Extremity Sprains and Strains. International Journal of Athletic Therapy \& Training, 17(6), 4-9. 
Williams, R. M., Puetz, T. W., Giza, C. C., \& Broglio, S. P. (2015). Concussion Recovery Time Among High School and Collegiate Athletes: A Systematic Review and Meta-Analysis. Sports Medicine, 45(6), 1-11. doi:papers3://publication/doi/10.1007/s40279-015-0325-8

Wilson, M. R., Wood, G., \& Vine, S. J. (2009). Anxiety, attentional control, and performance impairment in penalty kicks. J Sport Exerc Psychol, 31(6), 761-775.

Witchalls, J., Blanch, P., Waddington, G., \& Adams, R. (2012). Intrinsic functional deficits associated with increased risk of ankle injuries: a systematic review with meta-analysis. Br J Sports Med, 46(7), 515-523. doi:10.1136/bjsports-2011-090137

Wunderle, K., Hoeger, K. M., Wasserman, E., \& Bazarian, J. J. (2014). Menstrual phase as predictor of outcome after mild traumatic brain injury in women. J Head Trauma Rehabil, 29(5), E1-8. doi:10.1097/HTR.0000000000000006

Xiong, Y., Gu, Q., Peterson, P. L., Muizelaar, J. P., \& Lee, C. P. (1997). Mitochondrial dysfunction and calcium perturbation induced by traumatic brain injury. J Neurotrauma, 14(1), 23-34. doi:10.1089/neu.1997.14.23

Yogev-Seligmann, G., Hausdorff, J. M., \& Giladi, N. (2008). The role of executive function and attention in gait. Mov Disord, 23(3), 329-342; quiz 472. doi:10.1002/mds.21720

Yoshino, A., Hovda, D. A., Kawamata, T., Katayama, Y., \& Becker, D. P. (1991). Dynamic changes in local cerebral glucose utilization following cerebral conclusion in rats: evidence of a hyper- and subsequent hypometabolic state. Brain Res, 561(1), 106-119.

Zhang, J., Yoganandan, N., Pintar, F. A., \& Gennarelli, T. A. (2006). Role of translational and rotational accelerations on brain strain in lateral head impact. Biomedical sciences instrumentation, 42, 501-506.

Zhang, L., Yang, K. H., \& King, A. I. (2004). A proposed injury threshold for mild traumatic brain injury. Journal of Biomechanical Engineering, 126(2), 226-236. doi:papers3://publication/doi/10.1115/1.1691446

Zhao, W., Ruan, S., \& Ji, S. (2015). Brain pressure responses in translational head impact: a dimensional analysis and a further computational study. Biomech Model Mechanobiol, 14(4), 753-766. doi:10.1007/s10237-014-0634-0

Zuckerman, S. L., Apple, R. P., Odom, M. J., Lee, Y. M., Solomon, G. S., \& Sills, A. K. (2014). Effect of sex on symptoms and return to baseline in sport-related concussion. $J$ Neurosurg Pediatr, 13(1), 72-81. doi:10.3171/2013.9.PEDS13257

Zuckerman, S. L., Kerr, Z. Y., Yengo-Kahn, A., Wasserman, E., Covassin, T., \& Solomon, G. S. (2015). Epidemiology of Sports-Related Concussion in NCAA Athletes From 2009-2010 to 2013-2014: Incidence, Recurrence, and Mechanisms. The American Journal of Sports Medicine, 43(11), 2654-2662. doi:papers3://publication/doi/10.1177/0363546515599634

Zuckerman, S. L., Lee, Y. M., Odom, M. J., Solomon, G. S., Forbes, J. A., \& Sills, A. K. (2012). Recovery from sports-related concussion: Days to return to neurocognitive baseline in adolescents versus young adults. Surg Neurol Int, 3, 130. doi:10.4103/2152-7806.102945 